Single-molecule measurements of Kinesin motor proteins

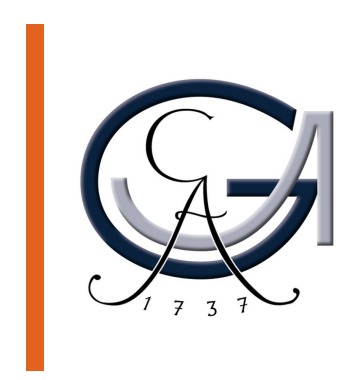

by André Düselder 



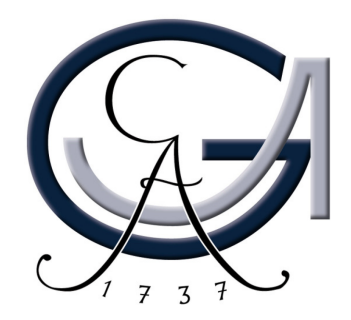

\title{
Single-molecule measurements of Kinesin motor proteins
}

\author{
Dissertation \\ for the award of the degree \\ „Doctor rerum naturalium" \\ of the Georg-August-Universität Göttingen
}

within the doctoral program

Physics of Biological and Complex Systems

of the Göttingen Graduate School for Neurosciences, Biophysics, and

Molecular Biosciences (GGNB)

of the Georg-August University School of Science (GAUSS)

submitted by

André Düselder

from Leer, Germany

Göttingen, October $30^{\text {th }}, 2013$ 
Members of the thesis committee:

Prof. Dr. Christoph F. Schmidt ( $1^{\text {st }}$ reviewer and supervisor) Drittes Physikalische Institut

Georg-August Universität Göttingen

Prof. Dr. Sarah Köster ( $2^{\text {nd }}$ reviewer)

Institut für Röntgenphysik

Georg-August Universität Göttingen

Dr. Oskar Hallatschek

Abteilung für Biologische Physik und Evolutionäre Dynamik

Max-Planck-Institut für Dynamik und Selbstorganisation, Göttingen

Additional members of the examination committee:

Prof. Dr. Jörg Großhans

Zentrum Biochemie und Molekulare Zellbiologie

Universitätsmedizin Göttingen

Dr. Iwan Schaap

Drittes Physikalische Institut

Georg-August Universität Göttingen

Dr. Claus Heussinger

Institut für Theoretische Physik

Georg-August Universität Göttingen

Date of the oral examination: December $11^{\text {th }}, 2013$ 


\section{Affidavit}

I herewith declare that I have produced this dissertation without the prohibited assistance of third parties and without making use of aids other than those specified; notions taken over directly or indirectly from other sources have been identified as such. This dissertation has not previously been presented in identical or similar form to any other German or foreign examination board.

The thesis work was conducted from November 2009 to October 2013 under the supervision of Prof. Dr. Christoph F. Schmidt at the Third Institute of Physics Biophysics. 



\section{Preface}

In November 2009, I started my PhD under the supervision of Prof. Christoph F. Schmidt in the Third Institute of Physics - Biophysics of the Georg-August University of Göttingen. The aim of my project was to study mitotic Kinesin-5 motor proteins, preferably in a way that resembles the conditions of the mitotic spindle as closely as possible. Along the path to reach this goal, many opportunities arose to look at other Kinesin related projects. Eventually, the scope of this thesis got broader and in the end, not only the study of mitotic Kinesin- 5 motors in a native environment, but also the more general investigation of regulatory processes in Kinesin became a central part.

This thesis covers the developments and new findings on different regulatory processes in Kinesin-5 motor proteins. The first two chapters give a general introduction into motor proteins and some of the well established experiments to study them. In Chapter 1, molecular motors, especially the subfamily of Kinesin-5 proteins, their properties and importance for cellular life, are introduced. The theoretical as well as experimental aspects of optical trapping are covered in Chapter 2. In addition, experimental methods and Kinesin proteins that are used throughout this thesis are examined.

The first results concerning the mechanisms of Kinesin regulation are presented in Chapter 3. Here, a hypothesis is studied that tries to explain how processive motion in molecular motors is possible. The assumption was that intra-molecular communication is facilitated by the transmission of tension by the neck linker of a Kinesin protein. This chapter is based on the publication:

André Düselder*, Christina Thiede*, Christoph F. Schmidt and Stefan Lakämper; 2012: Neck-Linker Length Dependence of Processive Kinesin-5 Motility, Journal of Molecular Biology, 423: 159-168. *Authors contributed equally.

which I conducted in collaboration with Christina Thiede. We studied a set of Kinesin constructs with different length of the neck linker which we expected to influence the motors' properties. Christina did most of the single-molecule fluorescence assays, while I focused on the optical-trapping experiments. We were able to show that the simple model is, at least for our constructs, not true. At the same time, we found evidence that supports a different explanation and might indicate a conserved mechanism in all Kinesins. 
Another study, that was performed in collaboration with the group of Leah Gheber of the Ben-Gurion University of the Negev, Beer-Sheva, Israel, is described in Chapter 4. In recent studies, the ability of Kinesin-5 motors from Saccharomyces cerevisiae to switch their direction of movement was reported. This is contradicting the previous observation that processive Kinesin motors exclusively move to the plus end of microtubules. The mechanism, by which this is achieved, is completely unknown. We hypothesize that the interaction of the tail domains of a Kinesin- 5 with the adjacent head groups might be able to regulate the directionality. To test this, different constructs of the $S$. cerevisiae motors without tail domains were designed. I suggested building a chimera that only retains the motility generating sub-units, i.e. the motor domains and neck linker, of the wild type motor. I performed extensive optical-trap as well as single-molecule fluorescence studies with this construct. The results will be published in a paper that is currently in preparation:

André Düselder, Christina Thiede, Alice Wiesbaum, Vladimir Fridman, Dieter Klopfenstein, Larisa Gheber and Christoph F. Schmidt: Directionality of Single Kinesin-5 Cin8 Molecules is Mediated by the Tail Domain.

Finally, Chapter 5 is dedicated to the original aim of my PhD project, the design and application of a new assay that mimics the environment of the mitotic spindle. So far, the motility of mitotic motors was most of the time investigated by using single-molecule techniques that either were in a load free environment, like singlemolecule fluorescence assays, or in a completely artificial environment, as in singlebead motility assays. To generate a situation that is as closely related to the mitotic spindle as possible, I used an optical trap to attach two small beads to a microtubule. This microtubule dumb-bell was positioned on top of another, surface-immobilized microtubule. Due to the size difference of microtubule and beads, this was only possible when the surface-bound filament was elevated. To achieve this, I developed a protocol to pattern a cover slip with micro pillars consisting of photoresist. With this technique, I was able to measure the native Kinesin-5 Eg5 as well as a tetrameric Kinesin-1/Kinesin-5 chimera in a loaded environment. Not only could I show that small angles between the filaments do not influence the native protein but I was also able to observe previously unknown characteristics of tetrameric motors that are only visible in this new assay.

The last chapter of this thesis, Chapter 6, summarizes the results of this work and tries to offer some points for future studies to begin.

This thesis covers examples of the regulatory processes that enable Kinesin motors to exhibit a vast range of different properties, even though they are structurally similar. The results, presented in the Chapters 3 to Chapter 5, provide some additional puzzle pieces that hopefully will help to complete our understanding of Kinesin- 5 motility in particular and molecular motors in general. 




\section{Table of contents}

Glossary $\quad$ xi

Chapter 1 Introduction $\quad 1$

1.1. Kinesin molecular motors: Walking, gating and switching . . . 2

1.2. Mitotic Kinesin-5 motor proteins . . . . . . . . . . . 7

$\begin{array}{lll}\text { Chapter } 2 & \text { Materials and methods } & 13\end{array}$

2.1. Optical trapping . . . . . . . . . . . . . . . . . . . . . . 13

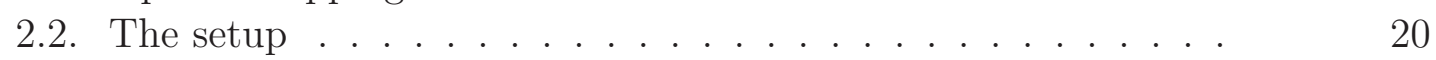

2.3. Experimental approaches . . . . . . . . . . . . . . . . . . . 22

2.4. Protein expression and purification . . . . . . . . . 28

Chapter 3 Neck-linker length dependence of processive Kinesin-5 motility 31

3.1. Influence of neck-linker length on velocity . . . . . . . . . . 34

3.2. Force generation is independent of neck-linker length . . . . . 37

3.3. Step size is unchanged by variation of neck-linker length . . . 39

3.4. Varying neck-linker length affects motor run length . . . . . . 41

3.5. Summary ... . . . . . . . . . . . . . . . 43

Chapter 4 Directionality of single Kinesin-5 Cin8 molecules is mediated by the tail domains $\quad 45$

4.1. Single molecules of Cin8Kin move bidirectional . . . . . . . . . . . . 46

4.2. Force generation of Cin8Kin molecules . . . . . . . . . . . . . . 49

4.3. Cin8 tail domains regulate directionality . . . . . . . . . 55

4.4. Cin8 tail domains are required for microtubule crosslinking . . 57

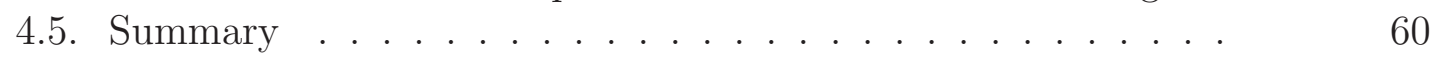

Chapter 5 In vitro studies of mitotic Kinesin motors in their native environment $\quad 63$

5.1. The suspended-microtubule assay . . . . . . . . . . 65

5.2. Chimeric Kinesin-1/Kinesin-5 microtubule-sliding motor DK4mer 70

5.3. Xenopus laevis mitotic Kinesin-5 Eg5 . . . . . . . . . . . . . 75

5.4. Saccharomyces cerevisiae mitotic Kinesin-5 Cin8 . . . . . . . . 81

5.5. Summary ............................ 82

$\begin{array}{lll}\text { Chapter } 6 & \text { Conclusions } & 87\end{array}$ 
$\begin{array}{ll}\text { A Biochemical protocols } & \mathbf{9 5}\end{array}$

A.1. Coating of beads . . . . . . . . . . . . . . 95

A.2. Preparation of microtubules . . . . . . . . . . . . . 97

A.3. Preparation of cover slips . . . . . . . . . . . . . . . . . . 99

A.4. Isolation and purification of axonemes . . . . . . . . . . 100

$\begin{array}{ll}\text { List of references } & 105\end{array}$

$\begin{array}{ll}\text { Acknowledgements } & 115\end{array}$

$\begin{array}{ll}\text { Curriculum Vitae } & 117\end{array}$ 


\section{Glossary}

$\begin{array}{ll}\text { ADP } & \text { Adenosine-5'-diphosphate } \\ \text { AMP-PNP } & \text { Adenosine-5'-( } \beta, \gamma \text {-imido)triphosphate } \\ \text { ATP } & \text { Adenosine-5'-triphosphate } \\ \text { BFP } & \text { Back-focal-plane } \\ \text { BRB80 } & \text { 80 mM PIPES buffer } \\ \text { DETA } & \text { 3-(2-[2-aminoethylamino]ethylamino) propyl-trimethoxysilane } \\ \text { DmKHC } & \text { Drosophila melanogaster } \text { Kinesin-1 heavy chain } \\ \text { DMSO } & \text { Dimethyl sulfoxide } \\ \text { DTT } & \text { Dithiothreitol } \\ \text { EDC } & \text { N-(3-Dimethylaminopropyl)-N'-ethylcarbodiimide hydrochloride } \\ \text { EDTA } & \text { Ethylene diamine tetraacetic acid } \\ \text { EGTA } & \text { Ethylene glycol tetraacetic acid } \\ \text { GFP } & \text { Green fluorescent protein } \\ \text { GMP-CPP } & \text { Guanosine-5'-[( } \alpha, \beta) \text {-methyleno]triphosphate } \\ \text { GTP } & \text { Guanosine-5'-triphosphate } \\ \text { NA } & \text { Numerical aperture } \\ \text { NL } & \text { Neck linker } \\ \text { MSD } & \text { Mean square displacement } \\ \text { NEM } & \text { N-ethylmaleimide } \\ \text { PIPES } & \text { Piperazine-N,N'-bis(2-ethanesulfonic acid) } \\ \text { PGMEA } & \text { Propylene glycol monomethyl ether acetate } \\ \text { PSD } & \text { Power spectral density } \\ \text { SBMA } & \text { Single-bead motility assay } \\ \text { SD } & \text { Standard deviation } \\ \text { SEM } & \text { Standard error of the mean } \\ \text { Sf9 } & \text { Spodoptera frugiperda } \\ \text { SGA } & \text { Surface-gliding assay } \\ \text { SMFA } & \text { Single-molecule fluorescence assay } \\ \text { SPB } & \text { Spindle-pole body } \\ \text { TIRF } & \text { Total internal reflection fluorescence } \\ \text { TMR } & \text { Tetramethylrhodamine } \\ \text { Tris } & \text { Tris(hydroxymethyl)-aminomethan } \\ \text { ATH } & \end{array}$





\title{
\begin{tabular}{l|l} 
& \\
Introduction & 1
\end{tabular}
}

\author{
"(...) equilibrium is death." \\ Henry Adams in Letter to American Teachers of History (1910).
}

In the year 1854, Rudolf Clausius gave the first mathematical definition of what he later called the entropy, S, of a system ${ }^{1}$. Entropy is the amount of functional energy that is dissipated in an (irreversible) process and thus cannot be transformed into useful work. This led to the formulation of the second law of thermodynamics

$$
d S \geq 0
$$

which states that the entropy of an isolated system will increase until the system reaches it thermodynamic equilibrium. Or, in other words, every system strives for a state of maximum disorder.

In 1944, Erwin Schrödinger noted in his book What is Life? [1] that living systems, at first glance, seem to defy the second law of thermodynamics. They display marvelous levels of order created from disorder. Schrödinger solved this dilemma by turning to nonequilibrium thermodynamics. He realized that living systems exist in a world of energy and material fluxes, i.e. they are open systems and not isolated, as the application of the second law demands. An organism stays alive in its highly organized state by taking energy from outside itself, from a larger encompassing system, and processing it to produce a lower entropy, more organized state within itself. If we transform any organism into an isolated system, Clausius' principle will soon get the upper hand, proving the above quotation true. Schrödinger recognized that life is a far-from-equilibrium state that maintains its local level of organization at the expense of the larger global entropy budget.

The same is true for cellular organisms or just single cells. If we, for example, look at single (living) cells we find that, in the low frequency regime, the response of the cell to outside stimuli deviates drastically from the response calculated from its spontaneous displacement fluctuations [2]. This violation of the fluctuationdissipation theorem, which connects the thermal fluctuations of a given system with its dissipation of energy [3], is a direct result of non-equilibrium processes inside the cell. A large variety of these non-equilibrium, i.e. actively driven, processes exist inside a single cell.

${ }^{1}$ Published in Poggendoff's Annalen, December 1854, vol. xciii. p. 481; translated in the Journal de Mathematiques, vol. xx. Paris, 1855, and in the Philosophical Magazine, August 1856, s. 4. vol. xii, p. 81 
Some of these processes involve elements of the cytoskeleton and associated molecular machines. These machines, or motor proteins, use the hydrolysis of Adenosine-5'-triphosphate (ATP) molecules as an outside energy source to conduct active work. Thus, motor proteins are able to form and maintain complex protein structures and generate concentration gradients by actively transporting material. They are, in essence, creating order from disorder. Due to the diverse and crucial roles of molecular motors in the cell, their molecular functions and regulations are still the focus of on-going research.

Three major families of cytoskeletal motor proteins are distinguished: the actinassociated Myosin motors; the minus-end directed, microtubule-associated Dynein motors and the microtubule-associated Kinesin motor proteins which are the subject of this thesis.

More than three decades ago, the first Kinesin was discovered [4]. Since that time, approximately 150 members of the Kinesin family have been identified and categorized into 14 subfamilies [5]. They are involved in a multitude of cellular processes, ranging from the transport of organelles and vesicles to the generation of cellular forces and locomotion as well as cell division.

Kinesins move along a microtubule by using energy from the hydrolysis of ATP molecules to generate conformational changes. The hydrolysis of each ATP molecule is tightly coupled to one step along the microtubule by a complex chemo-mechanical cycle (Figure 1.3 on page 6) [6]. The size of each step is $\sim 8 \mathrm{~nm}$, which directly corresponds to the distance between two motor binding sides on the microtubule lattice $^{2}$ [7]. While sharing a great similarity in the sequence of their motor domain (35-45\%), the part of the protein that contains the microtubule-binding site and the ATP-hydrolysis domain [8], the velocity of Kinesin motors varies largely between the different subfamilies. It ranges from $30-50 \mathrm{~nm} / \mathrm{s}$ for Kinesin-5 [9, 10] to more than $1500 \mathrm{~nm} / \mathrm{s}$ for Kinesin-1 [11, 12]. Single Kinesin motors can typically generate forces in the range of a few piconewton, again depending on the subfamily (less than $2 \mathrm{pN}$ for Kinesin-5 [10], up to $7 \mathrm{pN}$ for Kinesin-1 [6]). To be able to produce this vast range of motile properties throughout the subfamilies, some underlying mechanisms and regulatory processes must exist. The aim of this thesis is to identify these regulations and to gain some understanding of how they affect Kinesin motor proteins.

\section{\begin{tabular}{l|l} 
& Kinesin motors: \\
1.1 & Walking, gating and switching
\end{tabular}}

Kinesin motors have been studied for nearly four decades. During this time, a whole variety of single-molecule experiments have been performed with the aim of discovering how these remarkable protein machines function. Still, the details of a single Kinesin step are not completely understood. To introduce the properties of Kinesin motor proteins a selection of questions is posed in the following. The

\footnotetext{
${ }^{2}$ Motor binding sites are contained in $\beta$-tubulin monomers. Alternating subunits of $\alpha$ - and $\beta$ tubulin monomers form one long protofilament. 13 protofilaments laterally arranged form a single hollow, cylindrical microtubule.
} 
answers to these questions have either led to impressive progress in understanding Kinesin motility or are still the subject of on-going research.

\section{What is the walking pattern of Kinesin?}

The walking pattern of Kinesin has caused many discussions. Hua et al. introduced three different models for the Kinesin walk: (i) the symmetric hand-over-hand model where the two heads exchange leading and trailing positions on the microtubule, but the three-dimensional structure of the Kinesin molecule is preserved at all equivalent points in the stepping cycle (Figure $1.1 \mathrm{~A}$ ), (ii) the asymmetric hand-over-hand model where the Kinesin heads exchange positions on the microtubule, but the initial and final states of the molecule are not symmetry-related (Figure 1.1 B), and (iii) the inchworm model where one head always leads and the other always trails during the cycle of advancement (Figure 1.1 C) [13].

In the same study, Hua et al. ruled out the symmetric hand-over-hand $(\mathrm{HoH})$ model. Microtubules, attached to the C-terminus of the protein, did not show large angular displacements during Kinesin stepping. The symmetric $\mathrm{HoH}$ model predicts a rotation of the stalk during one step by $180^{\circ}$ and is therefore inconsistent with these findings [13].

The subsequent observation of "limping" Kinesins strongly favored the asymmetric HoH model. Different Kinesin constructs, both hetero- as well as homodimeric molecules, were found to alternate between two distinct different states with each step $[14,15]$. Different states in a homodimeric protein, i.e. different dwell times before the next step is taken, suggest that even though the structural elements of each step are the same, the actual trajectories might be different. Two different steps, "left" and "right", agree with the expectations from the asymmetric HoH model.

Moreover, Gutiérrez-Medina et al. showed that while the stalk of dimeric Kinesin did not rotate with every step [13], in $\sim 2 \%$ of the steps random $\pm 180^{\circ}$ reorientations were evident [16]. They concluded that stepping imperfections lead, on occasion, to two left or right steps executed in a row, which requires a reorientation of the stalk. This further supports the notion of two different steps (Figure 1.1 B).

The inchworm model was ruled out in a compelling experiment in 2004. Yildiz et al. followed the motion of an individual head of a dimeric Kinesin, labeled by a single fluorophore. They used video centroid tracking with an accuracy of $\sim 1 \mathrm{~nm}$ to show that the labeled head takes $16 \mathrm{~nm}$ steps [17]. This is contradicting the inchworm model in which every individual head only takes $8 \mathrm{~nm}$ steps.

With great certainty, we can concluded from the combination of these different experiments that Kinesins walk in an asymmetric hand-over-hand fashion.

Another key question was whether a Kinesin motor moves along a single protofilament $[18,19]$ or astride two adjacent protofilaments [20, 21]. The work of Fehr et al. on limping Kinesins not only showed that the step size of the "left" and "right" step was identical but also that an increase in longitudinal load on the motor almost exclusively affected one of the two steps $[22,23]$. They further argued that this indicates that the motor alternately steps on two adjacent protofilaments.

In contrast, a compelling study performed by Schaap et al. with atomic force mi- 


\section{A Symmetric hand-over-hand model}
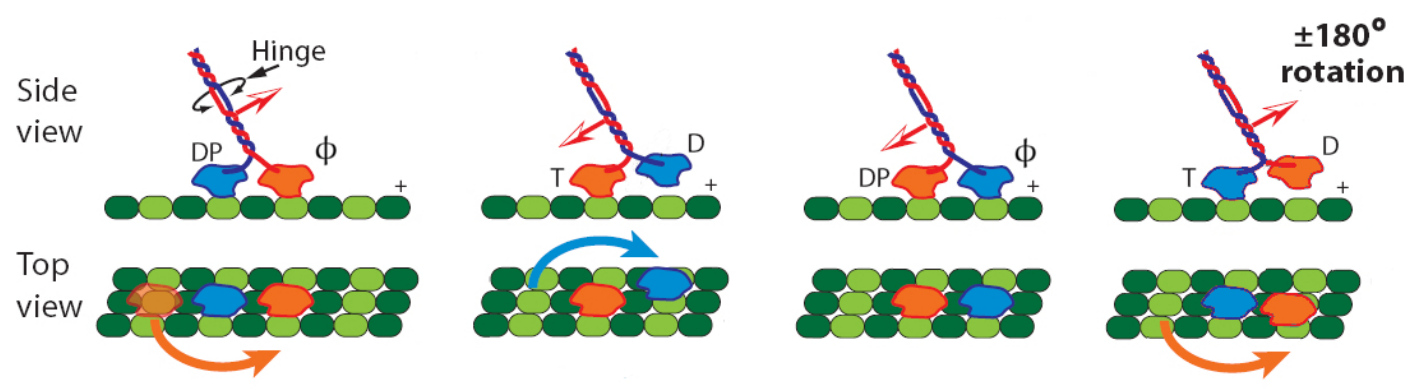

\section{B Asymmetric hand-over-hand model}
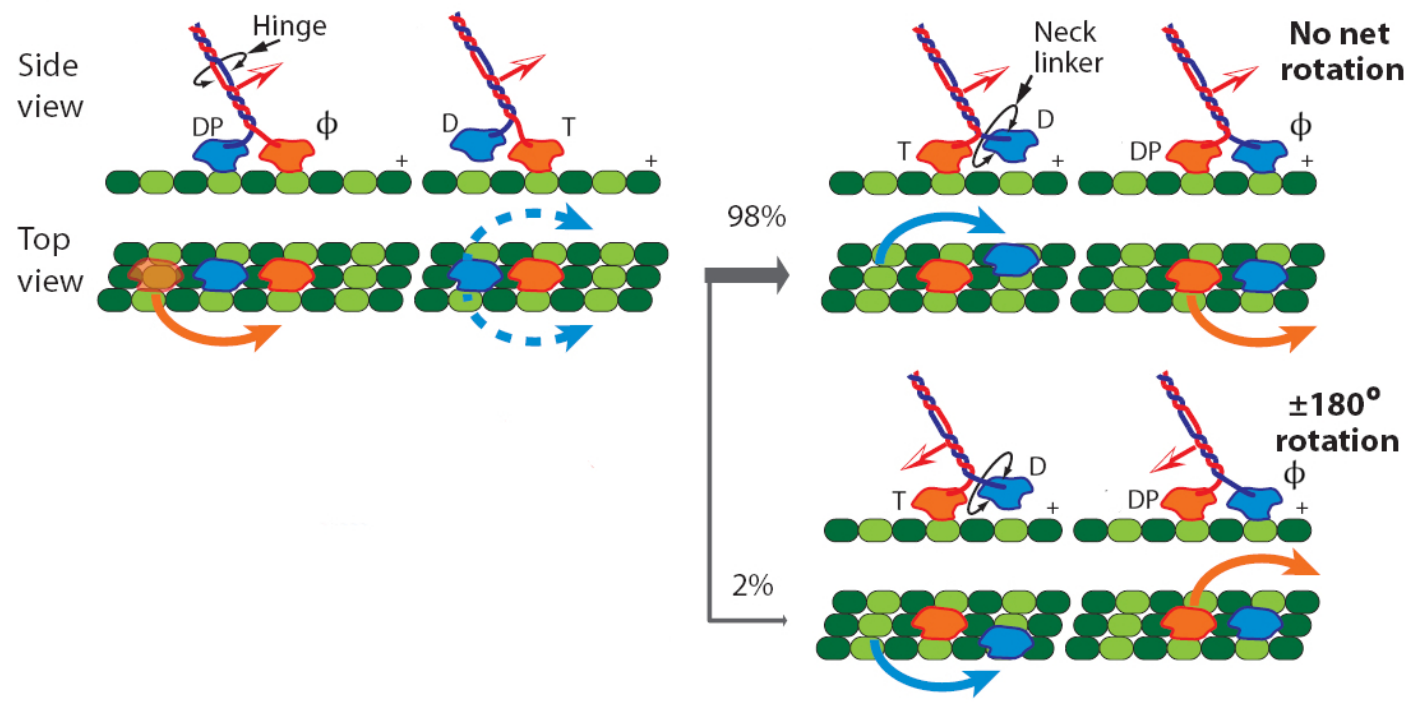

\section{Inchworm model}

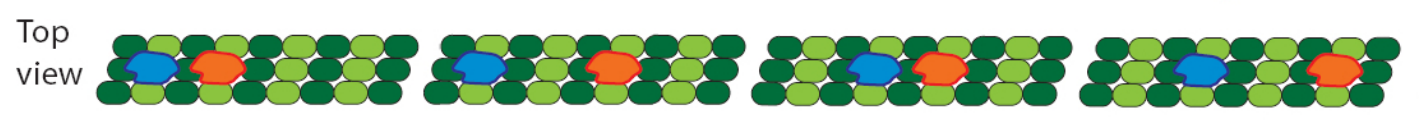

Figure 1.1: Kinesin stepping models

A In the symmetric hand-over-hand model, the details of every step are identical. To revert to the same physical state, the motor has to rotate its stalk (orientation indicated by red and white arrow) rotates $180^{\circ}$ clockwise and counterclockwise every other step for symmetric walking. B During asymmetric hand-over-hand stepping, the stalk does not rotate. To distinct steps with different molecular details exist. When taking a regular step, the blue head passes its partner on the left. However, in $\sim 2 \%$ of the steps, the blue head passes on the right instead of the left, generating two right steps in a row. The orientation of the stalk is now reversed. C In the inchworm model the two heads retain their nonequivalent positions at the beginning of each successive cycle. The front head always leads while the rear head stays the trailing head at all times. The motor reverts to the same conformation without requiring the stalk to rotate. Figure adapted from [16].

The different nucleotide binding states are termed as follows: T - ATP bound, DP - ADP and phosphate bound, D - ADP bound, $\phi$ - no nucleotide bound. 
Figure 1.2: Single Kinesin motors moving along a microtubule (A-D) Four frames (30 s acquisition time per frame) of a movie obtained with an AFM. A Two Kinesin dimers, indicated by arrows. B Both motors have proceeded (of the leading dimer only the last head is still visible). C The second $\mathrm{Ki}$ nesin has proceeded further. D The second Kinesin has also disappeared. (E and $\mathbf{F}$ ) Two frames from another sample show a displacement of $16 \mathrm{~nm}$. The two Kinesin heads are clearly visible. Fiduciary marks on the background were used to correct for drift. The position of the arrow is fixed in both frames with respect to the fiduciary marks. Figure adapted from [24].
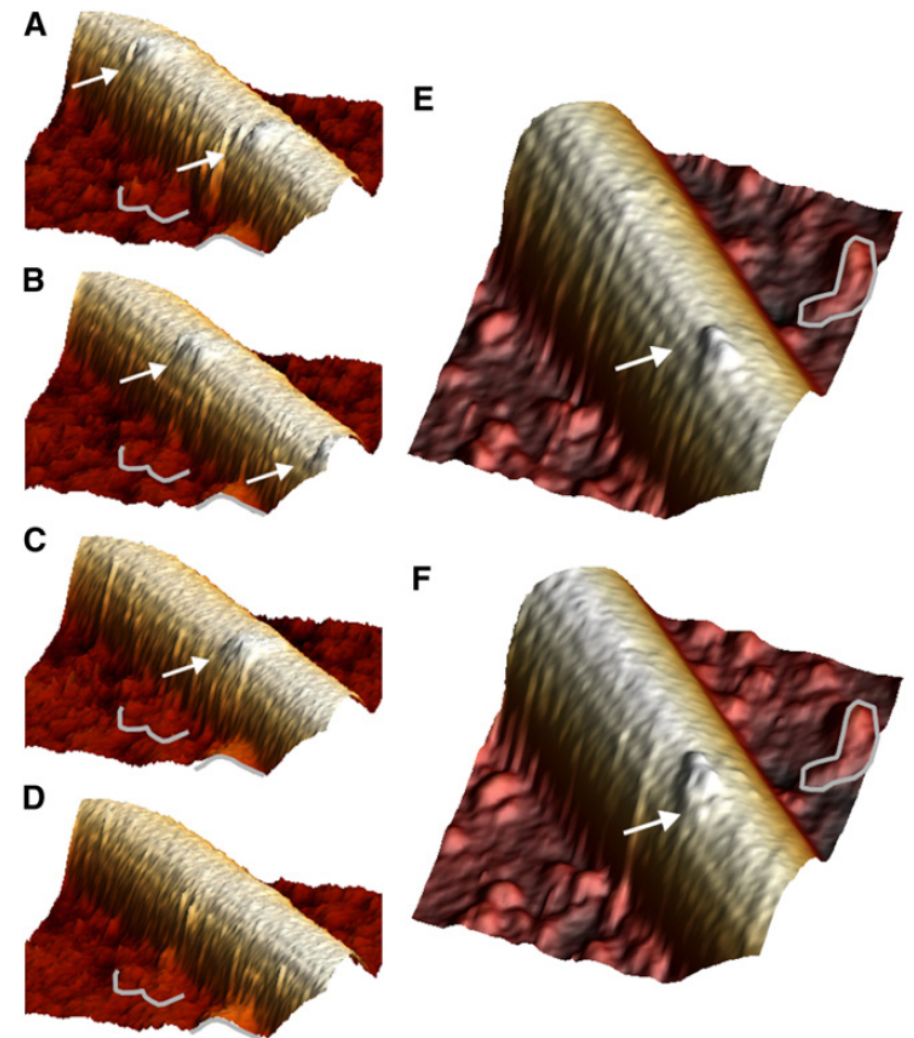

croscopy indicated that Kinesin-1 moves differently. They imaged single Kinesin-1 motors at submicromolar ATP concentrations with nanometer resolution and showed that the heads of an individual Kinesin are bound to the same protofilament (Figure 1.2) [24]. This strongly suggests that Kinesin motors actually walk along single protofilaments.

\section{How can Kinesin move processively?}

Kinesins, with the exception of members of the Kinesin-14 family ${ }^{3}$, are capable of processive motion. They are able to walk along a single microtubule for up to several micrometers, taking hundreds of successive $8 \mathrm{~nm}$ steps [28, 29]. For a single motor to be capable of processive motion, at least one of the two motor heads is required to be in contact with the microtubule at all times. Processive Kinesins are therefore likely to use similar mechanisms to keep the motor heads out of phase during the chemo-mechanical stepping cycle [30, 31, 32].

One possible mechanism is believed to be head-head communication mediated by intra-molecular tension $[34,35,36]$. The state in the stepping cycle, when both heads are bound to the microtubule, is the most mechanically tensed (state (1) in Figure 1.3). X-ray structures of dimers in this state can only be brought into accordance with cryo-electron microscopy images if the structural elements that link the two head domains, the neck linker, are in a stretched conformation [37]. These neck linker, mostly unstructured amino acid chains connecting the motor domains to

\footnotetext{
${ }^{3}$ Kinesin-14 are motors with a C-terminal motor domain. They move in a non-processive fashion
} by producing isolated minus-end directed power strokes [25, 26, 27]. 


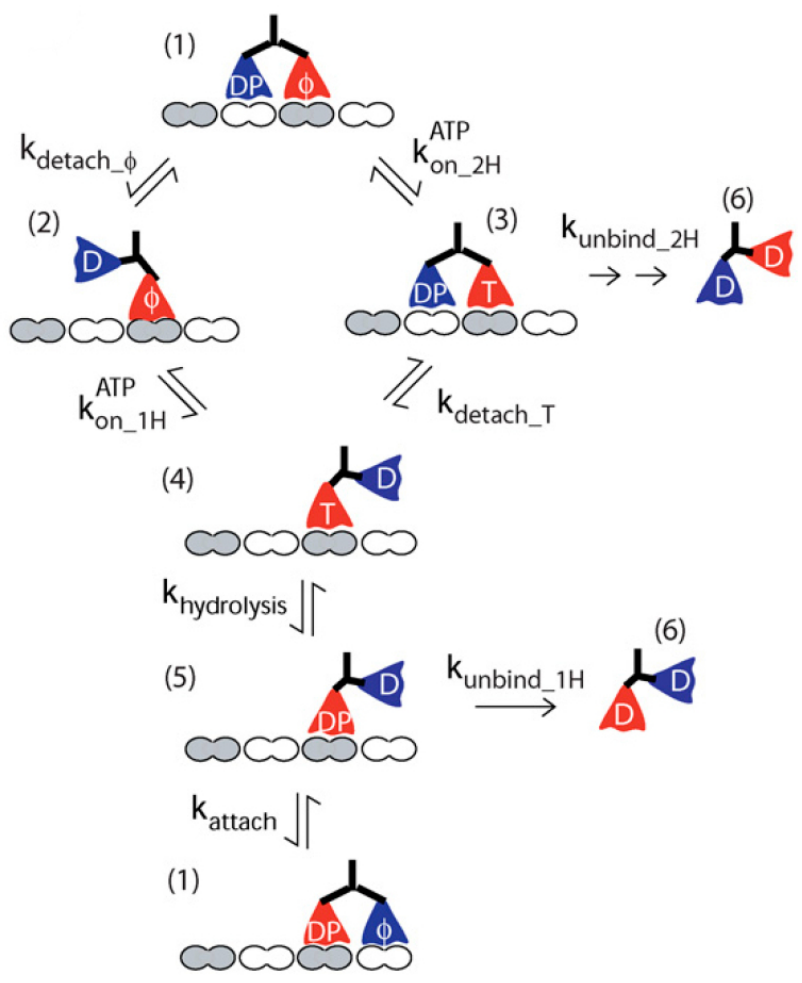

Figure 1.3: Model for the chemomechanical stepping cycle Possible model for the Kinesin ATP hydrolysis cycle. After one sequence $((1)$ - (2) - (4) - (5) $\rightarrow(1))$ the motor is again in its initial state. The step from (1) to (3) describes premature ATP binding to the leading head. This can lead to the unbinding of the motor (state (6), transition states omitted) and might be suppressed by front-head gating. The motor unbinding due to premature release of phosphate from the trailing head (transition from state (5) to (6)) could be prevented by rearhead gating (see text for more details). The different nucleotide binding states are termed as follows: T - ATP bound, DP - ADP and phosphate bound, D - ADP bound, $\phi$ - no nucleotide bound. Figure adapted from [33].

the stalk coiled coil $[38,39]$, were suggested to be the tension-transmitting elements. When modeled as a flexible polymer chain, the tension in the neck linker at a certain end-to-end distance would scale inversely with contour length [40]. From this observation, two possible gating mechanisms were postulated: (i) The front-head gating model assumes that the leading, nucleotide-free head is under backward tension and therefore unable to bind a new ATP molecule as long as the trailing head is bound to the microtubule $\left(\mathrm{k}_{\text {detach } \_\phi} \gg \mathrm{k}_{\mathrm{On} \_2 \mathrm{H}}^{\mathrm{ATP}}\right.$ in Figure 1.3) [35]. This locks the leading head in a tightly bound state and prevents premature detachment of the motor protein. (ii) The rear-head gating model postulates that the release of phosphate from the rear head, leading to detachment, is accelerated by the forward tension generated from the binding of the leading head to the microtubule $\left(k_{\text {attach }} \gg k_{\text {unbind_1H }}\right.$ in Figure 1.3) [35, 41, 42]. These models are not mutually exclusive and Kinesins might use both mechanisms to coordinate processive stepping [43].

A more recent study presented an alternative to this simple model. Instead of the length and tension of the neck linker, its sterical orientation might play a crucial role [43]. In Chapter 3 we show that for a Kinesin-5 head/Kinesin-1 stalk chimera, the modification of the length of the neck linker had only a minor effect on the general motile properties. While velocity and force were not influenced, we observed a distinct change in run length upon varying the neck-linker length. This further supports the theory that the orientation of the neck linker is the determining factor for inter-head-communication and not the intra-molecular strain.

\section{Is Kinesin an unidirectional motor?}

Since the discovery of the first Kinesin, it was considered a fact that all Kinesins move unidirectional. A microtubule is build up by two different monomers, $\alpha$ - and 
$\beta$-tubulin, with different enzymatic activities. The alternating arrangement of these monomers in the microtubule give rise to two distinguishable sides: a faster growing plus end and a slower growing minus end. So far it was believed that nearly all members of the Kinesin family move toward the plus end of the microtubule [44] (the exception being the non-processive Kinesin-14 proteins that move toward the minus end [25]).

Surprisingly, two independent groups reported that the Kinesin-5 Cin8 from Saccharomyces cerevisiae was able to switch its direction of motion. The switching occurs either depending on the number of motors operating at the same time [45], the ionic strength of the buffer medium or the binding geometry of the motor [46, 47]. In addition, it was recently reported that the second Kinesin-5 homolog in yeast, Kip-1, is also capable of bidirectional motion [48].

Not only challenge these findings the 20-year-old dogma that Kinesin homologs which carry their catalytic domains at the N-terminus are plus-end directed [44], but this also is the first time that bidirectional motion was observed in a Kinesin motor protein. The molecular details of the switch that changes the movement direction of Cin8 and Kip1 are completely unknown. The fact that individual motors are capable of bidirectional movement and that binding of a second microtubule seems to facilitate a switch in direction of movement indicates a regulatory process that involves the tail domains. This hypothesis is further discussed in Chapter 4.

\section{Mitotic Kinesin-5 motor proteins}

Of the 14 subfamilies of the Kinesin motor proteins, the Kinesin- 5 subfamily is of particular interest. Characteristic of all Kinesin-5 motor proteins is the unique homotetrameric, bipolar structure with two pairs of N-terminal motor domains positioned at the ends of the tetramer's long axis [5, 49]. The founding member of the Kinesin-5 family was found in a genetic screen for temperature-sensitive, lethal mitotic genes in the fungus Aspergillus nidulans [50]. One discovered mutant form of a protein blocked the mitosis of the fungal cells. The protein was therefore named BimC (blocked in mitosis) [51]. A similar screen carried out in fission yeast identified a related Kinesin, Cut7 [52]. Mutations in either BimC or Cut7 blocked the separation of the spindle pole bodies and thus prevented the successful completion of mitosis in the respective systems $[51,52]$. Further studies have identified similar mitotic motor proteins in many other systems, e.g. the aforementioned Eg5 in Xenopus laevis and Cin8 and Kip1 in Saccharomyces cerevisiae, KLP61F in Drosophila melanogaster and hsEg5 in human [49, 53, 54, 55]. This group of related Kinesins localizes to spindle microtubules as well as structures present at spindle poles and was eventually classified as the Kinesin- 5 family of motor proteins[5].

The bipolar structure of the Kinesin-5 motor proteins allows them to bind to two microtubules simultaneously and crosslink them. It also enables single proteins to displace antiparallel aligned microtubules relatively to each other, a behavior that has been directly observed in vitro $[9,46,56]$. In vivo, these motors are of exceptional importance for the process of cell division as Kinesin- 5 motors are directly involved 
A
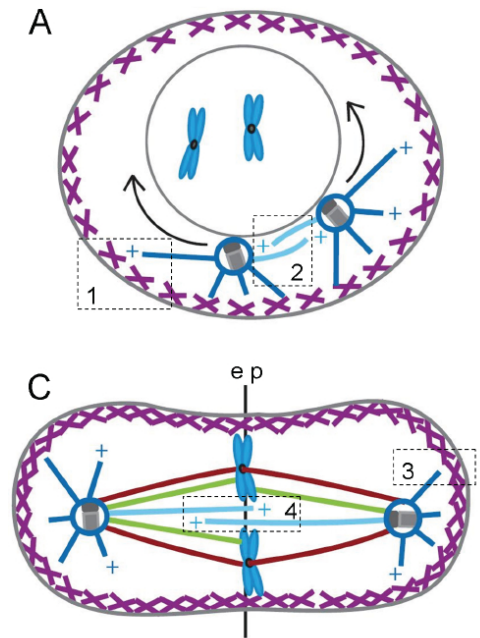

E

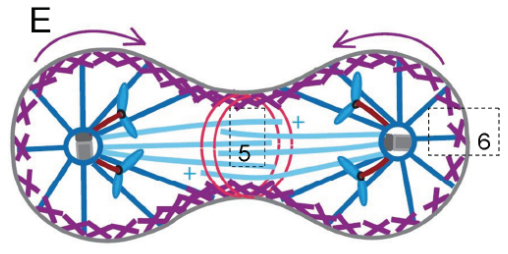

$x \times$ Cell cortex Centrosome 20

(48) Nucleus Centriole •
B

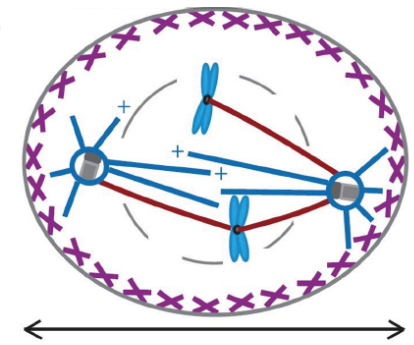

D

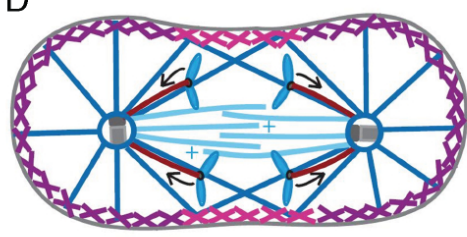

$\mathrm{F}$

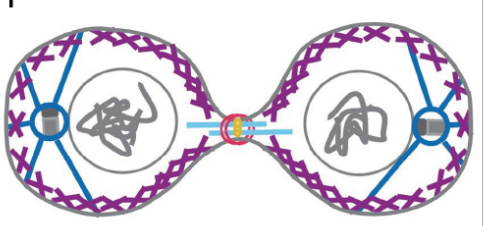

Chromosome $=$ Midbody

Kinetochore (i) Contractile ring

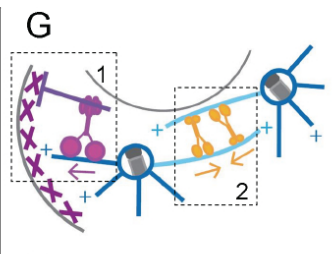

$\mathrm{H}$

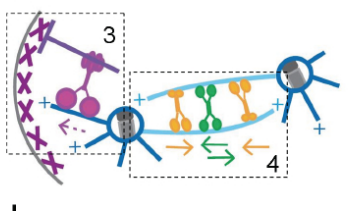

।

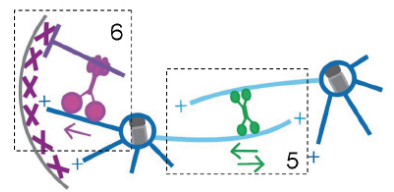

Astral MT

Kinetochore MT

Interpolar MT

Chromosomal MT

do Dimeric kinesin of Dynein

of Bipolar kinesin Dynactin

Figure 1.4: Overview of the processes during mitosis and cytokinesis

A Prophase, the replicated chromosomes condense and the duplicated centrosomes migrate around the nucleus, initiating the formation of the mitotic spindle. B Prometaphase, the breakdown of the nuclear envelope allows the kinetochore microtubules (red) to connect to the chromosomes and to move them toward the equatorial plane (ep). C Metaphase, the sister chromatids are aligned in the equatorial plane and via the kinetochore microtubules connected to the opposite spindle poles. D Anaphase $\mathrm{A}$, in the segregation process the chromatids are moved to opposite poles. Anaphase B, the spindle poles move apart due to the reorganization of the spindle microtubules. E During late anaphase the chromatids reach the spindle poles and the cleavage furrow containing the contractile ring assembles and begins to contract. $\mathbf{F}$ Telophase, the sister chromosomes decondense and new nuclear envelopes reassemble around them. Cytokinesis, the cytoplasm of one cell is divided in two by the contractile ring, which causes the furrow to ingress and finally seal, completing the separation of the two daughter cells. G - I Model of motor proteins in spindle morphogenesis. Not drawn to scale. Figure based on $[30,57]$, created by Christina Thiede. Also published in [59].

in the formation of the mitotic spindle (Figure 1.4) [30, 57, 58]:

During the onset of mitosis, the initial assembly of the mitotic spindle involves a dynamic balance of forces between the minus-end directed Dynein motors on the cortex (inset 1 in Figure 1.4) and the plus-end directed, dimeric Kinesins (inset 2 in Figure 1.4). Upon increase of the overlap zone of the interpolar microtubules, the inward force generated by Kinesin gradually increases until it balances the outward force generated by Dynein (Figure $1.4 \mathrm{G}$ ). In the beginning of metaphase, the astral microtubules have minimal length and so the outward force generated by Dynein is low (inset 3 in Figure 1.4). In the midzone (inset 4 in Figure 1.4), a balance between forces generated by bipolar, plus-end directed Kinesin-5 motors (green) as well as dimeric, plus-end directed Kinesins and interpolar microtubule bundles results in the movement of the chromosomes (Figure $1.4 \mathrm{H}$ ). When cortical Dynein together with the activity of Kinesin-5 overwhelms the inward forces generated by 

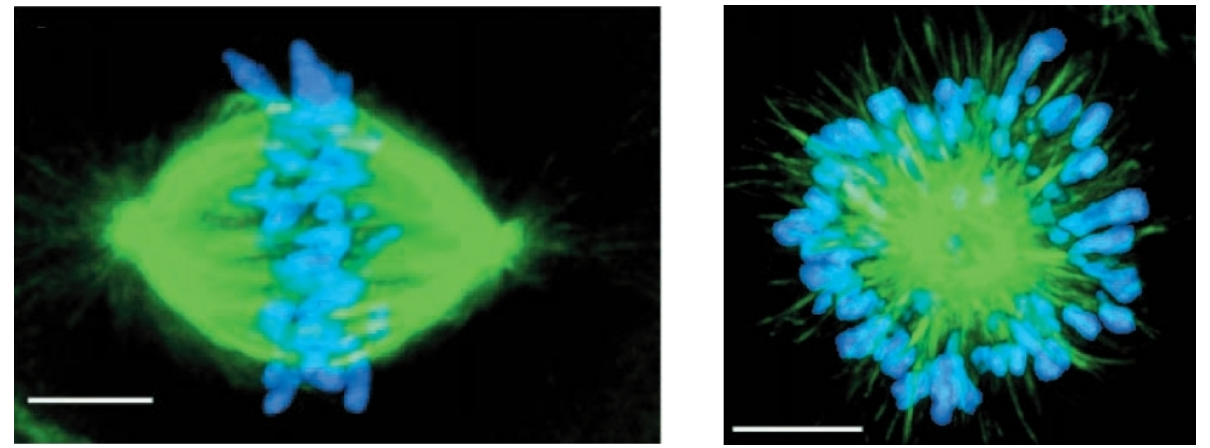

Figure 1.5: Inhibition of Kinesin-5 causes monoastral spindles

Monastrol, an Kinesin-5 inhibitor, causes the formation of monoastral spindles during mitosis in mammalian epithelial kidney cells. Immunofluorescence staining of $\alpha$-tubulin (green) and chromatin (blue). Treatment of mitotic cells with monastrol replaces the normal bipolar spindle (left) with a rosette-like microtubule array surrounded by chromosomes (right, incubated with $68 \mu \mathrm{M}$ monastrol). Scale bars: $5 \mu \mathrm{m}$. Figure adapted from [60].

the dimeric plus-end directed Kinesins, the balance within the spindle shifts towards the forces in the outward direction. This results in the elongated metaphase spindle. In anaphase B the sudden loss of the inward forces, caused by the inactivation of the dimeric Kinesins, leads to the release of the tension within the spindle (Figure $1.4 \mathrm{I})$. This causes a final elongation of the spindle.

The inhibition of Kinesin-5 motors in the mitotic spindle by certain substances (e.g. monastrol) leads to a disruption of the force balance during mitosis and results in a collapsed monoastral spindle (Figure 1.5). Cell division is no longer possible and apoptosis is triggered [60].

This illustrates the importance of Kinesin-5 molecular motors. These motors, their properties and especially the different regulatory processes affecting them are a major part of this work. In the following section, an overview about the present knowledge of several Kinesin-5 motors which are studied here will be given.

\section{Xenopus laevis Kinesin-5}

The mitotic Kinesin-5 Eg5 from Xenopus laevis moves toward the plus ends of microtubules. Its motility is highly complex: single Eg5 motor proteins show a mixture of diffusive and ATP-dependent directional motility along a single microtubule while staying attached for several tens of seconds [61, 62]. Interestingly, upon crosslinking of a second microtubule, diffusive motility is suppressed, and directional motility becomes dominant [63]. Previous studies have indicated that the tail domains of Kinesin motors regulate various aspects of their activity. For example, the tail domain of Kinesin-1 has been shown to inhibit its motility [64] by crosslinking the two catalytic domains with the tails in the active Kinesin dimer [65]. In addition, it has been recently shown that the tail domains of the Drosophila melanogaster kinesin-5 Klp61F, are located in the vicinity of - and are likely to interact with - the motor domains [66]. Since Eg5 contains a non-motor microtubule binding site in its taildomains [67], it seems plausible that the tail domains could register the presence or absence of a second microtubule and influence the motor domains. Thus the binding 
geometry, whether the motor is bound to one or two microtubules, might regulate the Eg5 motility.

As is evident from single-molecule fluorescence assays, Eg5 is a slow molecular motor with an average velocity of $\sim 20 \mathrm{~nm} / \mathrm{s}$. It is able to crosslink and slide apart antiparallel aligned microtubules. The motor will move on both antiparallel microtubules towards the plus end, i.e. in opposing directions. Hence, the relative velocity with which the two microtubules move away from each other is $\sim 40 \mathrm{~nm} / \mathrm{s}$, twice that of the motor on a single microtubule [9, 62]. Eg5s capabilities to generate forces, studied with single-bead motility assays, showed that Eg5 is able to build up forces that on average reach $1.6 \mathrm{pN}$ [10]. No stalling ${ }^{4}$ prior to unbinding could be detected [10]. So far, the motile properties, especially the generation of forces, of Eg 5 in its native geometry, the force balanced environment of the mitotic spindle, are mostly unknown. In Chapter 5 we show the first study of Eg 5 in this geometry, using micro fabricated structures and a dual-beam optical trap.

\section{Saccharomyces cerevisiae Kinesin-5}

Another member of the Kinesin-5 subfamily is Cin8 from Saccharomyces cerevisiae. Cin 8 was the first Kinesin found that is able to switch its direction of motion. In single-molecule fluorescence assays, individual Cin8 motors can be switched by ionic conditions from rapid $(\sim 400 \mathrm{~nm} / \mathrm{s})$ and processive minus-end to slow $(\sim 80 \mathrm{~nm} / \mathrm{s})$ plus-end motion on single microtubules $[46,47]$. Cin8 was also shown to switch from fast minus-end to slow plus-end directed motility when bound between antiparallel microtubules $[45,46]$. In this geometry, the two crosslinked microtubules were typically sliding apart with their minus ends leading with a relative velocity of about $30-60 \mathrm{~nm} / \mathrm{s}[46,47]$. As an Eg5 homolog, it is possible that the tail domains in Cin8 influence the protein. We therefore hypothesize that the tail domains of Cin8 are involved in the regulation of its directionality.

\section{Kinesin-5 head/Kinesin-1 stalk chimeras}

To study the intrinsic capabilities of Kinesin- 5 motor domains without any influence of the tail domains, the construction of chimeric Kinesin- 5 head/Kinesin- 1 stalk dimers is a promising technique. For our studies, we built chimeras consisting of the main force-generating parts of Eg5- or Cin8-motor domains and neck linker and fused these to the neck coiled coil of Drosophila melanogaster Kinesin-1 DmKHC. This approach presented us with stable dimeric chimeras that lacked the opposing part of the tetrameric structure and therefore the tail domains that are adjacent to the remaining heads. Since there is no evidence that the tail domains of one Kinesin influence the members of a different Kinesin subfamily, any effect of the Kinesin-1 tail on the Kinesin-5 motor domains is unlikely.

The chimeric construct, consisting of the motility-generating subunits of Eg5 fused to the DmKHC stalk, proved to be a useful model system for studying the

\footnotetext{
4"Stalling" describes the behavior of some molecular motors, in particular Kinesin-1, to stop moving but staying bound to a microtubule upon reaching a certain amount of opposing force.
} 
regulation of Eg5 motility. This motor, termed Eg5Kin, was a processive motor that moved unidirectional along single microtubules with an average velocity of $\sim 95 \mathrm{~nm} / \mathrm{s}$ and an average run length of $\sim 1.9 \mu \mathrm{m}$ [68]. In single-bead assays, it sustained forces of up to $\sim 5 \mathrm{pN}$ [68]. In a previous study, Eg5Kin did not show the diffusive motility of tetrameric wild type Eg5, adding to the evidence that the tail domains in $\mathrm{Eg} 5$ are mediating diffusive interactions.

In Chapter 3, we used a set of twelve Eg5Kin constructs with different length of the neck linker. With these motors, we examined the hypothesis whether tension, transmitted by the neck linker, is required for processive motion.

The Cin8 head/Kinesin-1 stalk chimera Cin8Kin was studied in Chapter 4 . In Cin8Kin, the loss of the tail domains changed the regulation of the motility of the motor while the bi-directionality of wild type Cin8 is conserved. The switching of direction of motion is highly regulated in Cin8 and only occurs in certain events. Cin8Kin, on the other hand, apparently chose its direction in every single step at random. Our hypothesis, that this loss of regulation is a direct consequence of the removal of the tail domains, is further strengthened by the analysis of a second construct. We removed the tail domains in wild type Cin8 to get the tetrameric Cin8 $\Delta$ tail construct where no head-tail interaction is possible.

\section{Kinesin-1 head/Kinesin-5 stalk chimera}

The inverse construct to the previously mentioned chimeras can be used to study mitotic Kinesin-5 motors as well. Since it is unlikely for Kinesin-5 tails to affect Kinesin- 1 heads, a Kinesin- 1 head/Kinesin- 5 stalk chimera allows to investigate Kinesin- 5 regulation. The properties of the stalk, including the tails, without any interaction between the tails and the motors heads can thus be analyzed.

In a previous study [69] the chimera DK4mer was characterized. DK4mer consists of the head domains of Drosophila melanogaster Kinesin-1 DmKHC fused to the stalk of Xenopus laevis Eg5, including the tail domains. The neck linker of Dk4mer is build up by the 14 amino acid long neck liner of DmKHC extended by the last four amino acids of the Eg5 neck linker to a total length of 18 amino acids.

Dk4mer is a fast tetrameric microtubule-sliding motor without any possible tail-head regulation, but with the additional tail binding sites located in the tail domains [66] as well as the bipolar structure of Eg5. In single-molecule fluorescence assays, it moved on single microtubules with a high average velocity of $\sim 500 \mathrm{~nm} / \mathrm{s}$ and a remarkably long run length of $\sim 9 \mu \mathrm{m}$ [69]. It was able to crosslink and slide apart two antiparallel aligned microtubules. Since DK4mer walked on both microtubules it crosslinks, the velocity of the moving microtubule was on average twice the velocity on single microtubules $(700-1100 \mathrm{~nm} / \mathrm{s})$ [69].

The molecular motors mentioned so far are only a small subset of the large number of different Kinesin-5 motors which are only a small part of the even larger family of Kinesin proteins. As the following chapters will demonstrate, the detailed study of the molecular mechanisms that govern the function of these proteins provides us with a deeper insight into not only the regulation of Kinesin-5 motors but also Kinesins in general. 



\section{Materials and methods}

To examine motor proteins and the mechanisms of their regulation, a whole range of different techniques are applied. In the following, the most common experiments, that are also used in the present work, are introduced. Especially the theoretical as well as practical aspects of optical trapping are covered. In addition, the cloning and purification of the motor proteins used throughout this thesis are described.

\section{Optical trapping $\quad 2.1$}

On a microscopic scale, life is a complex and chaotic interplay of biology, chemistry and physics. Brownian motion constantly jostles biomolecular components such as proteins, lipids, carbohydrates and nucleic acids. The active motion of biomolecules often happens on a level of several nanometers, well below the resolution limit of conventional light microscopy which is limited by optical diffraction. The weak forces that evolve around these biomolecules are typically in the piconewton range. To study the physics and chemistry of life, experimental methods are needed which are able to measure nanometer-length distances and piconewton-scale forces. Pioneered by Arthur Ashkin and co-workers at Bell labs nearly 25 years ago [70, 71, 72], the advent of the laser-based gradient-force optical trap, or "optical tweezers", provided an ideal tool to look at the biophysical details of the molecular world.

\section{Trapping theory $\quad 2.1 .1$}

Photons impinging on a dielectric particle will either be reflected or refracted. Both processes result in a change of momentum of the photons. The conservation of momentum dictates that this inevitably leads to a change of momentum of the dielectric particle, i.e. a force is induced on the particle. If the source of the photons is a collimated laser beam, it is easy to see that the generated radiation pressure will push the particle in the direction of the beam. The maximum force that a laser 
beam with power $P$ and wavelength $\lambda=c / \nu$ can exert on particles in a medium with refractive index $n_{1}$ is reached when all photons are scattered backwards:

$$
F_{\text {scatter }}^{\max }=\frac{d p}{d t}=\frac{d p}{d N} \cdot \frac{d N}{d t}=\frac{h \nu}{c / n_{1}} \cdot \frac{P}{h \nu}=\frac{n_{1} P}{c} .
$$

This "scattering force" is the predominant factor far from any focal point of the beam. If, however, the laser beam is tightly focused, the highly convergent light rays close to the focus give rise to an additional force component known as the "gradient force". If the gradient force exceeds the scattering force, the stable optical trapping of particles is possible. To achieve this criterion, very steep gradients are necessary. Therefore objectives with a high numerical aperture are used to build optical traps.

The theoretical description of the gradient force depends on the size of the trapped particle. For particles with dimensions $d$ larger than the wavelength $\lambda$ of the laser, diffraction results in a force mainly perpendicular to the beam: a particle smaller than the wavelength will experience a force if it is polarizable. The electric field vector of the beam polarizes the particle in the direction of its own polarization, forming a dipole-dipole interaction between beam and particle. These two trapping regimes will be more closely examined in the following section.

\section{Ray-optics regime}

For a phenomenological understanding of a laser trap in the regime where Miescattering dominates (i.e. if $d \gg \lambda$ ), particles can be regarded as small lenses. When the focus of the laser is below the particle, the outgoing light gets more spread out than the incoming light. The resulting gradient force pushes the particle towards the focus (Figure 2.1 A). Similarly, the outgoing light gets more collimated if the focus is above the particle and the resulting momentum transfer opposes the

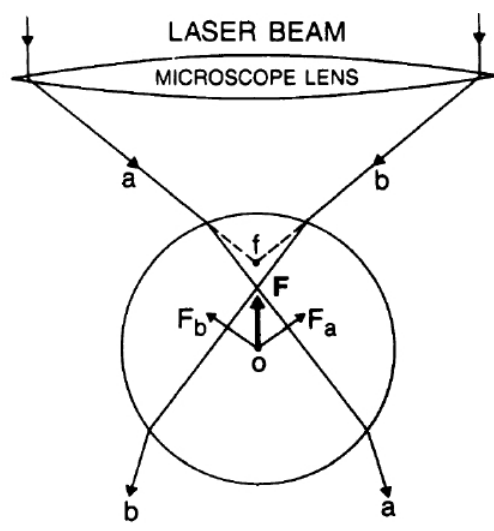

A

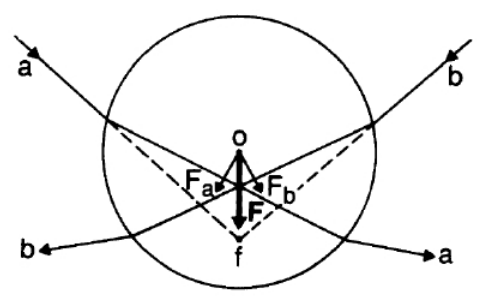

B

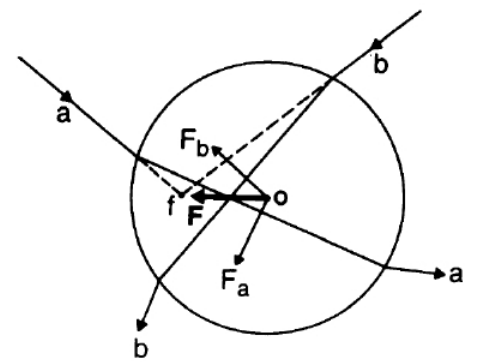

C

Figure 2.1: Ray-optics description of particle trapping

Light coming from a laser is focused by a microscope objective (focal point $f$ ). The light rays $a$ and $b$ are refracted when they impinge on a spherical object (circle with center $O$ ) that is displaced axially or transversely from the focus. The refraction of the beams causes a change of momentum which results in a force acting on the object. The sum of the forces $F_{a}$ and $F_{b}$ is always directed towards the focus. Figure adapted from [73]. 
Figure 2.2: Single beam of light entering an ideal sphere

A light beam with intensity $P$ passes through a particle. The intensity is decreased by the Fresnel coefficients $\mathrm{R}$ and $\mathrm{T}$ at each reflection. All reflections are at identical angles. Note that $\theta_{i}=\theta_{r}$, $\alpha=2\left(\theta_{i}-\theta_{t}\right)$ and $\beta=\pi-2 \theta_{t}$. Figure adapted from [73].

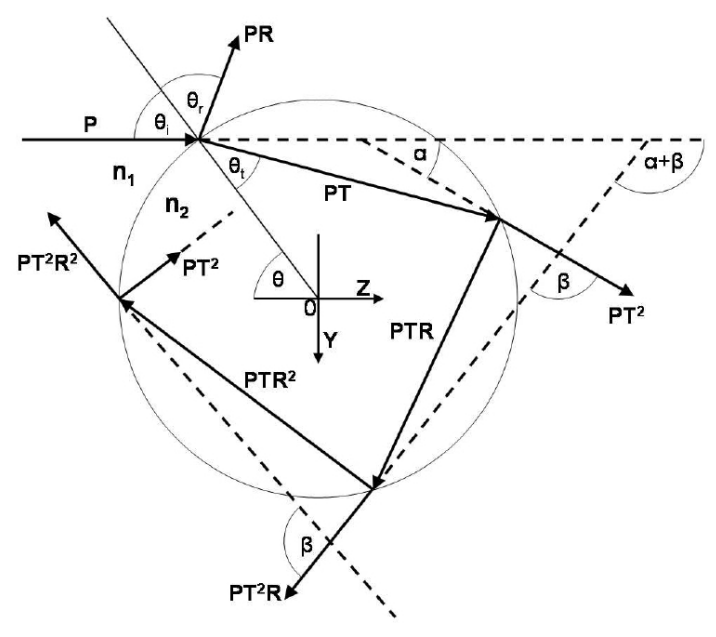

scattering force (Figure $2.1 \mathrm{~B}$ ). A force perpendicular to the illumination is achieved by deflection of the beam in the direction of the bead, giving a restoring force in the direction of the focus (Figure 2.1 C).

If a light beam passes through a particle with refractive index $\mathrm{n}_{2}$ that is different from the index of refraction of the surrounding medium $n_{1}$, its intensity is decreased by a factor given by the Fresnel coefficients $\mathrm{R}$ and $\mathrm{T}$ at each reflection. All reflections in the bead are at identical angles (Figure 2.2). For a spherical dielectric (nonabsorbing) particle, the Fresnel coefficients for light polarized perpendicular $\left(R_{\perp}\right.$ and $\left.T_{\perp}\right)$ and parallel $\left(R_{\|}\right.$and $\left.T_{\|}\right)$to the surface are given by

$$
\begin{aligned}
R_{\perp}=\frac{\sin \left(\theta_{i}-\theta_{t}\right)}{\sin \left(\theta_{i}+\theta_{t}\right)} & R_{\|}=\frac{\tan \left(\theta_{i}-\theta_{t}\right)}{\tan \left(\theta_{i}+\theta_{t}\right)} \\
T_{\perp}=\frac{2 \sin \theta_{t} \cos \theta_{i}}{\sin \left(\theta_{i}+\theta_{t}\right)} & T_{\|}=\frac{2 \sin \theta_{t} \cos \theta_{i}}{\sin \left(\theta_{i}+\theta_{t}\right) \cos \left(\theta_{i}-\theta_{t}\right)},
\end{aligned}
$$

with $\sin \theta_{i} / \sin \theta_{t}=n_{2} / n_{1}$.

The force, resulting from a light beam passing through a particle with refractive index $\mathrm{n}_{2}$, can be calculated directly [73]. The forces in perpendicular (gradient) and parallel (scatter) directions towards the beam can be written in terms of the force for total reflection (eq. (2.1)) as:

$$
\begin{aligned}
& F_{\|}=\frac{n_{1} P}{c}\left[1+R \cos 2 \theta_{i}-\sum_{j=0}^{\infty} T^{2} R^{j} \cos (\alpha+j \beta)\right] \\
& F_{\perp}=\frac{n_{1} P}{c}\left[R \sin 2 \theta_{i}-\sum_{j=0}^{\infty} T^{2} R^{j} \sin (\alpha+j \beta)\right],
\end{aligned}
$$

Setting $F_{t o t}=F_{\|}+i F_{\perp}$, the sums in eq. (2.2) can be simplified in the complex plane. The last term then becomes

$$
\sum_{j=0}^{\infty} R^{j} \sin (\alpha+j \beta)=\frac{\exp i \alpha}{1-R \exp i \beta}=\frac{\exp i \alpha+R \exp 2 i \theta_{i}}{1+\cos 2 \theta_{t}+R^{2}}
$$


where $\alpha=2\left(\theta_{i}-\theta_{t}\right)$ and $\beta=\pi-2 \theta_{t}$ was used (Figure 2.2).

The scattering and gradient forces for a single ray of light can now be written as follows:

$$
\begin{aligned}
& F_{\|}=\frac{n_{1} P}{c}\left[1+R \cos 2 \theta_{i}-T^{2} \cdot \frac{\cos \left(2\left(\theta_{i}-\theta_{t}\right)\right)+R \cos 2 \theta_{i}}{1+R^{2}+2 R \cos 2 \theta_{t}}\right] \\
& F_{\perp}=\frac{n_{1} P}{c}\left[R \sin 2 \theta_{i}-T^{2} \cdot \frac{\sin \left(2\left(\theta_{i}-\theta_{t}\right)\right)+R \sin 2 \theta_{i}}{1+R^{2}+2 R \cos 2 \theta_{t}}\right] .
\end{aligned}
$$

The factors in brackets are the Q-factors, $Q_{s}=F_{\|} c /\left(n_{1} P\right)$ and $Q_{g}=F_{\perp} c /\left(n_{1} P\right)$. The total force exerted on a particle by the trap can now be obtained by integration over all rays. This integration is carried out for a variety of beam profiles, polarizations and displacements of the particles in the work of Ashkin [73].

\section{Rayleigh regime}

For particles with dimensions much smaller than the laser wavelength $(d \ll \lambda)$, the ray optic calculations do not hold. Instead, the electric dipole moment induced by the laser light is considered. Again, the total force can be split into two components, a scattering force (dependent on the effective cross section of the particle $\sigma$ and the Poynting vector $S=E \times B$ ) and a gradient force directed towards the intensity maximum of the trap (dependent on the polarizability of the particle $\alpha$ and the gradient of the mean square electric field, $\left.\left\langle E^{2}\right\rangle\right)$ :

$$
\begin{aligned}
F_{\text {scatter }} & =\frac{n_{1}}{c}\langle S\rangle \sigma \\
F_{\text {grad }} & =\frac{\alpha}{2} \nabla\left\langle E^{2}\right\rangle .
\end{aligned}
$$

Both $\alpha$ and $\sigma$ can be written in terms of $m \equiv n_{2} / n_{1}$, the particle diameter $r$ and the wave vector $k=2 \pi n_{1} / \lambda$ :

$$
\alpha=n_{1}^{2} r^{3}\left(\frac{m^{2}-1}{m^{2}+2}\right)^{2} \quad, \quad \sigma=\frac{8}{3} \pi(k r)^{4} r^{2}\left(\frac{m^{2}-1}{m^{2}+2}\right)^{2} .
$$

We can obtain the Q-factor, which is defined:

$$
\begin{gathered}
Q=\frac{F_{\text {grad }}}{F_{\text {scatter }}}=\frac{3 \sqrt{3}}{64 \pi^{5}} \frac{n_{2}^{2}}{\left(\frac{m^{2}-1}{m^{2}+2}\right)} \frac{\lambda^{5}}{r^{3} \omega_{0}^{2}}, \\
\text { with } \quad \omega_{0}=\frac{\lambda}{\pi N A} .
\end{gathered}
$$

$\omega_{0}$ is the width of a Gaussian beam. It is obvious that a high numerical aperture $\mathrm{NA}$ is inevitable to obtain a stable trap with $Q \gg 1$. It should also be noted that the Q-factor scales with $1 / r^{3}$, which makes the trap more stable for small particles. 
A objective

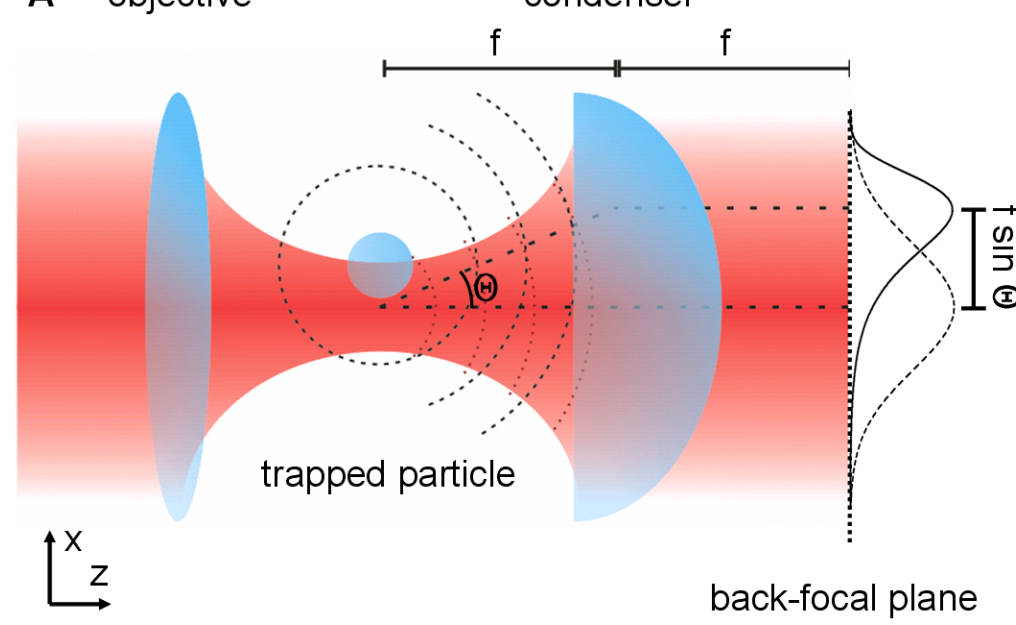

B quadrant photo diode
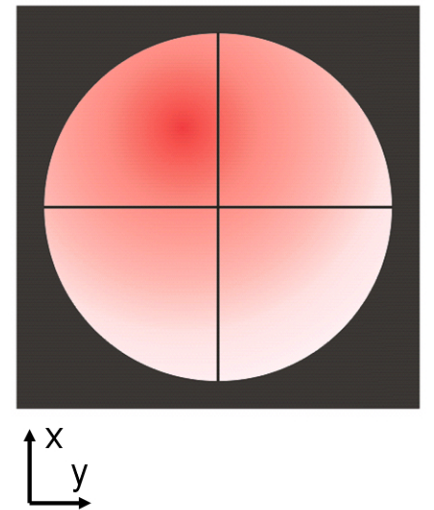

Figure 2.3: Back-focal-plane interferometry

A Lateral displacement of a trapped particle from the trap center is shown. Scattered and transmitted light are collected by the condenser and far-field interference results in a shift of the intensity distribution in the back-focal-plane. B This plane is imaged onto a quadrant diode, allowing a lateral resolution of the displacement down to the Å-regime. Figure based on [74].

\section{Back-focal-plane interferometry}

For most applications of optical traps it is not enough to just trap a particle, but it is also necessary to measure its position relative to the trap with very high spatial and temporal resolution. In the ray optics regime, the light is diffracted which is required to form a trap. For small particles, a restoring force arises from the momentum transfer of the incoming light to the bead, leading to a differently directed outgoing beam. In both cases, trapping and deflection are mutually dependent. This can be used to perform an interferometric measurement, based on back-focal-plane detection, to acquire the position of a trapped particle [74].

Gittes and Schmidt [74] computed a formula for the angular intensity distribution of a Gaussian beam passing a Rayleigh particle that is displaced from the focus perpendicular to the optical axis by a distance $x$. For viewing angles $\theta$ and $\phi$, where $\theta$ is in the direction of $x$, they obtained:

$$
\frac{I(x)-I(0)}{I_{\text {tot }}}=\frac{2 k^{3} \alpha}{\pi r^{2}} \exp \left(-\frac{x^{2}}{\omega_{0}^{2}}\right) \cdot \sin (k x \sin \theta \cos \phi) \exp \left(-k^{2} \omega_{0} 2^{2} \theta^{2} / 4\right)
$$

with intensity I and width $\omega_{0}$ of the Gaussian beam. $k=2 \pi n_{1} / \lambda$ denotes the wave vector. Pralle et al. [75] extended this calculation to include the axial position of the trap. They found a similar expression where the angular intensity pattern change for a displacement along the optical axis is also given, allowing particle tracking in three dimensions. Throughout this thesis, only two-dimensional data has been recorded.

In the actual experiment, the light of the trapping laser is collected by the condenser after it passes the sample and the trapped object. Unscattered light and light scattered by the trapped particle interfere. A lateral displacement of the 
particle in the specimen plan leads to a shift of the intensity profile on the back-focalplane of the condenser (Figure 2.3 A). This interference pattern does not depend on the relative position of the trap in the specimen plane but solely on the position of the particle in the focal volume of the trap. Thus, repositioning of the trap during experiments without changing any of its properties is possible. The interference pattern is imaged onto a quadrant photodiode (Figure $2.3 \mathrm{~B}$ ). The signals from the quadrants are summed pairwise and the differential signal is calculated from both pairs in the $\mathrm{x}$ - and $\mathrm{y}$-directions. This differential signal is normalized by the sum signal of the whole QPD [74]. For a quadrant photodiode, the intensity change due to a displacement, $\mathrm{x}$, of the trapped bead is given by [74]:

$$
\begin{aligned}
\Delta I_{x} & =\frac{I_{x+}-I_{x-}}{I_{x+}-I_{x-}}=\frac{16 k \alpha}{\sqrt{\pi} \omega_{0}^{2}} G\left(x / \omega_{0}\right), \\
G(u) & =\exp \left(-2 u^{2}\right) \int_{0}^{u} \exp \left(y^{2}\right) d y
\end{aligned}
$$

where $\mathrm{G}$ is the Dawson integral. In practical work with a laser trap, displacements are usually small. For small displacements, the QPD response function can be taken to be linear with no cross talk between channels: $\Delta I_{x}(x, y)=c \cdot x$, with the calibration coefficient $c$. Section 2.1.3 describes the methods of obtaining this calibration coefficient.

\subsubsection{Calibration of optical traps}

To measure forces, the detector response of an optical trap has to be calibrated. This can be done by applying known forces to a trapped particle. One elegant approach is to make use of the ever-present Brownian motion. The continuous and random bombardment by solvent molecules gives rise to diffusion of a free particle. The Langevin equation is the equation of motion for a small particle in a harmonic potential in a viscous medium with friction coefficient $\gamma$, driven by the thermal force $F(t)$. Here, the Langevin equation is ${ }^{1}$ :

$$
F(t)=\gamma \frac{d x(t)}{d t}+\kappa x(t)
$$

For a purely viscous medium, $F(t)$ arises from random elastic collisions that are statistically independent, i.e. white noise. The autocorrelation of this force is:

$$
\left\langle F(t) F\left(t+t^{\prime}\right)\right\rangle_{t}=C \cdot \delta\left(t^{\prime}\right)
$$

where $C$ is a real constant. Calculating the Fourier transform $\mathcal{F}$ of the autocorrelation, the power spectrum is:

$$
|F(\omega)|^{2}=C \cdot \mathcal{F}[\delta(t)]=C
$$

\footnotetext{
${ }^{1}$ Since the Reynolds number is very low $\left(10^{-4}\right)$ for micrometer sized object, inertial forces have been neglected.
} 
This fact leads us directly to the particle's power spectrum, which is a Lorentz function (see Gittes and Schmidt, [76]):

$$
\operatorname{PSD}(\omega)=\frac{1}{\tau}|x(\omega)|^{2}=\frac{C \tau^{-1} \gamma^{-2}}{\omega^{2}+(\kappa / \gamma)^{2}}=\frac{A}{\omega^{2}+\omega_{c}^{2}}
$$

Here, the critical frequency $\omega_{c}=\kappa / \gamma$ and the total duration $\tau$ of the signal $x(t)$ are introduced. To transform the energy spectrum $|x(\omega)|^{2}$ into a power spectrum independent of the total duration of the measurement, the normalization to the duration of the measurement is needed. Mathematically $\tau$ has to be infinite. However, the discrete Fourier transform for finite signals approximates the continuous Fourier transform of infinite signals to a sufficient degree. The amplitude of the driving force $C$ is calculated by comparing thermal and mechanical forces and using Parseval's theorem:

The equipartition theorem for the harmonic potential $U=1 / 2 \kappa x^{2}$ leads to

$$
\frac{1}{2} k_{B} T=\frac{1}{2} \kappa\left\langle x^{2}(t)\right\rangle
$$

It follows:

$$
\begin{aligned}
\frac{k_{B} T}{\kappa} & =\left\langle x^{2}(t)\right\rangle=\frac{1}{2 \pi} \int_{-\infty}^{\infty} d \omega \frac{1}{\tau}|x(\omega)|^{2}=\frac{1}{2 \pi} \int_{-\infty}^{\infty} d \omega \frac{A}{\omega^{2}+\omega_{c}^{2}} \\
& =\frac{A}{2 \pi \omega_{c}} \int_{-\infty}^{\infty} d \xi \frac{1}{1+\xi}=\frac{A}{2 \omega_{c}} .
\end{aligned}
$$

This relation yields an expression for $\mathrm{A}$ and thus the amplitude for the thermal force:

$$
\begin{aligned}
|F(\omega)|^{2} & =C=A \tau \gamma^{2}=2 k_{B} T \omega_{c} \tau \gamma^{2} / \kappa=2 k_{B} T \tau \gamma \\
\Rightarrow A & =\frac{2 k_{B} T}{\gamma} .
\end{aligned}
$$

From the quadrant photo diodes of the setup the power spectrum of the particles movement can be gained in volts:

$$
\frac{1}{\tau}\left|u_{e l e c}\right|^{2}=\frac{A_{V}}{\omega^{2}+\omega_{c}^{2}}
$$

which is related to the power spectrum of the bead's trajectory,

$$
\frac{1}{\tau}\left|u_{m e c h}\right|^{2}=\frac{2 k_{B} T}{\gamma\left(\omega^{2}+\omega_{c}^{2}\right)}
$$

by $u_{\text {elec }}[V] \cdot c[m / V]=u_{\text {mech }}[m]$. With the Stokes drag coefficient, $\gamma=6 \pi \eta R$, this leads to:

$$
c=\sqrt{\frac{2 k_{B} t}{6 \pi \eta R A_{V}}} .
$$


The values for $\omega_{c}$ and $A_{V}$ can be obtained by fitting the theoretically derived Lorentz function to the experimentally recorded data. With these values, the calibration factor $\mathrm{c}$ as well as the trap stiffness $\kappa=6 \pi \eta R \omega_{c}$ can be calculated.

\section{$2.2 \quad$ The setup}

The optical trap used in this thesis is integrated by a custom built inverted microscope (Figure 2.4). For optical trapping, a linear polarized near-infrared solid-state laser (Compass, Nd:YVO 4,1064 nm, 4 W, Coherent Inc., Santa Clara, CA, USA) is used, protected against back reflections by an optical isolator (IO-5-1064-VHP, Thorlabs, Newton, NJ, USA). The diameter of the beam is expanded by a $3 \mathrm{x}$ beam expander (Qioptiq Photonics GmbH \& Co KG, München, Germany) to a size of $\sim 4 \mathrm{~mm}$. To allow the running of the laser at high power for increased stability, a combination of a motor driven rotatable half-wave plate $(\lambda / 2)($ PRM1Z8, Thorlabs, Newton, NJ, USA) and a polarizer is used to adjust the laser intensity. A Glan-Laser polarizing beam splitter (BS 1) is used to split the laser into two orthogonally polarized beams which will form two individually adjustable laser traps in the specimen plane. The ratio of the power of the two beams can be adjusted by changing the orientation of the input polarizer. In both beams a 1:1 telescope is inserted which allows changing the position of the trap in the sample. The beam profile from the focal plane of the first telescope lens is imaged on the back-focal-plane (BFP) of the objective by the second telescope lens. This allows for beam steering without changing the intensity distributions in the BFP of the objective, as well as in the conjugated condenser BFP. A second Glan-Laser polarizing beam splitter (BS 2) recombines the two beams before they are coupled into the microscope by a dichroic mirror.

The laser beam is focused into the sample by a 100x magnification, infinity-corrected, 1.3-NA oil immersion objective (Neofluor, Carl Zeiss MicroImaging GmbH, Jena, Germany). After passing the sample, the light is collected by a 1.4-NA oil immersion condenser lens (Carl Zeiss MicroImaging GmbH, Jena, Germany) and again split into the orthogonally polarized beams by a polarizing beam splitter (BS 3). The light distribution in the condenser's BFP for both of the polarized beams is imaged by another set of lenses onto two quadrant photodiodes (QPDs). The four photocurrent signals of each diode are converted to voltages and combined to measure the intensity distributions for the two lateral directions $\mathrm{x}$ and $\mathrm{y}$ in the BFP (see also 2.1.2).

For optical microscopy, two different illuminations can be used at the same time. Bright field imaging is possible using a LED that is coupled into the microscope from the top. A $532 \mathrm{~nm}$ diode laser (30 mW, Viasho, Beijing, China) is coupled into the microscope below the trapping laser to enable epi-fluorescence illumination. For both methods, the sample is imaged onto a CCD-camera (Coolsnap EZ, Photometrics, Tucson, AZ, USA). 


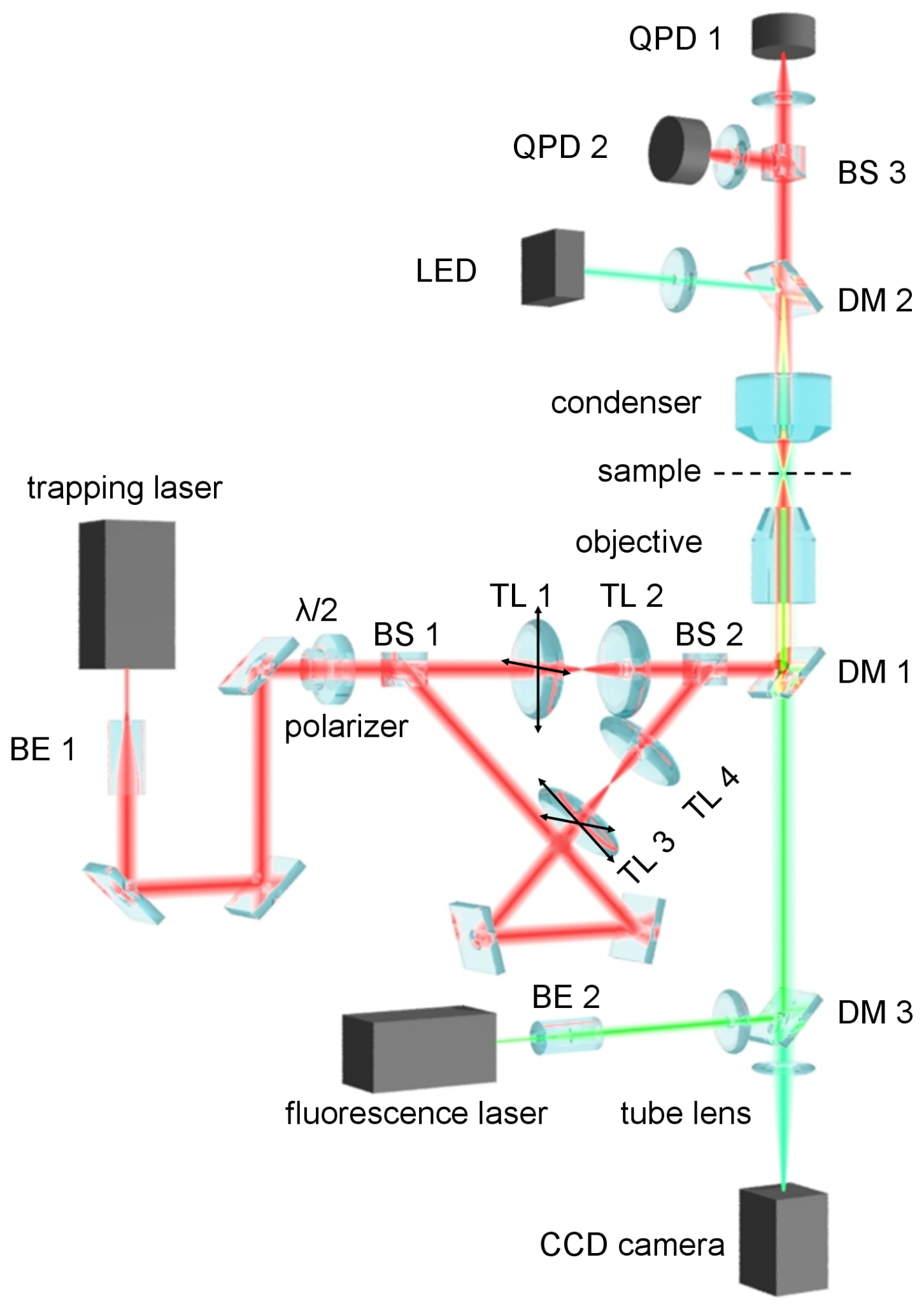

Figure 2.4: Schematic layout of the setup

The $1064 \mathrm{~nm} \mathrm{Nd:YVO} \mathrm{trapping} \mathrm{laser} \mathrm{passes} \mathrm{through} \mathrm{a} \mathrm{beam} \mathrm{expander} \mathrm{(BE),} \mathrm{a} \mathrm{half-wave} \mathrm{plate}$ and a polarizing beam splitter (BS). The beam splitter splits the beam into two orthogonally polarized beams, which later form two individual traps in the specimen plane. The beams are recombined by a second beam splitter and coupled into the microscope via a dichroic mirror (DM). After passing the objective, the laser is again split by a beam splitter and imaged onto the QPDs. LED illumination for bright field microscopy is coupled in from above and an additional $532 \mathrm{~nm}$ laser for epi-fluorescence microscopy from below the sample. 


\subsection{Experimental approaches}

For the work presented in this thesis, numerous experimental approaches are recurring. The following sections present the assays which are most extensively used. For more detailed instructions on how to prepare some of the components, e.g. coating of cover slips or different microtubule variants, refer to Appendix A. If not otherwise indicated, all chemicals and materials were obtained from Sigma-Aldrich Chemie Gmbh (Munich, Germany).

\subsubsection{Single-bead motility assay}

A single-bead motility assay presents a method to measure the molecular forces and the velocities of motor proteins. To be able to detect the forces, which are typically in the range of a few piconewtons, and the velocities, that are in the range of several nanometers per second, optical traps are the ideal instruments of choice. Micronsized glass spheres, coated with motor proteins, are captured with an optical trap and placed on top of a microtubule (Figure 2.5). The motors start to pull the bead out of the laser focus. This motion is recorded with optical trap. From the initial slope of this displacement, the velocity is gained, while the maximum displacement, the motor reaches prior to unbinding, can be calculated to a force. Additionally, when polarity-marked microtubules are used, the directionality of the motion can be studied.

Exemplary data from a single-bead motility assay is shown in Figure 2.6. If the microtubule, on top of which the motor-coated bead was positioned, was not aligned with either the $\mathrm{x}$ - or $\mathrm{y}$-axis of the setup, the motion of the bead was divided between the $\mathrm{x}$ - and $\mathrm{y}$-channel (Figure $2.6 \mathrm{~A}$ ). To convert the signal into one channel, we performed a principle component analysis to calculate the angle between the actual motion and the setup-axes. To gain the motile properties of the protein from the data, we rotated the data set by the calculated angle which resulted in two signals, one parallel and the other orthogonal to the motion of the bead (Figure 2.6 B). The initial slope of the movement of the bead was then linearly fitted to obtain the velocity. The force was calculated by multiplying the maximum displacement of the bead prior to an unbinding event with the trap stiffness.

Plain silica beads with a diameter of one micrometer (Kisker Biotech $\mathrm{GmbH} \&$ Co. KG, Steinfurt, Germany) in BRB80 (80 mM PIPES/KOH, pH 6.8, $1 \mathrm{mM}$ $\mathrm{MgCl}_{2}, 1 \mathrm{mM}$ EGTA) were incubated for $30 \mathrm{~min}$ with $50 \mu \mathrm{g}$ protein-G. After centrifugation and re-suspension, the beads were incubated with $1 \mu \mathrm{g}$ penta-hisantibody and $0.02 \mathrm{mg}$ casein. After $30 \mathrm{~min}$, the beads were centrifuged and resuspended to the desired concentration. Diluted his-tagged motor proteins were then added to the beads and allowed to bind for $15 \mathrm{~min}$.

A sample chamber was constructed using plasma cleaned (plasma cleaner from Harrick Plasma, Ithaca, NY, USA) cover slips, silanized with a positively-charged silane 3-[2-(2-Aminoethylamino)-ethylamino]propyl-trimethoxysilane (DETA), and 


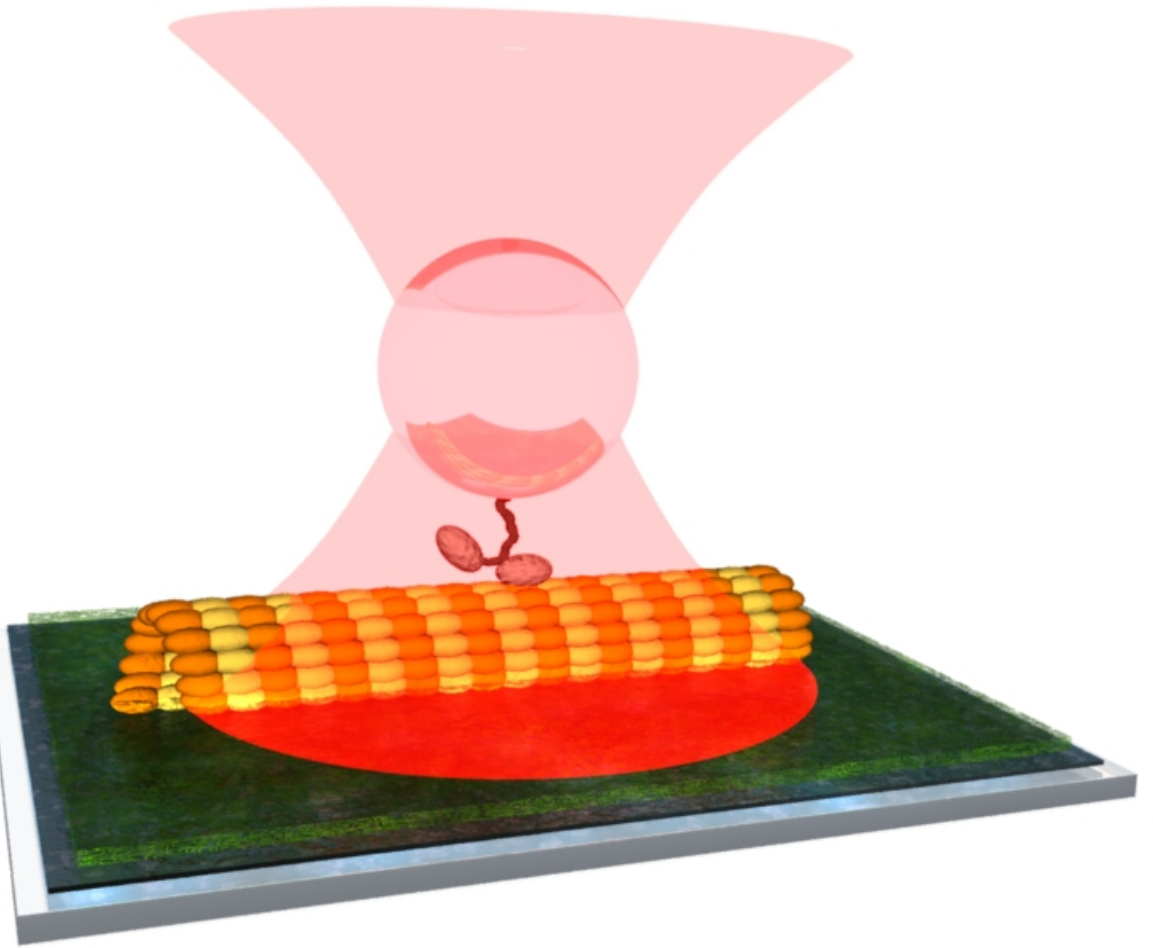

Figure 2.5: Single-bead motility assay

A micro sphere, coated with motor proteins, is trapped with an optical trap and positioned above a surface-immobilized microtubule. When the motor binds to the microtubule it starts to displace the bead relative to the trap. The motion of the bead is recorded with the optical trap.

A

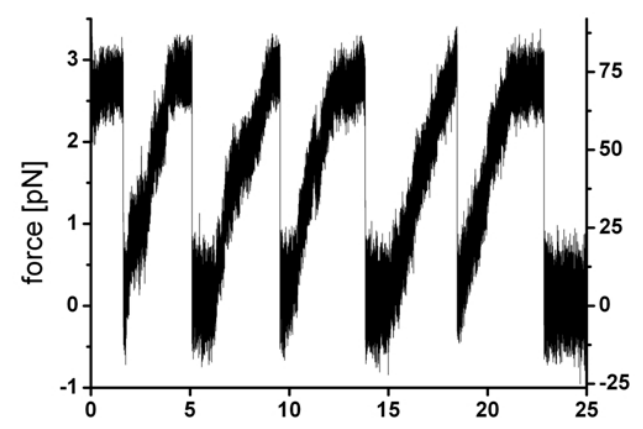

y-channel
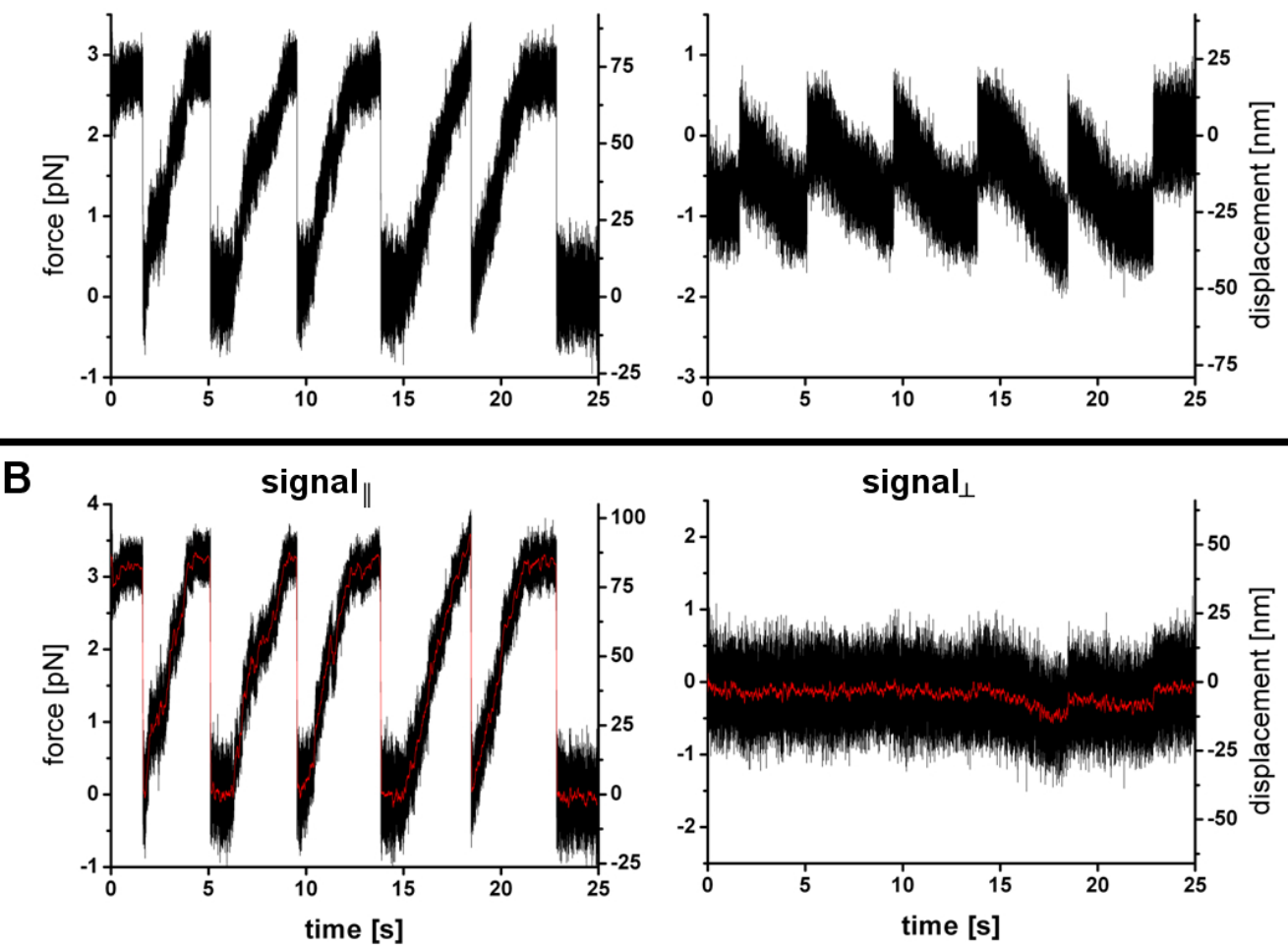

signal $_{\perp}$

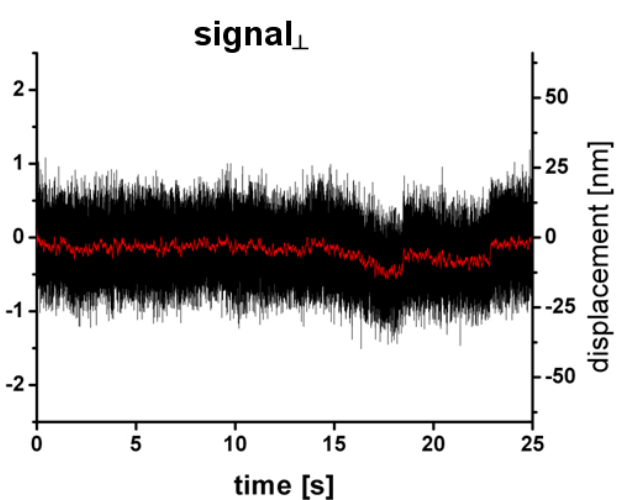

Figure 2.6: Exemplary data from a single-bead motility assay

A Raw data of a single-bead assay with Eg5Kin NL-14. B After post-processing, the data is divided into signals parallel and orthogonal to the motion of the bead. The median filtered data (0.03 s sliding window) is shown in red. Trap stiffness: $0.038 \mathrm{pN} / \mathrm{nm}$. 
double-sided tape. Tetramethylrhodamine- (TMR)-labeled microtubules were filled into the chamber and allowed to bind to the DETA surface for $5 \mathrm{~min}$. To prevent the beads from later sticking to the surface, the chamber was sealed by adding a solution containing $0.1 \mathrm{mg}$ casein to the chamber which was incubated for a minimum of 5 min. The chamber was then flushed with assay buffer (BRB80 containing $2 \mathrm{mM}$ ATP, $4 \mathrm{mM} \mathrm{MgCl}_{2}$, and an oxygen scavenging system based on $10 \mathrm{mM} \mathrm{DTT}$, $0.08 \mathrm{mg} / \mathrm{ml}$ catalase $40,0.1 \mathrm{mg} / \mathrm{ml}$ glucose oxidase and $10 \mathrm{mM}$ glucose), containing the motor-coated beads.

Beads were trapped using a focused 4 W 1064 nm NdYVO laser (Coherent Inc., Santa Clara, CA, USA) in a custom-built single-beam optical trap described in Section 2.2. After positioning a bead above a microtubule, the motion of the bead was measured with the optical trap by back-focal-plane interferometry (see Section 2.1.2). This data was acquired and recorded with custom-written LabView (National Instruments, Austin, Texas, USA) software. Further analysis and statistical calculations were performed with MatLab (The Mathworks, Natick, MA, USA) and OriginPro (OriginLab Corporation, Northampton, MA, USA). All measurements were done at $22^{\circ} \mathrm{C}$.

To prevent multiple motors on the bead from binding to the microtubule at the same time, the concentration of the motor was adjusted so that less than half of the beads showed any activity in the experiment. Assuming a Poisson probability, this ensured that only single proteins were interacting with a microtubule at any given time $[7,77,78]$.

\subsubsection{Single-molecule fluorescence assay}

In a single-molecule fluorescence assay, single fluorescently labeled motor proteins are recorded while moving along a microtubule using a total-internal-reflection fluorescence (TIRF) microscope (Figure 2.7; for details on the TIRF microscope, see [46]). This assay complements a single-bead motility assay. Where the latter provides force and velocity of a molecular motor, the single-molecule fluorescence assay allows for measuring the velocity as well as the run length of the motor. This does not only give us two independent and comparable methods to obtain the velocity, but also completes our knowledge of the motile properties by adding the run length. Another variant of the single-molecule fluorescence assay is the relative-sliding assay. When molecular motors that are able to processively crosslink two microtubules are studied (e.g. mitotic motors, see Section 1.2), additional microtubules are added to the motility buffer. Now the motility of the motors themselves as well as the motion of potentially crosslinked microtubules can be monitored (Figure 2.8). For this assay, as well as the standard single-molecule fluorescence assay, the use of polarity-marked microtubules allows for studies of the directionality of the motor's movement.

Exemplary data from a single-molecule fluorescence assay is shown in Figure 2.9. During the experiment, we recorded videos of the GFP-labeled motors moving along TMR-labeled microtubules that were immobilized on the surface. Both fluorescence signals were recorded simultaneously and afterwards merged with ImageJ. From 


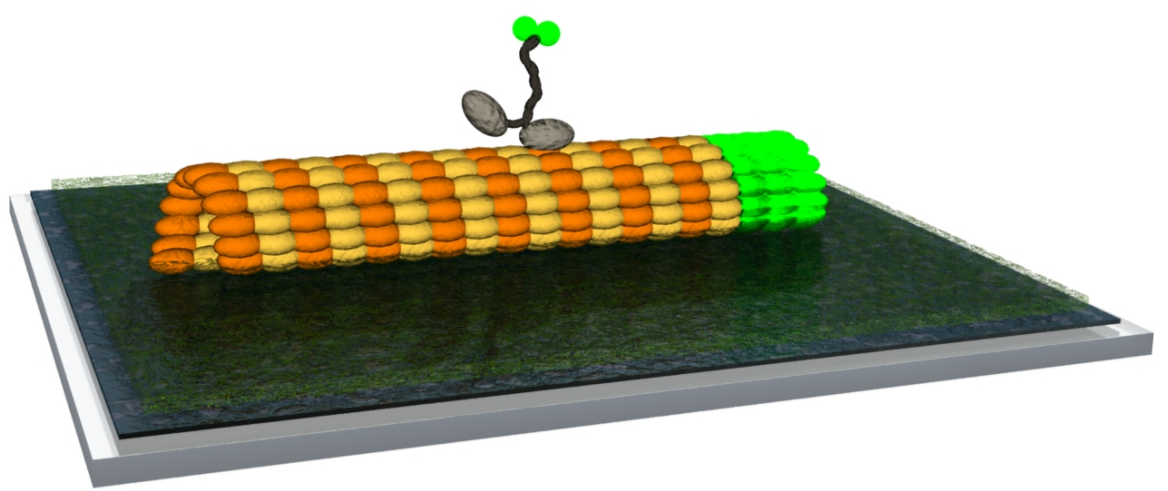

Figure 2.7: Single-molecule fluorescence assay

Fluorescently labeled microtubules are immobilized on the surface of a measurement chamber. A solution containing molecular motors labeled with a different fluorescent dye is flushed into the chamber. With a TIRF microscope, the motion of individual motor proteins along single microtubules can be observed.

the videos, kymographs along single microtubules were obtained by using ImageJ macros written by J. Rietdorf and A. Seitz. The slope of the trace visible in Figure 2.9 provided us with the motors' velocity while the length of an individual trace corresponds to the motors' run length.

To immobilize microtubules, cover slips were silanized with the positively-charged silane DETA after cleaning using a plasma cleaner (Harrick Plasma, Ithaca, NY, USA). These DETA cover slips were used to assemble assay chambers by sticking them with double-sided tape on a microscope slide. For experiment preparation,

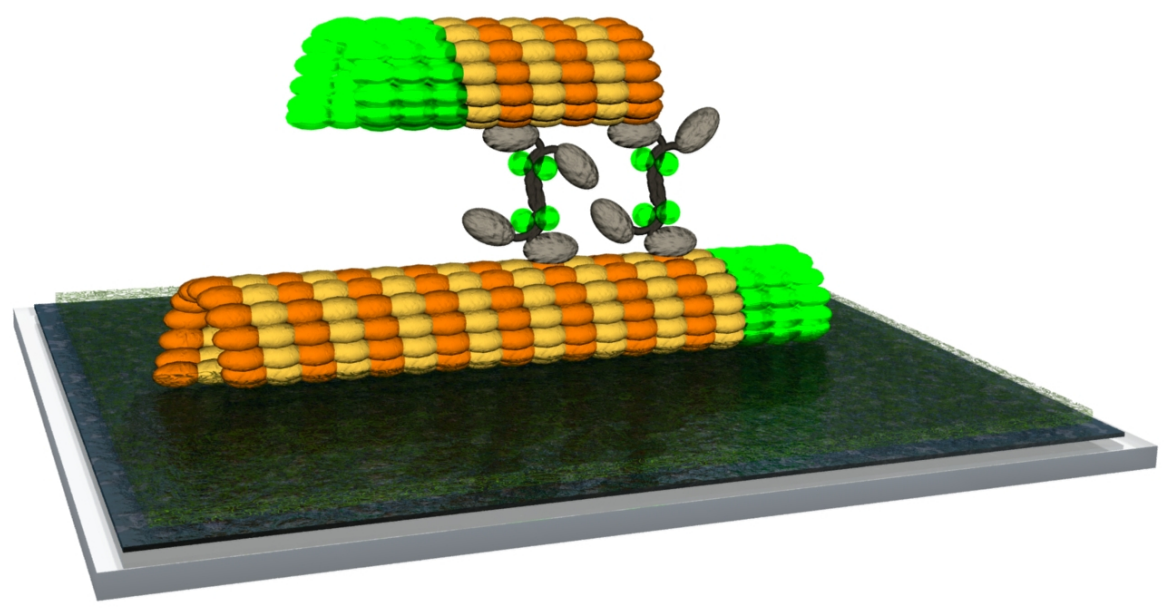

Figure 2.8: Relative-sliding assay

A variation of the single-molecule fluorescence assay is the relative-sliding assay. In this assay, small microtubules are added to the solution that contains the motors. If the motor proteins are able to processively crosslink the surface-bound microtubules and the microtubules in solution, relative sliding occurs. 

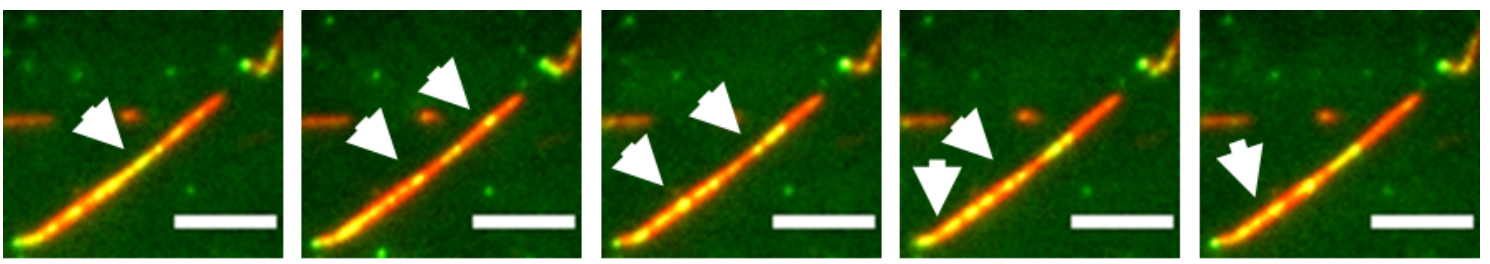

Figure 2.9: Exemplary data from a single-

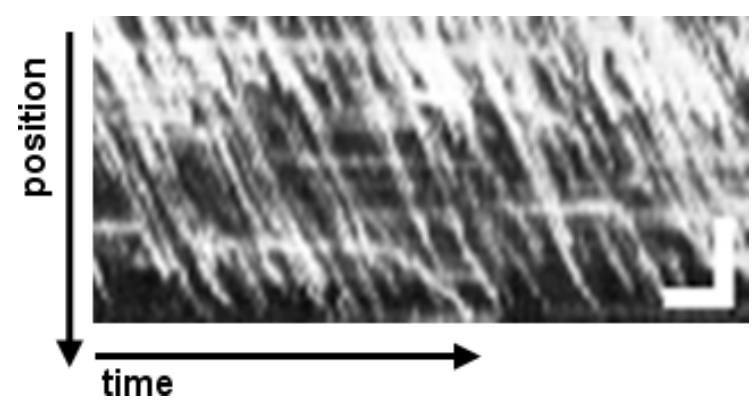
molecule fluorescence assay

Top: Image sequence from a single molecule fluorescence assay with DK4mer. Single GFP-labeled motor proteins (green) moving along surface-immobilized, TMR-labeled microtubules (red) are indicated. Scale bar: $5 \mu \mathrm{m}$. Left: Kymograph of the microtubule shown at the top. Traces of multiple moving motors can be seen. Vertical scale bars: 9 s; horizontal scale bars: $3 \mu \mathrm{m}$.

TMR-labeled microtubules with Atto-488-labeled minus ends, diluted in BRB80 buffer containing $10 \mu \mathrm{M}$ taxol (paclitaxel), were allowed to bind to the silanized glass surfaces of the assay chamber for $5 \mathrm{~min}$. This was followed by $5 \mathrm{~min}$ of incubation with $0.1 \mathrm{mg} / \mathrm{ml}$ casein in BRB80. The chamber was then flushed with assay buffer (BRB80 containing $2 \mathrm{mM}$ ATP, $4 \mathrm{mM} \mathrm{MgCl}_{2}$, and an oxygen scavenging system based on $10 \mathrm{mM}$ DTT, $0.08 \mathrm{mg} / \mathrm{ml}$ catalase 40, $0.1 \mathrm{mg} / \mathrm{ml}$ glucose oxidase and $10 \mathrm{mM}$ glucose) containing GFP-tagged motor proteins at appropriate singlemolecule concentrations. For relative-sliding assays, a similar preparation was used. In addition, short, polarity-marked microtubules were added to the motility buffer. In the actual experiment, fluorescence was observed in a custom-built TIRF microscope, using a 473-nm Laser (Coherent Inc., Santa Clara, CA, USA) for excitation of Atto-488 and a 532-nm Laser (Viasho, Beijing, China) for TMR , and a x100 objective (SFluor, NA 1.49, Oil, Nikon, Düsseldorf, Germany) and a CCD camera (iXon Ultra, Andor, Belfast, UK). Digital videos were recorded with Solis (Andor, Belfast, UK) and analyzed for motor speeds and run lengths using kymographs generated with open access ImageJ software (written by J. Rietdorf and A. Seitz). Statistical analysis of the data was performed with OriginPro (OriginLab Corporation, Northampton, MA, USA). All measurements were performed at $22{ }^{\circ} \mathrm{C}$.

\subsubsection{Surface-gliding assay}

A surface-gliding assay is a rather simple assay in which motor proteins are allowed to bind to the glass surface of an assay chamber. This either happens by unspecific or by anti-body binding. An assay buffer containing fluorescently labeled microtubules is then filled into the chamber. The microtubules are captured and pushed along the surface by the immobilized motors (Figure 2.10). The motion of the microtubules is recorded with an epi-fluorescence microscope. This assay is also called a 'multimotor gliding assay' since the velocity of an ensemble of motors can be obtained 


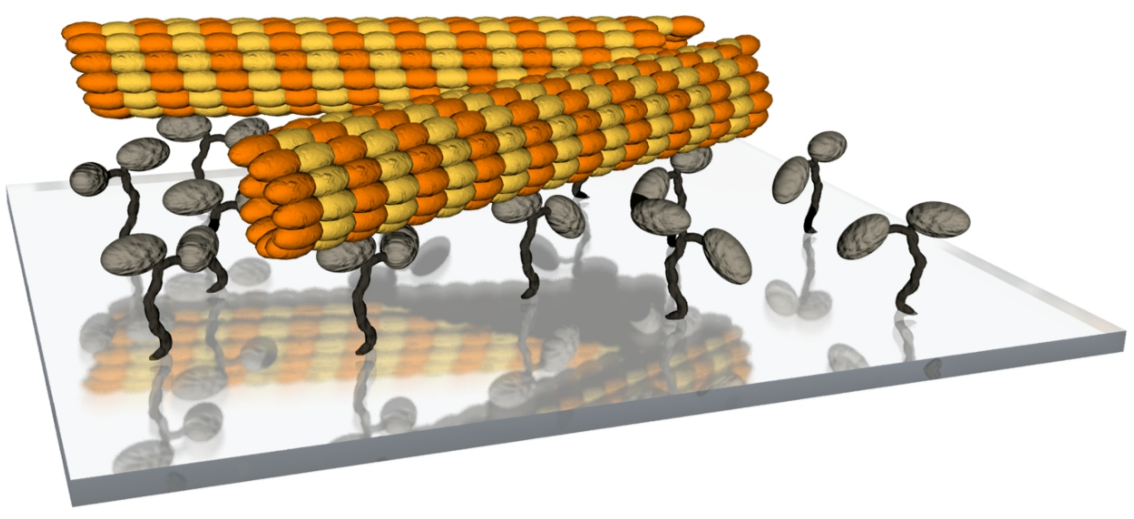

Figure 2.10: Surface-gliding assay

In a multi-motor or surface-gliding assay, the surface of a measurement chamber is coated with motor proteins. Afterwards, a solution containing microtubules is flushed into the chamber. The microtubules bind to the motors and are pushed along the surface.

with this experiment. One advantage is that the motility of non-processive motors can be visualized with this assay because the filaments stay bound to the surface as long as at least one motor stays attached.

Exemplary data from a surface-gliding assay is shown in Figure 2.11. Videos were recorded from the microtubules that were pushed along the surface by molecular motors attached to the surface of an assay chamber. The distance a microtubule traveled over a given time corresponds the velocity of the motor.

Sample chamber were made from plasma-cleaned cover slips and microscope slides assembled with double-sided tape. A solution containing the motor proteins was filled into the chamber and the motors were allowed to bind to the glass surfaces for 5 min. Subsequently, the chamber was flushed with assay buffer (BRB80 containing $10 \mu \mathrm{M}$ taxol (paclitaxel), $2 \mathrm{mM}$ ATP, $4 \mathrm{mM} \mathrm{MgCl}_{2}, 10 \mathrm{mM}$ DTT, $0.08 \mathrm{mg} / \mathrm{ml}$ catalase C40, $0.1 \mathrm{mg} / \mathrm{ml}$ glucose oxidase and $10 \mathrm{mM}$ glucose) containing $0.022 \mathrm{mg} / \mathrm{ml}$ tetramethylrhodamine (TMR)-labeled microtubules. Motility was observed in a standard inverted fluorescence microscope (Axiovert 200, Carl Zeiss MicroImaging GmbH, Jena, Germany). Images were recorded with a digital CCD camera (CoolSnap ES, Roper Scientific, Martinsried, Germany). All measurements were performed at $22{ }^{\circ} \mathrm{C}$.
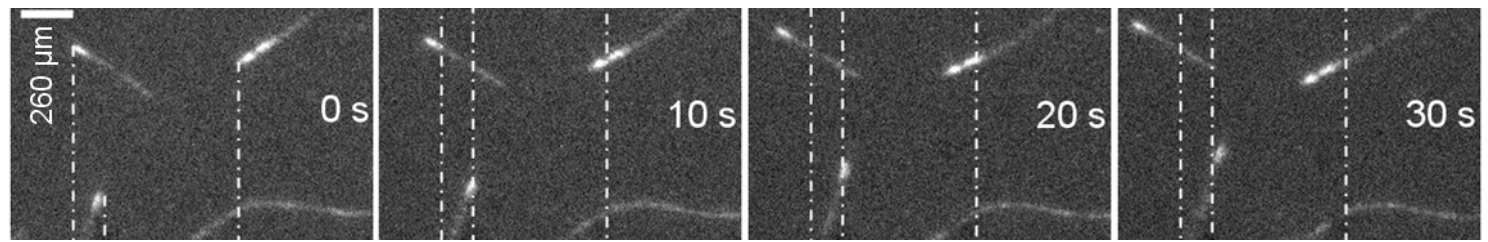

Figure 2.11: Exemplary data from a surface-gliding assay Image sequence from a surface-gliding assay with Eg5Kin. Polarity-marked microtubules are pushed along the surface of an assay chamber. The dashed lines indicate the microtubules' position at the beginning of the recording $(\mathrm{t}=0 \mathrm{~s})$. Figure adapted from Düselder et al. [79]. 


\subsection{Protein expression and purification}

In the following section, the essential procedures for expression and purification of the motor proteins that are used in this thesis will be given.

\section{Eg5Kin-NL constructs}

Based on Düselder et al. [79].

We constructed twelve chimeric motor proteins consisting of the motor domain and varying parts of the neck linker of Kinesin-5 Eg5 from Xenopus laevis and the stalk of conventional Kinesin from Drosophila melanogaster. The original Eg5Kin construct was generated as follows [68]: Eg5Kin was constructed from pPK113 (pET5a-DmKHC-His) and pBK006 (Eg5-GFP) using a nested PCR approach to extend the sequence of the Eg5 motor domain (residues 1-369) with the DmKHC residues starting at residue 345 towards an existing HindIII restriction site in pPK113. The DmKHC motor domain in pPK113 was subsequently replaced using NdeI and HindIII. The shortened GFP-tagged Eg5Kin-GFP was truncated at residues corresponding to DmKHC421, using PCR primers providing an AscI-XmaI site upstream of a stop codon. The full-length DmKHC in pPK113 was replaced with this truncated DmKHC421 using NdeI and NotI. A GFP-6His cassette flanked by AscI and XmaI was generated in pT7-7 for insertion downstream of the truncated Eg5Kin construct in pPK113.

Deletions or insertions of codons for the generation of the neck-linker constructs were performed in a two-step PCR approach. Using a complementary pair of modified primers in the neck-linker region, we created two PCR-products: one of the motor-domain up to the desired mutation and a second starting with the neck-linker mutation and ending with the C-terminal GFP. Both fragments were combined to serve as a template for a second round of PCR using the 3'- and 5'-primers flanking the $\mathrm{N}$ - and $\mathrm{C}$-terminus of the full construct. The resulting PCR products were then incorporated into pTOPO using a TOPO-Kit (Invitrogen, Carlsbad, CA, USA) for sequencing and amplification before replacing the coding sequence in pEG5KinGFPhis by sub-cloning using the existing restriction sites for NdeI and XmaI. Constructs NL-09, NL-12, NL-19, NL-20, NL-21 and NL-21P were generated by gene synthesis (GeneArt, Life Technologies, Regensburg, Germany). The integrity of the constructs was confirmed by sequencing.

Motors were expressed in E. coli and essentially purified as described in Lakämper et al. [68]. In brief, E. coli BL21(DE3) (Invitrogen, Carlsbad, CA, USA) were transformed and grown to a density of $\sim 0.6$ at $37^{\circ} \mathrm{C}$ before induction of expression using $1 \mathrm{mM}$ IPTG for $3 \mathrm{~h}$. Cells were harvested by centrifugation and resuspended in a $20 \mathrm{mM}$ imidazole buffer, $\mathrm{pH}$ 7.4, supplemented with $1 \mathrm{mM}$ each of DTT, $\mathrm{MgCl}_{2}$, EGTA, BME and $150 \mathrm{mM} \mathrm{NaCl}$ before lysis using ultrasound in the presence of lysozyme and DNAseI. After separation from cell debris by centrifugation, the cytosol was incubated for $1 \mathrm{~h}$ at $4^{\circ} \mathrm{C}$ with Ni-NTA-column material (Qiagen, Hilden, Germany) and then transferred to a syringe column. After washing the column with 
$80 \mathrm{mM}$ imidazole and $300 \mathrm{mM} \mathrm{NaCl}$, bound motors were eluted using a $300 \mathrm{mM}$ imidazole buffer containing $10 \mu \mathrm{M}$ ATP and $1 \mathrm{mM}$ DTT. Fractions containing motors were pooled and $3 \mathrm{x}$ dialyzed against $80 \mathrm{mM}$ PIPES/KOH, $1 \mathrm{mM} \mathrm{MgCl}$, $1 \mathrm{mM}$ EGTA, $1 \mathrm{mM}$ DTT, $10 \mu \mathrm{M}$ ATP. In addition, motors were also purified using a microtubule-affinity purification procedure described in [78]. Protein samples were then tested for motor activity and stored in aliquots at $-80^{\circ} \mathrm{C}$.

\section{Cin8Kin construct}

Based on Düselder et al. [79] and Gerson-Gurwitz et al. [46].

The Cin8Kin-Chimera was constructed from pPK113 pET5a-FL (DmKHC-his) and LGB830 pVL18 (Cin8-GFP) [46] using a nested-PCR approach in order to extend the sequence of the Cin8 motor domain (1-534) with the DmKHC residues starting at 345 towards an existing HindIII restriction site in pPK113. The DmKHC motor-domain in pPK113 was subsequently replaced by sub-cloning of this transition clone using NdeI and HindIII. The shortened GFP-tagged clone was truncated at residues corresponding to DmKHC 421, using PCR primers providing an AscI and XmaI site upstream of a stop-codon and replaced the FL-DmKHC in pPK113 using NdeI and NotI. A GFP-6his cassette flanked by AscI and XmaI was generated in pT7-7 for insertion downstream of the truncated Cin8Kin-construct in pPK113. The motor was expressed in E. coli and purified as described above.

\section{Eg5 wild-type protein}

Based on Korneev et al. [10] and Kapoor and Mitchison [80].

Full-length Eg5 with amino-terminal GFP and polyhistidine tag was expressed using a Bac-to-Bac system (Gibco, Life Technologies, Regensburg, Germany) in SF9 cells. $72 \mathrm{~h}$ after infection, the SF9 cells were harvested and the pellets were frozen in liquid nitrogen. Cell pellets were lysed $\left(50 \mathrm{mM} \mathrm{KPO}_{4}, 250 \mathrm{mM} \mathrm{KCl}\right.$, $10 \mathrm{mM}$ imidazole, $1 \mathrm{mM}$ PMSF, $10 \mathrm{mM}$ BME, $0.5 \mathrm{mM}$ MgATP, 1\% Igepal, protease inhibitors $[10 \mu \mathrm{M}$ leupeptin, $10 \mu \mathrm{M}$ pepstatin $\mathrm{A}, 10 \mu \mathrm{M}$ chymostatin], pH 8.0) and clarified (125,000 g, $45 \mathrm{~min}$ ) and the supernatant was incubated with Ni-NTA agarose (Qiagen, Hilden, Germany) in batch for $90 \mathrm{~min}$. The resin was then washed extensively with wash buffer $\left(10 \%\right.$ glycerol, $50 \mathrm{mM} \mathrm{KPO}_{4}, 250 \mathrm{mM} \mathrm{KCl,} 10 \mathrm{mM}$ imidazole, $1 \mathrm{mM}$ benzamidine, $10 \mathrm{mM}$ BME, $0.1 \mathrm{mM}$ MgATP, protease inhibitors, $\mathrm{pH}$ 8.0). The protein was eluted (10\% glycerol, $50 \mathrm{mM} \mathrm{KPO}_{4}, 150 \mathrm{mM} \mathrm{KCl,} 250 \mathrm{mM}$ imidazole, $1 \mathrm{mM}$ benzamidine, $10 \mathrm{mM}$ BME, $0.1 \mathrm{mM} \mathrm{MgATP}$, protease inhibitors, $\mathrm{pH}$ 7.0), dialyzed against superose buffer (10 mM Hepes, $150 \mathrm{mM}$ sucrose, $250 \mathrm{mM}$ $\mathrm{KCl}, 10 \mu \mathrm{M}$ MgATP, $1 \mathrm{mM}$ DTT, protease inhibitors, $\mathrm{pH}$ 8.0), and purified over a size exclusion column (Superose 6, GE Healthcare Bio-Sciences, Pittsburgh, PA, USA). The Protein then was shock-frozen in liquid nitrogen and stored at $-80^{\circ} \mathrm{C}$. 


\title{
Cin8 wild type and Cin8 $\Delta$ tail proteins
}

\author{
Based on Düselder et al. [79] and Gerson-Gurwitz et al. [46].
}

The construct Cin8 $\Delta$ tail-TEV-GFP-6HIS was created based on pAG36 plasmid by using a PCR based approach. The Saccharomyces cerevisiae protease deficient strain was used for protein over expression [81]. Cin8 over expression and purification were carried out as described in [46]. S.cerevisiae cells expressing either the Cin8-TEV-GFP-6HIS or the Cin8 $\Delta$ tail-TEV-GFP-6HIS construct under the GAL1 promoter on a CEN plasmid were grown overnight in minimal liquid medium supplemented with $2 \%$ raffinose. For over expression, cells were added to minimal liquid medium containing $2 \%$ galactose and grown for additional $9.5 \mathrm{~h}$. Following over expression, cells were harvested, pelleted and 3X volume of lysis buffer was added (30 mM Triz/HCl, $35 \mathrm{mM}$ PIPES/KOH final $\mathrm{pH}=7.4$, 10\% glycerol, $300 \mathrm{mM}$ NaCl, 2 mM EDTA,1mM EGTA, 1 mM DTT , 1mM PMSF, 0.1 mM MgATP, $0.2 \%$ Triton X-100 and protease inhibitors). The washed pellet was ground with a mortar and pestle under liquid nitrogen. The subsequent purification of the Cin 8 variants was carried out identical to the procedures described for the wild type Eg5 protein.

\section{DK4mer construct}

Based on Thiede et al. [69].

The construction of DK4mer was based on an analysis of the transition between the motor domain and neck coiled coil in Eg5 and DmKHC. The first 345 amino acids of DmKHC were fused to Eg5 at amino acids 370. Additional constructs contained either a 6-his tag (DK4mer-his) or a green fluorescent protein (GFP)-6his cassette. Donor plasmids were kindly provided by W.O. Hancock (DmKHC) and T.M. Kapoor (FL-Eg5-GFP, BK006). We used a nested PCR approach [68] to extend the motor domain and neck linker of DmKHC with sequences providing an uninterrupted transition with selected restriction sites to a neck/stalk/tail-fragment of Eg5. The integrity of motor protein constructs was confirmed by commercial sequencing (Seqlab, Göttingen, Germany). DmKHC was amplified with a forward primer, DK1 fwd, providing an NdeI site (flanked by additional SalI and XmaI-sequences), and two reverse primers, DKrev1 and DKrev2, providing transition sequences of the Eg5 neck up to an AflI site that was generated using a silent mutation in the natural sequence (further flanking regions provide more restriction sites, such as NotI, for subcloning of fragments). The Eg 5 fragment was generated using a forward primer, EKfwd1, and two reverse primers, EKrev1 and EKrev2, providing the same restriction sites at the N-terminus and a cassette containing a sequence containing an AscI and XmaI site, followed by a 6 his box and a stop codon, followed by the cutting sites NotI, Sal, and XhoI. The AscI/XmaI site allowed us to insert a previously used GFP-6his casette from pT7-7-GFP-his. The resulting PCR fragments were initially parked in a pTOPO-XL vector (Invitrogen, Carlsbad, CA, USA) before they were subcloned into a pFastBac vector (Invitrogen, Carlsbad, CA, USA) for expression in Sf9 cells. The subsequent expression in Sf9 cells, as well as the purification, is similar to the procedures described for the wild type Eg5 protein. 


\section{Neck-linker length dependence of processive Kinesin-5 motility}

In Chapter 1, the ability of Kinesin molecular motors to move processively along a microtubule was introduced. This is one example of regulation of motility that might apply to the majority of Kinesin proteins. In the present chapter, the currently favored hypothesis, how processivity is achieved, will be examined and possible alternatives explored.

Kinesin motor proteins drive mechanical processes in cells such as long range cargo-transport or microtubule disassembly [4, 82]. Single homodimeric Kinesin-1 molecules can move processively, i.e. by taking hundreds of successive $\sim 8 \mathrm{~nm}$ steps [7], walking for several micrometers along a microtubule [28, 29]. During this motion, each step is coupled to the hydrolysis of one ATP molecule [6]. For this processive motion, the two motor heads have to be kept strictly out of phase during the chemo-mechanical stepping cycle.

Members of other Kinesin subfamilies, e.g. Kinesin-3 Kif1A/Unc104 [83, 84], Kinesin-7 CENP-E [85], Kinesin-8 Kip3p [86], or the mitotic Kinesin-5 Eg5 [61], are also able to move processively along a microtubule which indicates that the underling mechanism is conserved throughout the different Kinesin families. One possible mechanism for communication between the two heads is the gating of specific reactions in the stepping cycle by intramolecular tension transmitted by the neck linker (see Section 1.1) [34, 35, 36]. In this model, the length of the neck linker directly influences the mechanical coupling of the two heads and therefore the efficiency of the motor. Hence, a longer neck linker would disturb the inter-head communication and lead to a loss in motor coordination and a lower run length, while a shorter neck linker would tighten the coupling of the two heads resulting in an improved coordination of the two heads and a higher run length.

Interestingly, the length of the neck linker varies between the different Kinesin subfamilies. The highly processive Kinesin-1 possibly carries the shortest neck linker of 14 amino acids length while most other Kinesin have considerably longer neck linker with a length of 16 to 18 amino acids [87]. The Kinesin-5 from Xenopus laevis, Eg5, being less processive motor [88, 89], has one of the longest neck linker of 18 amino acids length [87]. These observations strongly support the described model of intramolecular tension transmitted by the neck linker.

In this chapter, we study the influence of varying neck-linker length on a Kinesin5 head/Kinesin-1 stalk chimera (see Section 1.2). Using the motor domains and the 18 amino acids long neck linker of the Kinesin-5 Eg5 enabled us to explore the whole range of native neck linker. By truncating the Eg5 sequence, we generated neck linker as short as, or even shorter than Kinesin-1, while the complete sequence 


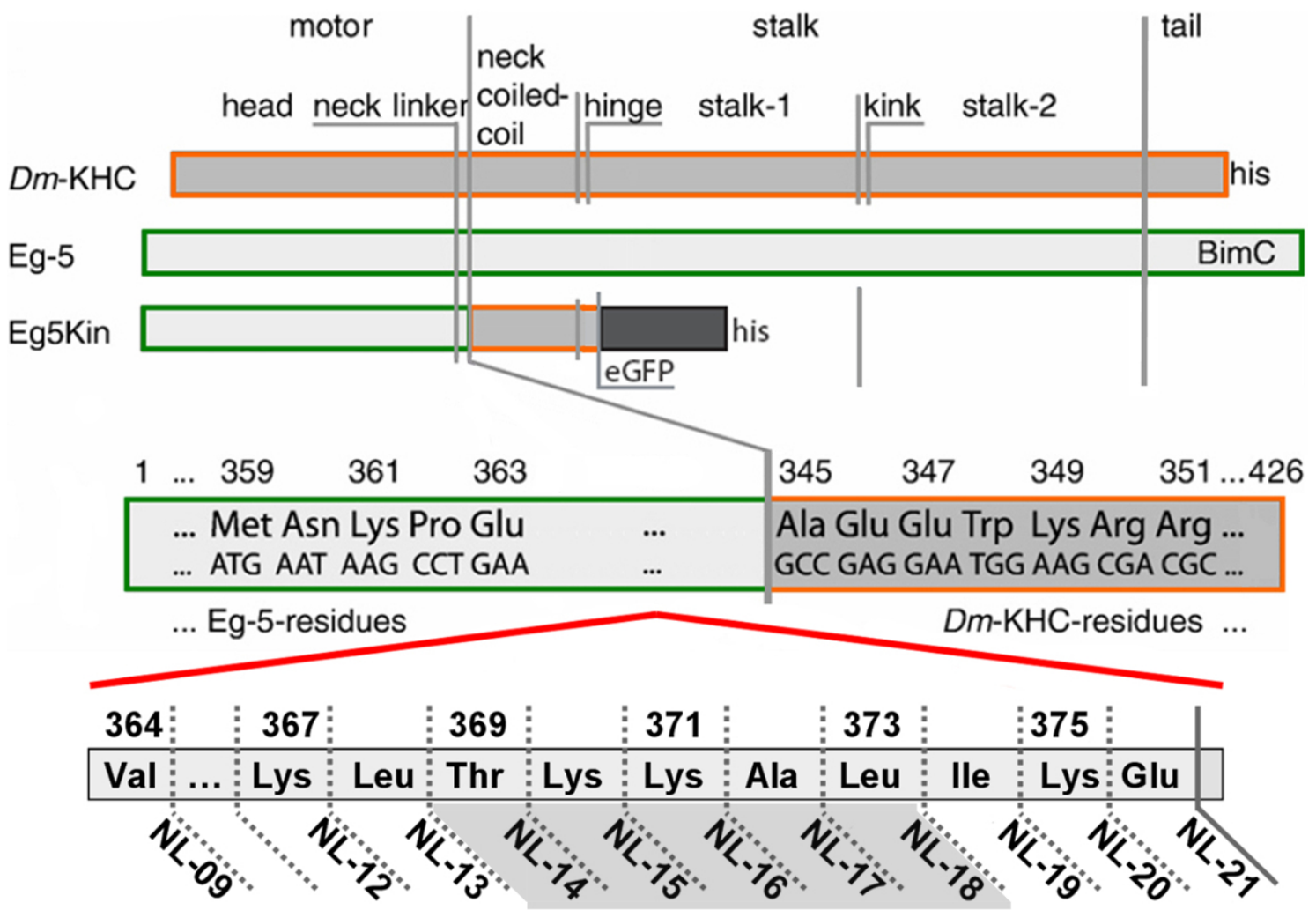

Figure 3.1: Schematic representation of Eg5Kin-NL constructs

Sequence of the chimeric construct Eg5Kin. The motor domain and part of the neck-linker sequence of Eg5 (light gray; residues 1 - 364 up to 1 - 376 for NL-09 to NL-21, respectively) were fused with the (truncated) stalk of DmKHC (dark gray; DmKHC residues 345 - 426). The numbering refers to the amino acid numbering in the respective wild type motor sequences. Figure adapted from Düselder et al. [79].

provided us with one of the longest neck linker to be found in the Kinesin family. Thus, we directly investigated whether the simple predictions of a tighter mechanical coupling of both head groups results in a higher processivity.

To perform this study, we built twelve different motor constructs ${ }^{1}$. In these constructs, we systematically varied the length of the neck linker that connects the Kinesin-5 heads with the Kinesin-1 stalk. In contrast to other studies where artificial randomly generated sequences were used for the neck linker modification [39, 43, 90, 91], we used the native Kinesin-5 sequence which includes a neck linker with the length of 18 amino acids [87] as a template (Figure 3.1). We successively removed single amino acids from the sequence to produce the constructs NL-18 (no amino acids deleted) down to NL-12 (6 amino acids deleted) and one even shorter construct with a neck linker length of 9 amino acids (NL-09).

To elongate the neck linker beyond the range of the native protein, we continuously added up to three further amino acids from the putative neck coiled-coil of Eg5 (NL-19 to NL-21). While in the native protein this sequence marks the onset of coiled-coil formation, a coiled-coil probability analysis ${ }^{2}$ for our chimeric proteins showed no sign of increase in coiled coil formation probability when extending the

\footnotetext{
${ }^{1}$ Protein cloning, expression and purification was done by Kerstin von Roden, Charlotte Willms, and Dieter Klopfenstein.

${ }^{2}$ Coiled-coil probability prediction was done by Stefan Lakämper.
} 

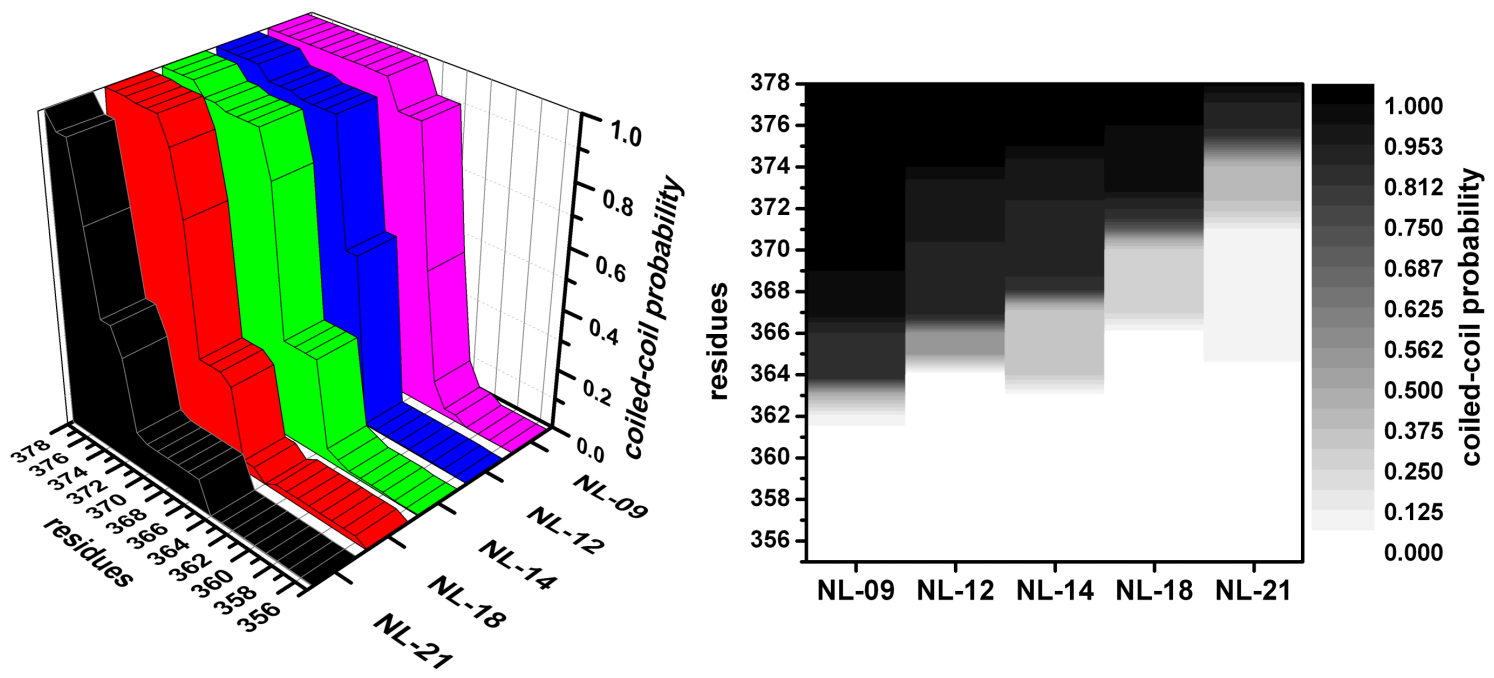

Figure 3.2: Coiled-coil probability analysis

Coiled-coil probability at different residues for exemplary constructs. The onset of the coiled-coil formation changes according to neck-linker length variation. Start of the wild type neck-linker sequence is residue 355. Prediction of coiled-coil formation probability was done using NCOILS by R.B. Russell and A.N. Lupas based on [93].

Eg5 sequence in the Kinesin-1 background (Figure 3.2). To additionally check for any effects introduced by this elongation, we built one more construct in which we extended the neck linker by adding three prolines after the native neck linker sequence (NL-21P, not shown in Figure 3.1). These prolines were too short to form a poly-proline structure and therefore ideal as a neutral extension for the neck linker [92]. As a control whether all constructs are dimeric proteins, a SDS-PAGE gel ${ }^{3}$ was made. Comparison of the molecular weight of the constructs proved that all proteins are dimers (Figure 3.3).

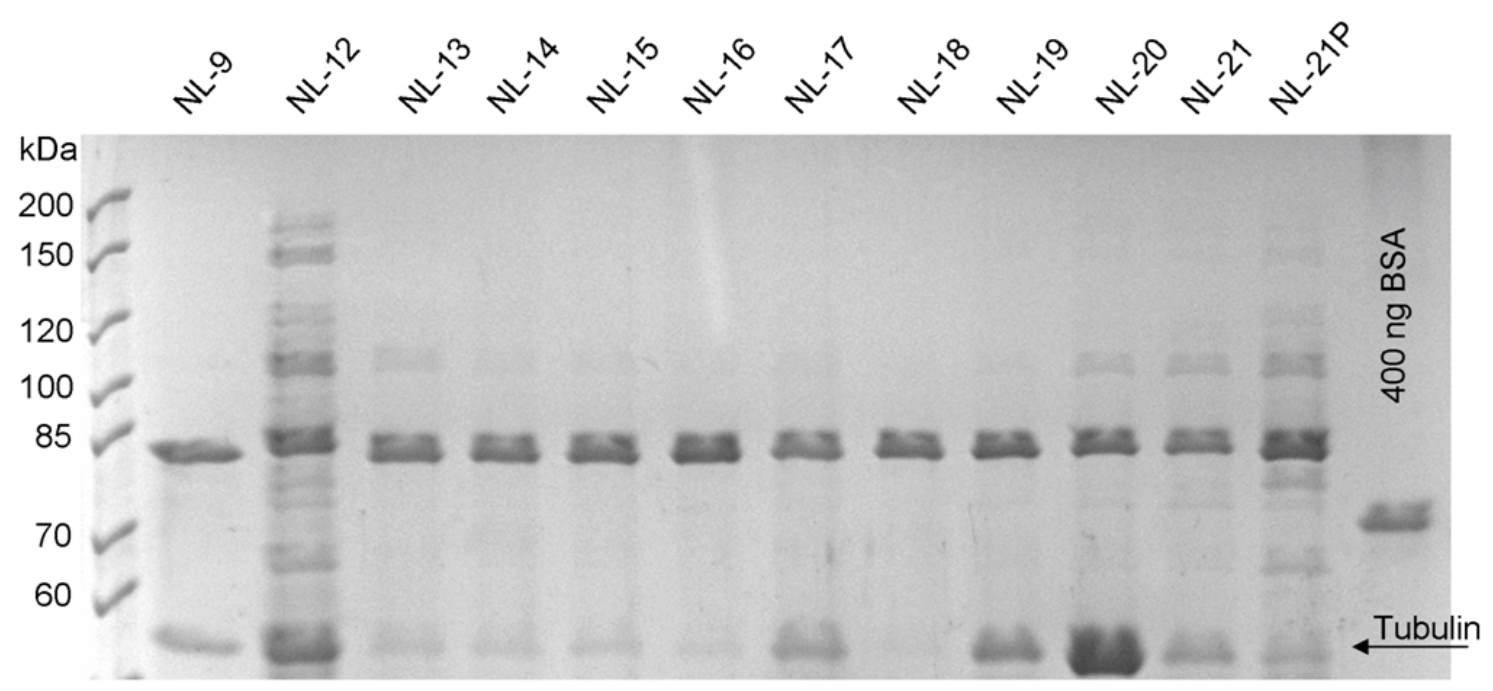

Figure 3.3: SDS-PAGE gel

Coomassie-stained SDS-PAGE gel (4\% stacking gel and 10\% separation gel) of all neck-linker constructs. Concentrations were chosen to result in an equal amount of protein for each construct ( $400 \mathrm{ng}$ ). $400 \mathrm{ng}$ of BSA shown for comparison. Figure adapted from Düselder et al. [79].

\footnotetext{
${ }^{3}$ Fabrication and analysis of the SDS gel was done by Kerstin von Roden and Charlotte Willms.
} 


\subsection{Influence of neck-linker length on velocity}

To test the viability of our motor constructs and measure the velocity produced by motors acting collectively, we performed surface-gliding assays ${ }^{4}$ (see Section 2.3) with all twelve constructs. We found that all constructs were active motor proteins. They were capable of sliding microtubules along a surface with nearly identical velocities of $\sim 67 \mathrm{~nm} / \mathrm{s}$ on average (Figure 3.4 and Table 3.1 on page 43 ). The only exception was the shortest construct, NL-09, which showed a clearly diminished velocity of $16 \pm 6 \mathrm{~nm} / \mathrm{s}$ (mean $\pm \mathrm{SD}$ ). The short neck linker in the NL-09 construct could already be below the minimum length that is required to bridge two adjacent motor binding sites on the microtubule. While the other constructs might be processive motors, it is possible that NL-09 proteins were only capable of performing single power-strokes before unbinding which could result in a decreased average velocity.

To measure the velocity of individual motors, we used two different experimental approaches. One approach was the use of single-bead optical trapping assays (see Section 2.3). All neck-linker constructs with a neck-linker length of 12 or longer were able to move the beads relative to the trap with the same average initial velocity of $\sim 64 \mathrm{~nm} / \mathrm{s}$ (figure $3.4 \mathrm{~A}$ and Table 3.1 on page 43 ). This is in good agreement with the ensemble averaged velocity of multiple motors that was measured in the surface-gliding assay. However, no active motion for the shortest construct NL-09 could be detected in the single-bead assay. This indicates that NL-09 might be an active motor that can bind to microtubules and perform a single step, but is non-processive and therefore unable to take multiple successive steps.

The second experimental approach was to use single-molecule fluorescence assays $^{5}$ (see Section 2.3). We found that all constructs, with the exception of NL-09, were processive motor proteins that moved along single, surface-immobilized microtubules (Figure 3.5). Their velocities were largely similar with an average value of $\sim 116 \mathrm{~nm} / \mathrm{s}$ (Figure $3.4 \mathrm{~A}$ and Table 3.1 on page 43). This value is slightly larger than the results from the other assays. This can be explained if we look at the loaded versus unloaded situation in the different assays. In the surface gliding as well as the optical-trapping assay, the motor had to work against a load. Surface-gliding assays can always suffer from attachment artifacts or contamination by defective motors. In optical trapping assays the motor has to pull the bead and work against the force induced by the trap. This leads to reduced velocities in comparison with the completely load free setup in the single-molecule fluorescence assays [7]. In the single-molecule assays as well as in the previous experiments, the NL-09 construct behaved differently. As was expected from the trapping assay, no processive motion could be detected for NL-09 in the single-molecule fluorescence assay. This is another strong indicator that the 9 amino acids long neck linker in NL-09 is not long enough to reach from one binding site on the microtubule lattice to the next. As assumed from the surface-gliding assays, this prevented the motor from processively walking along a microtubule, forcing it to move with single power strokes.

\footnotetext{
${ }^{4}$ Surface-gliding assays performed and analyzed by Christina Thiede.

${ }^{5}$ Single-molecule fluorescence assays performed and analyzed by Christina Thiede.
} 
A

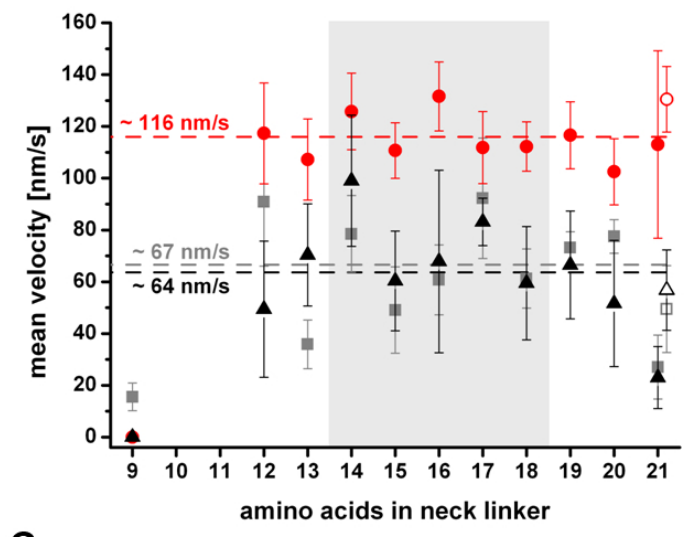

C

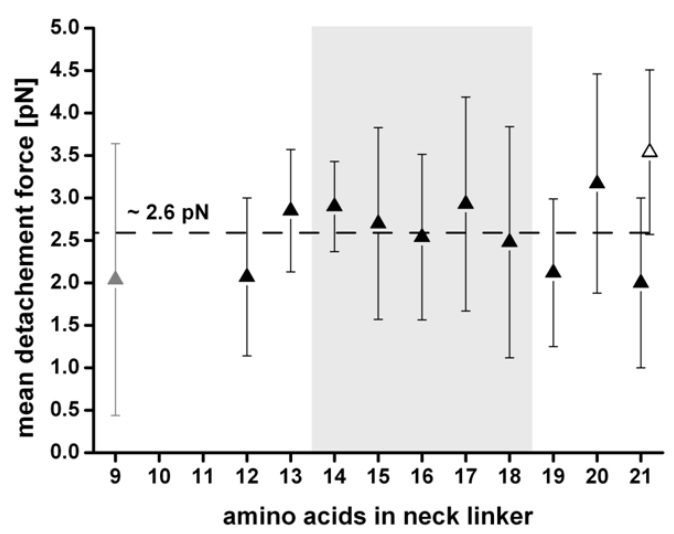

B

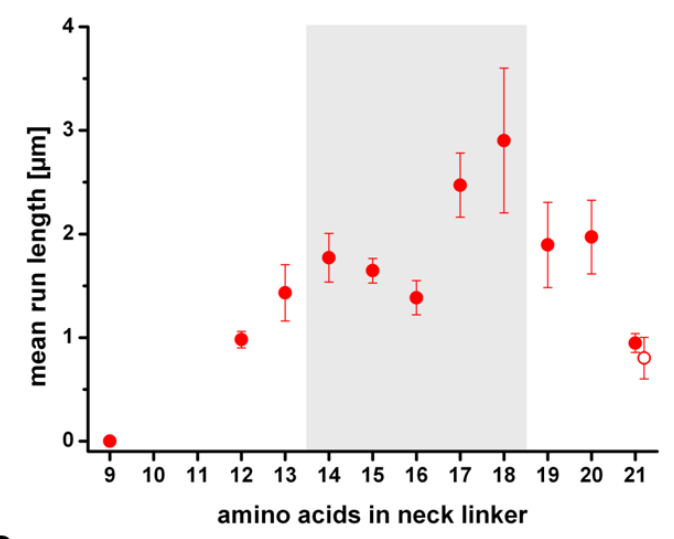

D

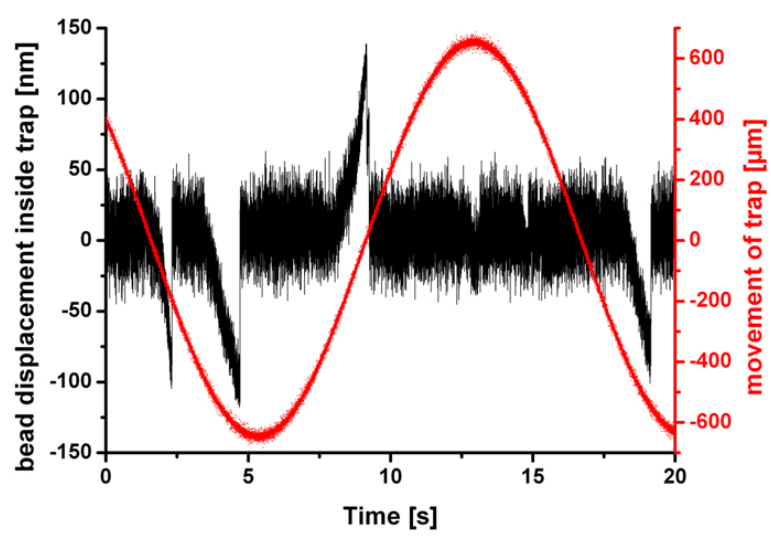

Figure 3.4: Dependence of motile properties on neck-linker length

A Velocities for all neck-linker constructs (mean $\pm \mathrm{SD}$ ). Red circles: single-molecule fluorescence assay; black triangles: optical trapping assay; gray squares: surface-gliding assay. The average values for the different data sets are indicated by the dashed lines. B Processive run lengths for all neck-linker constructs obtained with single-molecule fluorescence assays (mean $\pm \mathrm{SD}$ ). No processive motion could be detected for the NL-09 construct. C Detachment forces for all constructs in optical trapping assays (mean $\pm \mathrm{SD}$ ). D Force measurement for the non-processive NL-09 construct. The microscope stage was moved in a sinusoidal fashion (frequency $=0.06 \mathrm{~Hz}$; amplitude $=660 \mathrm{~nm}$ ) while the trap position was fixed.

The range of natural length of the neck-linker of Kinesin-1 and Kinesin-5 (14 - 18 amino acids) is indicated by gray shading. Open symbols: NL-21P. Figure adapted from Düselder et al. [79].

Our experiments so far showed that the variation of the length of the neck linker has no strong influence on the velocity of a Kinesin-5 head/Kinesin-1 stalk chimera. These findings are in contradiction to previous reports.

Shastry and Hancock investigated the effect of changing the neck-linker length on a Kinesin-1 and an artificially homodimeric Kinesin-2 construct [91]. They found a drastic decrease of velocity for the Kinesin-1 motor when shortening as well as extending the native neck linker while their Kinesin-2 construct was, similar to our study, largely unaffected [91].

Clancy et al. found a distinct decrease in unloaded velocity for a Kinesin-1 construct when elongating the neck linker to a length of 20 amino acids [43]. They provided evidence that a drastic extension of the neck linker leads to a decrease in motor coordination which results in increased back-stepping events [43]. The discrepancy between our results and that of Clancy et al. may be explained by the fact that Kinesin- 1 proteins have an intrinsically higher velocity than Kinesin- 5 motors 

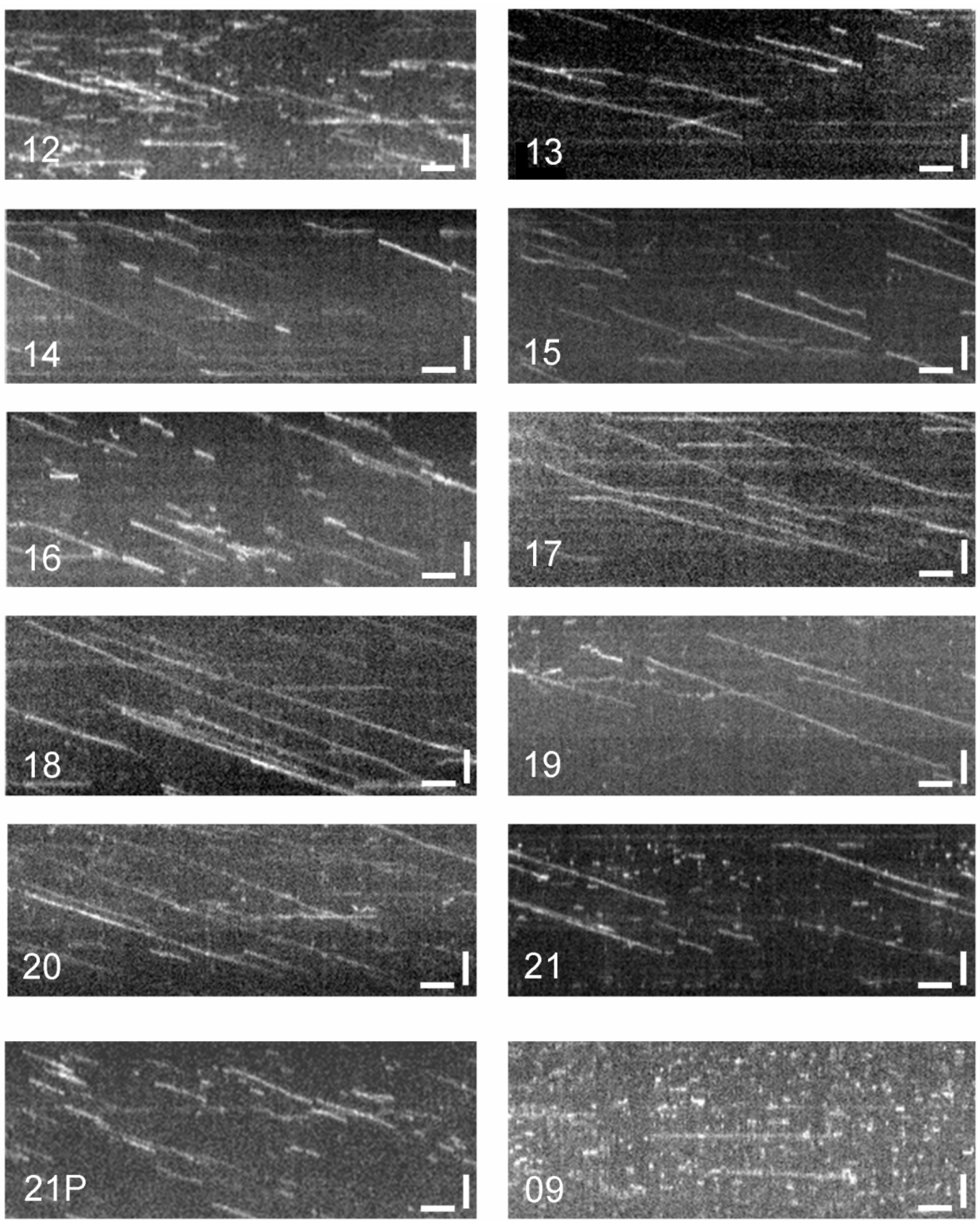

Figure 3.5: Kymographs of all constructs

Kymographs of GFP-labeled neck-linker constructs moving on surface-immobilized microtubules. The designation of the used construct is given in the lower-left corner of the respective kymograph. Note the change in run length depending on the neck-linker length. Scale bars: horizontal $10 \mathrm{~s}$; vertical $3 \mu \mathrm{m}$. Figure adapted from Düselder et al. [79]. 
and are therefore most likely posses a different catalytic cycle. Thus, the loss of coupling efficiency might affect Kinesin-1 related proteins differently than Kinesin- 5 constructs.

In another study, Yildiz et al. observed a decrease in the single-molecule velocity that was measured for Kinesin-1 constructs with a neck linker that was extended by the addition of polyproline inserts of various length [90]. The authors could not measure a change in the ATP turnover rate $k_{\text {cat,ATP }}$ and therefore concluded that the coupling between stepping and ATP hydrolysis was diminished [90]. This could be due to an increase in flexibility of the neck linker which is supported by the fact that they were able to "rescue" the motor velocity by applying an assisting load. In our assays, there was no detectable change or trend in the observed velocities of the constructs. This suggests that the neck-linker length neither influences the rate at which the Kinesin-5 motor domains hydrolyze ATP nor the coupling efficiency between ATP hydrolysis and stepping during the interaction of the Kinesin-5 motor domains with the microtubule.

\section{Force generation is independent of neck-linker length}

In addition to the velocity, the detachment force of a motor protein can be measured in a single-bead assay. The maximum displacement from the trap in the experimental results (Figure 3.6) can be converted to a force value if the stiffness of the trap is known (for details see Section 2.1). From the measurements of all twelve necklinker constructs, we calculated the detachment force of the motors. We found no significant variation between the different constructs (Figure 3.4 C and Table 3.1 on page 43). All motors detached from the microtubules in a Kinesin-5 typical manner prior to stalling [10]. The mean detachment force for the whole set of proteins was $\sim 2.6 \mathrm{pN}$. This is considerably lower than for Kinesin-1, which has an average stall force of $\sim 7 \mathrm{pN}$ but in good agreement with previously measured values for wild type Kinesin-5 Eg5 [10] and Kinesin-5 head/Kinesin-1 stalk chimeras [68]. Though the single-molecule assays showed that the NL-09 construct is a non-processive motor, we were still able to obtain a force value for this motor. The surface-gliding assays offered proof that NL-09 is an active motor. Considering this fact, we changed the position of the microscope stage in a sinusiodal movement while keeping the trap position constant. In this way, the motor-covered beads were dragged along a surface-immobilized microtubule (Figure $3.4 \mathrm{D}$ ). The binding of the motors to the microtubule made the bead follow the movement of the stage. Since the position of the trap was fixed, this led to a built up in force. This force was high enough to reach the detachment force of NL-09, which was similar to that of the other constructs (Figure $3.4 \mathrm{C}$ and Table 3.1 on page 43 ).

These results demonstrate that the variation of the length of the neck linker of the motor has no immediate effect on its force generation. Yildiz et al. showed that they could restore the diminished velocities of their neck linker extended construct by applying a constant assisting load [90]. Since we only measured in a constant position instead of constant force geometry, the results might not be directly comparable. 

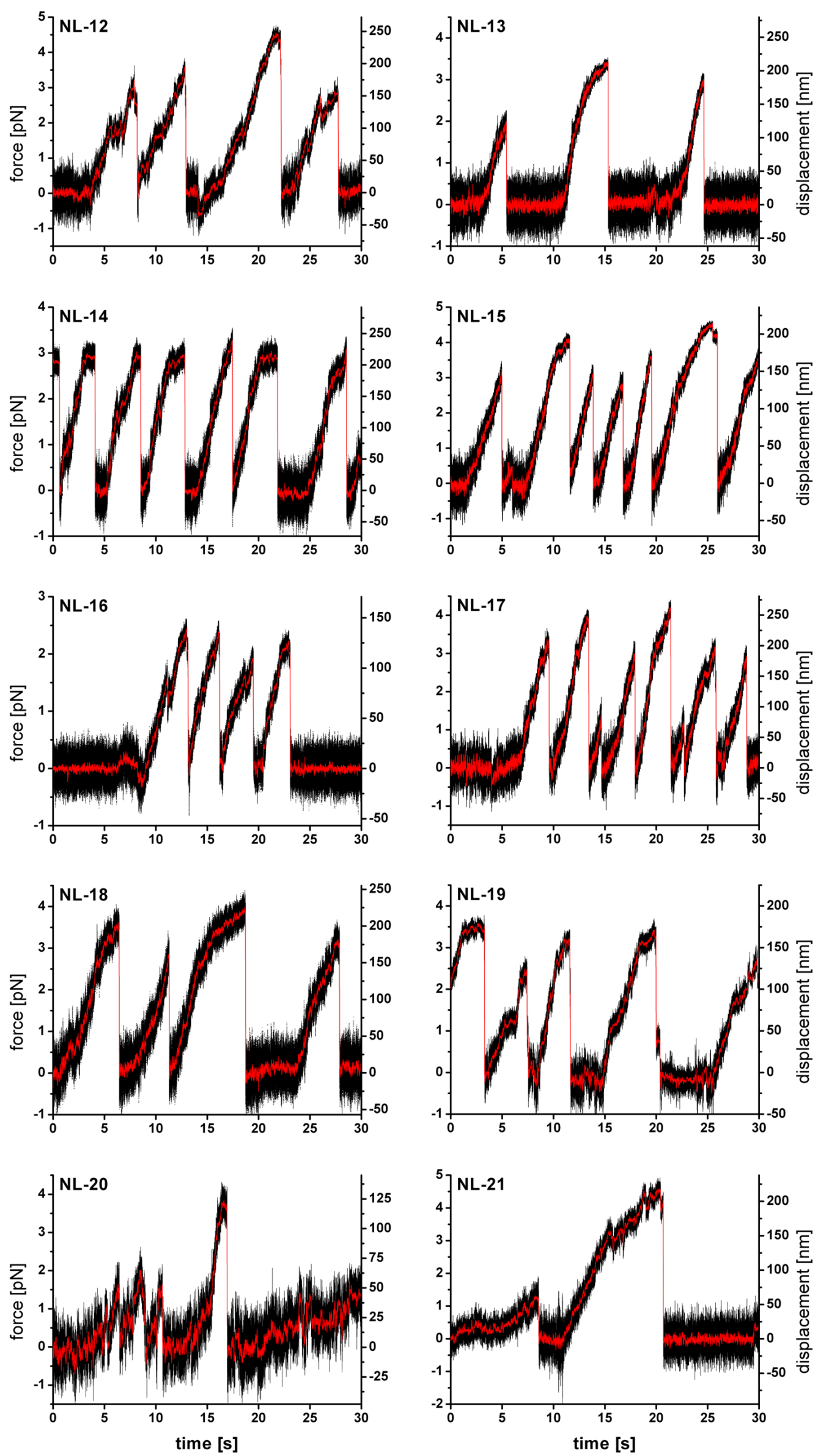


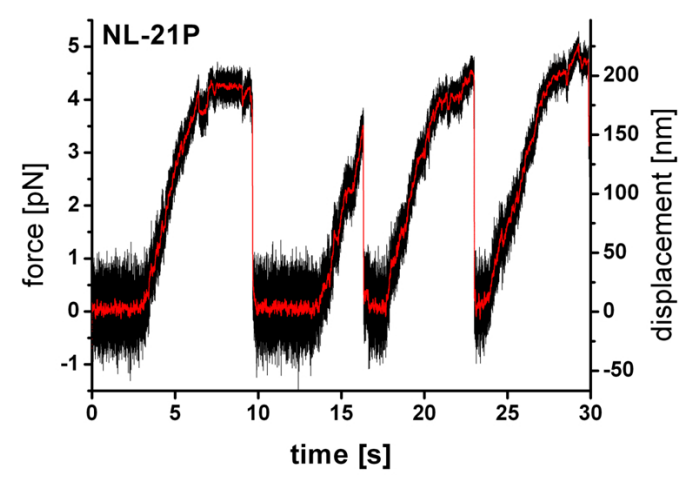

Figure 3.6: Exemplary trap results of all constructs

Exemplary trap traces for all constructs except NL-09. The slope of a given trace is used to determine the initial velocity while the peak of an excursion from the trap corresponds to the motors' unbinding force. No stalling can be observed. The trap stiffness ranges from $\sim 0.013$ to $\sim 0.022 \mathrm{pN} / \mathrm{nm}$. Raw data shown in black, 0.03 s sliding window median filtered data in red.

All of our constructs show the same behavior in our trapping experiments and the velocities are constant over all measured neck-linker lengths, it is unlikely that we would observe a similar "rescuing" of the motor's motility. Clancy et al. saw a decrease in stall force in their measurements for a Kinesin-1 construct with elongated neck linker. They found evidence that this is the result of an increase in back stepping events [43]. We, on the other hand, were unable to see any increase in back stepping. We conclude that the force application on the motors due to the trap does not influence its motility. More specifically, the effects of neck-linker length modification on the force generation of our constructs are negligible.

\section{Step size is unchanged by variation of neck-linker length}

A further analysis of the trapping data allowed us to investigate the effects of the altered neck linker on the step size of the motor. It is possible, given a sufficient elongation of the neck linker, that the motor proteins take "double-steps". A motor could be able to skip one binding site along the microtubule, resulting in a $16 \mathrm{~nm}$ step. Another possibility is that the change in communication between the two heads and the connected loss of efficiency in the chemo-mechanical stepping cycle could lead to an increased probability of back-stepping events (as was previously reported [43]). To calculate the step size of the different neck-linker constructs, we used a step-finding algorithm as was proposed by Kerssemakers et al. [94]. Exemplary results of this step size analysis are shown in Figure 3.7.

The obtained step sizes were attenuated by the compliance of the motor bead linkage and thus smaller than $8 \mathrm{~nm}$. To estimate the stiffness of this linkage $\mathrm{k}_{\text {motor }}$, we analyzed the variance of the displacement signal from the trapping assays and calculated a stiffness of $0.1 \mathrm{pN} / \mathrm{nm}$. To obtain the motor's actual step size $\Delta_{\text {motor }}$ we used the correction $\Delta_{\text {motor }}=\Delta_{\text {bead }} \cdot\left(\mathrm{k}_{\text {trap }}+\mathrm{k}_{\text {motor }}\right) / \mathrm{k}_{\text {motor }}$. The average of the compliance-corrected step sizes given in Figure $3.7 \mathrm{D}$ is $\sim 8.7 \mathrm{~nm}$ which is in good agreement with the distance between two binding sites along the microtubule lattice [7]. Furthermore, we saw no increase in back-stepping events, which would result in a negative step size, in our experiments. 
A

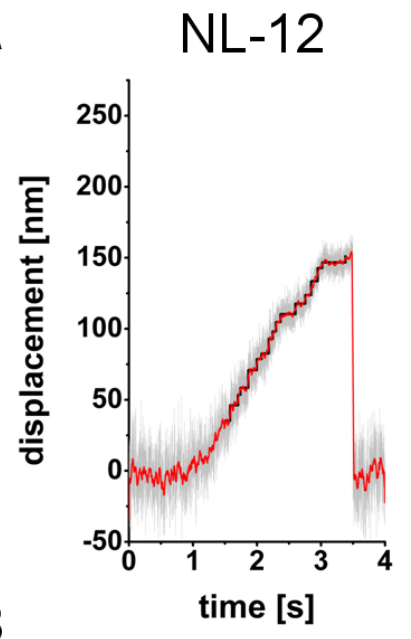

B

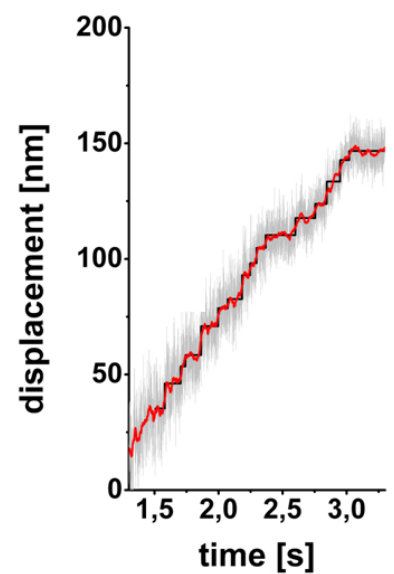

$\mathrm{NL}-14$
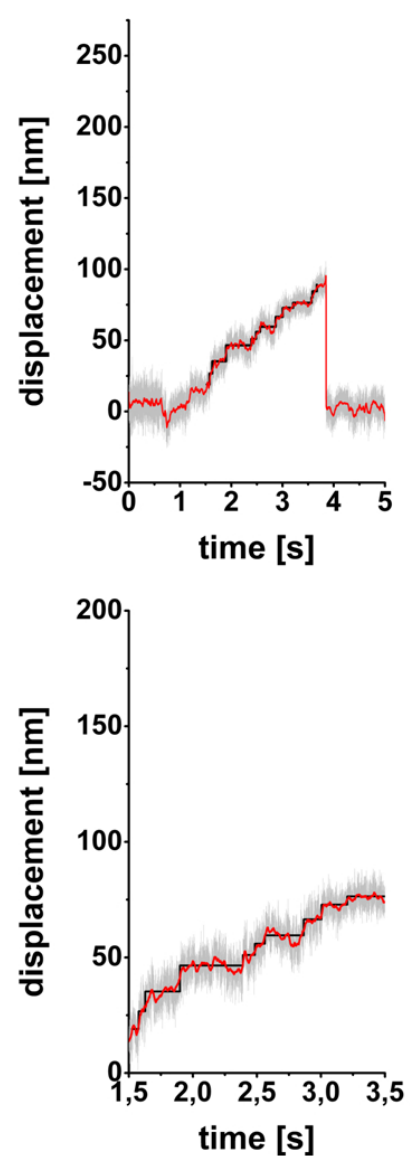

$\mathrm{NL}-21$
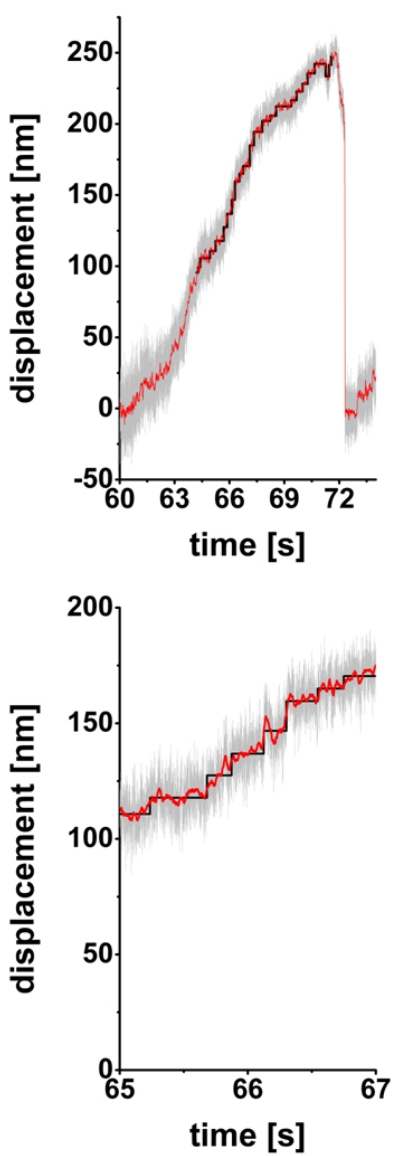

C

\begin{tabular}{|c|c|c|c|c|c|c|c|c|c|c|c|}
\hline NL length [aa] & 12 & 13 & 14 & 15 & 16 & 17 & 18 & 19 & 20 & 21 & $21 \mathrm{P}$ \\
\hline step size [nm] & $\begin{array}{c}6.1 \\
\pm 0.5\end{array}$ & $\begin{array}{c}6.9 \\
\pm 0.3\end{array}$ & $\begin{array}{c}5.6 \\
\pm 0.4\end{array}$ & $\begin{array}{c}8.0 \\
\pm 0.3\end{array}$ & $\begin{array}{c}8.2 \\
\pm 0.5\end{array}$ & $\begin{array}{c}9.3 \\
\pm 0.3\end{array}$ & $\begin{array}{c}6.7 \\
\pm 0.3\end{array}$ & $\begin{array}{c}7.7 \\
\pm 0.3\end{array}$ & $\begin{array}{c}5.5 \\
\pm 0.1\end{array}$ & $\begin{array}{c}7.7 \\
\pm 0.4\end{array}$ & $\begin{array}{c}6.9 \\
\pm 0.3\end{array}$ \\
\hline $\mathrm{N}$ & 104 & 84 & 141 & 57 & 72 & 54 & 47 & 62 & 128 & 68 & 103 \\
\hline
\end{tabular}

Figure 3.7: Motor step size analysis

Exemplary results for three different constructs are shown. A Bead-displacement time series from the optical trapping assay (raw data in gray, $0.03 \mathrm{~s}$ median filtered data in red). The results of a step size analysis are shown in black. B Magnification of the data fitted by the step-finding algorithm in A. C Summary of the uncorrected step size for all NL-constructs (mean \pm SEM). N: Number of analyzed steps indicated. Figure adapted from Düselder et al. [79].

Thus we ruled out any of the aforementioned possible influences of the neck linker extension on our constructs. Neither an increase in back-stepping events nor any double- or side-steps resulted from the variation of the neck linker. 


\section{Varying neck-linker length affects motor run length}

The last characteristic of the motor constructs we investigated was the effect of modified neck-linker length on the run length of the motor. From the kymographs ${ }^{6}$ (Figure 3.5), two things can immediately be seen: firstly, all proteins were capable of processive motion, with the exception of the construct with the shortest neck linker, NL-09. This is consistent with and even goes beyond the recently reported results that Kinesin-1/2 and Kinesin-7 constructs with neck linkers as short as 13 amino acids were able to support processive motion [43]. The shortest neck linker in wild type molecular motors can be found in Kinesin-1 motors with 14 amino acids [87]. With this study, we could further support the notion that this might not necessarily present an absolute lower boundary. In our experiments, proteins with neck linker as short as 12 amino acids were still capable of processive motion. The neck linker in the NL-09 construct, however, was probably below the limiting neck linker length. In this motor, the neck linker might be unable to bridge the distance between two motor binding sites on a microtubule.

Secondly, the run length did indeed change with altered neck linker length. A closer analysis revealed that there are several differences between the constructs. While the NL-09 motor, as a non-processive motor, showed no measurable run length, the constructs with a longer neck linker up to NL-14 showed a gradual increase in run length (Figure $3.4 \mathrm{~B}$ ). Further elongation to 15 and 16 amino acids led to a decrease in run length as was expected from the intramolecular tension model. However, when approaching the native neck-linker length of 18 amino acids for Kinesin-5, the motor's run length drastically increased to reach a maximum run length of $\sim 3 \mu \mathrm{m}$ (Figure $3.4 \mathrm{~B}$ ). Extending the neck linker beyond the native length of 18 amino acids led again to a lowered run length. Since an arbitrary long neck linker would at one point certainly disrupt the coordination of the two motor heads, this is to be expected.

To check whether multimerization of the proteins could have influenced these results, we performed a bleaching step analysis ${ }^{7}$ (Figure 3.8). For all constructs, we observed a two step bleaching behavior. The formation of protein aggregates beyond the expected dimers is therefore highly unlikely.

These findings contradict the previously discussed intramolecular tension model. Notwithstanding, we see a small peak in run length for 14 amino acids corresponding to the native neck-linker length of Kinesin-1, a motor with a reportedly high run length $[7,95]$. The maximum run length is achieved for the neck linker-length native to the studied Kineisn-5 protein. The simple model in which a shorter neck linker equals a tighter mechanical coupling of the two heads and therefore a more efficient chemo-mechanical cycle of the motor cannot explain these findings. While the local maximum value for the run length at a neck-linker length of 14 amino acids could be a consequence of the tighter mechanical coupling, the even higher run length at the native neck-linker sequence points toward another, more determining factor. Clancy et al. reported an increase in run length for constructs with neck-linker

\footnotetext{
${ }^{6}$ Single-molecule fluorescence assays performed and analyzed by Christina Thiede.

${ }^{7}$ Photo-bleaching experiments performed and analyzed by Christina Thiede.
} 
A
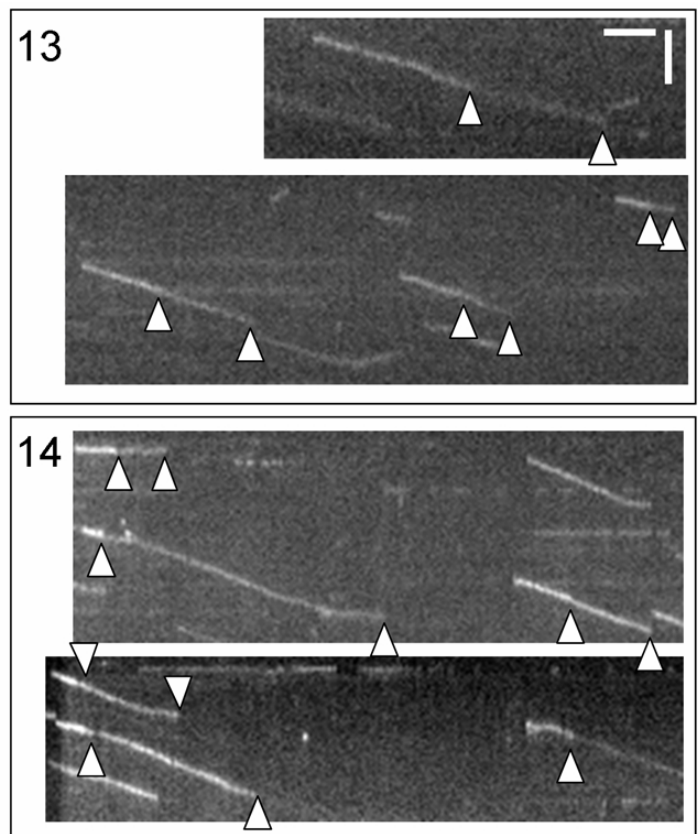

15

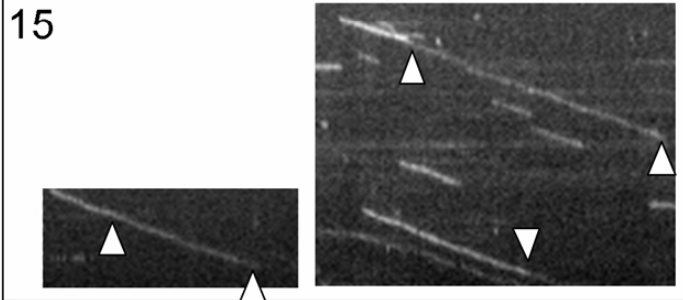

21
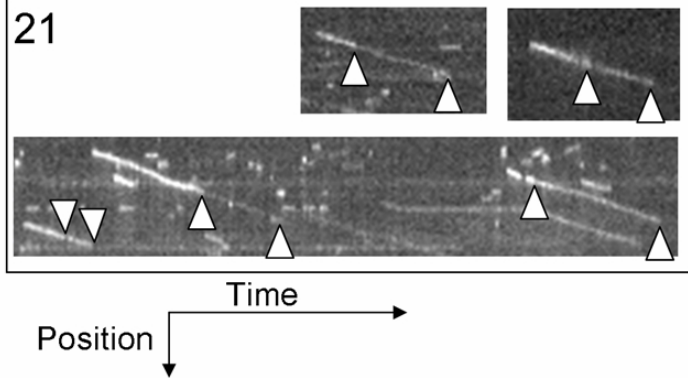
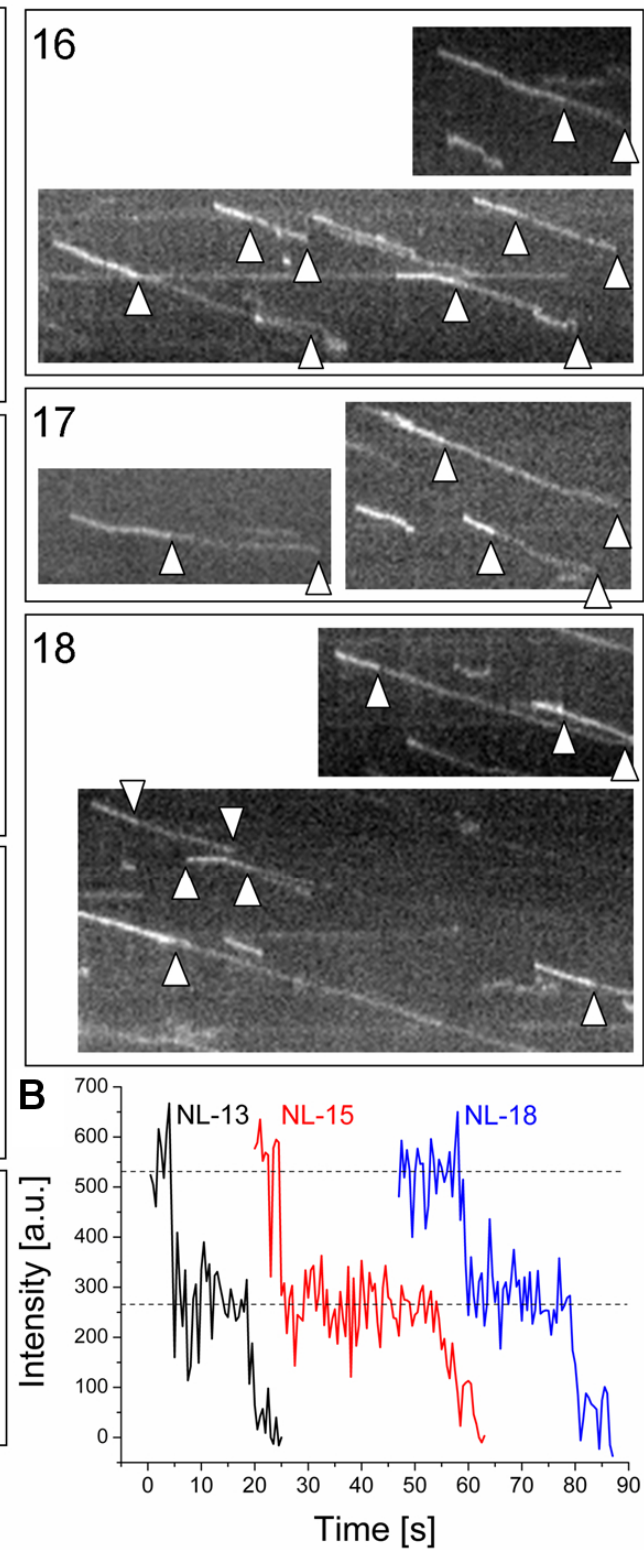

Figure 3.8: Photo-bleaching analysis for neck linker constructs

A Kymographs of GFP-labeled neck-linker constructs moving on surface-immobilized microtubules. The arrowheads mark photo-bleaching events of moving motor constructs. Scale bar: horizontal $10 \mathrm{~s}$, vertical $3 \mu \mathrm{m}$ B Exemplary bleaching curves for three different neck-linker constructs. A two steps bleaching process is apparent. Figure adapted from Düselder et al. [79].

length beyond 14 amino acids [43]. One possible explanation is that, rather than the mechanical properties, it is the sterical orientation of the neck linker during the motor's stepping cycle that may be the major influencing factor for the motile properties of a molecular motor [43]. 


\section{\begin{tabular}{l|l} 
Summary & 3.5
\end{tabular}}

Given the experimental evidence (Table 3.1) that the variation of the neck-linker length affects processivity, it remains to discuss the consequences for the simple gating models for Eg5 in particular or for all Kinesins in general. Two physically different gating scenarios seem possible. Either (i) neck-linker orientation is the deciding parameter, and inter-head tension is not important for the processivity, or (ii) each Kinesin family has a particularly adapted neck-linker length and/or specific interactions between the native neck linker and the rest of the motor together optimize the transmission of tension and the gating and stepping mechanism.

\begin{tabular}{lccc|cccc|cc} 
& \multicolumn{3}{c|}{ SBMA } & \multicolumn{5}{|c|}{ SMFA } & \multicolumn{2}{c}{ SGA } \\
\cline { 2 - 10 } Protein & $\begin{array}{c}\text { Velocity } \\
(\mathrm{nm} / \mathrm{s})\end{array}$ & $\begin{array}{c}\text { Force } \\
(\mathrm{pN})\end{array}$ & $\mathrm{N}$ & $\begin{array}{c}\text { Velocity } \\
(\mathrm{nm} / \mathrm{s})\end{array}$ & $\begin{array}{c}\text { Run length } \\
(\mu \mathrm{m})\end{array}$ & $\mathrm{N}$ & $\begin{array}{c}\mathrm{C}_{\text {TIRF }} \\
(\mathrm{nM})\end{array}$ & $\begin{array}{c}\text { Velocity } \\
(\mathrm{nm} / \mathrm{s})\end{array}$ & $\mathrm{N}$ \\
\hline \hline NL-09 & $0 \pm 0^{a}$ & $2.0 \pm 1.6$ & 33 & $0 \pm 0^{a}$ & $0 \pm 0^{a}$ & 80 & 178 & $16 \pm 6$ & 41 \\
NL-12 & $70 \pm 20$ & $2.1 \pm 0.9$ & 109 & $117 \pm 20$ & $0.9 \pm 0.1$ & 104 & 157 & $19 \pm 25$ & 37 \\
NL-13 & $99 \pm 25$ & $2.9 \pm 0.7$ & 100 & $107 \pm 16$ & $1.4 \pm 0.3$ & 102 & 105 & $36 \pm 9$ & 29 \\
NL-14 & $60 \pm 19$ & $2.9 \pm 0.5$ & 50 & $125 \pm 15$ & $1.8 \pm 0.2$ & 124 & 131 & $78 \pm 15$ & 28 \\
NL-15 & $68 \pm 35$ & $2.7 \pm 1.1$ & 98 & $111 \pm 11$ & $1.7 \pm 0.1$ & 130 & 52 & $49 \pm 17$ & 20 \\
NL-16 & $83 \pm 9$ & $2.5 \pm 0.9$ & 35 & $132 \pm 13$ & $1.4 \pm 0.2$ & 269 & 189 & $61 \pm 14$ & 17 \\
NL-17 & $60 \pm 22$ & $2.9 \pm 1.3$ & 63 & $112 \pm 14$ & $2.5 \pm 0.3$ & 162 & 53 & $92 \pm 23$ & 23 \\
NL-18 & $59 \pm 22$ & $2.5 \pm 1.4$ & 68 & $112 \pm 10$ & $2.9 \pm 0.7$ & 145 & 316 & $61 \pm 11$ & 20 \\
NL-19 & $66 \pm 21$ & $2.1 \pm 0.9$ & 321 & $117 \pm 13$ & $1.8 \pm 0.4$ & 106 & 158 & $37 \pm 6$ & 34 \\
NL-20 & $52 \pm 24$ & $3.2 \pm 1.3$ & 109 & $103 \pm 13$ & $1.9 \pm 0.3$ & 98 & 132 & $77 \pm 7$ & 30 \\
NL-21 & $23 \pm 12$ & $2.0 \pm 1.0$ & 67 & $113 \pm 36$ & $0.8 \pm 0.2$ & 106 & 317 & $27 \pm 13$ & 32 \\
NL-21P & $57 \pm 16$ & $3.5 \pm 1.0$ & 134 & $131 \pm 13$ & $0.9 \pm 0.1$ & 128 & 95 & $49 \pm 18$ & 39 \\
\hline
\end{tabular}

Table 3.1: Summary of the three measurements for all NL constructs

SBMA: single-bead motility assays; SMFA: single-molecule fluorescence assay;

SGA: surface-gliding assay; all values: mean \pm standard deviation;

$\mathrm{C}_{\mathrm{TIRF}}$ : protein concentration in single-molecule fluorescence assay;

$\mathrm{N}$ : number of beads, single motors, and microtubules tracked, respectively;

${ }^{a}$ : no processive motility detected.

Table adapted from Düselder et al. [79].

Gating scenario (ii), on one hand, seems to be supported by the finding that replacement and addition of single amino acids in several Kinesins alter processivity $[33,91]$. We did not see such strong effects. Furthermore, a comparison between motors with different extension sequences but equal length, NL-21 and NL-21P, indicates that it is unlikely that the particular sequence of the extension played a dominating role in determining processive run lengths.

On the other hand, gating scenario (i) is supported by an increase of processivity with strongly extended neck linkers for Kinesin-1 constructs [43]. This result was interpreted to be caused by a two-head-bound waiting state with a smaller probability of total release. Since this mechanism also entails lower velocities, it is likely that this was not exactly the case for our intermediate-length Kinesin- 5 constructs. In essence, head-head communication via neck-linker orientation - and not tension 
- is consistent with our results. Interestingly, two studies, reporting a structurally unusual neck-linker orientation [96] and two-state neck-linker orientation kinetics [97] further argue for this possibility, particularly for Kinesin-5.

In summary, a simple mechanistic model generally applying to all processive Kinesins - equating a shorter neck-linker length directly to more tension and to more efficient gating and thereby to more processivity, with one universal optimal number for the neck-linker length - might not exist. In view of the broad variations in function, regulation, and oligomerization properties of Kinesins, it would indeed be surprising if a single parameter, namely neck-linker length, could determine performance. Coordination between the heads or gating, however, is a necessity for processive Kinesins. Some of the existing data, including ours, now point to the interesting possibility that the orientation of the neck linker plays a crucial role, rather than merely length and tension. This opens up the option that different Kinesins could achieve optimal head-head communication with different neck-linker lengths. The native neck-linker length and sequence might be optimized, in each case, for both effective communication and processivity, as well as other specific functions such as regulation. 


\section{Directionality of single Kinesin-5 Cin8 molecules is mediated by the tail domains}

After studying the possible mechanisms that enable processive motion of Kinesin molecular motors in Chapter 3, we will look at a more specific regulatory process. For three decades, it was believed that processive motion of Kinesin motors is always directed toward the plus end of a microtubule. Recently, however, it was shown that some members of the Kinesin-5 subfamily of motor proteins are capable of bidirectional motion. How this bidirectionality is regulated will be covered in the present chapter.

The budding yeast Saccharomyces cerevisiae expresses two Kinesin-5 homologs, Cin8 and Kip1. While they do not perform identical roles in the cell, as is evident from only Cin8 being essential for spindle formation [54], there is a large overlap in functions during mitosis $[98,99]$. One striking similarity between both Kinesin- 5 motors is the ability to move toward both the plus as well as the minus end of a microtubule [45, 46, 47, 48] (see Section 1.1). The molecular mechanism for this change of direction of motion is still unknown, but experiments show that lowering the ionic strength [46, 48] or binding between two microtubules [46, 47] switches the motor to plus-end directed motility. It was recently demonstrated by electron microscopy that in the Kinesin-5 motor from Drosophila melanogaster the head and tail domains are in close proximity [66]. We therefore propose the interaction of head and adjacent tail domains as a possible mechanism for the observed bidirectionality of $S$. cerevisiae mitotic motors.

In this chapter we investigate this hypothesis by examining the role of Cin8's tails in the regulation of its directionality and motor function. To look solely at the motility-generating subunits of Cin8, we constructed a stable dimeric Kinesin-5 head/Kinesin-1 stalk chimera, termed Cin8Kin (see also Section 1.2), consisting of head and neck linker of Cin8 fused to the stalk of D. melanogaster Kinesin-1. At the single-molecule level, Cin8Kin is bidirectional with frequent changes in direction, indicating that the Cin8 head domains are inherently bidirectional.

To remove any possible head-tail-interactions in the native tetramer, we built the Cin8 variant Cin8 $\Delta$ tail, in which we removed the tail domains. In contrast to the wild type motor, the motility of single Cin8 $\Delta$ tail molecules under high ionic-strength conditions is slow and shows no stable direction of movement. Instead, it randomly moves to either the plus or the minus end of a microtubule without any change of direction during an individual run. Furthermore, Cin8 $\Delta$ tail shows a decreased ability to crosslink two microtubules in vitro.

Based on these findings, we conclude that the interactions between tail and motor domains of Cin8 enable stable switching between plus- and minus-end directed motion and that the tails are involved in microtubule crosslinking. 


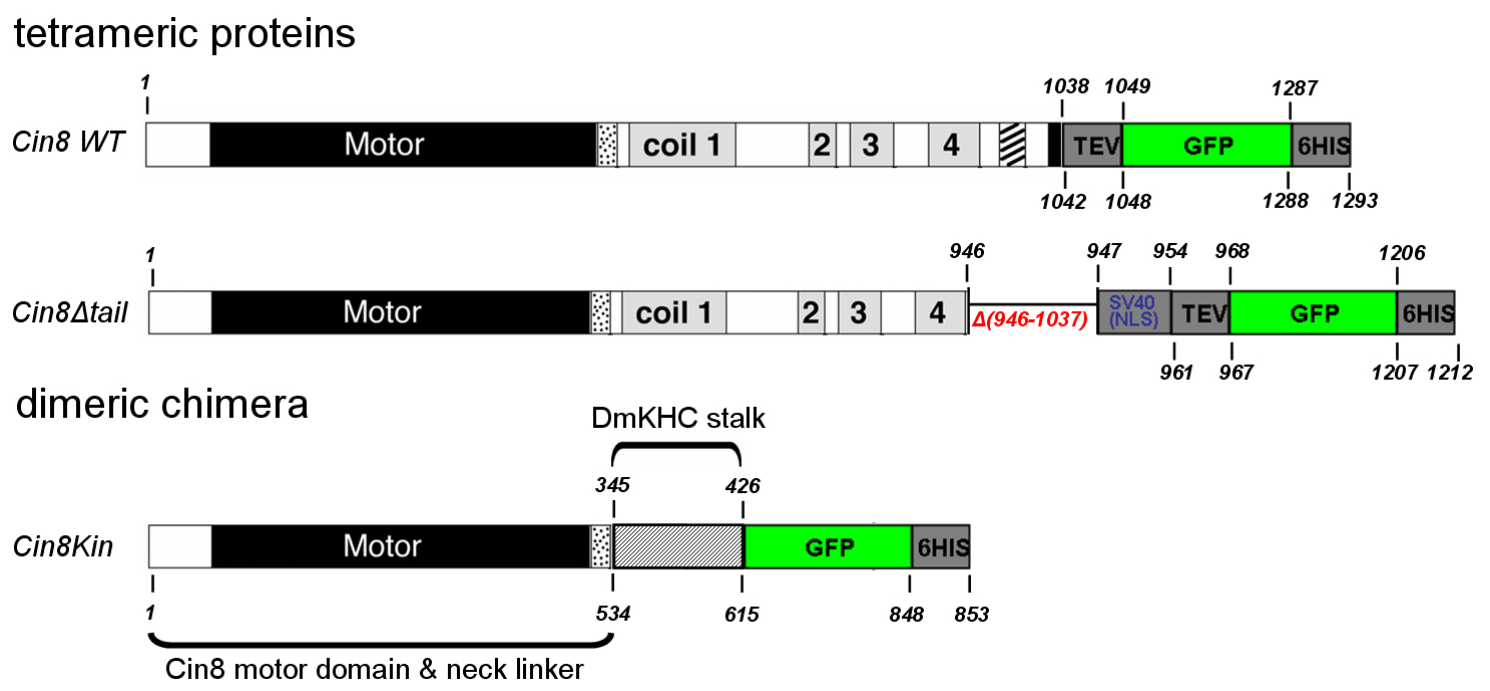

Figure 4.1: Schematic representation of Cin8 constructs

Tetrameric proteins: Structural features of the Cin8 wild type sequence with the corresponding amino acid numbers. Sequence for Cin8 $\Delta$ tail with the deletion of the tail domains indicated. Dimeric chimera: Sequence of the chimeric construct Cin8Kin. The motor domain and the neck-linker sequence of Cin8 (residues 1 - 534) were fused with the (truncated) stalk of DmKHC (DmKHC residues 345 - 426). The numbering refers to the amino acid numbering in the respective wild type motor sequences.

\section{$4.1 \quad$ Single molecules of Cin8Kin move bidirectional}

To test the hypothesis that the tail domains of Cin 8 regulate its motor functions, we examined the motility of a chimeric Cin 8 construct in which the majority of the stalk and all of the tail domains are removed. We fused the motor domain and neck linker of Cin8 (residues 1 to 534 of the Cin8 sequence) to the truncated stalk of D. melanogaster Kinesin-1 (DmKHC, residues 345 to 426) to form the chimera Cin8Kin and added a C-terminal GFP ${ }^{1}$ (Figure 4.1). In previous studies [68, 79], we demonstrated that this strategy provides us with a stable dimeric chimera in which the motor domains are not influenced by any other part of the protein (see Chapter $3)$.

To examine the effect of the stalk and tail on the directionality of Cin8, we performed single-molecule fluorescence assays (see Section 2.3) in low (80 mM PIPES) and high ionic-strength buffer $(80 \mathrm{mM}$ PIPES $+175 \mathrm{mM} \mathrm{KCl})$ with the wild type motor as well as the two constructs.

Consistent with a previous report [46], we found that the wild type Cin 8 motor $^{2}$ was minus-end directed under high ionic-strength conditions with an average velocity of $\sim 250 \mathrm{~nm} / \mathrm{s}$ (Figure 4.2 top). In a buffer with low ionic strength, Cin8 moved in an erratic manner toward the plus end of the microtubule. Between episodes of plusend directed motion, phases with minus-end motion occurred, which resulted in a

\footnotetext{
${ }^{1}$ Cin8Kin cloning, expression and purification was done by Kerstin von Roden, Charlotte Willms, and Dieter Klopfenstein.

${ }^{2}$ Single-molecule fluorescence assays with wild type Cin8 performed and analyzed by Alice Wiesbaum.
} 
wild type Cin8
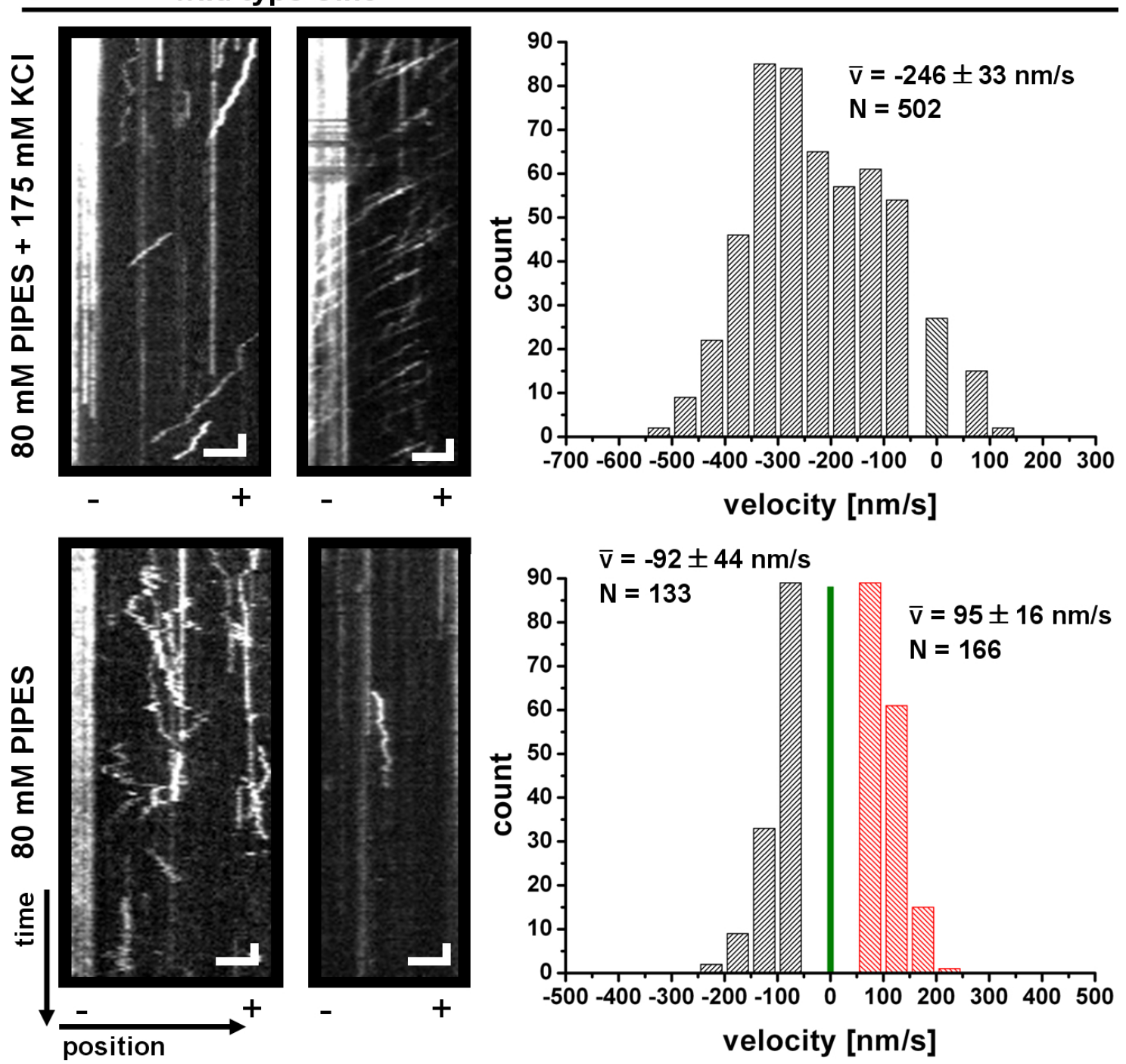

Figure 4.2: Single-molecule analysis of wild type Cin8

Single-molecule analysis of wild type Cin8 in high (upper row) and low (lower row) ionic-strength buffer. Exemplary kymographs: The atto labeled minus ends of the microtubules are on the left side. Vertical scale bars: $8 \mathrm{~s}$; horizontal scale bars: $3 \mu \mathrm{m}$.

Histogram of the velocity distribution from the segmentation analysis for wild type Cin8. High ionic strength: average minus-end velocity $-246 \pm 33 \mathrm{~nm} / \mathrm{s}$ (mean $\pm \mathrm{SD}$ ). Low ionic strength: average minus-end velocity $-92 \pm 44 \mathrm{~nm} / \mathrm{s}$, average plus-end velocity $95 \pm 16 \mathrm{~nm} / \mathrm{s}$, $\mathrm{N}$ : number of analyzed segments.

bimodal velocity distribution with two peaks at $\sim \pm 100 \mathrm{~nm} / \mathrm{s}$ (Figure 4.2 bottom). In contrast to the wild type motor, the motion of the Cin8Kin variant was slow, processive and with irregular directionality under both low and high ionic-strength conditions. Individual molecules often switched their direction of motion during processive runs (Figure 4.3). We were unable to observe any bias toward a certain direction of movement along the microtubule in both low and high ionic-strength buffer conditions (Figure 4.3). The time a motor stayed bound to a microtubule varied with ionic strength of the buffer: in high ionic strength the motor was bound for only a couple of seconds, while in low ionic strength the binding time was in excess of a minute (Figure 4.3). Apart from the change in binding times - which may be a direct consequence of the difference in electrostatic interaction between 


\section{Cin8Kin}
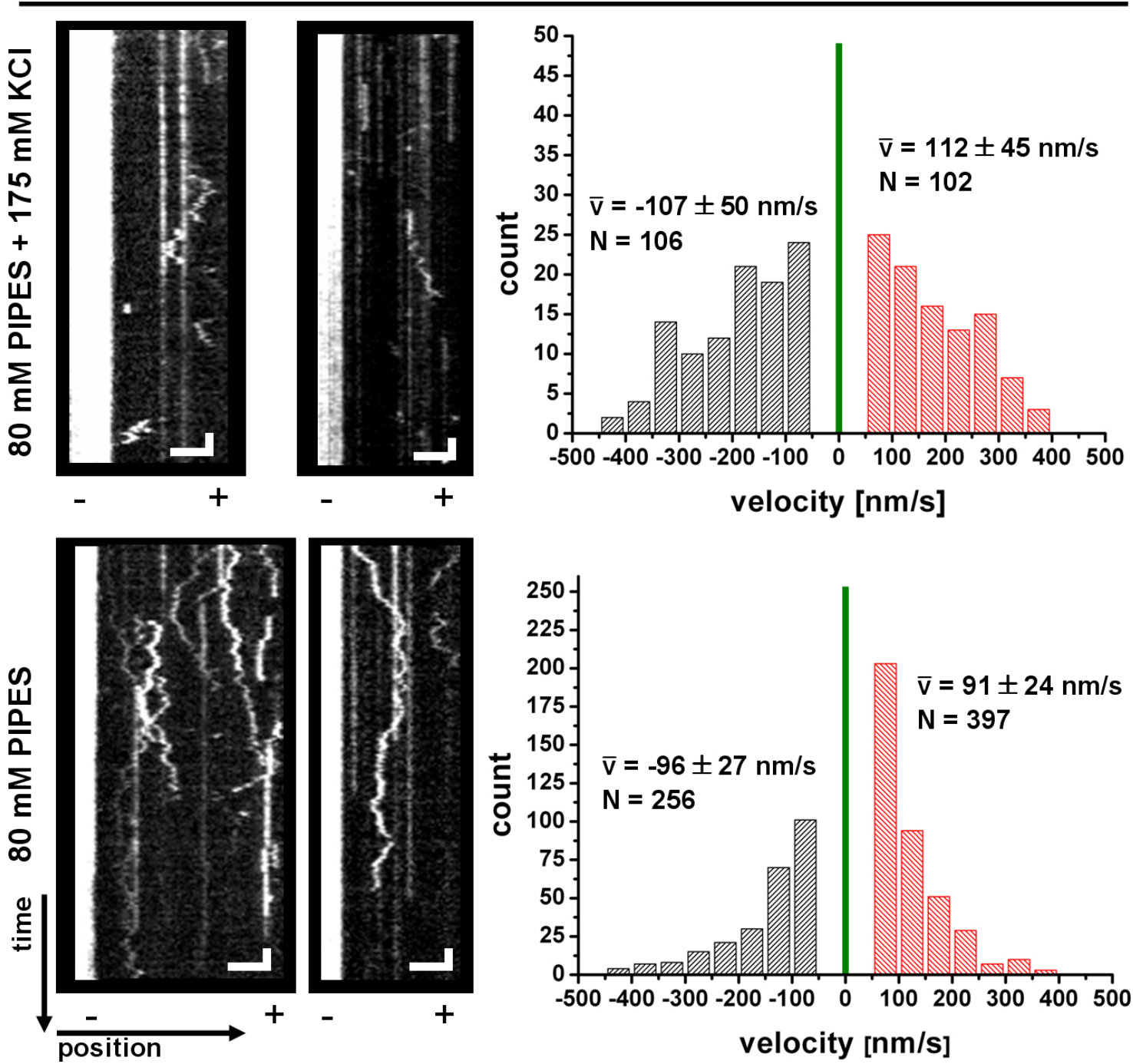

Figure 4.3: Single-molecule analysis of Cin8Kin constructs

Single-molecule analysis of Cin8Kin in high (upper row) and low (lower row) ionic-strength buffer. Exemplary kymographs: The atto labeled minus ends of the microtubules are on the left side. Vertical scale bars: $8 \mathrm{~s}$; horizontal scale bars: $3 \mu \mathrm{m}$.

Histogram of the velocity distribution from the segmentation analysis for Cin8Kin. High ionic strength: average minus-end velocity $-107 \pm 50 \mathrm{~nm} / \mathrm{s}$ (mean $\pm \mathrm{SD}$ ), average plus-end velocity $112 \pm 45 \mathrm{~nm} / \mathrm{s}$. Low ionic strength: average minus-end velocity $-96 \pm 27 \mathrm{~nm} / \mathrm{s}$, average plus-end velocity $91 \pm 24 \mathrm{~nm} / \mathrm{s}$, N: number of analyzed segments.

motor and microtubule - the bidirectional characteristics of Cin8Kin motility were insensitive to any variation of buffer ionic strength. To quantitatively analyze the velocities of Cin8Kin movement, we used a segmentation of the kymographs of the recorded motion. We divided the kymographs into segments with a window size of four frames (i.e. $3.2 \mathrm{~s}$ ) and linearly fitted the motion in these segments [46]. The velocity distributions obtained with this segmentation analysis confirm that under both high and low ionic-strength conditions, the motility of Cin8Kin is bidirectional with identical values for the plus- and minus-end directed velocities (Figure 4.3). These results indicate that the motility-generating subunits of the wild type Cin 8 molecule, i.e. the motor domains and neck linker, are able to move along a single microtubule in both plus- and minus-end directions. This suggests that not only 

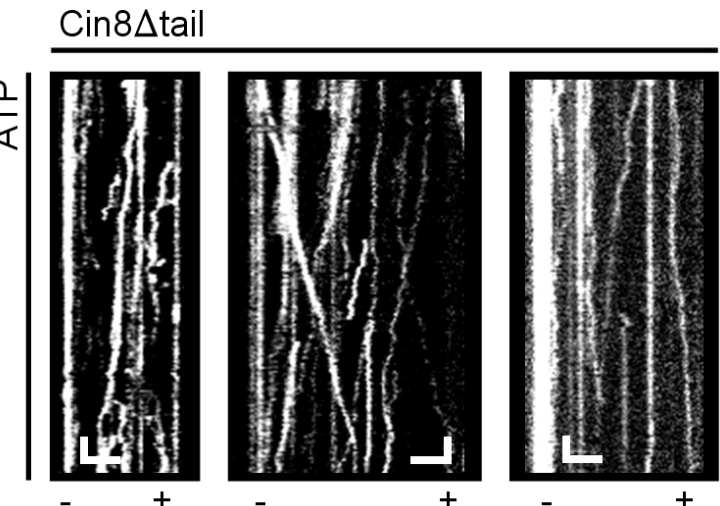

Cin8Kin
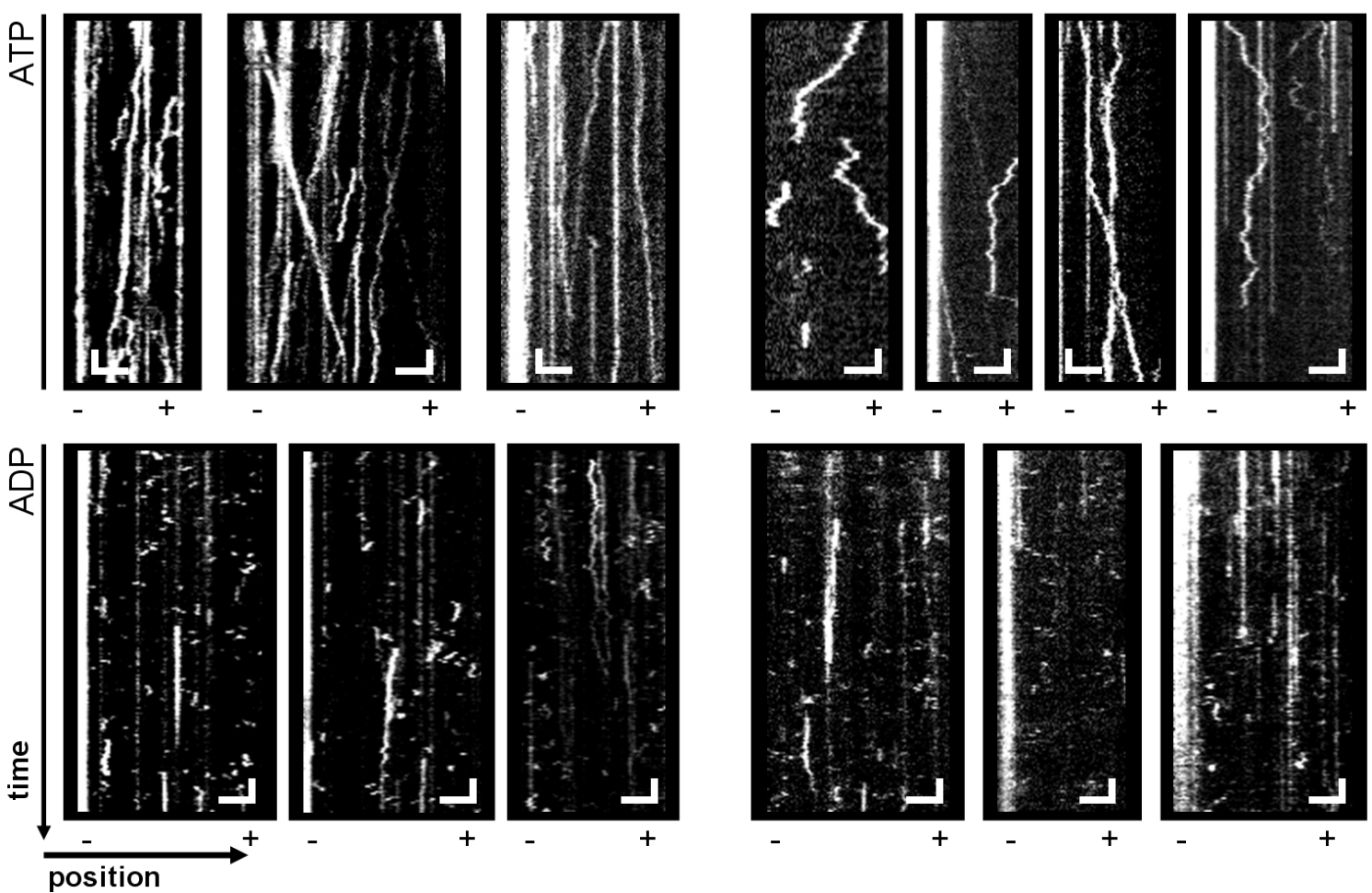

Figure 4.4: ADP-control for Cin8Kin and Cin8 $\Delta$ tail

Single-molecule fluorescence measurements of Cin8Kin and Cin8 $\Delta$ tail in the presence of ATP (top row) and ADP (bottom row). In the presence of ADP, no active, processive motion is apparent. Binding events last typically for one frame only. Cin8 $\Delta$ tail was measured in high, Cin8Kin in low ionic-strength buffer. Vertical scale bars: $8 \mathrm{~s}$; horizontal scale bars: $3 \mu \mathrm{m}$.

elements in the tail of Cin8 might be required for a stable regulation of directionality, but also elements in the stalk and/or the formation of the tetrameric complex. Control experiments with ADP instead of ATP showed that the described motion is ATP-driven (Figure 4.4) and does not result from diffusional processes.

\section{Force generation of Cin8Kin molecules}

To further analyze the motile properties of the Cin8Kin chimera, we performed single-bead assays (see Section 2.3) under low ionic-strength conditions. In the first set of experiments, we positioned a Cin8Kin coated bead on top of a microtubule. Upon binding of the motor to the microtubule, we turned the trap off and looked for any movement of the bead. In agreement with the single-fluorescence assays, the motor is able to drag beads toward both ends of a microtubule (Figure 4.5). Surprisingly, the motion of the beads in the load-free conformation was stably directed toward one of the two ends of a microtubule. We could not observe any back and forth movement. This can be explained by the relatively high concentration of motors that was used in this experiment. Multiple motors on a bead acting on one 
A

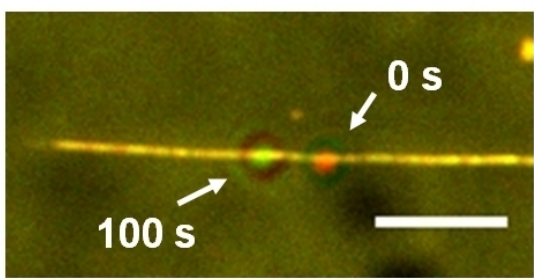

B

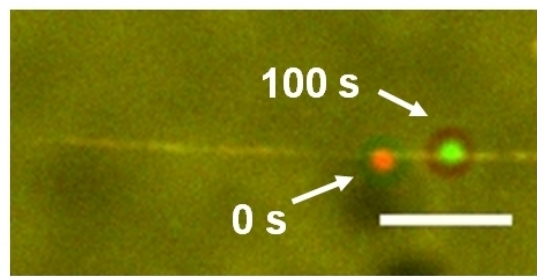

Figure 4.5: Bidirectional movement of Cin8Kin coated beads

Beads, coated with Cin8Kin molecules, are positioned on top of a fluorescently labeled microtubule. After allowing the motor to bind to the microtubule, the trap is turned off. An overlay of two frames of a video obtained when the trap was turned off is shown (first frame at $0 \mathrm{~s}$, colored red; second frame at $100 \mathrm{~s}$, colored green). A The bead moves to the left. After the first experiment, the bead was trapped again and repositioned on top of the same microtubule. B This time, the bead moves to the right, indicating the ability of the motor to walk toward both ends of a microtubule. Scale bars: $5 \mu \mathrm{m}$.

microtubule at the same time could be able to suppress the switching of direction of individual motors, giving rise to processive, unidirectional motion.
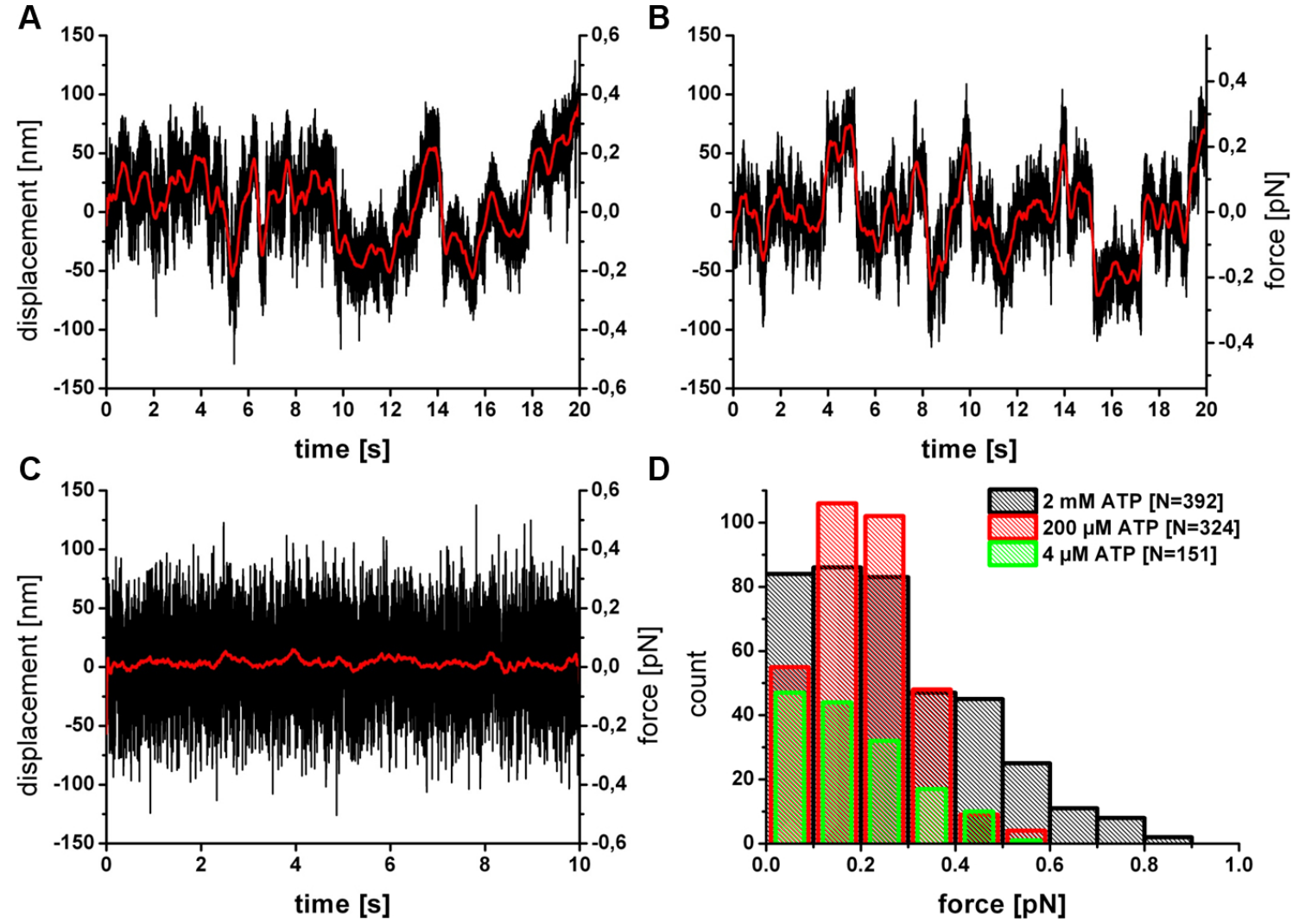

Figure 4.6: Data of single-bead assays with Cin8Kin

Traces of Cin8Kin coated beads moving in the potential of an optical trap. Black: raw data; red: data median filtered with a 0.03 s sliding window. A Exemplary results of a single-bead assay with $\mathrm{Cin} 8 \mathrm{Kin}$ in the presence of $2 \mathrm{mM}$ ATP. B Exemplary results of the assay in the presence of $4 \mu \mathrm{M}$ ATP. Note that both traces lack the typical instantaneous unbinding events. C Shown for comparison: calibration measurement of a bead in solution. D Histogram of forces Cin8Kin reaches before reversing its movement direction. Forces are typically lower than $0.5 \mathrm{pN}$. N: number of analyzed turning points. 


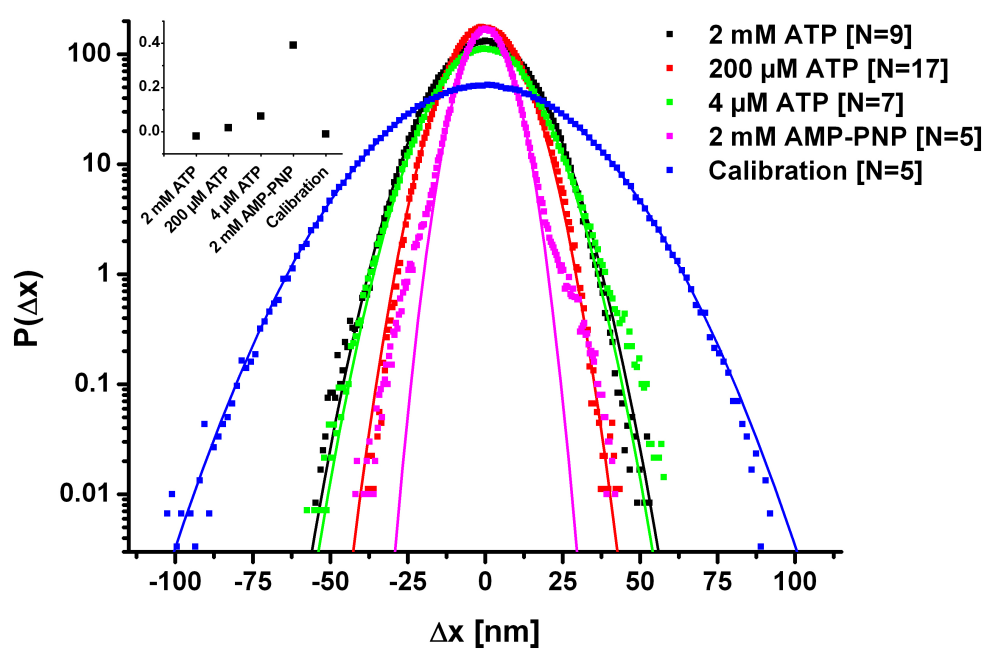

Figure 4.7: Van Hove correlation functions for Cin8Kin

Ensemble-averaged van Hove correlation functions of single-bead assays with Cin8Kin under different nucleotide condition (symbols). Most distributions approximately agree with a Gaussian (solid line). Two observations are evident: (i) The distributions for the active motor measurements, i.e. in the presence of ATP, lie between the calibration and the immobile motor (AMP-PNP). (ii) Only the measurement in the presence of AMP-PNP clearly deviates from a Gaussian distribution. This is supported by the inset, showing the non-Gaussian parameter. N: number of analyzed measurements (120 s each).

While the motor showed distinct bidirectional movement patterns when not under load, this behavior changed when loaded. With the restraining force of the optical trap present, only short, erratic excursions of the bead from the center of the trap could be observed (Figure $4.6 \mathrm{~A}$ ). The shape of the motion was largely unaffected by a decrease of ATP concentration (Figure 4.6 B). Neither a clear direction of motion nor any rapid unbinding events could be identified for different ATP concentrations. In these experiments, Cin8Kin reached maximum forces of typically less than $0.5 \mathrm{pN}$ before changing its direction of motion (Figure $4.6 \mathrm{D}$ ). The motor concentration was lowered until less than half of the beads in the experiments showed activity. This ensures that only single motors interact with the microtubule at a given time (see also Section 2.3). To be able to record any movement at all, a trap stiffness in the order of $10^{-3} \mathrm{pN} / \mathrm{nm}$ had to be used. At higher trap stiffnesses, no motion was apparent (see also Figure 4.8).

To investigate the detailed statistical properties of the movement of the Cin8Kin motors, we calculated the ensemble averaged van Hove correlation function for the motion of the beads. The van Hove correlation $\mathrm{P}(\Delta \mathrm{x}(\tau))$ is defined as the probability distribution of the beads displacements at a given lag time $\tau$ [100, 101]:

$$
P(\Delta x(\tau)), \Delta x(\tau)=x(t+\tau)-x(t) .
$$

It is obtained by dividing the frequency histogram of $\Delta \mathrm{x}(\tau)$ by the product of the total number of samples and the binning size of $\Delta \mathrm{x}$ in units of nm. For purely thermal fluctuations, the distribution of the observed bead trajectories should follow a Gaussian shape. The lowest order deviation of the van Hove correlation func- 
A

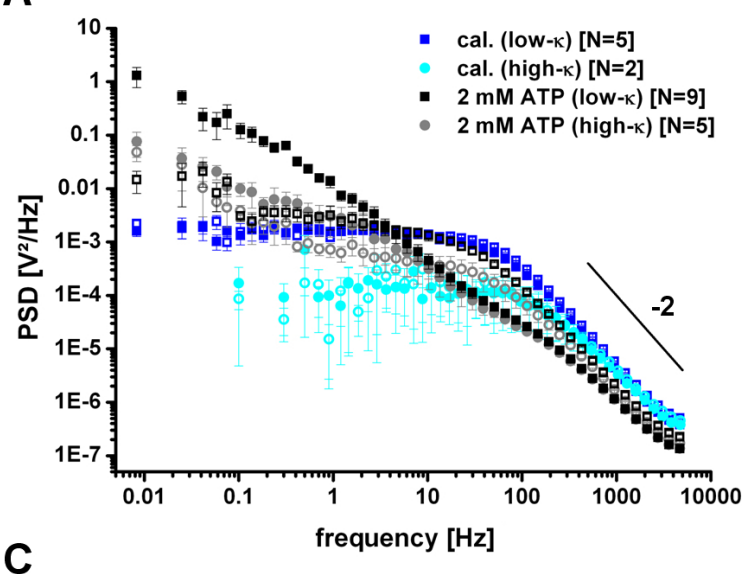

C

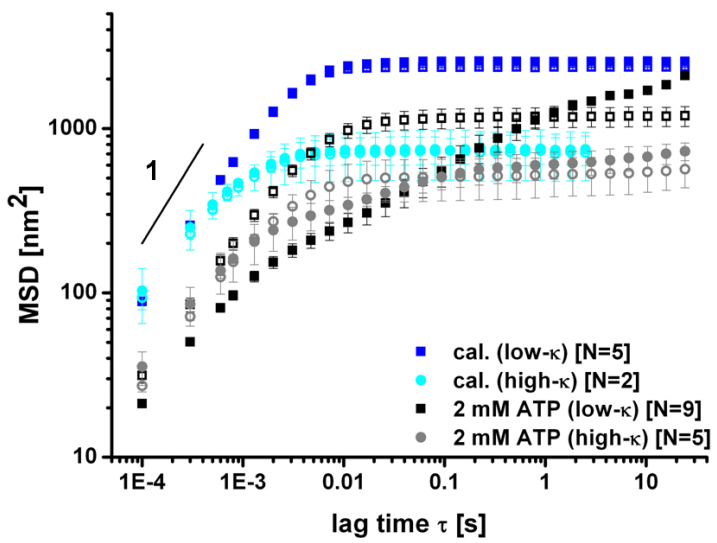

B
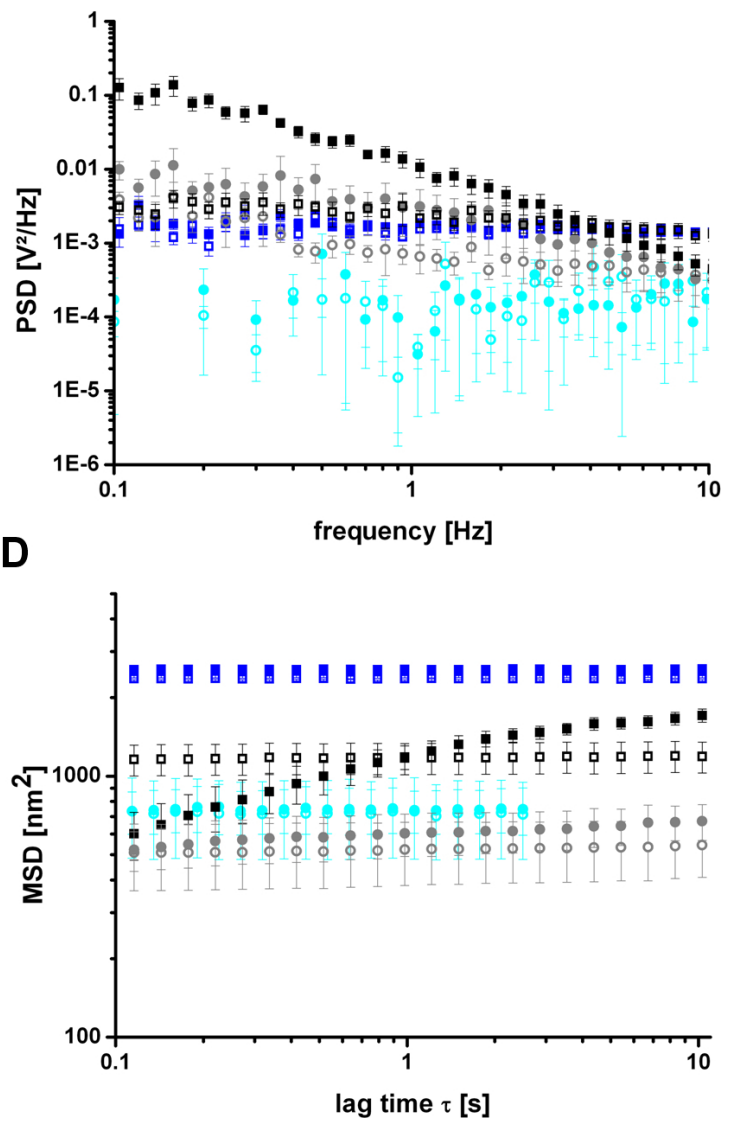

Figure 4.8: PSD and MSD analysis of Cin8Kin at different trap stiffnesses

Open symbols correspond to motion of the bead perpendicular to the microtubule. A Power spectral density calculated for Cin8Kin at different trap stiffnesses (mean \pm SEM). Note that only the signal for $2 \mathrm{mM}$ ATP parallel to the microtubule (filled black symbols) shows distinct activity in the low-frequency regime. B PSD plotted from 0.1 to $10 \mathrm{~Hz}$, the typical frequency range for motor activity. C Mean square displacement calculated for Cin8Kin at different trap stiffnesses (mean \pm SEM). Again, only the filled black symbols (2 mM ATP) deviate from the trap-confined diffusion visible in the other data sets. D MSD plotted from 0.1 to $10 \mathrm{~s} \mathrm{lag}$, the typical time scale for motor activity. N: number of analyzed measurements (120 s each).

tion from a Gaussian is quantified by the one-dimensional non-Gaussian parameter (NGP) [101, 102]:

$$
N G P=\frac{\left\langle\Delta x(\tau)^{4}\right\rangle}{3\left\langle\Delta x(\tau)^{2}\right\rangle^{2}}-1
$$

where $\langle\ldots\rangle$ indicates the ensemble average. The NGP can be positive or negative and vanishes for a Gaussian distribution. Thus it represents the degree of nonGaussianity of a distribution.

In Figure 4.7, the van Hove correlation functions for measurements of Cin8Kin under different nucleotide conditions are shown. For the lag time $\tau$ a value of $1 \mathrm{~s}$, the typical timescale for motor motion, was chosen. The NGP for the analyzed experiments is shown in the inset of Figure 4.7. For the calibration measurement, the measured fluctuations of the beads are purely thermal, hence the distribution of the observed bead trajectories was almost Gaussian (NGP $\approx 0.01$ ). The position distributions of the beads for the actual experiments containing ATP, while having 
A

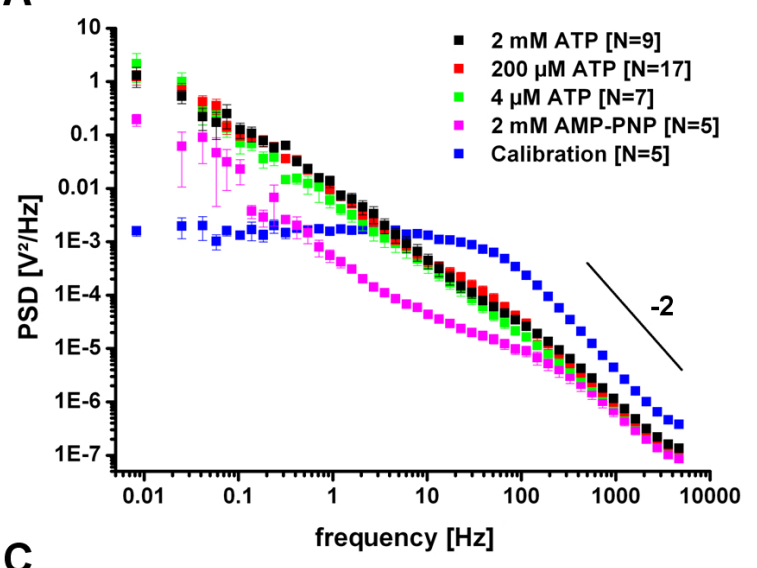

C

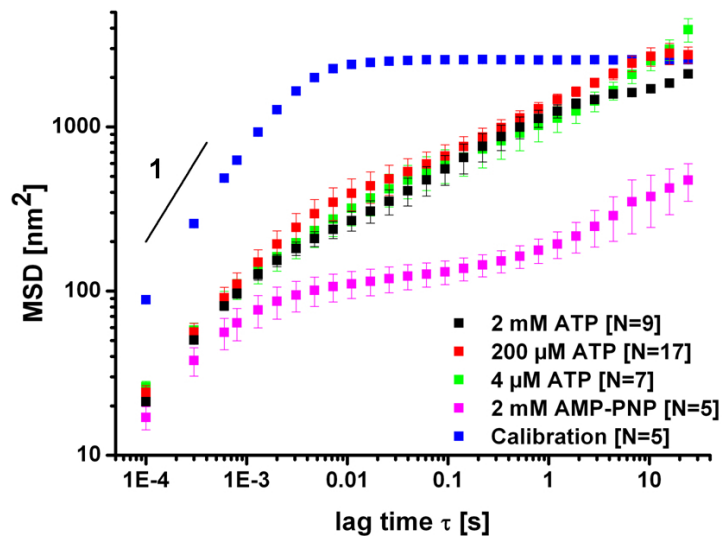

B
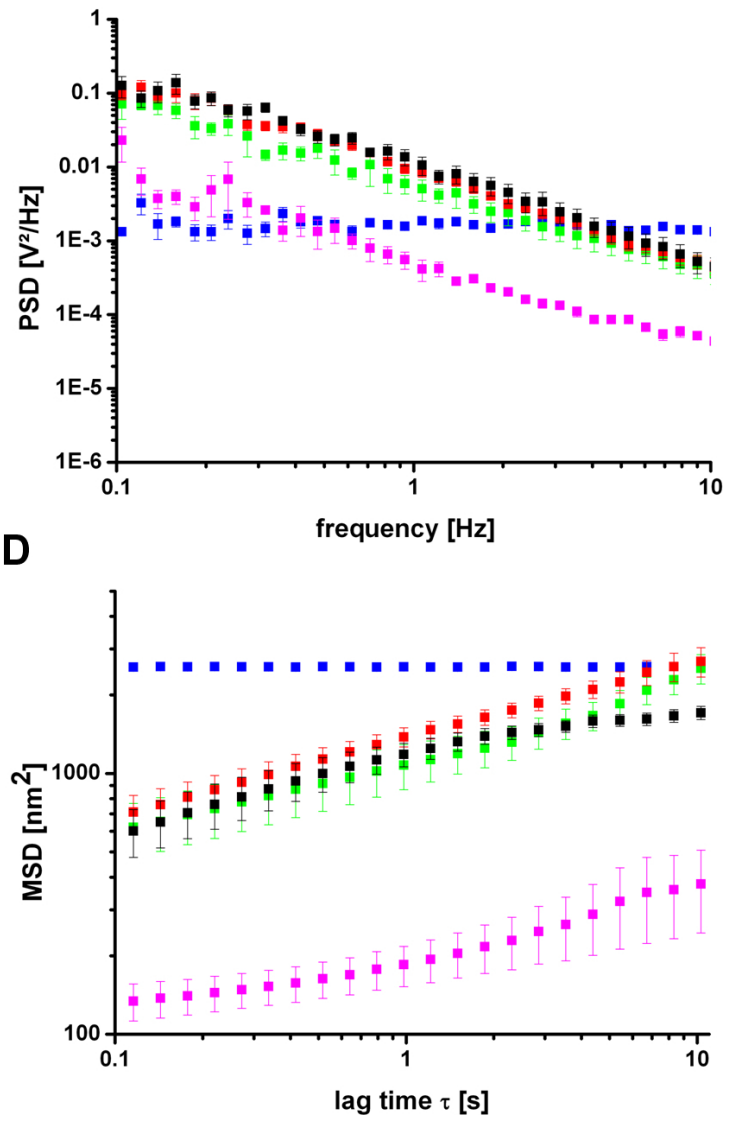

Figure 4.9: PSD and MSD analysis of Cin8Kin for different nucleotide conditions A Power spectral density calculated for Cin8Kin under different nucleotide conditions (mean \pm SEM). Note that while the measurements for the different ATP concentrations are similar to each other, they largely differ from the experiments in the presence of AMP-PNP. B PSD plotted from 0.1 to $10 \mathrm{~Hz}$, the typical frequency range for motor activity. The slope for the measurements containing ATP is $-1.25 \pm 0.02$ on average. C Mean square displacement calculated for Cin8Kin under different nucleotide conditions (mean $\pm \mathrm{SEM}$ ). Again, the measurements for the different ATP concentrations are similar, while differing from the none-motile conditions. D MSD plotted from 0.1 to $10 \mathrm{~s} \mathrm{lag}$, the typical time scale for motor activity. The slopes for the measurements containing ATP signals are $0.29 \pm 0.01$ on average. N: number of analyzed measurements $(120 \mathrm{~s}$ each).

been smaller than for the calibration measurement, still followed a Gaussian shape $(2 \mathrm{mM}$ ATP: NGP $\approx-0.02,200 \mu \mathrm{M}$ ATP: NGP $\approx 0.02,4 \mu \mathrm{M}$ ATP: NGP $\approx 0.0$ ). Only in the presence of AMP-PNP did the van Hove correlation function deviate from a Gaussian distribution (NGP $\approx 0.39$ ). While a small tendency to larger values of the NGP for lower ATP concentrations might be evident, the deviations from thermal behavior observed for Cin8Kin in the presence of varying amounts of ATP were to small to reliably detect any differences. However, the narrower distributions in comparison with the calibration measurement as well as the results from the experiment with AMP-PNP clearly argue for an active movement of Cin8Kin in the single-bead motility assays.

To study the details of the motile properties of Cin8Kin in the trap, we performed extensive frequency analysis of measurements at different trap stiffnesses (Figure 4.8 ) as well as under different nucleotide conditions (Figure 4.9). The position of 
beads in single-bead assays were measured for $120 \mathrm{~s}$. From these signals, the power spectral density (PSD) and the mean square displacement (MSD) of the bead for each experiment was calculated.

Trap stiffnesses of $\sim 0.003 \mathrm{pN} / \mathrm{nm}$ and $\sim 0.03 \mathrm{pN} / \mathrm{nm}$ have been used. The motility of Cin8Kin motors was only evident in the low-frequency part of the data at the low trap stiffness (filled black symbols in Figure 4.8). The signal at the high trap stiffness is similar to the calibration measurement (most notably in Figure 4.8 C), as are the data sets from the motion perpendicular to the microtubule (open symbols in Figure 4.8).

For different nucleotide conditions, ATP concentrations in a range from $2 \mathrm{mM}$ to $4 \mu \mathrm{M}$ as well as $2 \mathrm{mM}$ of AMP-PNP have been used. Since the Michaelis-Menten constant $\mathrm{K}_{M}$ of the ATP turnover reaction for Cin8 is $15 \mu \mathrm{M}$ ATP [46], the ATP concentrations used provided us with conditions well below and above the $\mathrm{K}_{M}$ value. Interestingly, the analysis showed that the reduction of ATP in the motility buffer does not influence the movement of the motor. The calculated PSDs were largely similar. However, when we exchanged ATP for AMP-PNP in the motility buffer, the movement behavior of Cin8Kin was altered (Figure 4.9 A and B). A similar behavior was observed for the MSD analysis. The MSDs for the various ATP concentrations were largely identical. The data scales with an exponent of less than one $(\sim 0.3)$, suggesting sub-diffusive motion (Figure $4.9 \mathrm{C}$ and $\mathrm{D}$ ). The observation, that the beads motion was altered when exchanging ATP for AMP-PNP, suggest that we actually observed active motion. This motion appears to be sensitive to applied loads as is evident from the disappearance of the motion at higher trap stiffnesses. Why this motion seems to be unaffected by a variation of the ATP concentration from $2 \mathrm{mM}$ to $4 \mu \mathrm{M}$ is unclear.

Taking all the gathered results into account, we hypothesize that the motor is actively moving bidirectionally in a back and forth manner inside the trap. The Cin8 motor domains without the adjacent tails are unable to preserve a certain direction of movement over a longer period of time. In addition, the introduction of a force apparently increases the probability of a change of direction. Cin8Kin reverses movement direction upon reaching forces of $\sim 0.5 \mathrm{pN}$. An excursion from the trap that equals $0.5 \mathrm{pN}$ at typically used trap stiffnesses corresponds to less than $100 \mathrm{~nm}$ of movement. Since this is below the resolution of the TIRF microscope $(1 \mathrm{px} \approx 160 \mathrm{~nm})$, the motion we observed in the single-molecule fluorescence assay was suppressed in the trapping experiment. This also explains why we could not see any motor activity at higher trap stiffnesses: The motor changed its direction of motion before the movement of the bead inside the trap was distinguishable from the noise.

It seems that Cin8Kin changes its direction of motion sooner when in a loaded conformation. This might point to a force sensitive regulation mechanism for the movement direction of wild type Cin8. 


\section{Cin8 $\Delta$ tail}
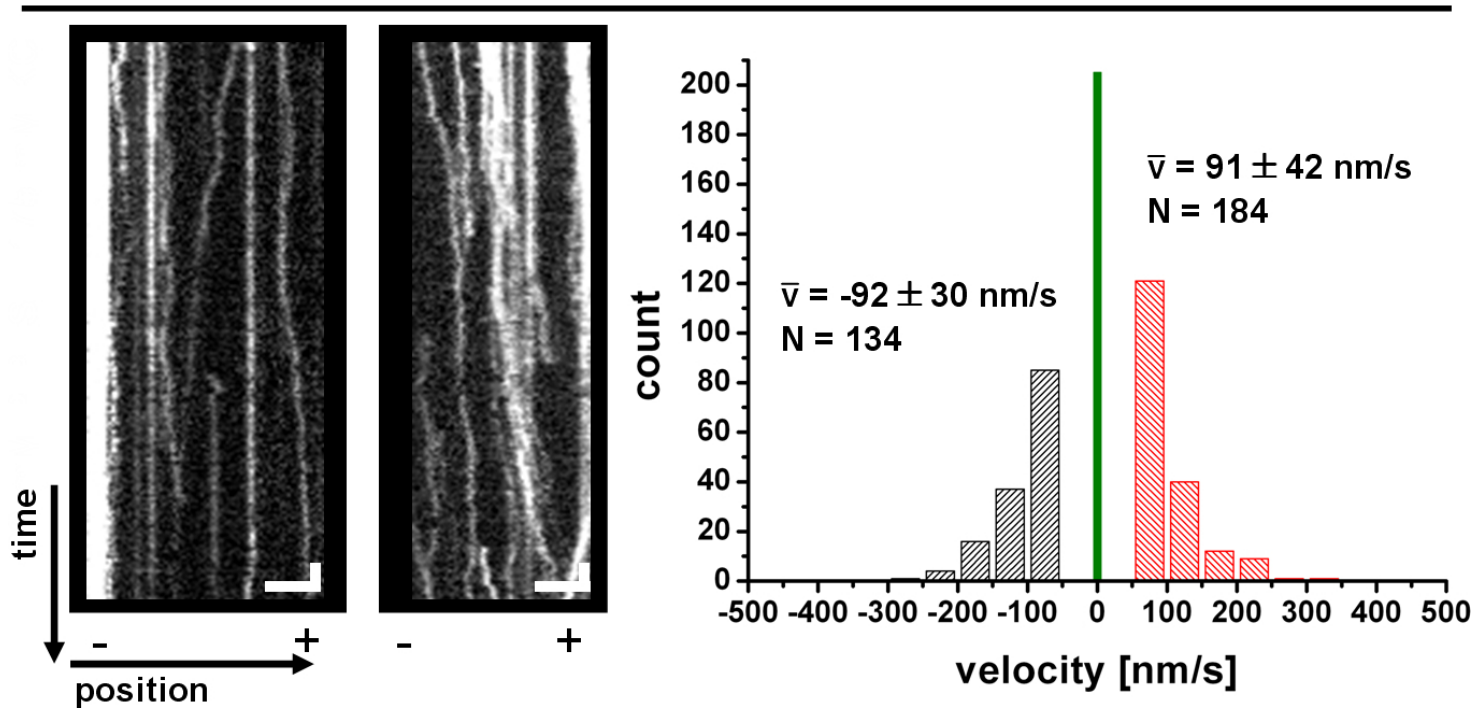

Figure 4.10: Single-molecule analysis of Cin8 $\Delta$ tail construct

Single-molecule analysis of Cin8 $\Delta$ tail in high ionic-strength buffer (80 mM PIPES $+175 \mathrm{mM}$ $\mathrm{KCl}$ ). Exemplary kymographs: The atto labeled minus ends are on the left side. Vertical scale bars: $8 \mathrm{~s}$; horizontal scale bars: $3 \mu \mathrm{m}$.

Histogram of the velocity distribution from the segmentation analysis for Cin8 $\Delta$ tail. Average minus-end velocity $-92 \pm 30 \mathrm{~nm} / \mathrm{s}$ (mean $\pm S D$ ), average plus-end velocity $91 \pm 42 \mathrm{~nm} / \mathrm{s}, \mathrm{N}$ : number of analyzed segments.

\section{Cin8 tail domains regulate directionality}

To investigate if the tail domains are involved in the regulation of Cin8 directionality, we examined the motility of a "tailless" Cin8 variant in which the tail domains were deleted. For this purpose, we removed residues 946 to 1037 from the sequence of the wild type Cin 8 and fused it to a C-terminal GFP to build the Cin $8 \Delta$ tail motor $^{3}$ (Figure 4.1 on page 46 ). We examined Cin8 $\Delta$ tail's properties in single-molecule fluorescence assays ${ }^{4}$ (see Section 2.3).

In contrast to wild type Cin8, Cin8 $\Delta$ tail showed no motility in buffer solutions with a salt concentration below that of $80 \mathrm{mM}$ PIPES $+175 \mathrm{mM} \mathrm{KCl}$. In high ionic-strength buffer (80 mM PIPES + $175 \mathrm{mM} \mathrm{KCl})$, Cin8 $\Delta$ tail processively moved along single microtubules. The time individual Cin8 $\Delta$ tail proteins stayed bound to a microtubule were typically longer than the recording time ( $>160$ seconds, Figure 4.10). This indicates that Cin8 $\Delta$ tail is a highly processive motor.

To make sure that the measured motility was actively driven by ATP hydrolysis, we performed ADP-control experiments. In the presence of ADP, no single molecule runs were detectable (Figure 4.4 on page 49). To rule out the possibility of aggregate formation, we performed photo bleaching analysis ${ }^{5}$. In bleaching step analysis

\footnotetext{
${ }^{3}$ Cin8 $\Delta$ tail and Cin8 WT cloning and expression was done by Vladimir Fridman, purification was performed by Kerstin von Roden and Charlotte Willms

${ }^{4}$ Single-molecule fluorescence assays with Cin8 $\Delta$ tail performed and analyzed by Christina Thiede. ${ }^{5}$ Photo-bleaching experiments performed by Christina Thiede
} 
A

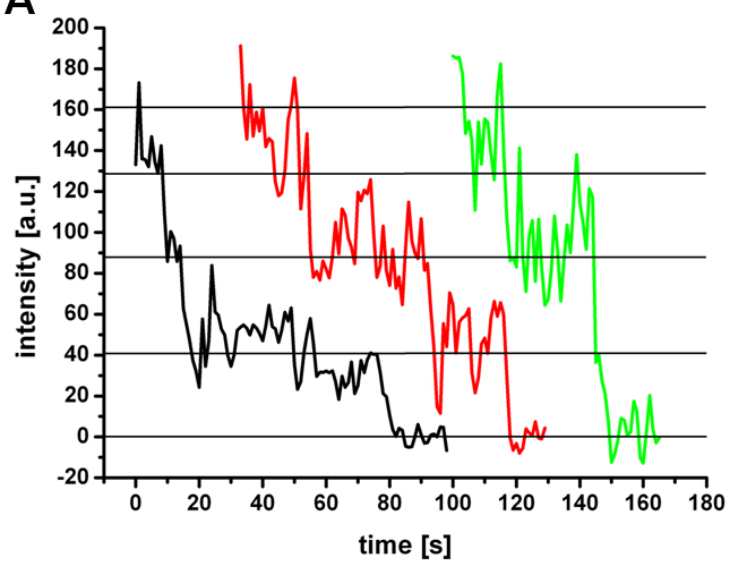

B

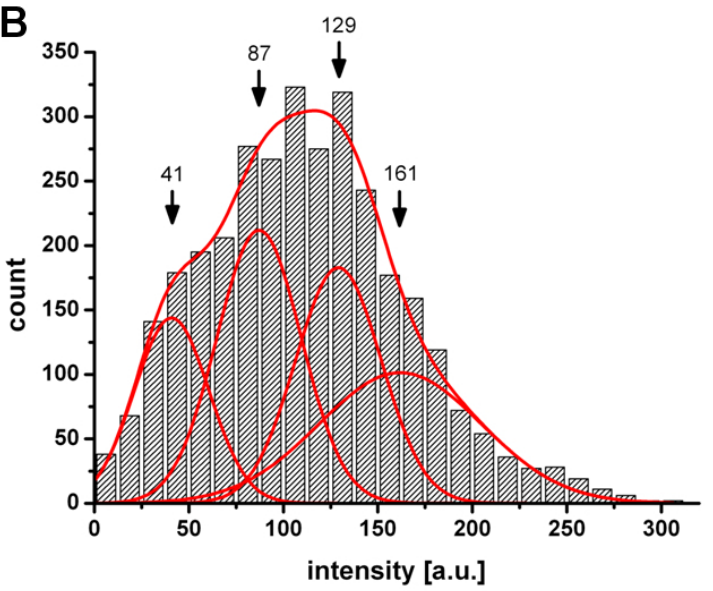

Figure 4.11: Photo-bleaching analysis for Cin8 $\Delta$ tail

A Exemplary bleaching curves for three Cin $8 \Delta$ tail molecules. B Fluorescence Intensity distribution for all measured Cin8 $\Delta$ tail molecules. The positions of the intensity peaks corresponding to one to four GFP fluorophores on a protein are indicated. The maximum of the distribution is between two and three which rules out larger aggregates of the tetrameric motor.

of single Cin8 $\Delta$ tail molecules, more than four bleaching steps were never observed (Figure $4.11 \mathrm{~A}$ ), consistent with the presence of four GFP molecules in a Cin8 $\Delta$ tail complex. In addition, the distribution of fluorescence intensities of Cin8 $\Delta$ tail-GFP molecules could be fitted with four Gaussians. The fluorescence intensity distribution shows that the measured proteins are, on average, decorated with three GFP fluorophores (Figure $4.11 \mathrm{~B}$ ). These results are consistent with Cin8 $\Delta$ tail forming a tetramer and argue against the presence of aggregates in our samples.

We found that under the high ionic-strength conditions, Cin8 $\Delta$ tail actively moved along microtubules in a bidirectional fashion. In contrast to Cin8Kin, individual Cin8 $\Delta$ tail molecules moved processively in either the plus- or minus-end direction on the microtubule, without changing the direction of movement during individual processive runs (Figure 4.10). In fact, no events were recorded where a Cin8 $\Delta$ tail protein changed its direction of movement. This behavior is different from the bidirectional movements of the dimeric Cin8Kin variant (Figure 4.3 on page 48) and the wild type Cin $8^{6}$ under low ionic-strength conditions (Figure 4.2 on page 47). To quantify the differences in the movement patterns of the various Cin 8 constructs, we calculated the autocorrelation for the recorded movement of the constructs (Figure 4.12). We found that the correlation coefficient for wild type Cin8 and Cin8 $\Delta$ tail under high ionic-strength conditions slowly decays to zero, compared to the correlation coefficient of the other variants which abruptly decreases to zero. This shows that wild type Cin 8 in high ionic strength and Cin8 $\Delta$ tail move in a persistent direction while the movement of Cin8Kin and wild type Cin8 in low ionic strength is characterized by frequent changes in the direction of movement.

Segmented analysis of Cin8 $\Delta$ tail kymographs revealed that the average velocity for plus- or minus-end motility were similar with $91 \pm 42 \mathrm{~nm} / \mathrm{s}$ and $-92 \pm 30 \mathrm{~nm} / \mathrm{s}$ (mean \pm SD), respectively (Figure 4.10). The overall motion exhibited a slight

${ }^{6}$ Single-molecule fluorescence assays with wild type Cin8 performed and analyzed by Alice Wiesbaum. 


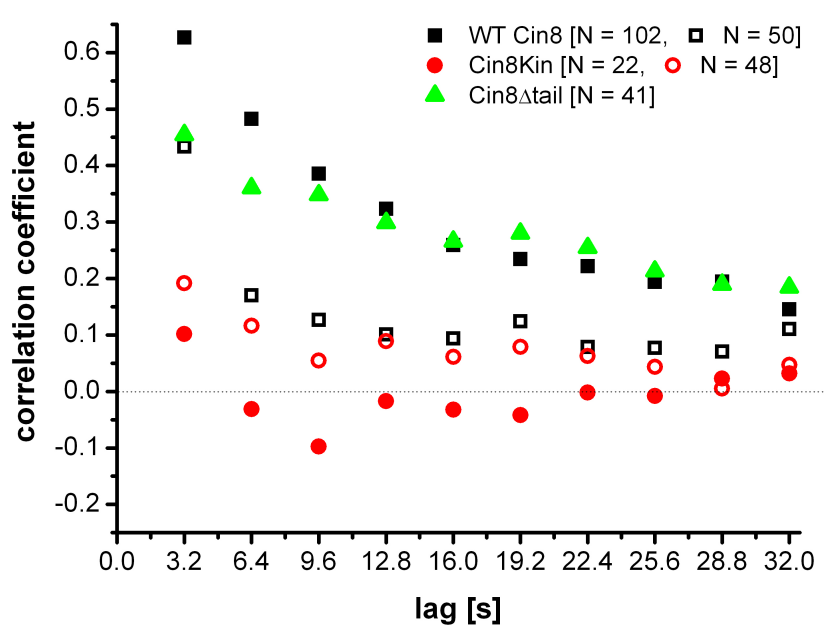

Figure 4.12: Correlation analysis of the different Cin8 constructs

Autocorrelation of single-molecule fluorescence assays with Cin8 $\Delta$ tail, wild type Cin8, and Cin8Kin in high and low ionic-strength buffer (filled symbols: high ionic-strength buffer, open symbols: low ionic-strength buffer). The curves for Cin8Kin and wild type Cin8 in low ionic-strength buffer are always close to zero, while Cin8 8 tail and wild type Cin8 in high ionic-strength buffer are significantly higher, indicating a less randomly oriented direction. Values for zero seconds lag omitted. $\mathrm{N}$ : number of analyzed motor traces.

bias toward the plus end of the microtubules. In the presence of $2 \mathrm{mM}$ ADP, only transient binding events were observed, typically shorter than three seconds (Figure 4.4 on page 49 ), indicating that the processive movements of Cin8 $\Delta$ tail in both plusand minus-end direction are ATP-dependent.

The reason behind the lack of activity from the motor in low ionic-strength buffer is not clear. In the Kinesin-5 Eg5 from Xenopus laevis, the tail domains carry an additional microtubule-binding site $[62,67]$. This could also be true for Cin8 and could explain a reduced microtubule affinity. The reasonable affinity for microtubules of Cin8Kin argues against this explanation, though. Perhaps the apparent loss of function of Cin8 $\Delta$ tail in low ionic-strength buffer points toward a different regulatory mechanism that inactivates the motor under certain conditions. The Kinesin- 5 Eg5 was reported to be highly (down)regulated when not bound to two different microtubules [62] (see Chapter 5). A similar mechanisms could be involved in Cin8 regulation. Assuming this regulation requires the tetrameric structure of the protein, this notion is supported by the lack of a similar response to buffer conditions in Cin8Kin.

The data on Cin8 $\Delta$ tail motility presented here strongly suggests that the tail domains of Cin8 are required for a stable regulation of its directionality. Under high ionic-strength conditions, the Cin $8 \Delta$ tail construct looses the minus-end directed bias of the wild type motor and becomes bidirectional. Thus, we conclude that the removal of the tail domains in Cin8 disrupts the stable regulation of the direction of motion that is apparent in the wild type protein.

\section{Cin8 tail domains are required for microtubule crosslinking}

The majority of the mitotic functions of Kinesin-5 motors are attributed to their ability to crosslink and slide apart antiparallel spindle microtubules [8, 9, 46]. In addition, binding between two antiparallel microtubules was shown to be one of the major factors that regulate the switching of the direction of movement of Cin8: 

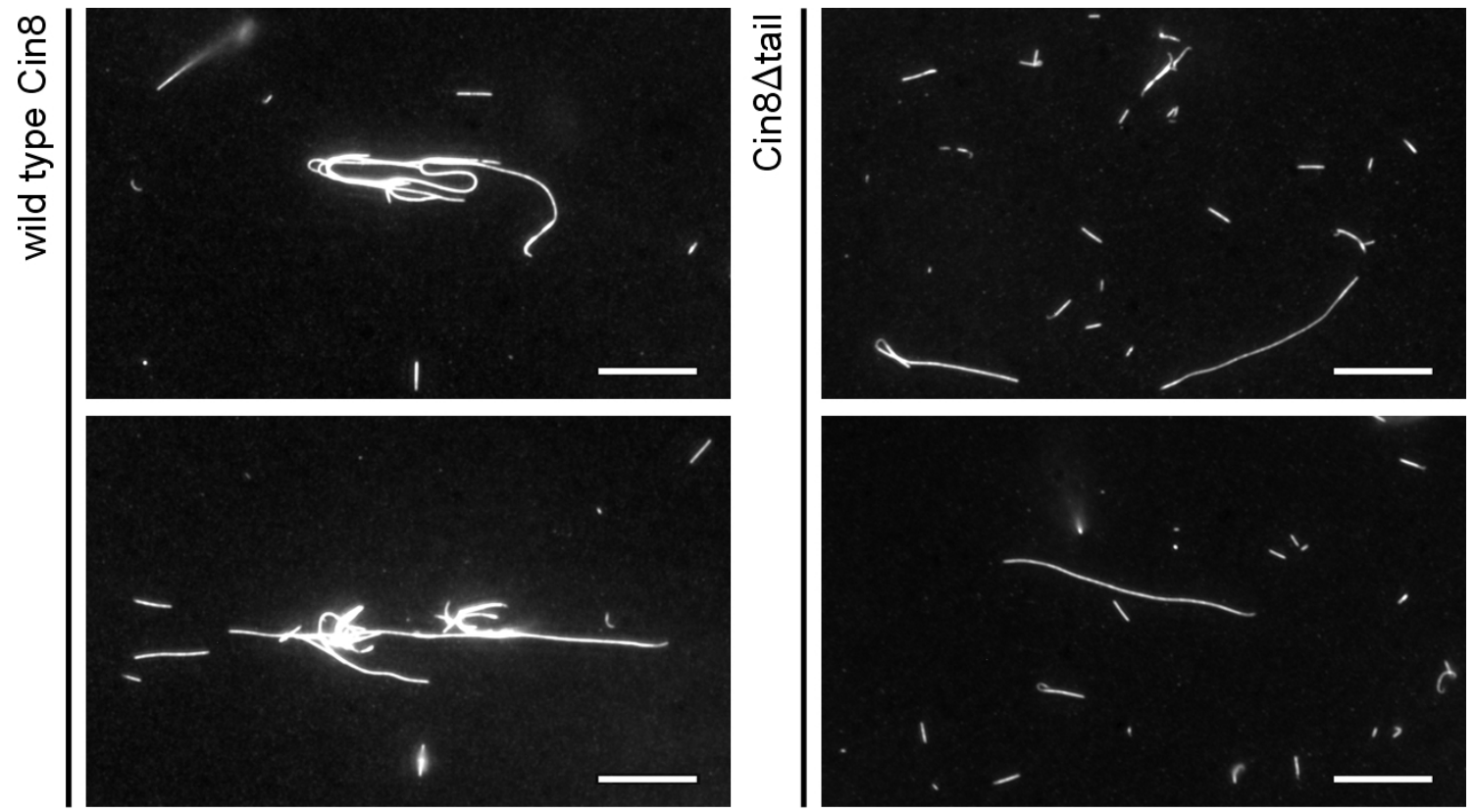

Figure 4.13: Microtubule crosslinking experiment

Microtubule crosslinking experiment with wild type Cin8 (left side) and Cin8 $\Delta$ tail (right side). In the presence of AMP-PNP, the motors were mixed with microtubules in high ionic-strength buffer. Incubation for $5 \mathrm{~min}$ allowed the motors to bind to and crosslink microtubules. Afterwards the solution was filled into assay chambers build with DETA coated cover slips. The microtubule bundles, sticking to the DETA surface, were imaged with an epi-fluorescence microscope. Scale bars: $10 \mu \mathrm{m}$.

While Cin8 moves toward the minus end of a single microtubule in high ionicstrength buffer, it switches to plus-end directed motion upon binding of a second microtubule $[46,69]$. Thus, we examined the ability of Cin8 $\Delta$ tail to crosslink and slide apart antiparallel microtubules. Firstly, we performed an experiment in which we mixed wild type Cin8 or Cin8 $\Delta$ tail with microtubules in the presence of AMPPNP, inducing stable binding of the motors to the microtubules. To our surprise, while we observed extensive bundling of microtubules by the wild type Cin8, no bundling of microtubules was observed in the presence of the Cin $8 \Delta$ tail variant (Figure 4.13). This indicates that the tailless Cin8 construct is impaired in its ability to bind two microtubules. To further investigate the bundling by Cin8 8 tail we performed relative-sliding assays ${ }^{7}$ with Cin8 $\Delta$ tail (see Section 2.3 ). Since bundling of microtubules was not achieved by Cin8 $\Delta$ tail alone, we loosely crosslinked the microtubules by adding $\sim 4 \mathrm{nM}$ of Ase1 to the solution. Ase1 is a protein present in yeast cells that is able to diffuse along the microtubule lattice while stably crosslinking two microtubules $[63,103]$. Only with the addition of Ase1 could we detect interactions of Cin8 $\Delta$ tail with two microtubules at the same time.

In Figure 4.14 A, an overlay of two frames from a relative-sliding assay is shown. As is evident from the kymographs in Figure $4.14 \mathrm{~B}$, the central microtubule that crosses two other microtubules was moved by Cin8 8 tail motors. The athermal nature of this motion is apparent, when we calculate the energy that is required

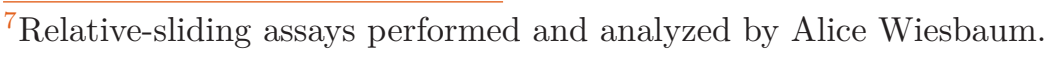



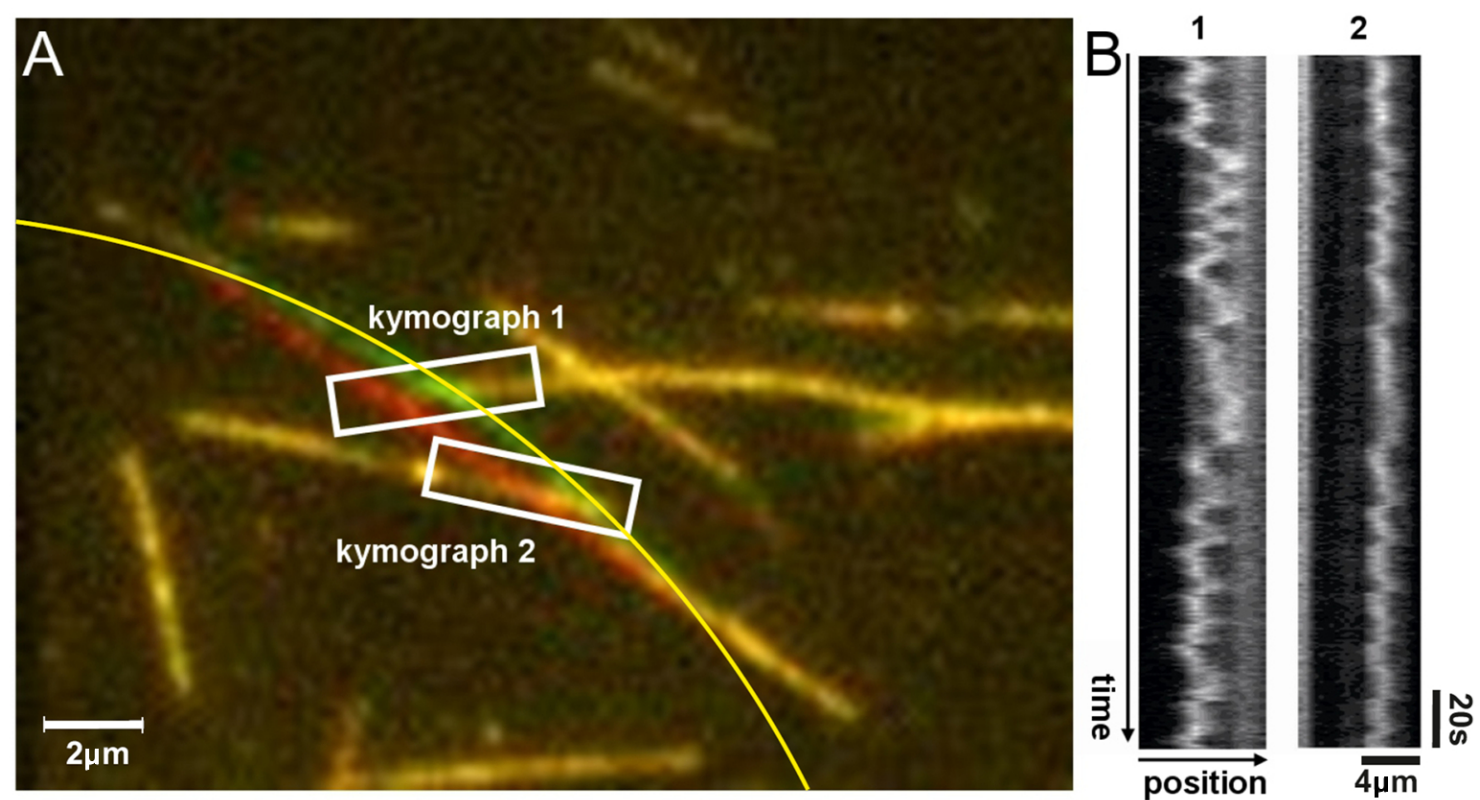

Figure 4.14: Relative-sliding assay with Cin8 $\Delta$ tail

A Overlay of two frames of a video captured during a relative sliding experiment. $4.2 \mathrm{nM}$ Ase1 have been added to the solution. The long microtubule is fixed at both ends. It crosses two surface-immobilized, shorter microtubules. At the overlap zone, actively driven motion is observed (visible by the change in microtubule position between two frames (red/green)). From the contour of the bent microtubule, approximated by the indicated circle segment, we calculated a bending energy of $\sim 35 \mathrm{k}_{\mathrm{B}} \mathrm{T}$, indicating active motion. B Kymographs of the overlap zones indicated in $\mathrm{A}$.

to bend the microtubule as is depicted in Figure 4.14. The bending energy of a microtubule is calculated as follows:

$$
E_{\text {bending }}=\int \frac{1}{2} \kappa_{M T}\left(\frac{d r}{d s}\right)^{2} d s=\frac{1}{2} \kappa_{M T} \frac{1}{R^{2}} L,
$$

where $\kappa_{M T}$ denotes the bending stiffness of the microtubule, $\mathrm{R}$ the radius of the contour and $\mathrm{L}$ the length of the microtubule. We measured a radius of $20.5 \mu \mathrm{m}$ and a length of $11.6 \mu \mathrm{m}$ in this experiment. Assuming a bending stiffness of $10^{-23} \mathrm{Nm}^{2}$ (according to Mickey et al. underestimating the bending stiffness of a microtubule [104]) we calculated a bending energy of $\sim 34 \mathrm{k}_{\mathrm{B}} \mathrm{T}$. This value is well above the available thermal energy, eliminating thermal fluctuations as an explanation for the observed motion. The relative-sliding assays therefore support our finding that Cin8 $\Delta$ tail is less efficient in capturing and crosslinking two microtubules than the wild type Cin8, while still being able to displace two microtubules relative to each other. 


\section{$4.5 \quad$ Summary}

In this work, we demonstrate that the removal of the tail domains in the Kinesin- 5 Cin8 dramatically changed its motile properties. When the tails of Cin8 were missing, either in the dimeric chimera - lacking stalk and tails - or in the tetrameric variant in which only the tails are removed, the mechanism that led to a stable regulation of the directionality was disrupted. Furthermore, we showed that the motor variants used in this study exhibited distinct bidirectional qualities. Both constructs moved randomly toward either the plus or minus end of the microtubule. The dimeric chimera Cin8Kin moved along a microtubule in a back-and-forth motion in which the sequence of forward and backward steps seemed to be random. We therefore conclude that the motility-producing subunit of Cin8, i.e. the motor domains and neck linker, are intrinsically bidirectional. The bidirectionality of Cin8Kin as well as Cin8 $\Delta$ tail is consistent with previous reports that individual Cin8 molecules can, in a well defined way, switch from motion toward the plus end of a microtubule to minus-end directed motion [46, 47] and contradicts the notion that this process requires the coupling of multiple motors [45]. Only the cooperation of the motor domains with other elements in the tetrameric protein (e.g. the tail domains) gives rise to a directed mode of movement.

The single-molecule fluorescence assays are supported by load-free single-bead assays. In these assays, Cin8Kin proved to be able to move processively toward both ends of a microtubule. In a loaded conformation in an optical-trapping assay, where the trap exerted a certain force on the motor, we only observed motor activity at very low trap stiffnesses. Surprisingly, the distance Cin8Kin moved until it changed its direction in a loaded assay was significantly reduced compared to a single-molecule assay. Moreover, this distance could be directly converted to a force acting on the motor that was, on average, less than $0.5 \mathrm{pN}$. From our results, we conclude that forces of $\sim 0.5 \mathrm{pN}$ promote a switch of direction in Cin8Kin. This suggests that the regulation of direction might be sensitive to mechanical forces. If this also applies to wild type Cin8, it indicates a mechanism which could be involved in the regulation of forces needed for the function of the mitotic spindle.

In addition to the loss of directional regulation, the tailless tetrameric construct Cin8 $\Delta$ tail showed a drastically reduced ability to crosslink and slide apart two microtubules compared to wild type Cin8. The tail domains therefore not only mediate the directional switching in Cin8 molecules, but also influence the binding of the motor to microtubules. Whether this is due to a high affinity for microtubules of the tail domains themselves, or an indication of a more complex regulation of the motor, remains to be seen. Nevertheless, the fact that the tetrameric Cin8 $\Delta$ tail motor but not the chimeric Cin8Kin was affected by a change of the ionic strength of the buffer solution argues in favor of the latter.

Our data demonstrates that the motile properties of the tetrameric Cin8 $\Delta$ tail were substantially different from those of the dimeric Cin8Kin. While Cin8Kin erratically switched its direction, the Cin $8 \Delta$ tail variant processively moved toward either the plus or minus end, without changing its direction during individual runs. 
In addition, Cin8 $\Delta$ tail was highly processive, with microtubule-interaction times considerably longer than those of Cin8Kin. These results indicate that the architecture of the tetrameric complex, and not merely the presence or absence of the tails, is important in regulating the motile properties of Cin8.

It is very interesting that at least the velocity distribution of Cin8 8 tail and the motile characteristics of Cin8Kin closely resemble what was recently reported for the second S. cerevisiae Kinesin-5 homolog Kip1 [48]. It is tempting to speculate that there is an underlying feature in the yeast Kinesin- 5 motors that enables bidirectional processivity. The deletion of the loop 8 in Cin8, while not fundamentally altering its mode of movement, does influence its motility [46]. Together with the loss of stable directionality upon removal of the tail domains that we observed in the present study, this suggests that a complex interplay of the unique elements of the motility-generating subunits with the tail domains gives rise to the special features of Cin8. We believe that we have identified one important element in the Cin8 protein that is responsible for its ability to processively move toward the minus end of a microtubule. 



\section{In vitro studies of mitotic Kinesin motors in their native environment}

The switching of direction of movement in Kinesin-5 Cin8 motors, elaborated in Chapter 4, is a fascinating example of regulation of mitotic Kinesins. From relativesliding assays, it is obvious that the binding geometry, i.e. if the motor is bound to one or two microtubules, directly influences the directionality [46, 47]. Other members of the Kinesin-5 subfamily as well were reported to change their motile properties when crosslinking two microtubules [62]. To completely understand these regulatory mechanism, it is necessary to study Kinesin-5 motors in an environment that closely resembles the condition of the mitotic spindle. In this chapter, we present a novel optical-trapping assay that enables the investigation of the motile properties of mitotic Kinesins. With this assay, we were able to measure previously unobserved behavior of these motors in a loaded conformation.

During cell division, the bipolar mitotic spindle is assembled. This complex machinery consists of microtubules and microtubule-associated mitotic motor proteins, amongst others. It orchestrates the equal segregation of the genetic material into the two daughter cells $[30,57,105]$. In mitosis, the cytoskeletal microtubules are rearranged to form the bipolar spindle. The formation and maintenance of this spindle is in large parts dependent on several different microtubule-based molecular motors, particularly members of the Kinesin- 5 subfamily. If these motors are disabled, e.g. by the administration of certain drugs, the spindle collapses (see Figure 1.5 on page 9). Cell division is thus impossible and apoptosis inevitable [57, 60].

The assembly of the mitotic spindle requires plus-end directed homotetrameric proteins that are able to processively crosslink the microtubule network. Members

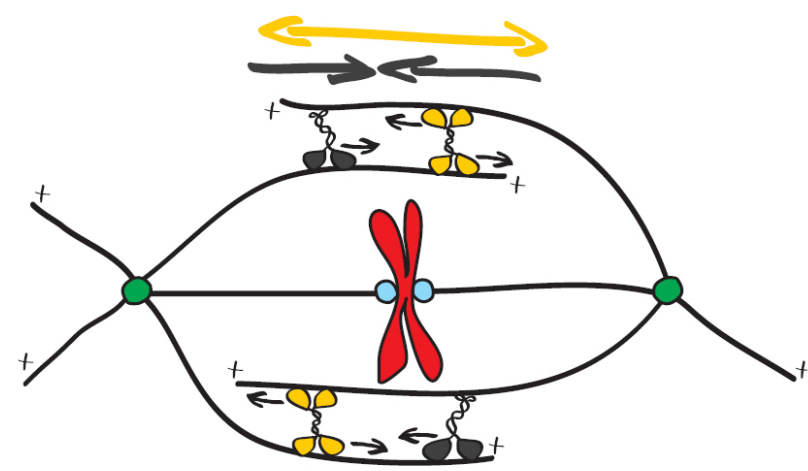

Plus-end motor O Centrosome $\$$ (kinesin-5)

\&o Minus-end motor
Figure 5.1: Simplified sketch of the mitotic spindle

Sketch of a basic model for the mitotic spindle. During mitosis, microtubules, protruding from the centrosomes, overlap to form the mitotic spindle. The microtubules from the two opposite centrosomes are aligned antiparallel. Mitotic motors, e.g. Kinesin- 5 proteins, can bind to two overlapping microtubules to build up forces in the range of several piconewton. This results in segregation of the genetic material. Figure adapted from [9] 
of the widely conserved Kinesin-5 family are well suited for this task, having four identical motor domains arranged in a "dumb-bell" like shape, with two motor domains at each end of a rod (Figure 5.1) [8, 9, 106]. So far, the precise role of Kinesin-5 in spindle morphogenesis remains unknown. One hypothesis for the function of Kinesin-5 is the model of the "push-pull mitotic muscle" in which plusand opposing minus-end directed motors act in the overlap zones of microtubules. Motors can crosslink and slide apart antiparallel microtubules in the spindle midzone using one of two mechanisms: either motors can be built in a bipolar structure with motor domains positioned at both ends (Kinesin-5), or an asymmetric structure that binds one microtubule as cargo (through nucleotide-insensitive microtubule-binding sites) and transports it along neighboring microtubules. Owing to the microtubulepolarity patterns in the spindle, antiparallel microtubule sliding would allow plus-end directed motors to push the poles apart and minus-end directed motors to pull them together (Figure 5.1) [57, 107, 108].

To comprehend the ability of Kinesin-5 motors to generate forces is therefore key to understanding the functional mechanisms of the mitotic spindle. So far, this was predominantly done with single-bead motility assays (Section 2.3) [10]. Unfortunately, binding a Kinesin-5 motor to a microsphere of several hundred nanometers in diameter is an artificial situation. A more physiological approach is used in relativesliding assays (see Section 2.3). It was shown in several of these experiments that tetrameric Kinesin motors are able to push apart antiparallel organized microtubules [9, 47, 62]. In this way, they can recruit additional microtubules into a network and facilitate the subsequent polarization of these bundles. However, relative-sliding assays are performed in a load free conformation, in contrast to the mitotic spindle. We therefore present a novel optical trap assay that offers the opportunity to study mitotic Kinesin motors in vitro under load in an environment closely resembling the conditions in the mitotic spindle.

In addition to studying the motile properties of mitotic molecular motors, this assay allows for the investigation of regulatory processes that might be applied in the mitotic spindle. The delicate balance of forces that is needed to maintain the spindle during mitosis requires carefully tuned interactions of the involved motors with the microtubules. One example is the influence of the binding geometry on tetrameric motors (described in Chapter 4 for Cin8). Furthermore, it might be possible that the angle between two filaments crosslinked by a mitotic motor alters the motor's properties, making certain orientations more favorable than others. It was shown by Yanagida et al. that this is indeed the case for Myosin motors and actin filaments [109]. In contrast, Shingyoji et al. reported that in an optical trapping assay, where they positioned a microtubule attached to a single bead across a doublet microtubule ${ }^{1}$, Dynein motors seemed to be insensitive to filament angles [111]. While the average motile properties of individual motors did not change, the maximum generated force was dependent on the angle between the filaments. They attributed this to the change of the size of the overlap zone between both filaments due to different angles.

The experiments we present here offer the possibility to introduce arbitrary angles

${ }^{1}(9+1)$ microtubule doublets form the structure of an axonemes. Treatment of axonemes with elastase can cause disintegration of the axoneme into individual doublet microtubules [110]. 
between two microtubule filaments and thus allow , for the first time, to systematically investigate the dependence of the motility of mitotic Kinesins on this angle.

\section{The suspended-microtubule assay}

To be able to investigate the motile properties of Kinesin- 5 motors between two filaments in a loaded conformation, we developed a new dual beam optical trap assay (the setup is described in Section 2.2). We used micro pillars to raise a surface-immobilized filament to be able to position an additional filament, that is held into position by two optical traps, on top of it (Figure 5.2). Tetrameric mitotic Kinesin motors can now bind to both filaments and start to push them apart. The use of micro pillars allows us to freely adjust the angle between both filaments. An additional benefit of this experiments is that the motor proteins are far away from the trapping beams. In contrast to conventional single-bead assays, this prevents the occurrence of any photo-damage on the motor proteins. A comparable experiment was done by Yanagida et al. for actin filaments and Myosin motors [109], but to our knowledge this technique was never applied to microtubule-based motor proteins so far.

Carboxylated silica beads with a diameter of $1.5 \mu \mathrm{m}$ (Kisker Biotech GmbH \& Co. KG, Steinfurt, Germany) were first purified by centrifugation ( $5 \mathrm{~min}, 7800 \mathrm{~g}$ )

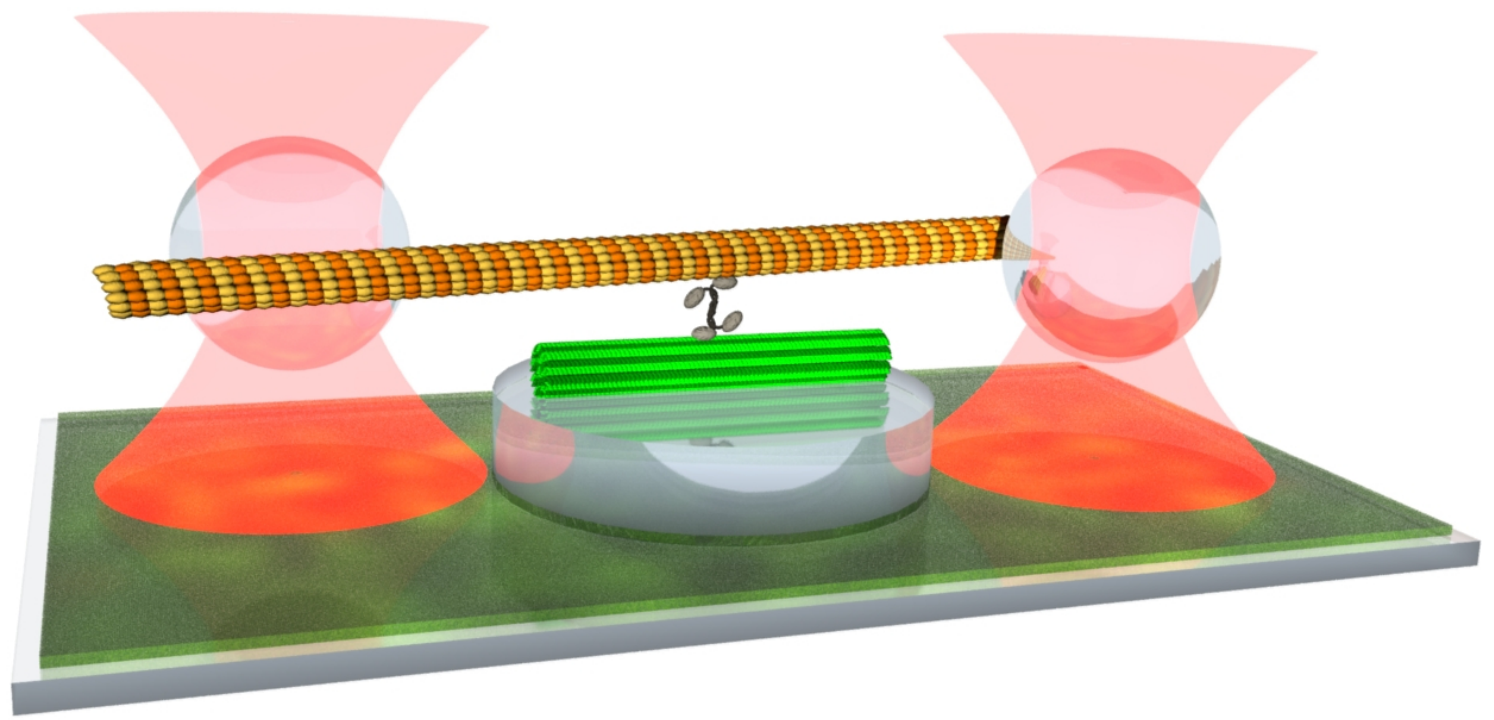

Figure 5.2: Suspended-microtubule assay

A biotinylated microtubule is fixed between two neutravidin coated microspheres which are held in position by an optical trap. This microtubule dumb-bell is antiparallelly aligned with an surfaceimmobilized axoneme. The axoneme is on top of a micro-fabricated pillar on the surface of the measurement chamber. Tetrameric, mitotic motor proteins can bind between both filaments. The motors displace the filaments relative to each other which leads to a built up of forces. Since the axoneme is immobilized on the surface, the motion is transmitted to the microtubule and thus to the beads. The movement of the beads and therefore the forces are measured with the optical trap. 

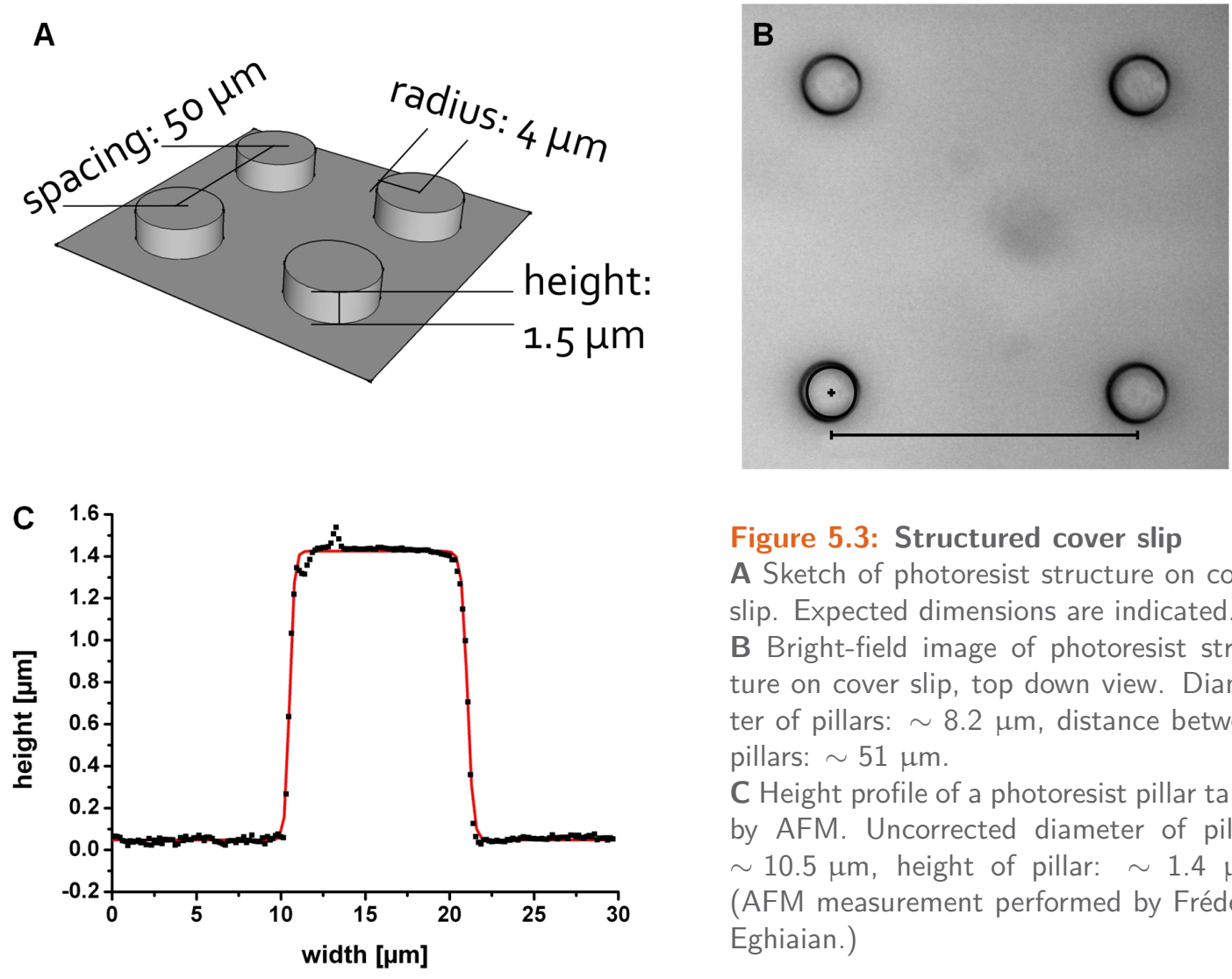

Figure 5.3: Structured cover slip A Sketch of photoresist structure on cover slip. Expected dimensions are indicated. B Bright-field image of photoresist structure on cover slip, top down view. Diameter of pillars: $\sim 8.2 \mu \mathrm{m}$, distance between pillars: $\sim 51 \mu \mathrm{m}$.

C Height profile of a photoresist pillar taken by AFM. Uncorrected diameter of pillar: $\sim 10.5 \mu \mathrm{m}$, height of pillar: $\sim 1.4 \mu \mathrm{m}$. (AFM measurement performed by Frédéric Eghiaian.)

and resuspended in phosphate buffer $\left(50 \mathrm{mM}\right.$ of $\mathrm{KH}_{2} \mathrm{PO}_{4}$ and $\mathrm{K}_{2} \mathrm{HPO}_{4}$ respectively in $\mathrm{H}_{2} \mathrm{O}, \mathrm{pH}$ 7). Incubation for $1 \mathrm{~h}$ with $50 \mu \mathrm{g}$ biotin-xx-cadaverine in the presence of $3.7 \mathrm{mg}$ EDC as a crosslinking agent followed. After an additional purification step, the beads were stored at $4^{\circ} \mathrm{C}$.

The neutravidin coating of the beads was applied shortly before the experiment. $0.5 \mathrm{mg}$ neutravidin and $0.75 \mathrm{mg}$ glycine were added to a $\sim 25 \%$ solution of the biotinylated beads in phosphate buffer. The solution was incubated for $1 \mathrm{~min}$. Afterwards, the excess neutravidin was removed by centrifugation ( $5 \mathrm{~min}, 7800 \mathrm{~g})$ and resuspension of the beads in phosphate buffer. For detailed biochemical protocols see Appendix A.1.

To be able to bring the microtubule attached to the $1.5 \mu \mathrm{m}$ beads close to the surface-immobilized axoneme - without bringing the bead in contact with the cover slip - we used micro pillars fabricated from photoresist (Figure 5.3). For this process, plasma cleaned cover slips were taken into an ISO 5 norm clean room facility. Here, a layer of photoresist (SU8-2001, Michrochem, Newton, MA, USA) with a thickness of $\sim 1.5 \mu \mathrm{m}$ was applied to the cover slips, using a spincoater (OPTIspin SB20, SSE, Singen, Germany). A two-step process was used: first the substrates were spun for $10 \mathrm{~s}$ at $500 \mathrm{rpm}(100 \mathrm{rpm} / \mathrm{s}$ ramp), followed by $30 \mathrm{~s}$ at $1000 \mathrm{rpm}$ (300 rpm/s ramp). The coated cover slips were then soft-baked on a heating plate for $60 \mathrm{~s}$ at $95^{\circ} \mathrm{C}$. Afterwards, the cover slips were exposed to UVlight for $4 \mathrm{~s}$ with a light intensity of $22 \mathrm{~mW} / \mathrm{cm}^{2}$ (using a mask aligner consisting of: Manual High End Mask Aligner MJB4; Constant Intensity Controller CIC1200; Constant Power Controller CPC200/CPC350 Remote, SUSS MicroTec, Berkshire, England) through a photomask (4 inch sodalime glass, evaporated with chromium; 


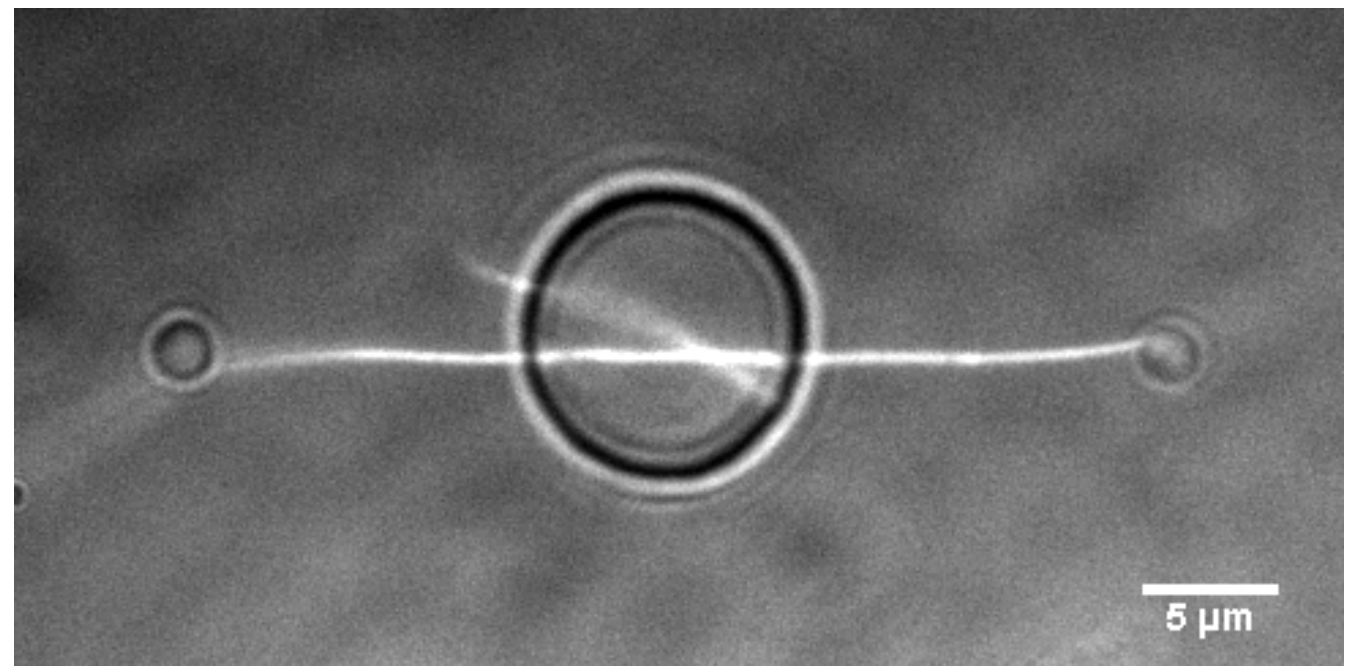

Figure 5.4: Microscope image of suspended-microtubule assay

A photo-resist pillar with an axoneme on its surface is visible. A microtubule dumb-bell consisting of two neutravidin coated beads with a diameter of $1.5 \mu \mathrm{m}$ and a biotinylated microtubule is antiparallelly positioned on top of the axoneme with an angle of $\sim 25^{\circ}$ by a dual-beam optical trap. The microtubule as well as the axoneme is fluorescently labeled with TMR.

ML\&C, Jena-Maua, Germany) containing the positive image of the micro-structure. After the exposure, the cover slips were hard-baked for $120 \mathrm{~s}$ at $95^{\circ} \mathrm{C}$ and developed for $60 \mathrm{~s}$ (using PGMEA developer, Michrochem, Newton, MA, USA). For a more detailed protocol on fabrication see Appendix A.3.

Assay chambers were assembled using micro-structured cover slips and doublesided tape. Fluorescently labeled axonemes were flushed into the sample chamber and allowed to bind to the structure for $5 \mathrm{~min}$. This was followed by $5 \mathrm{~min}$ incubation with $0.1 \mathrm{mg} / \mathrm{ml}$ casein in BRB80 to prevent the beads from sticking to the surface. Afterwards, assay buffer (BRB80 containing $2 \mathrm{mM}$ ATP, $4 \mathrm{mM} \mathrm{MgCl} 2$, and an oxygen scavenging system based on $10 \mathrm{mM}$ DTT, $0.08 \mathrm{mg} / \mathrm{ml}$ catalase $40,0.1 \mathrm{mg} / \mathrm{ml}$ glucose oxidase and $10 \mathrm{mM}$ glucose) containing $5 \mu \mathrm{l}$ of the neutravidin coated beads, $1 \mu \mathrm{l}$ of fluorescently-labeled biotinylated microtubules and mitotic motor proteins in sufficient dilution, was flushed into the chamber. If not otherwise indicated, all chemicals were obtained from Sigma-Aldrich Chemie Gmbh (Munich, Germany).

In the suspended-microtubule assay, a dual beam optical trap was used (see Section 2.2). In this setup, a $1064 \mathrm{~nm}$ laser was split into two orthogonally polarized beams. Each beam formed an independently steerable trap in the specimen plane (in the following denominated direct or indirect trap, respectively). To reconstruct the geometry of the mitotic spindle, first one bead was brought in contact with a microtubule until a neutravidin-biotin linkage formed. This was repeated with the second bead and the same microtubule. This microtubule dumb-bell was aligned in an antiparallel orientation to one of the surface-immobilized axonemes (Figure 5.2 and 5.4).

The use of axonemes allowed us to forego any surface functionalization since axonemes freely attach to glass-like surfaces. The tetrameric motors included in the assay buffer were allowed to bind to both filaments and start to displace them relative to each other. Since the axoneme was tightly attached to the surface, the 


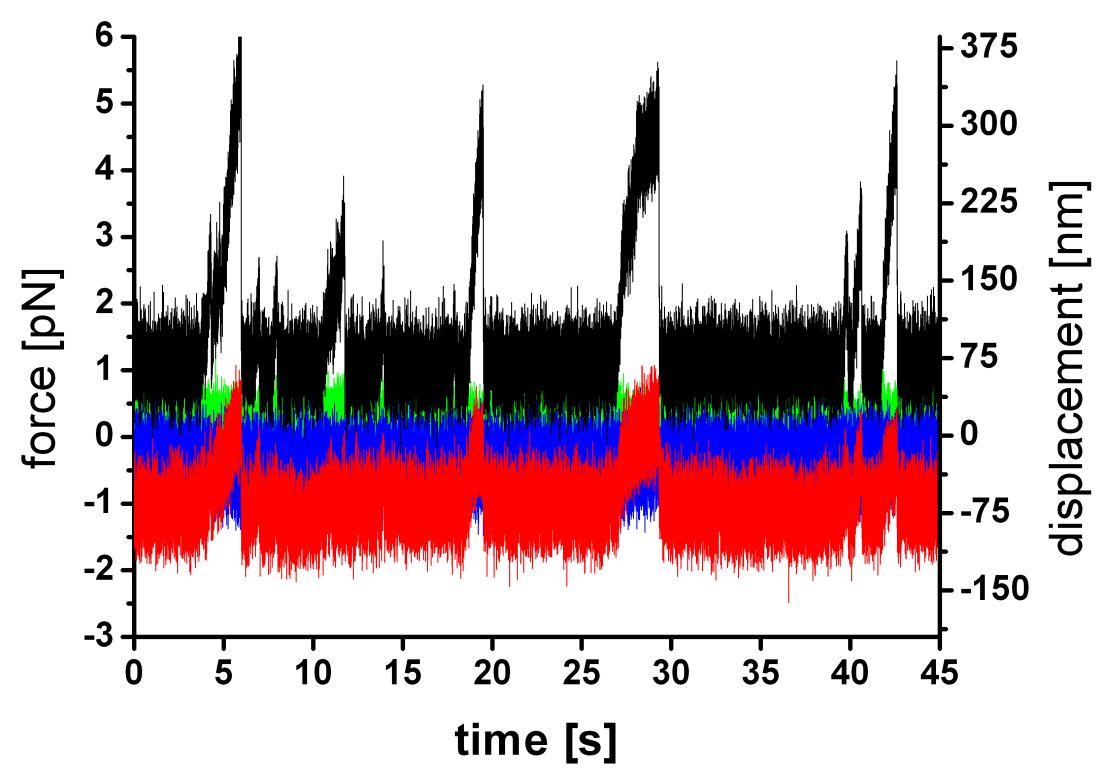

Figure 5.5: Exemplary suspended-microtubule assay data

Raw data from a suspended-microtubule assay with the chimeric motor DK4mer. The $x$ - and $y$-channel of the direct trap are colored black and green; $x$ - and $y$-channel of the indirect trap are colored red and blue. The sum of the offset of both $x$-channels equals the pre-strain of the microtubule dumb-bell. Note that the signal of the indirect $x$-channel decreases until it reaches zero force while the direct $\mathrm{x}$-channel continuously increases toward the maximum force the motor can generate.

complete motion was transmitted to the microtubule and from there to the beads. The movement of the beads was recorded via back-focal plane interferometry [74].

Exemplary data from a suspended-microtubule assay is shown in Figure 5.5. To ensure a tight mechanical coupling, a certain amount of pre-strain was applied to the microtubule dumb-bell. As can be seen from the offset of the two x-channels in Figure 5.5, the pre-strain in this example is in the range of $3 \mathrm{pN}$. During the experiment, one bead was pulled out of the center of the direct trap by the acting motor (black signal in Figure 5.5), while the other was pushed into the indirect trap (red signal in Figure 5.5). When the latter bead reached the focal point of the trap, i.e. a force of $0 \mathrm{pN}$, it was not displaced any further. The microtubule was not stiff enough to push the bead against the potential of the trap and buckled instead. This is evident from the different heights of the signals from the two x-channels in Figure 5.5. For this reason, we only used the data from the bead that is pulled out of one of the traps to calculate the velocity of the motors.

We obtained the unbinding forces by measuring the maximum displacement and converting this into a force using the stiffness of the optical trap. If the pre-strain was sufficient for both beads to experience the same force, we added the signal of both x-channels to get the entire force. Otherwise we only considered the signal of the bead that was pulled out of the trap.

The ability to reposition the two traps in the specimen plane allowed us not only to measure the motile properties of tetrameric proteins between two antiparallel aligned microtubules, but also to introduce an angle between both filaments. In single-bead assays, we can choose a microtubule so that the motors motion is always aligned with either the $\mathrm{x}$ - or $\mathrm{y}$-axis of the setup. In the suspended-microtubule 
assay, even when one filament is aligned with the setup, the introduction of an angle between both filaments resulted in a displacement of the bead that is no longer aligned with one of the axes. This can be seen in Figure 5.10 A on page 73 and more clearly in Figure 5.15 A on page 79. To analyze this motion, we performed a principal component analysis for both traps. The signal of the individual trap was then rotated by the angle calculated from the principal component analysis. This presented us with two signals for each trap, perpendicular or parallel to the motors motion (compare figures 5.10 B and 5.15 B). These signals were analyzed as stated above.

\section{Axoneme}

A variety of cell types, ranging from single cell protozoa and sperm to the ciliated epithelia of the respiratory and reproductive tracts, carry cilia or flagella whose inner core consists of a cytoskeletal structure called the axoneme [113]. Axonemes consist of $9+1$ microtubule doublets (fig 5.6). Between these doublets, multiple molecular motors are situated. Although numerous experiments utilized axonemes without reporting any influence of the motors inside the axoneme on the assay (e.g. $[9,114,115])$, we decided to use axonemes which lack the outer Dynein arm proteins that are located close to the outer surface of the axoneme (Figure 5.6). We cultivated a mutant Chlamydomonas reinhardtii strain (strain CC-2228, Chlamydomonas Resource Center of the University of Minnesota, St. Paul, MN, USA) in TAP medium and isolated and purified axonemes following the methods described in [116]. For details on axoneme isolation and purification see Appendix A.4.

In comparison to microtubules, axonemes offer the advantage of being more rigid, binding more tightly to glass and keeping attached objects farther away from any surface by virtue of their greater diameter. Their tendency to attach to glass, or in our case, photoresist, without any need for surface functionalization made them

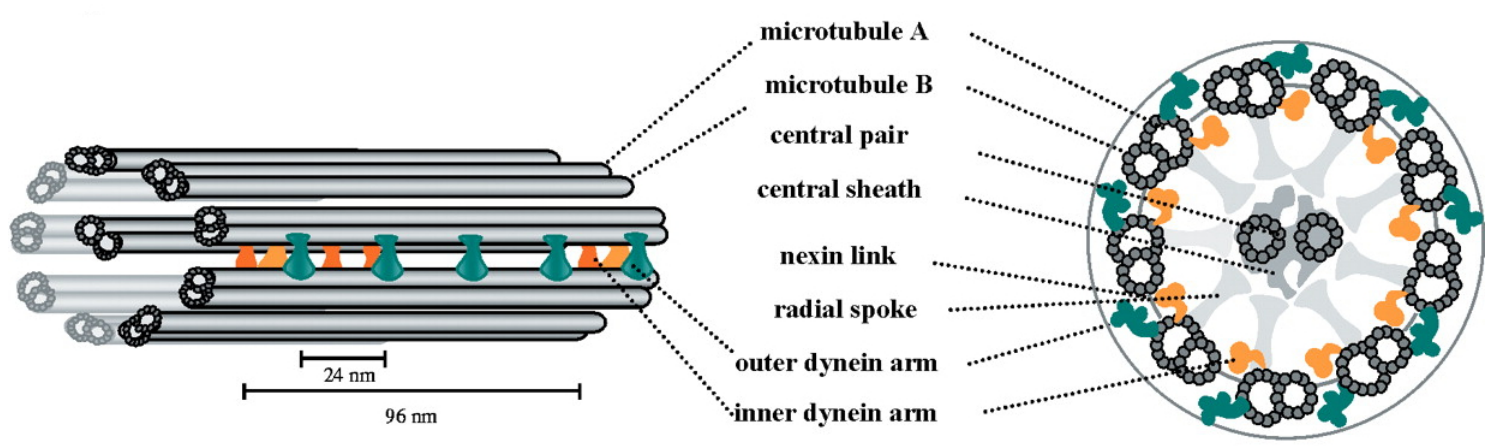

Figure 5.6: Sketch of wild-type axoneme structure

Schematic diagram of an axoneme in length and in cross section. Nine microtubule doublets (microtubules $A$ and $B$ ) surround two central microtubules (central pair). Nexin links, radial spokes and Dynein motor proteins interconnect the microtubules. The Dynein arms are periodically distributed along the axoneme; outer Dynein arms (green) with a $24 \mathrm{~nm}$ periodicity and inner Dynein arms (light and dark orange) with a $96 \mathrm{~nm}$ periodicity. The Dynein arms are multi-protein complexes that project from the A microtubule of every outer doublet; the outer arms (green) face toward the boundary of the axoneme, and the inner arms (orange) face the central sheath. Figure adapted from [112]. 

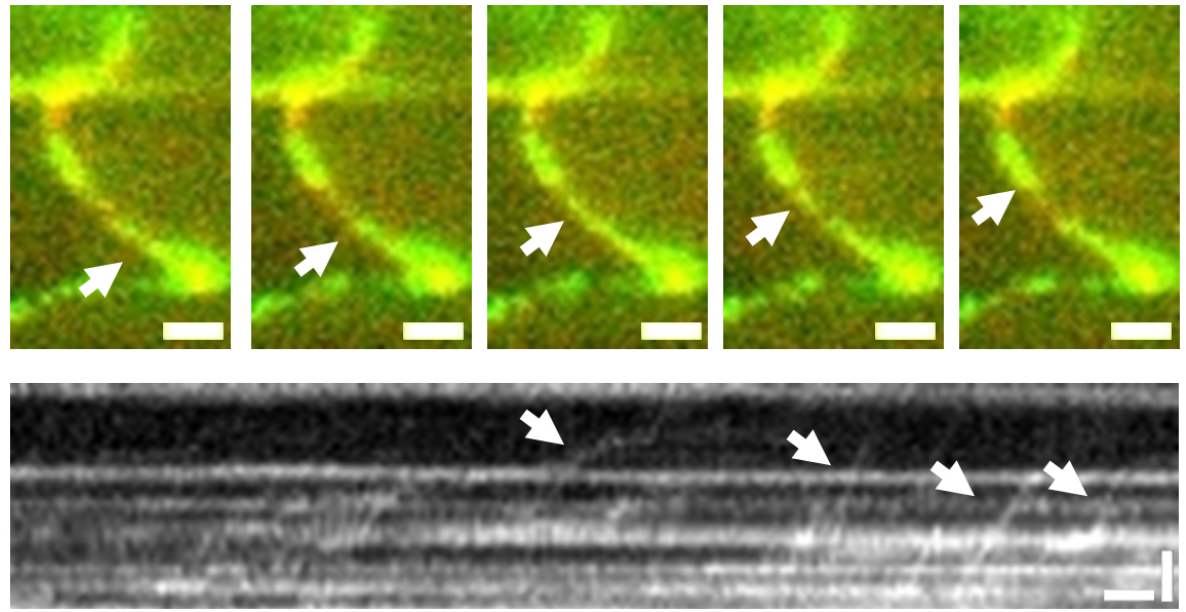

Figure 5.7: Molecular motors on axonemes

Top: Image sequence of a single-molecule fluorescence assay with DK4mer. The arrows mark the position of a GFP-labeled molecular motor (green). Although the tubulin of the axoneme was TMR-labeled (red), strong autofluorescence of the axoneme is visible in green. Scale bar: $2 \mu \mathrm{m}$. Bottom: Kymograph of the assay. The arrows mark motor runs. Vertical scale bars: $3 \mu \mathrm{m}$; horizontal scale bars: $9 \mathrm{~s}$.

ideal for use in the suspended-microtubule assay. With the use of axonemes, we could avoid coating our microstructures with any kind of binding agent. This prevents any problems with the microtubules also binding to the structure and therefore disrupting our experiments.

To test whether molecular motors are able to bind to and move along axonemes, we performed single-molecule fluorescence assays. Since the axonemes during isolation and purification came in contact with the dye of the chloroplasts of the $C$. reinhardtii cells, they show a strong autofluorescence at a wavelength of $\sim 530 \mathrm{~nm}$. Although this interfered with the assay, single molecule motility could be observed (Figure 5.7).

\section{\begin{tabular}{l|l} 
& Chimeric Kinesin-1/Kinesin-5 \\
5.2 & microtubule-sliding motor DK4mer
\end{tabular}}

As a first study with the suspended-microtubule assay, we used the Kinesin-1 head/ Kinesin-5 stalk chimera DK4mer (Figure 5.8). This motor was recently well characterized [69]. On single microtubules it moves with a high average velocity of $\sim 500 \mathrm{~nm} / \mathrm{s}$ and a remarkably long run length of $\sim 9 \mu \mathrm{m}$ [69]. It is able to crosslink and slide apart two antiparallel aligned microtubules. Since DK4mer walks on both microtubules that it crosslinks, the velocity of the moving microtubule should be on average twice the velocity on single microtubules [69] (see Section 1.2).

The data from a suspended-microtubule assay using DK4mer with two antiparallel aligned filaments is shown in Figure 5.9. In a geometry where the filaments are aligned with the $\mathrm{x}$-axis of the setup, the raw data only showed motion of the bead along this axis (Figure 5.9 A). To measure the velocity of the motor, we lin- 
A

Eg5 DK4mer
DMKHC

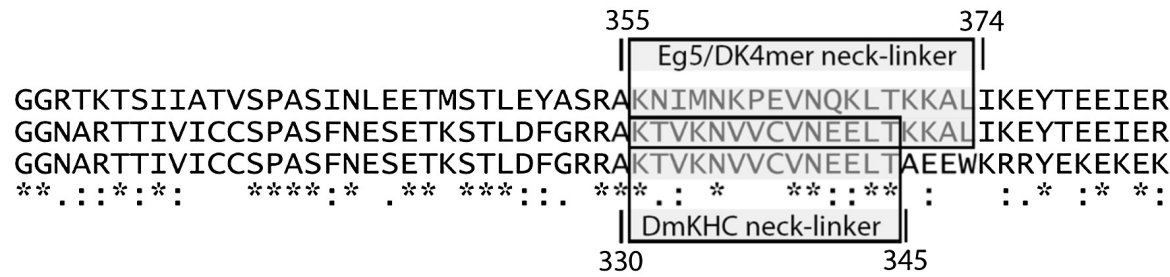

B

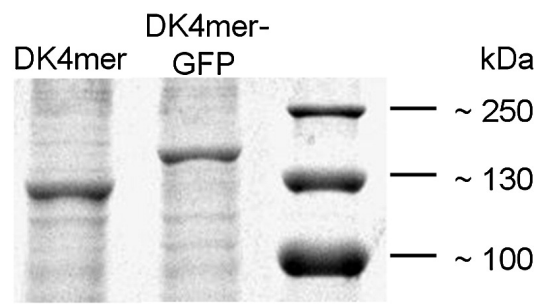

C
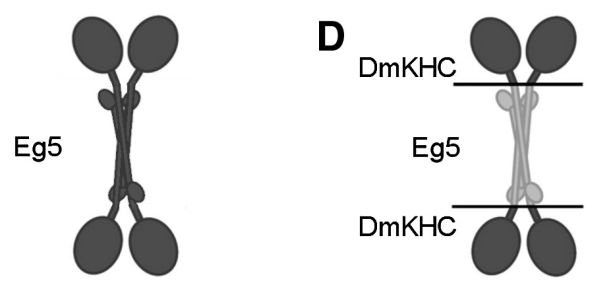

Figure 5.8: Construction of the tetrameric chimera DK4mer

A Details of the junction between the Xenopus laevis Eg5 neck coiled-coil and the Drosophila melanogaster DmKHC motor domain (the numbering refers to the amino acid numbering in the respective wild type motor sequences as indicated by gray boxes). B SDS-PAGE gel showing DK4mer at $130 \mathrm{kDa}$ and DK4mer-GFP at $157 \mathrm{kDa}$ in comparison with molecular weight markers (lane 3). C Cartoon of the overall geometry of the bipolar homotetrameric motor protein Eg5. D Cartoon of the overall geometry of the bipolar homotetrameric chimera DK4mer. Figure adapted from [69].

early fitted the median filtered excursions of the bead from the trap (Figure 5.9 B). To avoid any possible slowdown of the motor due to large forces, we only fitted the initial part of the movement. We obtained an average velocity for DK4mer of $260 \pm 99 \mathrm{~nm} / \mathrm{s}$ (mean $\pm \mathrm{SD}$, Figure 5.11). This is comparable to the velocity on one microtubule but it is significantly slower than the expected value for relative sliding of twice that on a single microtubule. Still, the maximum velocity that was reached in this assay was considerably larger, in the range of up to $900 \mathrm{~nm} / \mathrm{s}$. This is in agreement with typical values achieved in a relative-sliding assay [69].

We calculated the forces from the median filtered signal of the bead that is pulled out of the trap by multiplying it with the trap stiffness (Figure $5.9 \mathrm{~B}$ ). The average unbinding force of Dk4mer was with $4.2 \pm 1.8 \mathrm{pN}$ (Figure 5.11) in the typical range of Kinesin motors but slightly lower than the unbinding forces of Kinesin-1 of $6-7 \mathrm{pN}[7,77]$. Since this was the first measurement of DK4mer forces, there is no comparable data from other experiments. The maximum force in this assay was in excess of $15 \mathrm{pN}$. This might be due to multiple motors binding between the two filaments at the same time. When no angle was applied in the experiment, i.e. complete antiparallel alignment, the overlap zone between both filaments was probably quite large. This increased the probability of multiple motors binding at the same time, and thereby adding up their individual forces. A similar effect was observed for Dynein motors between two filaments [111].

When we introduced an angle between the two filaments in the experiment, the signal of the bead's motion was distributed between the $\mathrm{X}$ - and Y-channel of both traps (Figure $5.10 \mathrm{~A}$ ). A principal component analysis of the channels of both traps provided us with the angle between the two filaments. For the data shown in Figure $5.10 \mathrm{~A}$, the calculated angle was $23^{\circ} \pm 1^{\circ}$, in good agreement with the angle measured from the microscope image $\left(\sim 25^{\circ}\right)$. To get the actual motion along the axis of the microtubule, we rotated the data-set by the calculated angle and applied 
A direct trap
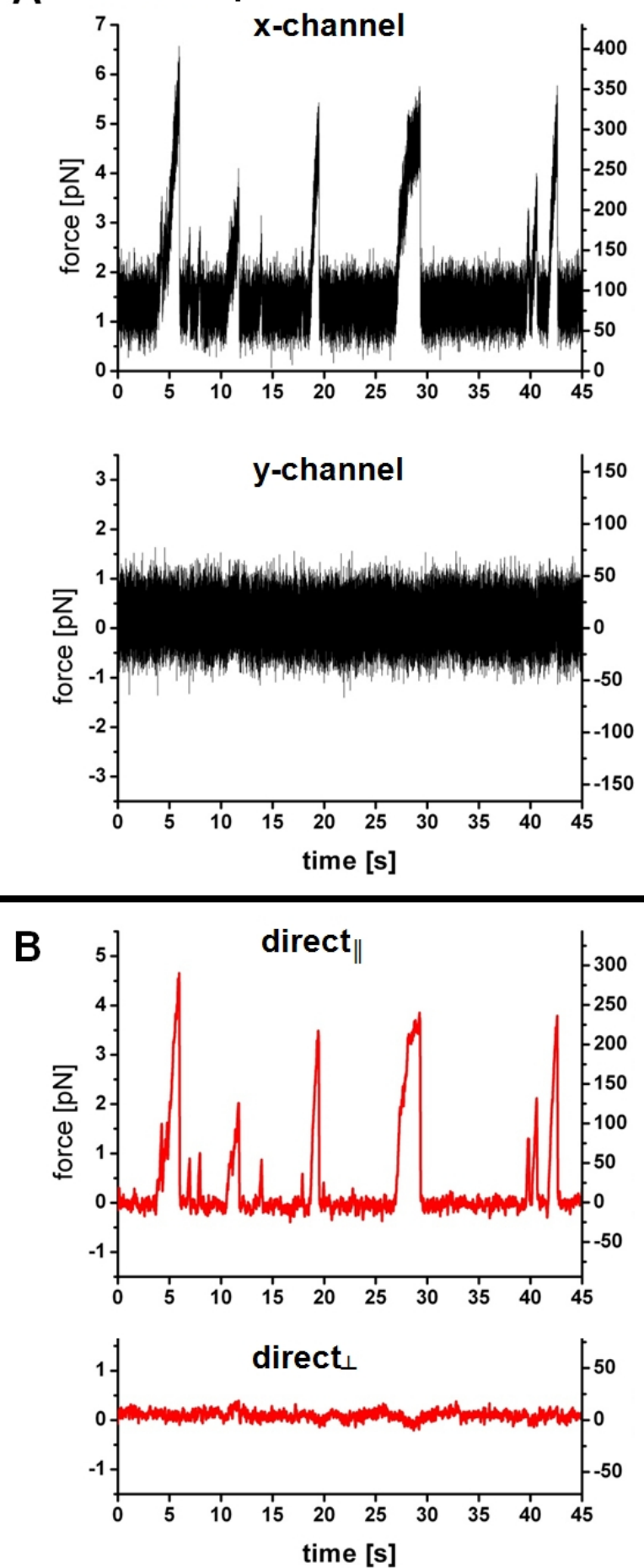

indirect trap
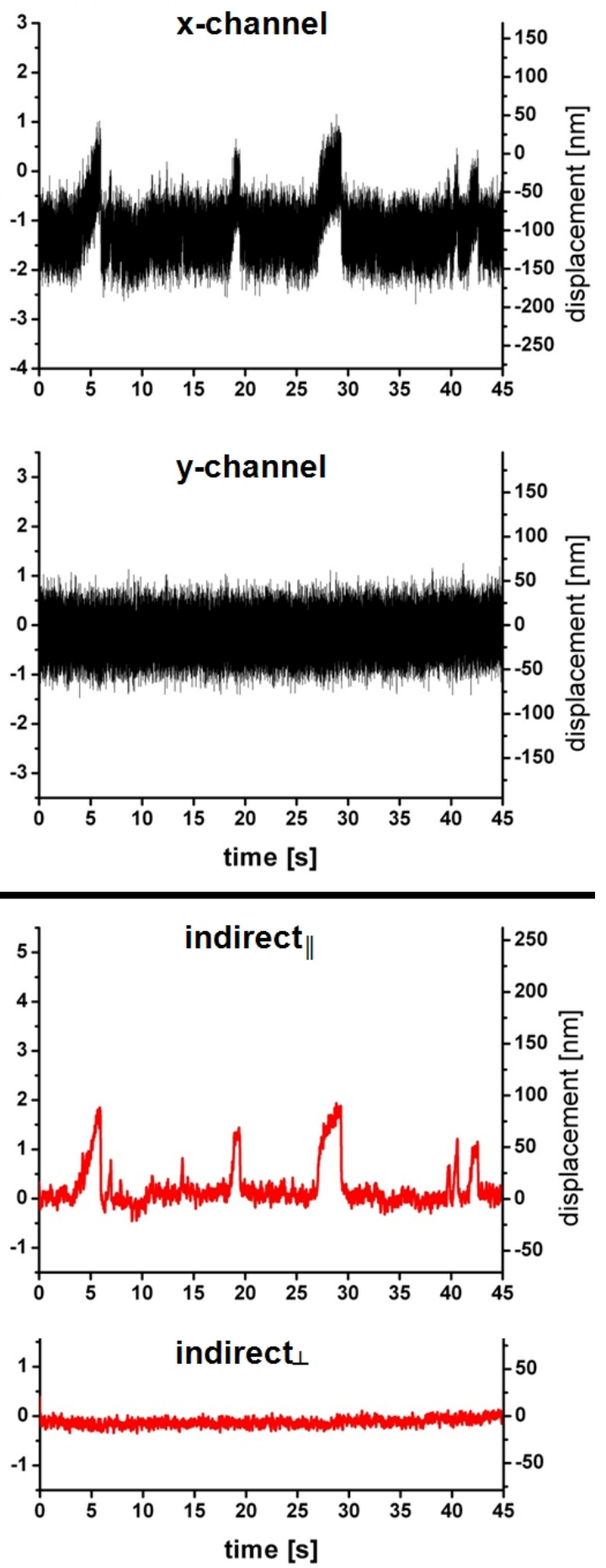

Figure 5.9: Exemplary trapping results for DK4mer at $0^{\circ}$

A Raw data from a suspended-microtubule assay with DK4mer at a filament angle of $0^{\circ}$. Xand $\mathrm{Y}$-channel for both traps are shown. B Processed data, median filtered with $0.03 \mathrm{~s}$ sliding window, from the same experiment. Trap stiffness: $0.018 \mathrm{pN} / \mathrm{nm}$ 
A direct trap
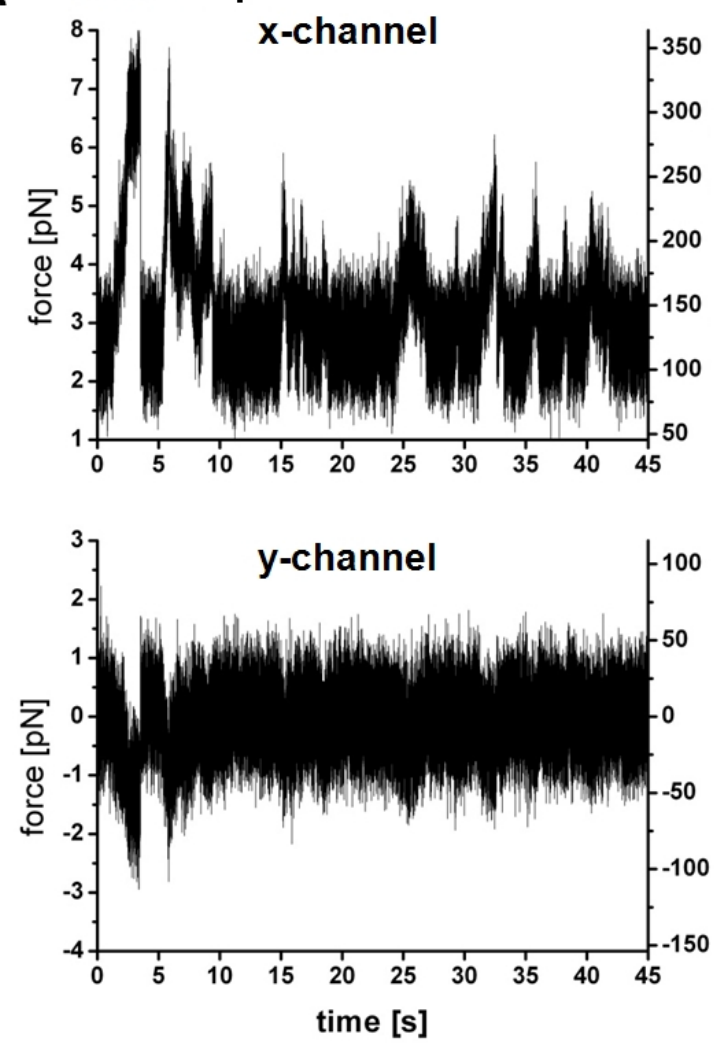

indirect trap
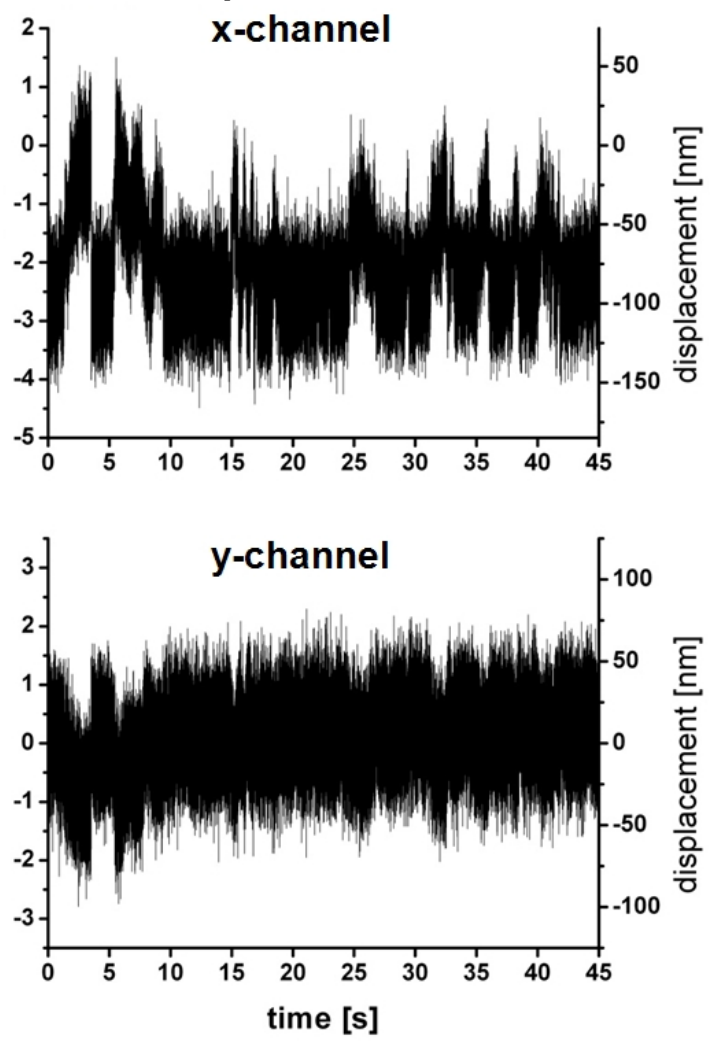
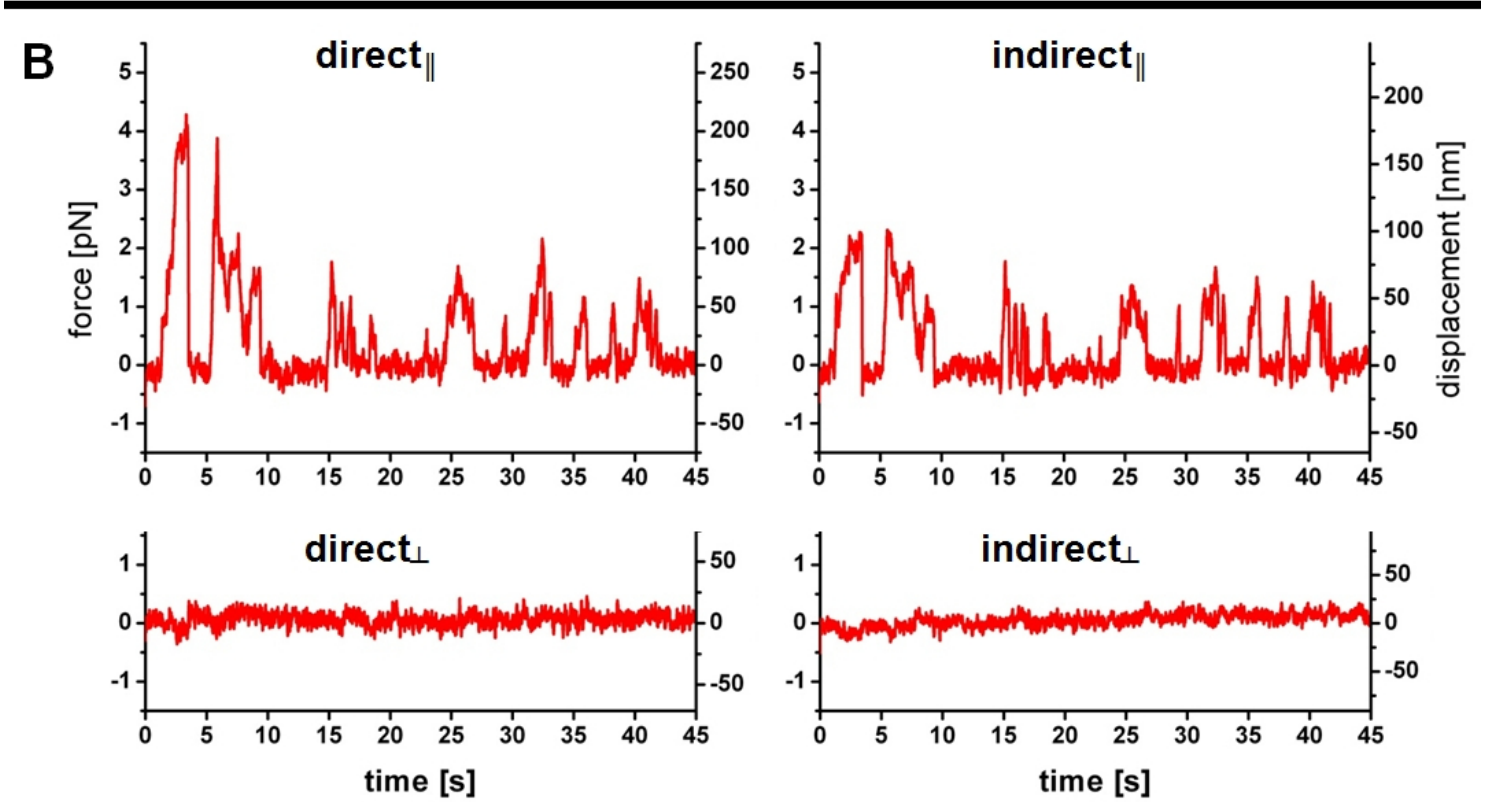

Figure 5.10: Exemplary trapping results for DK4mer at $25^{\circ}$

A Raw data from a suspended-microtubule assay with DK4mer at a filament angle of $25^{\circ}$. Xand Y-channel for both traps are shown. B Processed data from the same experiment. The data-set was rotated by the calculated filament angle to get a signal parallel and perpendicular (denoted by $\|$ and $\perp$ ) to the movement of the motor. The data, median filtered with a $0.03 \mathrm{~s}$ sliding window, is shown. Trap stiffness: $0.026 \mathrm{pN} / \mathrm{nm}$ 


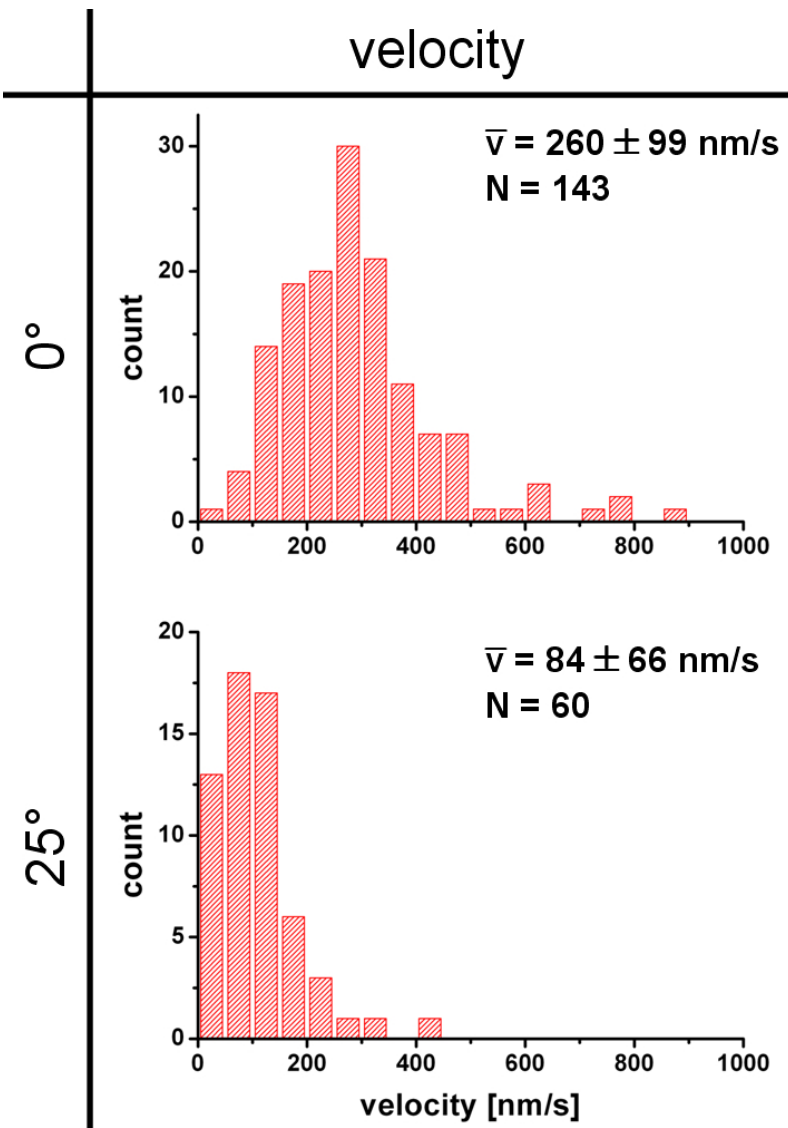

force
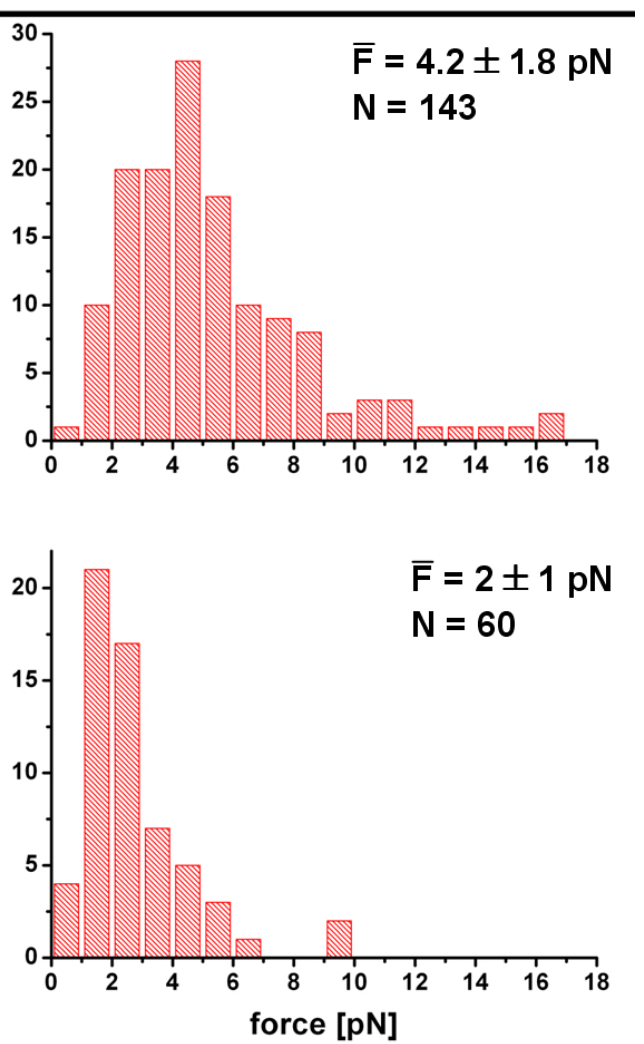

Figure 5.11: Force and velocity analysis for DK4mer

Results from a suspended-microtubule assay with DK4mer. The experiment was performed with two different angles between both filaments, $0^{\circ}$ and $25^{\circ}$. Histograms for velocity and force are shown. N: number of analyzed events.

a median filter (Figure 5.10 B). Similar to the case without a filament angle, we calculated the velocity and force from this signal. Surprisingly, both the velocity and the force drastically decreased (Figure 5.10). The initial velocity was reduced to $84 \pm 66 \mathrm{~nm} / \mathrm{s}$ while the maximum velocity dropped to values below $500 \mathrm{~nm} / \mathrm{s}$ (Figure 5.11). The average unbinding force was $2 \pm 1 \mathrm{pN}$ and the maximum force decreased to below $10 \mathrm{pN}$ (Figure 5.11).

The decrease of the maximum force can be explained by a reduction of the overlap zone due to the introduction of an angle between the filaments. This results in a reduced probability for multiple motors binding to both filaments at the same time, in agreement with previous studies [111]. The reasons for the decrease of the velocity and the average unbinding force are less obvious. While at large angles (approaching $90^{\circ}$ ) it could be possible that we would be unable to resolve the motors' motion along the filament that is not aligned with the line of connection of the two traps, this seems highly unlikely for the small angle of $25^{\circ}$ applied here. One possible explanation can be found in the chimeric structure of the DK4mer protein. Its neck linker has a length of 18 amino acids, similar to native Kinesin-5 motors. It is, however, built up by the 14 amino acid long sequence of the DmKHC neck linker followed by the last four amino acids of the Eg5 neck linker [69]. This design could have made the protein susceptible to torque induced effects. 


\section{Xenopus laevis mitotic Kinesin-5 Eg5}

In a second set of experiments, we used the wild type mitotic Kinesin- 5 Eg 5 from Xenopus laevis (Figure $5.8 \mathrm{C}$ ). In contrast to the DK4mer chimera, Eg5 is a slow molecular motor. The average velocity of Eg5 in single-molecule fluorescence assays is $\sim 20 \mathrm{~nm} / \mathrm{s}$, while it is able to slide apart antiparallel microtubules with a velocity of $\sim 40 \mathrm{~nm} / \mathrm{s}$ - twice that of single molecule speeds [9]. So far, Eg5's capability to generate forces was studied with single-bead assays only [10]. In these experiments, Eg5 proved to be able to build up forces that on average reach $1.6 \mathrm{pN}$ without stalling prior to unbinding, moving with an average initial velocity of $\sim 35 \mathrm{~nm} / \mathrm{s}$ [10] (see Section 1.2).

Interestingly, Eg5 showed a different behavior than DK4mer in the suspendedmicrotubule assay presented here. Exemplary data of an assay with antiparallel aligned filaments is shown in Figure 5.12 A. The velocity obtained from the slope of the median filtered data (Figure $5.12 \mathrm{~B}$ ) had an average value of $22 \pm 13 \mathrm{~nm} / \mathrm{s}$ (Figure 5.16). This is in good agreement with the velocity on single microtubules measured with single-molecule fluorescence assays [9]. As previously discussed in the case of the chimeric DK4mer, the velocity reached a value of twice that speed, as would be expected for this geometry, only on rare occasions.

The force that the motor generated in this experiment was $3.9 \pm 1.4 \mathrm{pN}$ (Figure 5.16). It was significantly larger than the previously reported unbinding force of $1.6 \mathrm{pN}$ in single-bead motility assays [10].

The introduction of small angles between the filament did not influence the motile properties of the Eg5 motor (figures 5.13 and 5.14). The average velocity stayed largely constant $\left(17 \pm 10 \mathrm{~nm} / \mathrm{s}\right.$ for $5^{\circ}, 18 \pm 6 \mathrm{~nm} / \mathrm{s}$ for $15^{\circ}$; Figure 5.16$)$ as did the average unbinding force $\left(3.1 \pm 2.2 \mathrm{pN}\right.$ for $5^{\circ}, 3.0 \pm 1.4 \mathrm{pN}$ for $15^{\circ}$; Figure 5.16). Similar to the observations in the experiments with DK4mer, the maximum forces we measured decreased with increasing filament angle in agreement with the expected reduction in overlap zone of the filaments.

Upon setting the angle between the filaments to a value of $25^{\circ}$ the motion of the bead was evident in the y-channel of the traps as well (Figure $5.15 \mathrm{~A}$ ). We again performed a principal component analysis to convert the data to signals parallel and orthogonal to the bead's motion. The calculated angle was $24^{\circ} \pm 2^{\circ}$ in agreement with the results obtained from microscope images $\left(\sim 25^{\circ}\right)$. From the rotated and median filtered data (Figure $5.15 \mathrm{~B}$ ), we calculated velocity and force as stated above. While we only measured a low number of events, the average velocity of $13 \pm 8 \mathrm{~nm} / \mathrm{s}$ as well as the average unbinding force of $3.6 \pm 1.3 \mathrm{pN}$ is in the error margin of the values obtained for smaller angles (Figure 5.16).

While the maximum force that is maintained in this assay decreased with increasing filament-angle (from $8 \mathrm{pN}$ at $0^{\circ}$ to $5 \mathrm{pN}$ at $25^{\circ}$ ), the average unbinding force as well as the average velocity showed no angular dependencies. This is in contrast to the chimeric DK4mer motor. The angular dependencies of the maximum force can be explained with the increase in the overlap zone between the filaments upon decrease of filament angle. The apparent absence of any dependency on fila- 
A direct trap

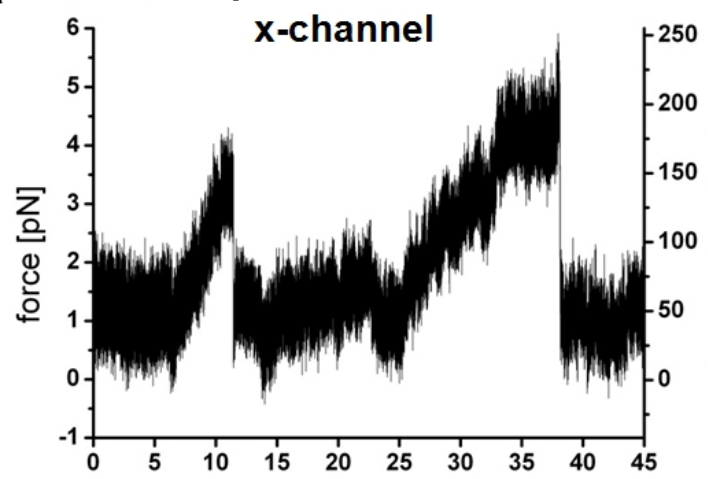

indirect trap
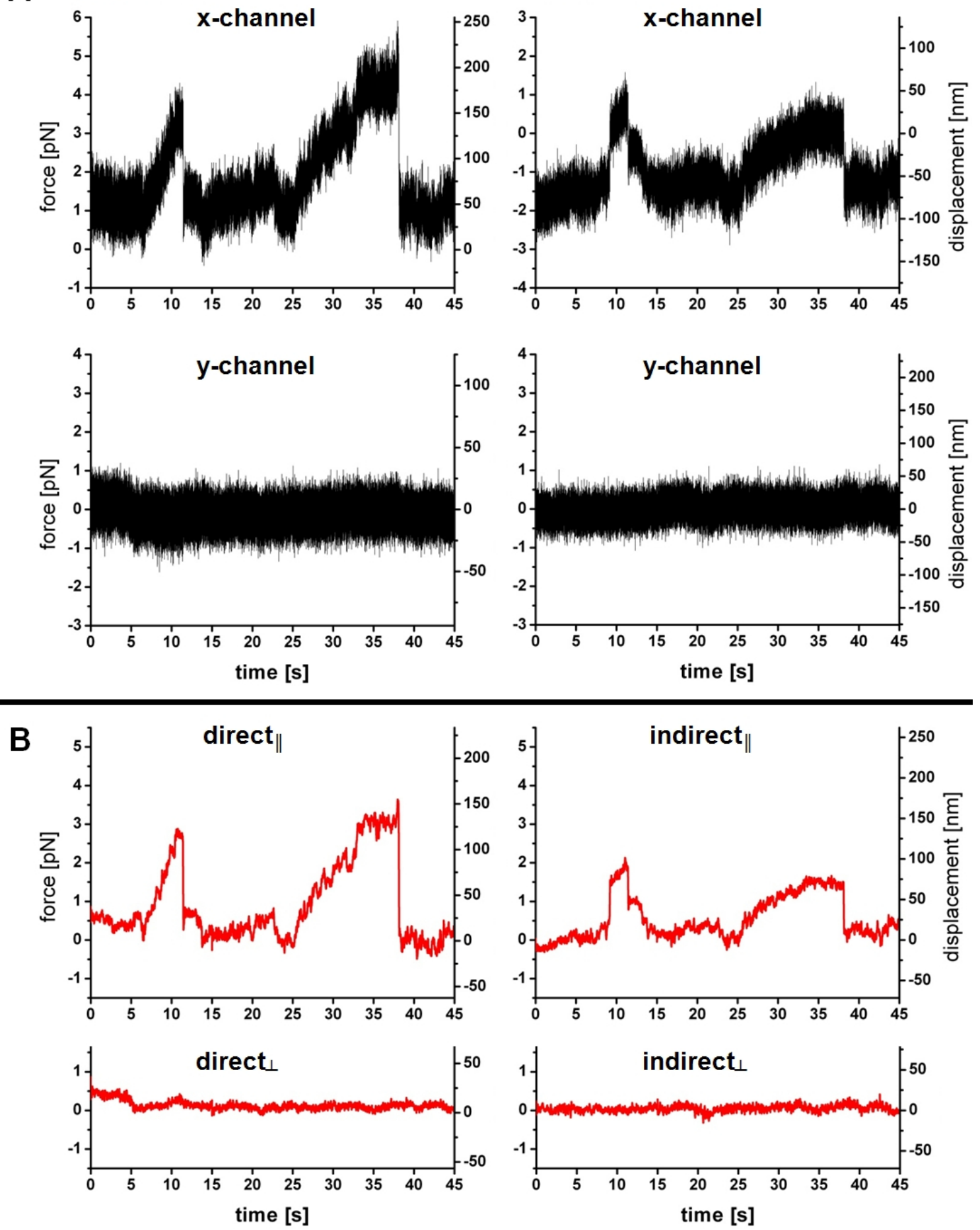

Figure 5.12: Exemplary trapping results for $\operatorname{Eg} 5$ at $0^{\circ}$

A Raw data from a suspended-microtubule assay with $\operatorname{Eg} 5$ at a filament angle of $0^{\circ}$. X- and $\mathrm{Y}$ channel for both traps are shown. B Processed data, median filtered with $0.03 \mathrm{~s}$ sliding window, from the same experiment. Trap stiffness: $0.025 \mathrm{pN} / \mathrm{nm}$ 
A direct trap
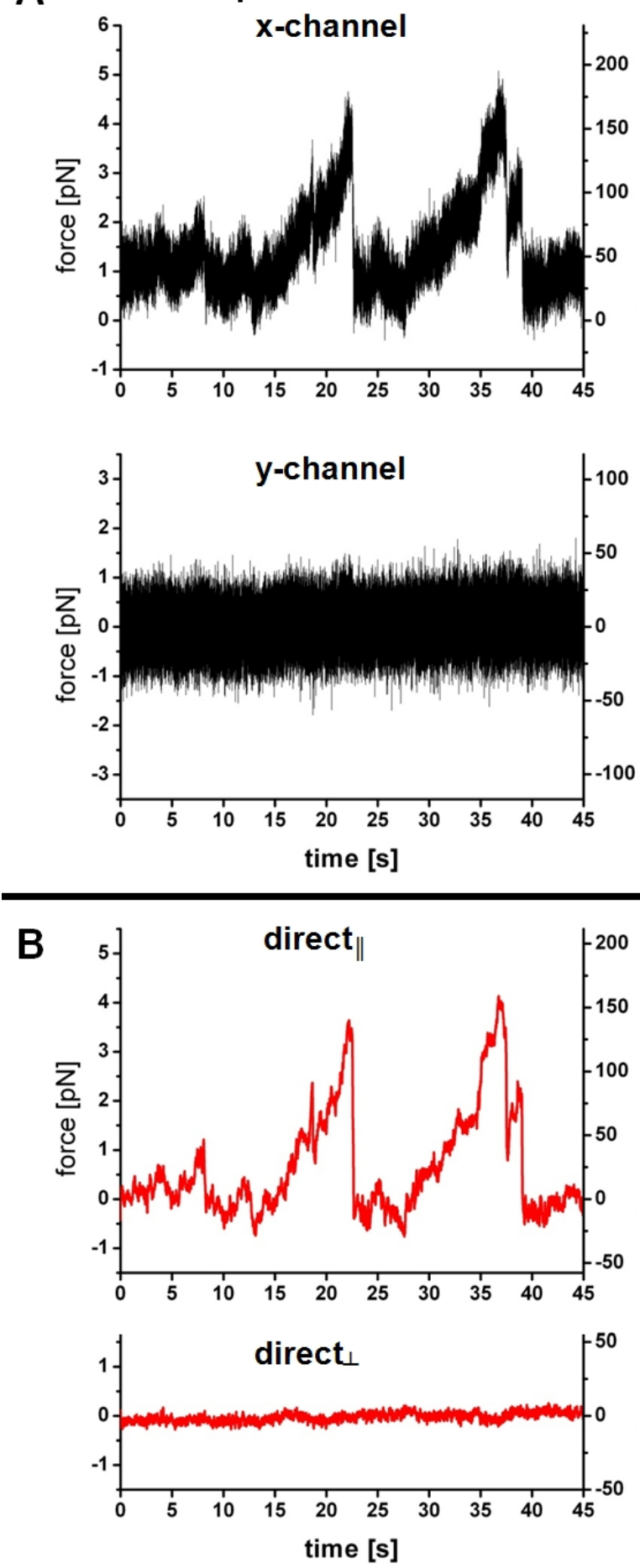

indirect trap
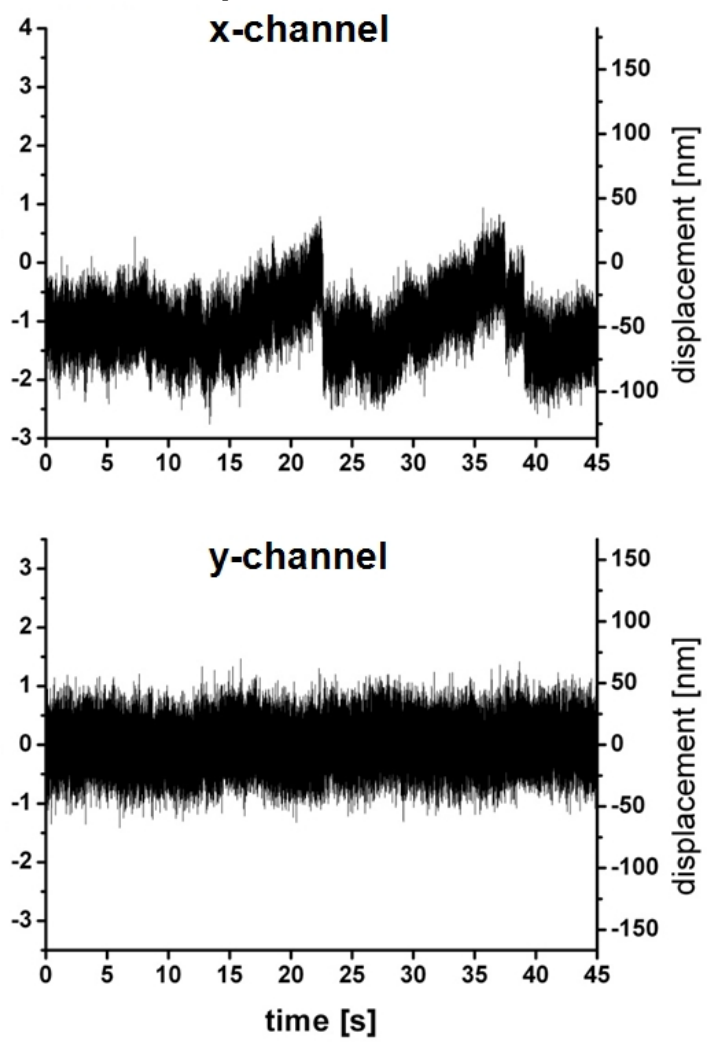

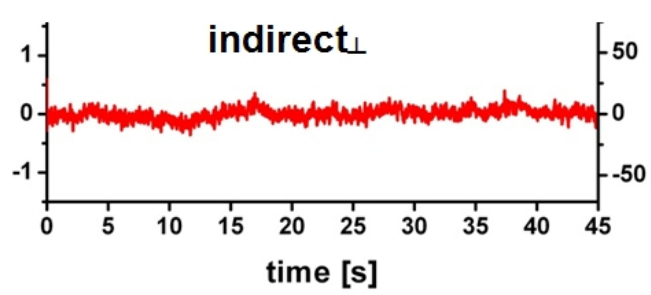

Figure 5.13: Exemplary trapping results for Eg5 at $5^{\circ}$

A Raw data from a suspended-microtubule assay with $\operatorname{Eg} 5$ at a filament angle of $5^{\circ}$. X- and $\mathrm{Y}$ channel for both traps are shown. B Processed data, median filtered with $0.03 \mathrm{~s}$ sliding window, from the same experiment. Trap stiffness: $0.025 \mathrm{pN} / \mathrm{nm}$ 
A direct trap
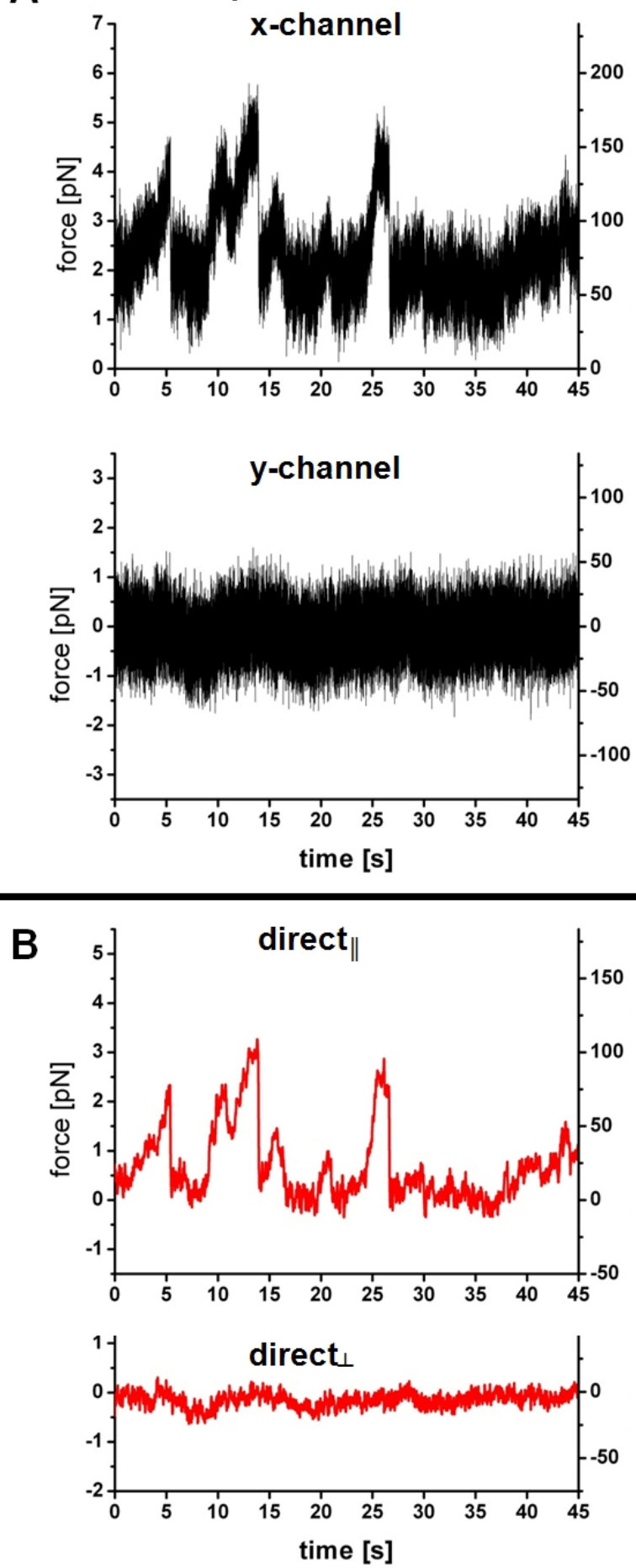

indirect trap
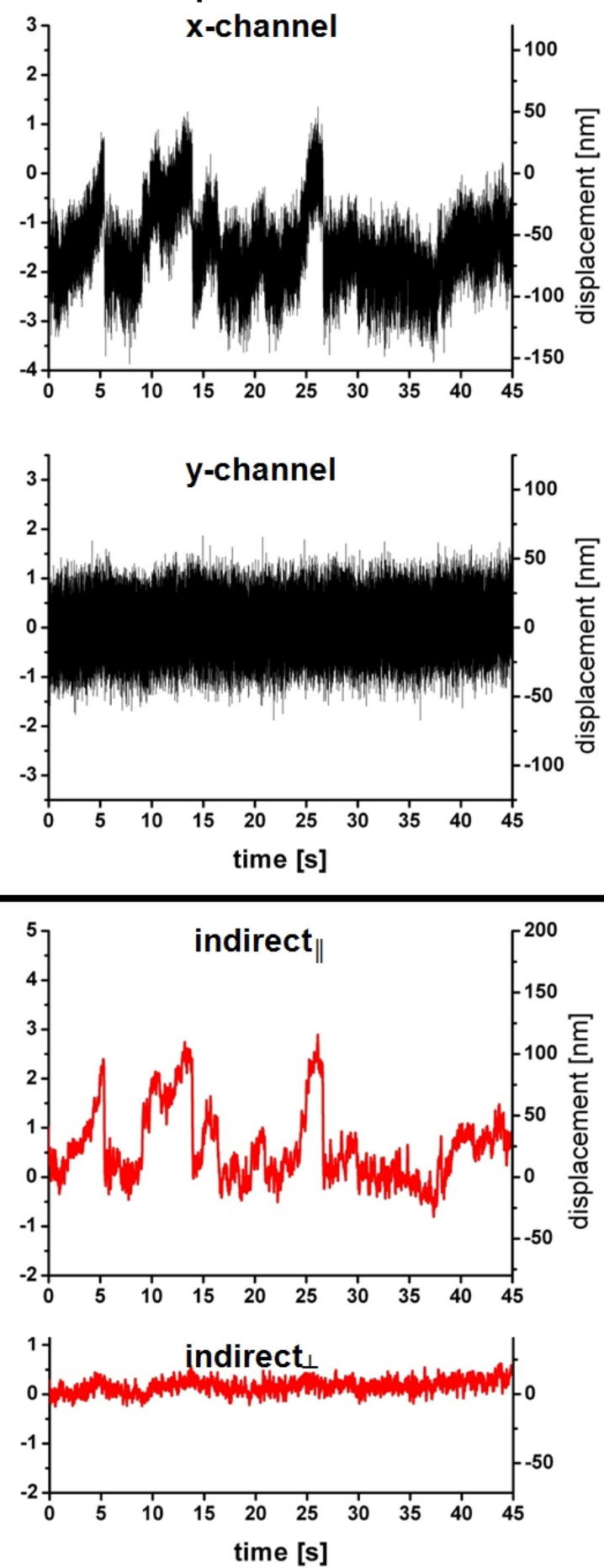

Figure 5.14: Exemplary trapping results for $\operatorname{Eg} 5$ at $15^{\circ}$

A Raw data from a suspended-microtubule assay with $\mathrm{Eg} 5$ at a filament angle of $15^{\circ}$. X- and Ychannel for both traps are shown. B Processed data, median filtered with $0.03 \mathrm{~s}$ sliding window, from the same experiment. Trap stiffness: $0.027 \mathrm{pN} / \mathrm{nm}$ 


\section{A direct trap}
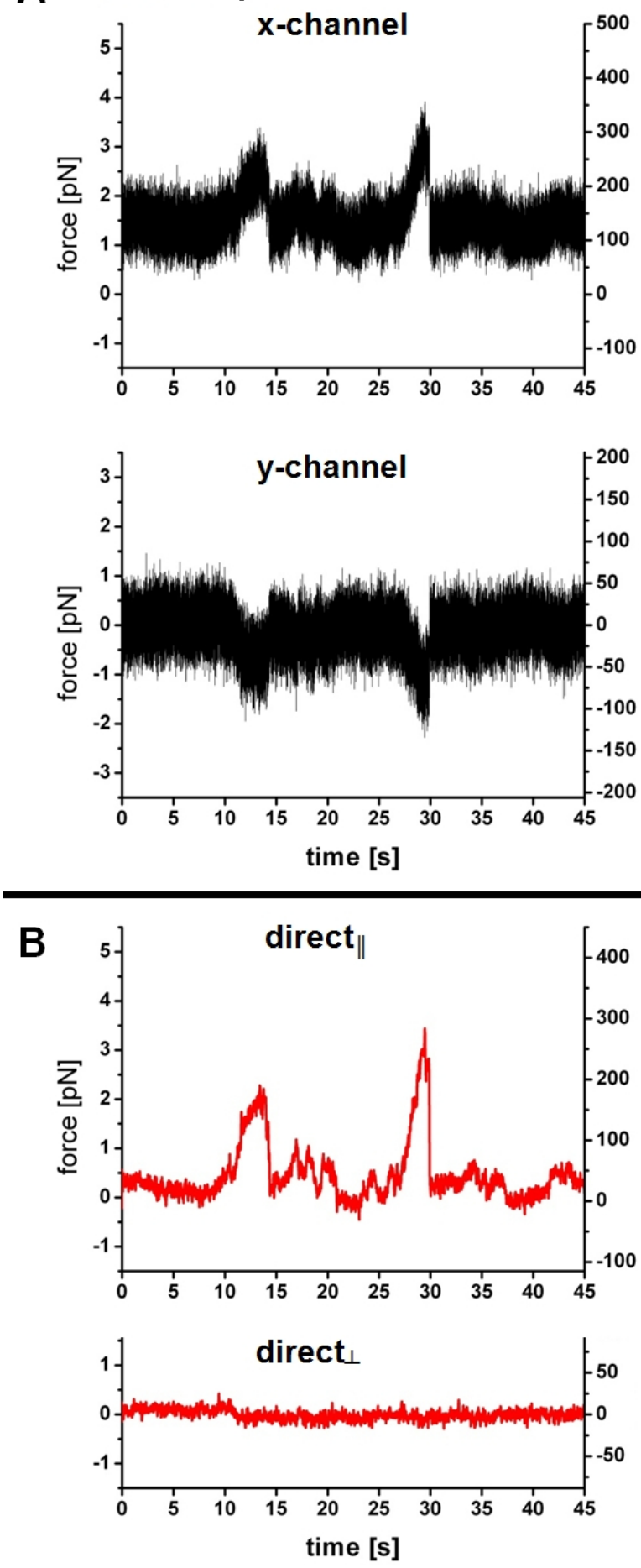

indirect trap
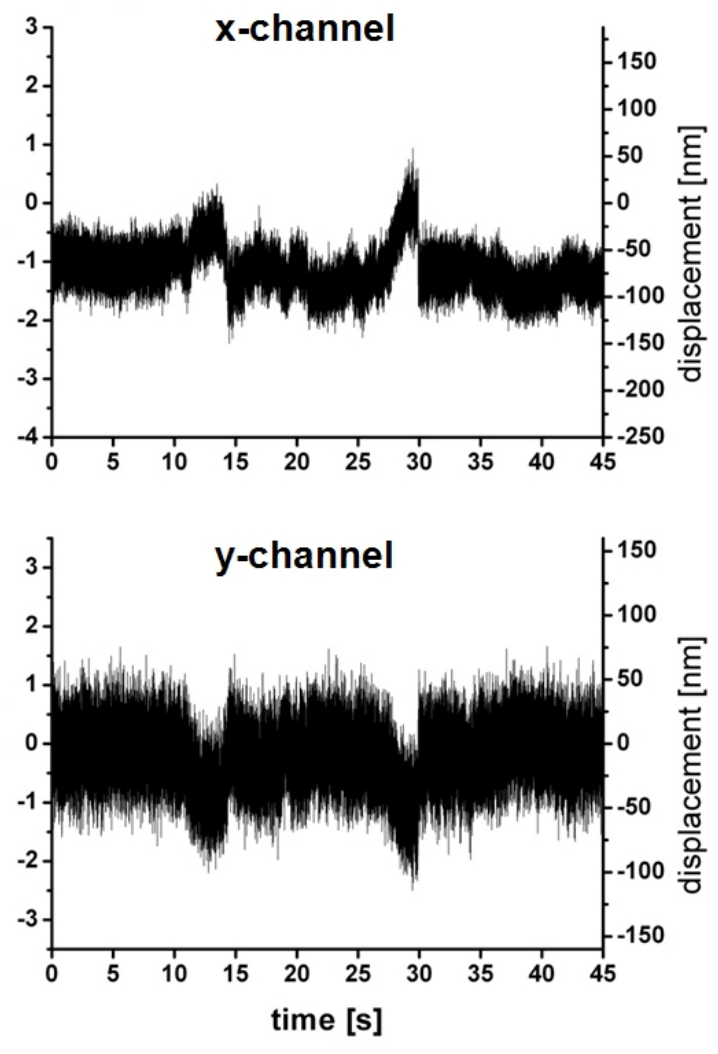

Figure 5.15: Exemplary trapping results for Eg5 at $25^{\circ}$

A Raw data from a suspended-microtubule assay with Eg5 at a filament angle of $25^{\circ}$. X- and Y-channel for both traps are shown. B Processed data from the same experiment. The data-set was rotated by the calculated filament angle to get a signal parallel and perpendicular (denoted by $\|$ and $\perp$ ) to the movement of the motor. The data, median filtered with a $0.03 \mathrm{~s}$ sliding window, is shown. Trap stiffness: $0.017 \mathrm{pN} / \mathrm{nm}$ 


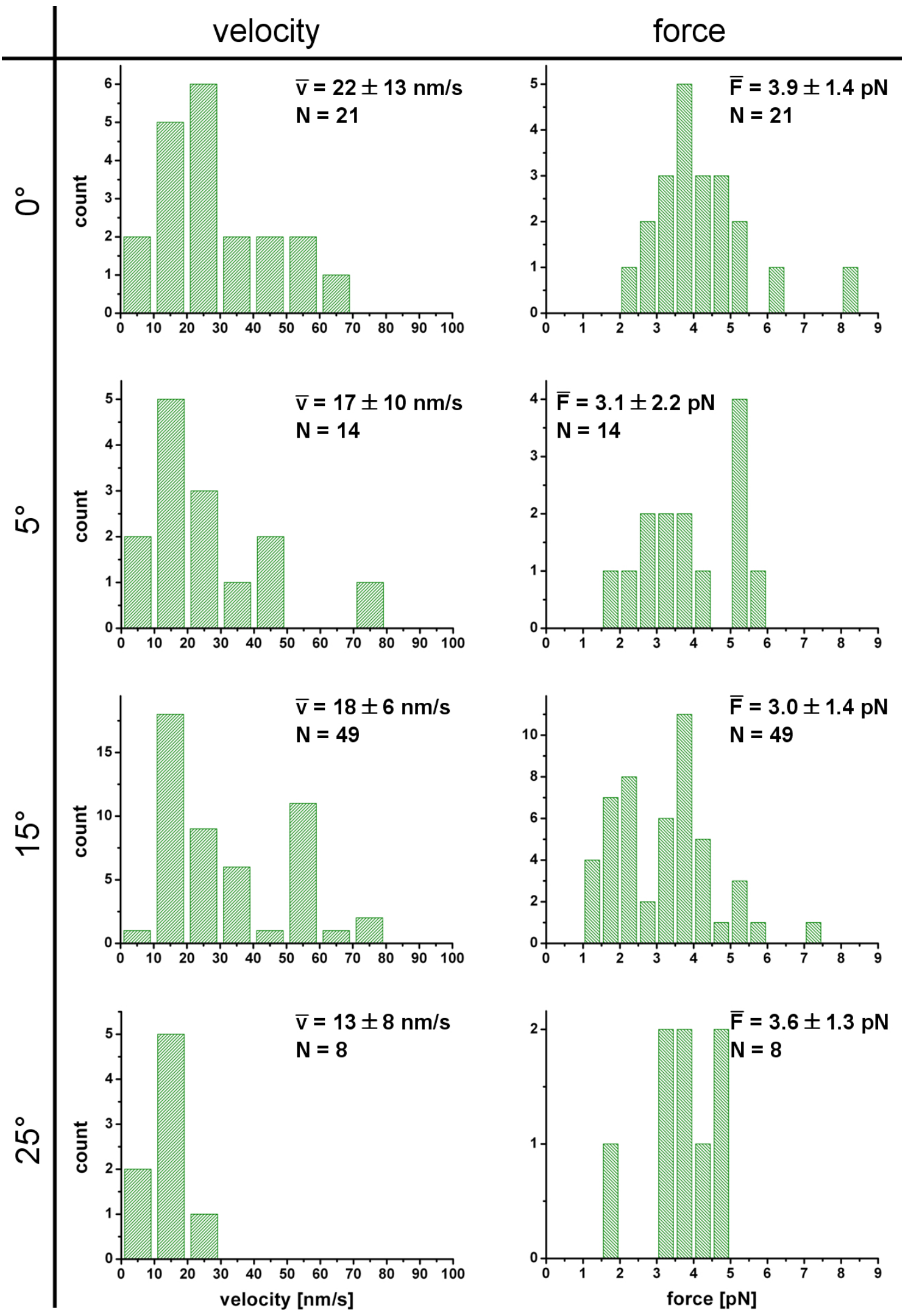

Figure 5.16: Force and velocity analysis for Eg5

Results from a suspended-microtubule assay with Eg5. The experiment was performed with four different angles between both filaments, $0^{\circ}, 5^{\circ}, 15^{\circ}$ and $25^{\circ}$. Histograms for velocity and force are shown. N: number of analyzed events. 
ment angle of the other motile properties might be a result of the adaptation of the wild type Eg5 motor to its role in spindle morphogenesis. Kinesin- 5 motors, having one of the longest neck linker reported for Kinesin proteins, might be insensitive to effects that result from unaligned filaments.

\section{Saccharomyces cerevisiae mitotic Kinesin-5 Cin8}

The Kinesin-5 homologue from Saccharomyces cerevisiae, Cin8, has some outstanding properties (see Chapter 4). This makes Cin8 an ideal object for a study with the new suspended-microtubule assay presented here. Unfortunately, we were unable to produce a Cin8 sample that was sufficiently pure to be used in this assay. Even relatively small contamination with damaged motors or other proteins can result in the breakdown of the assay. If crosslinks form between the filaments that can withstand forces in the range of $10 \mathrm{pN}$, no molecular motor will overcome this linkage. Fortunately, while assembling microtubule dumb-bells, occasionally multiple microtubules would link the two beads (Figure 5.17 A). These bundles, which consisted of an unknown number of microtubules with random orientation, sometimes proved to be contractile. In Figure 5.17 B the data from one of these contractile bundles, formed with Cin8, is shown.

The tetrameric Cin8 motors crosslinking the microtubules contracted the bundle along the line of connection of the two beads. The movement characteristics are similar to the trap traces obtained with the suspended-microtubule assay. We used the median filtered data to calculate the motile properties of Cin8. The average velocity of $24 \pm 7 \mathrm{~nm} / \mathrm{s}(\mathrm{N}=5)$ was considerably lower than what is to be expected from single-molecule fluorescence studies of Cin8 (plus-end directed velocity is on average $\sim 100 \mathrm{~nm} / \mathrm{s}$, see Chapter 4 ). It is possible that multiple motors on microtubules with different alignments obstructed their respective motion which resulted in a reduced velocity. Given that we know neither the number nor the orientation of the microtubules forming the bundle, it is highly unlikely that the measured velocity corresponds to single molecule properties.

The forces we measured were on average $3.1 \pm 1.1 \mathrm{pN}(\mathrm{N}=5)$ and thus reasonable for single Kinesin motors. The same arguments stated above to explain the low velocity make the obtained value for the forces not reliable. Still, these results present, to our knowledge, the first measurements of the forces generated by Cin 8 molecules. It remains to be seen if these findings can be confirmed by future studies with the suspended-microtubule assay. 
A

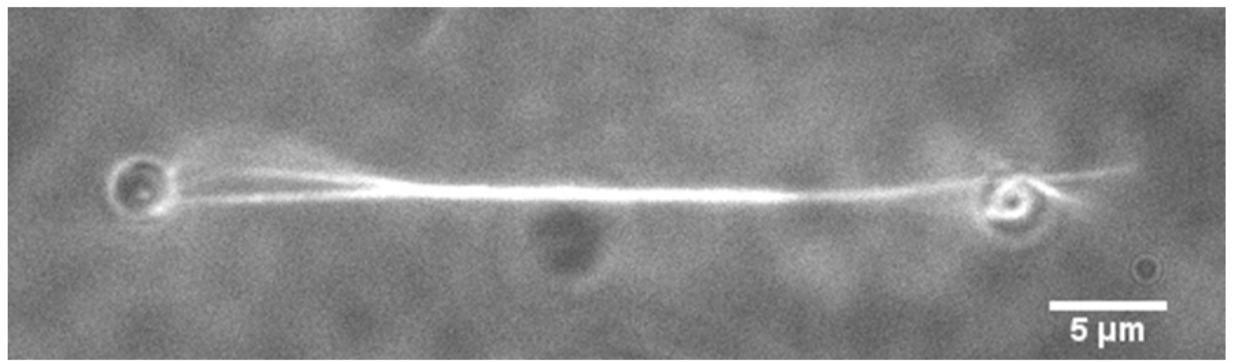

B direct trap
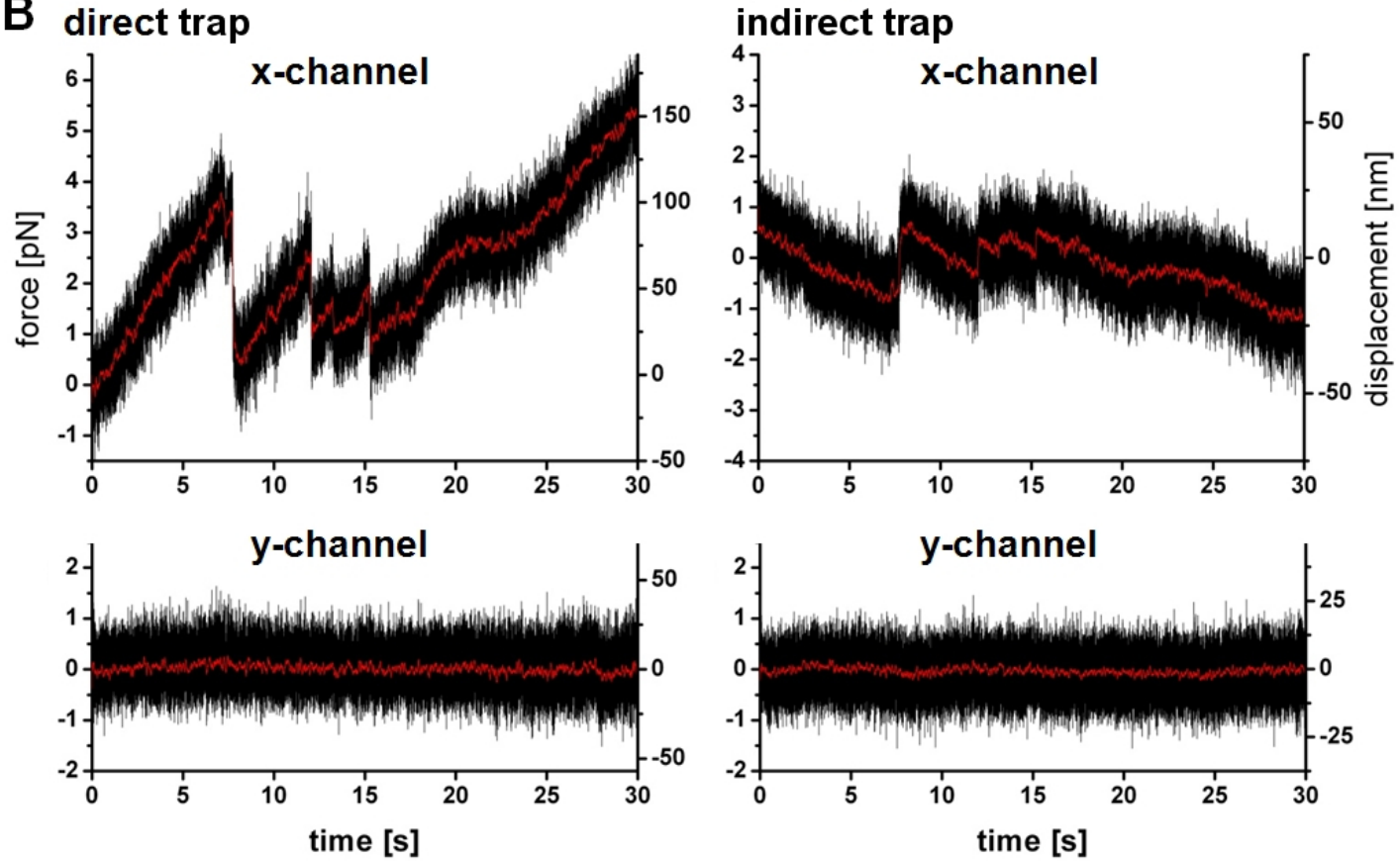

Figure 5.17: Contractile bundle with Cin8

A Fluorescence image of a microtubule dumb-bell. Instead of a single microtubule, multiple microtubules are attached to the trapped beads. Together with crosslinking Cin8 motors a contractile bundle is formed. B Trapping data of the contractile bundle. The raw signal is shown in black, data median filtered with a $0.3 \mathrm{~s}$ sliding window in red.

\section{5 \\ Summary}

To our knowledge, we have presented the first optical trap based assay which is suitable to study mitotic Kinesin motor proteins in a geometry closely resembling the mitotic spindle. We investigated the dependence of the motile properties of two different molecular motors on small filament angles. The results for the fast, chimeric Dk4mer and the slow, wild type Eg5 are summarized in Table 5.1.

The first surprising observation was that, even when the filaments are completely antiparallel aligned, neither of the two motors reached velocities that were reported in relative-sliding assays. However, the velocities measured with the suspendedmicrotubule assay are in good agreement with the velocities of individual motors moving along a single microtubule $[9,62,69]$. This might be due to artifacts induced by the use of axonemes. It is, for example, possible that the Dynein motors that are a part of the axonemes - as well as the Kinesins - interacted with the microtubule 


\begin{tabular}{lcccc|cccc} 
& \multicolumn{4}{c|}{ DK4mer } & \multicolumn{4}{c}{$\operatorname{Eg} 5$} \\
\cline { 2 - 9 } & $0^{\circ}$ & $5^{\circ}$ & $15^{\circ}$ & $25^{\circ}$ & $0^{\circ}$ & $5^{\circ}$ & $15^{\circ}$ & $25^{\circ}$ \\
\hline \hline velocity $[\mathrm{nm}]$ & $260 \pm 99$ & $\mathrm{ND}$ & $\mathrm{ND}$ & $84 \pm 66$ & $22 \pm 13$ & $17 \pm 10$ & $18 \pm 6$ & $13 \pm 8$ \\
force $[\mathrm{pN}]$ & $4.2 \pm 1.8$ & $\mathrm{ND}$ & $\mathrm{ND}$ & $2 \pm 1$ & $3.9 \pm 1.4$ & $3.1 \pm 2.4$ & $3 \pm 1.4$ & $3.6 \pm 1.3$ \\
$\mathrm{~N}$ & 143 & $\mathrm{ND}$ & $\mathrm{ND}$ & 60 & 21 & 14 & 49 & 8 \\
\hline
\end{tabular}

Table 5.1: Table of results for DK4mer and Eg5

all values: mean \pm standard deviation;

$\mathrm{N}$ : number of measured events; ND: measurement not performed.

dumb-bell. Several facts point against this, though. Firstly, axonemes have been used in motility assays in a large number of previous studies without any reported influence on the experiment $[9,114,115]$. Secondly, we used axonemes from a mutant Chlamydomonas reinhardtii strain that lacked the outer-arm Dyneins which are most likely to be able to interfere with our experiment.

Another explanation could be that in a loaded situation, where the crosslinked filaments cannot easily slide apart, the characteristics of the motility of the motor changes. The two adjacent motor domains are no longer independent. Instead, the intramolecular tension built up by the motor itself might lead to a coupled motion. Taking into account both head groups, the probability of unbinding is twice as high when crosslinking two filaments. The tension could lock one head group firmly to the microtubule. Only one side of the protein would thus be able to processively slide apart the filaments, in agreement with our results. The existence of a microtubule binding site in the tail domains of Eg5 [67] could be involved in a mechanism for the protein to "sense" its environment and tightly bind to a microtubule. In the mitotic spindle, this might ensure that individual motors are bound more tightly to the spindle microtubules ensuring maximum efficiency.

The second finding was that, while the native Kinesin-5 seemed to be largely unaffected by the introduction of small angles between both filaments in the assay, the motile properties of the artificial chimera changed drastically (Table 5.1). The global decrease of the maintained maximum force that was observed for both motors can be explained by a change of the size of the overlap zone depending on the filament angle. This, however, does not explain the decrease in velocity and average unbinding force of DK4mer. The velocity dropped well below that of single DK4mer molecules in single-molecule fluorescence assays which cannot be explained by a changing number of motors in the overlap zone in this assay. DK4mer is a highly artificial motor. The hybrid design of the neck linker, which consists of the 14 amino acid long sequence of DmKHC and the last 4 amino acids of the sequence of the Eg5 neck linker, might lack the flexibility needed for efficient microtubule crosslinking. Thus, the native Kinesin-5 may be better suited to fulfill its role in the mitotic spindle. Its molecular structure, e.g. the neck linker, might render it insensitive to any torque between the two head groups of the protein. In spindle morphogenesis, this would be very useful for recruiting microtubules to - and aligning them with the spindle structure. It was shown that Eg5 is able to sort and align microtubules in an antiparallel orientation. The ability to freely twist its head groups might be essential for this. 
A

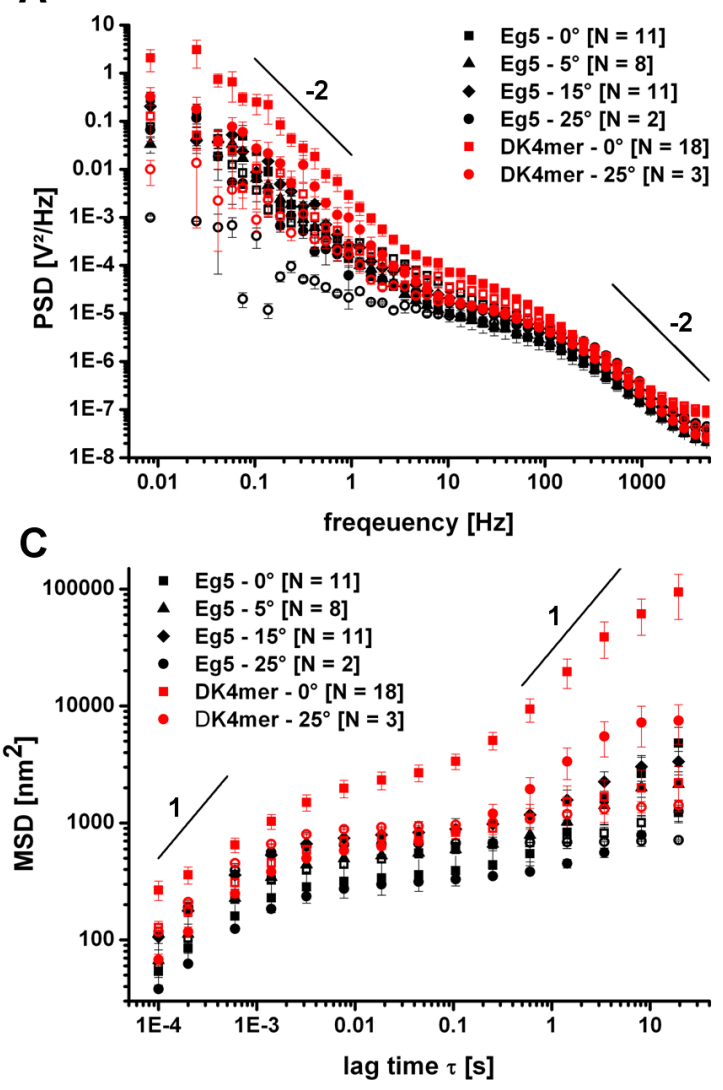

B
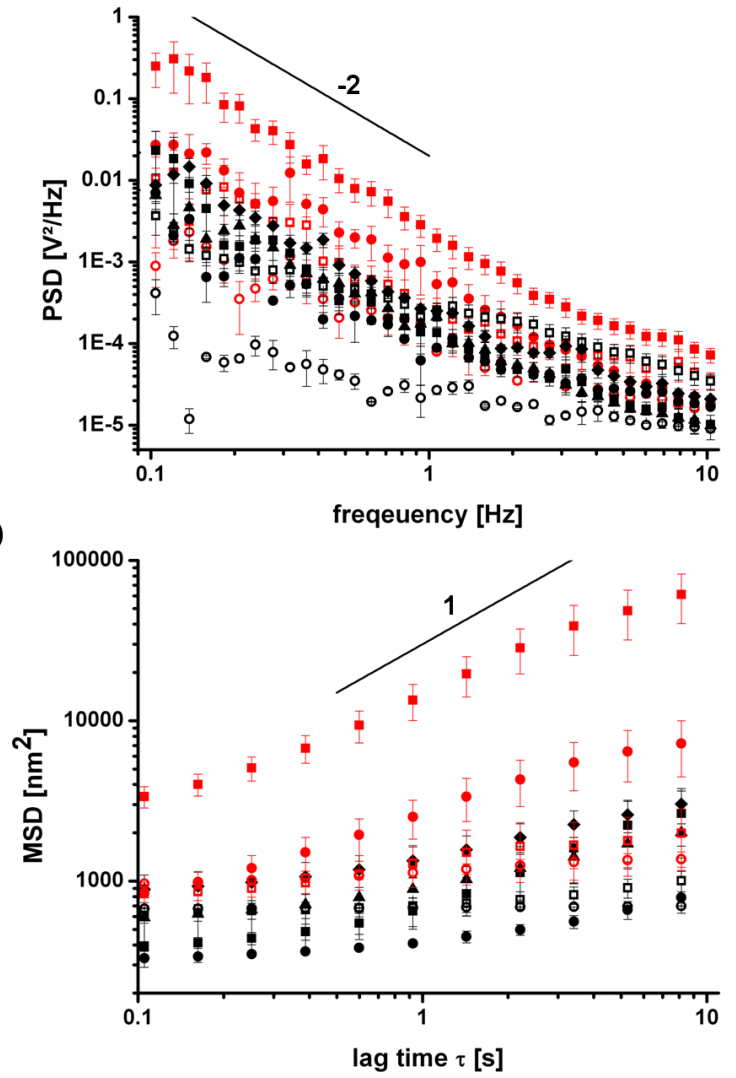

Figure 5.18: PSD and MSD analysis for DK4mer and Eg5

Open symbols correspond to motion perpendicular to the microtubule dumb-bell (shown only for filament angles of $0^{\circ}$ and $25^{\circ}$ ). A Power spectral density calculated for all suspended-microtubule assays (mean \pm SEM). B PSD plotted from 0.1 to $10 \mathrm{~Hz}$, the typical frequency range for motor activity. The slopes in the PSDs of the measurements at $0^{\circ}$ are $-1.81 \pm 0.05$ for DK4mer and $-1.31 \pm 0.04$ for Eg5. C Mean square displacement calculated for all suspended-microtubule assays (mean \pm SEM). D MSD plotted from 0.1 to $10 \mathrm{~s} \mathrm{lag}$, the typical time scale for motor activity. The slopes for the measurements at $0^{\circ}$ are $0.60 \pm 0.02$ for DK4mer and $0.31 \pm 0.05$ for Eg5. N: number of analyzed measurements (120 s each).

To further quantify these differences, we performed a frequency analysis of both motors under different filament angles and calculated the power spectral density of the recorded data (Figure 5.18 A and B). While the results for Eg5 (shown in black in Figure 5.18) are similar for the different angles, DK4mer (shown in red in Figure 5.18) shows a large discrepancy between both measurements. The same observation can be made when looking at the mean square displacement of the beads in the suspended-microtubule assay (Figure $5.18 \mathrm{C}$ and D). Almost no quantitative difference can be seen for the different Eg5 measurements, while the two results for DK4mer clearly differ. This supports our explanation that Eg5, as a wild type mitotic molecular motor, is well adapted to its task during mitosis, while an artificial tetrameric protein performs much worse under the same conditions.

Interestingly, Cahu and Surrey reported that the introduction of a comparable chimeric construct into Eg5 depleted $X$. laevis spindle extracts did not result in normal spindle morphogenesis but led to a new phenotype with an elongated collapsed microtubule bundle instead of a functional spindle [117]. They concluded that other factors than just the microtubule crosslinking activity and the plus-end 
directionality of Eg5 are required for a functional mitotic spindle. This strongly supports the findings we presented here.

The results presented here could not have been obtained with conventional experiments, like single-molecule fluorescence or single-bead motility assays. Our findings contradict previous results from relative-sliding assays and possibly indicate a new kind of regulatory mechanism that applies to mitotic Kinesin motor. We could demonstrate that the newly developed suspended-microtubule assay is a powerful tool for studying the properties of mitotic Kinesin motors in an environment closely resembling the conditions in the mitotic spindle. In addition, our experiments showed that the carefully tuned properties of a mitotic molecular motor cannot easily be copied. 



\section{Conclusions $\mid 6$}

Kinesin molecular motors are required for various forms of motility, ranging from intracellular transport to structural dynamics (as occurs in mitosis). Such diverse functions are possible because the structure and regulation of motors in each family can vary largely, despite strong similarities in their catalytic cores. The aim of this thesis was to investigate the regulatory mechanisms of Kinesin- 5 motor proteins. We were able to study the molecular mechanisms of examples of Kinesin-5 regulation by applying widely used techniques, like single-molecule fluorescence or single-bead motility assays, and by developing a new method, the suspended-microtubule assay.

\section{Neck-linker length dependence of processive Kinesin-5 motility}

One result, with potentially far reaching consequences, was discussed in Chapter 3. The processivity, i.e. the ability to take hundreds of successive steps without unbinding from the microtubule, of most members of the Kinesin family of motors raises the question of how the proteins manage to keep their two head domains out of phase. This is required for processive motion since at least one head has to be bound to the microtubule at any given time. The processivity of many Kinesin subfamilies suggests that an underlying mechanism exists which allows for head-head communication. This communication could be capable of regulating the efficiency of the motor, including the probability of backwards stepping and the direct coupling of the hydrolysis of exactly one ATP molecule to a single step.

It was argued that intramolecular tension, transmitted by the neck linker that connect the head domains to the stalk, could facilitate communication between both heads $[34,35,36]$. In this model, a shorter neck linker, which would lead to a tighter mechanical coupling, enhances communication and therefore results in a higher run length of the motor. A longer neck linker would weaken the mechanical coupling and ultimately diminish the ability of the motor to processively walk along a microtubule. Not only would this model explain how processive motion is achieved by molecular motors, it could also account for the different run lengths of the Kinesin subfamilies.

We have showed experimental evidence that contradicts this simple model. We studied the motile properties of twelve Kinesin- 5 head/Kinesin-1 stalk chimeras with different neck-linker length. Our findings indicate that the neck-linker length alone, and with it the strength of the mechanical coupling between the two head-domains, is insufficient to explain the motile properties of our constructs. We performed singlemolecule fluorescence as well as single-bead motility assays with all constructs and 
could not observe any influence of the neck-linker length on either velocity or force. We did, however, see an effect on the run length of the motors. We started from a neck linker that was too short to bridge the distance between two binding sites on the microtubule, effectively rendering the motor non-processive (NL-09). When we gradually extended the neck linker, the run length of the motor constructs increased to a peak value at a neck-linker length of 14 amino acids. This is in good agreement with the intramolecular strain model. Further extension of the neck linker, however, led to an additional increase of the run length until an global maximum was reached at 18 amino acids, which corresponds to the native neck linker length of Kinesin- 5. This contradicts the intramolecular strain model which predicts a reduced run length when elongating the neck linker from 14 to 18 amino acids. Elongation beyond 18 amino acids decreased the run length again. This was expected since an overly long neck linker will disrupt inter-head-communication at some point.

Our findings argue against the simple intramolecular strain model and instead point towards a different explanation. As supported by a recent study, head-head communication might be facilitated by the orientation of the neck linker [43]. This allows for a general explanation of processive motion of Kinesin motors while still offering the possibility that the mechanical properties of the neck linker, e.g. length or flexibility, can be optimized in different Kinesins for effective communication as well as other specific functions of the protein.

\section{Directionality of Cin8 molecules is mediated by the tail domains}

Another example for regulatory processes is the mediation of Kinesin-5 motility through the tail domains. It is believed that similar to the auto-inhibition of Kinesin1 [64, 65], the tail domains in Kinesin-5 can influence the motion of the protein. It was shown that the Kinesin-5 Eg5 from Xenopus laevis, when bound to a single microtubule, has two modes of motion: an ATP-dependent directional mode and a diffusive mode that does not require ATP hydrolysis [62]. Upon binding of a second microtubule, the diffusive mode of movement is suppressed and directed motion becomes dominant [62]. It therefore seems likely that the binding geometry of a Kinesin-5, i.e. whether it is bound to one or two microtubules, regulates its motile properties.

In this work (Chapter 3) and a previous study [68] we showed that the removal of the tail domains of Eg5 in a dimeric background results in a processive motor that is capable of directed motion without any diffusional component. The removal of the tail domains abolished the change of movement modes. This indicates that the tail domains are indeed required for a stable regulation of the motor's properties. The existence of microtubule binding sites in the tail domains of Eg5 [67] together with the recent observation that the tail and head domains are in close vicinity [66] further support this notion.

One striking instance of Kinesin-5 regulation, that was recently reported, is the switching of the direction of movement of Saccharomyces cerevisiae Kinesin-5 Cin8 [45, 46, 48]. Wild type Cin8, depending on the ionic strength of the surrounding medium, switches from a fast, processive, minus-end to a slow, erratic, plus-end directed motion. While it was initially argued that the collective behavior of multiple motors is required for the change of directionality [45], later studies showed that 
not only the change of ionic strength of the buffer, but also whether the motor is bound to one or two microtubules regulates its direction of movement [46, 47]. A similar ability to move processively toward both ends of a microtubule was observed for Kip1, the second Kinesin-5 homolog from S. cerevisiae [48]. It is not yet known if and how the microtubule binding geometry influences this motor. To answer the question whether Cin8 is, similar to Eg5, regulated by its tail domains, we studied two Cin8 constructs without tail domains in Chapter 4.

The Kinesin-5 head/Kinesin-1 stalk chimera Cin8Kin and the Cin8 $\Delta$ tail construct are both bidirectional motors, being able to move to the plus as well as minus ends of microtubules. Cin8Kin, however, moved in a quite erratic way while Cin8 $\Delta$ tail was highly directional. For Cin8 $\Delta$ tail in high ionic-strength buffer, individual runs were either plus- or minus-end directed while Cin8Kin changed its direction multiple times during a single run in all applied buffer conditions. We concluded that the tail domains are required for stable switching of directionality and are actually involved in the regulation of Cin8.

Furthermore, single-bead motility assays indicated that this regulation might be sensitive to forces. In a single-bead motility assay, Cin8Kin showed frequent changes of the direction of movement. The protein displaced optically trapped beads on average less than $100 \mathrm{~nm}$ before turning back, moving in one direction for a significantly shorter time compared to single-molecule fluorescence assays. The forces exerted by the trap in our study were rather weak $(\sim 0.5 \mathrm{pN})$. When applying a higher trap stiffness, resulting in higher forces, the motion appeared to be below the detection limit. The apparent, faster change between forward and backward motion upon reaching a certain force value might point toward a mechanism that is capable of tuning the motility of the motor according to the surrounding conditions.

Thus, we could show that the behavior of Cin8 motors can be explained by the possible regulation of molecular motors by interactions with their tail domains. Taking into account the differences between the Cin8Kin chimera and the Cin8 $\Delta$ tail, there are most likely other elements involved as well. The findings presented here demonstrate that the tail domains are certainly required for a well defined regulation of Cin8. The recent report that the second Kinesin-5 homolog in yeast, Kip1, is also capable of bidirectional motion and more importantly that its motility is comparable to the Cin8Kin chimera [48] further supports the existence of a conserved regulatory process in, at least, yeast Kinesin-5 motors.

In light of the predominant role of Cin8 in mitosis, a force sensitive regulation seems plausible. The mitotic spindle is complicated machinery that has to be delicately fine-tuned to enable cell division. It is imperative that a balance of forces is achieved during the various stages of cell division has to be achieved. A motor, which motile properties adapt to outside forces, might therefore be highly favorable.

\section{In vitro studies of mitotic Kinesin motors in their native environment}

So far all of the studies concerning Kinesin- 5 motors were done in artificial environments. The previous reports about Eg5's force-generating capabilities, for example, have been done with single-bead motility assays [10]. In these experiments, the motors are attached to micrometer sized spheres, which is certainly a non-native conformation. Likewise, in single-molecule fluorescence assays, mitotic motors are 
studied on single microtubules $[46,62]$, or in the case of the relative-sliding assay, while crosslinking unloaded microtubules $[9,47]$. While the latter is close to the actual situation in the mitotic spindle, the lack of forces might still influence the motile properties. The already mentioned possibility of a force sensitive regulation in Cin8 motors cannot be observed by these means.

To overcome these limitations, we introduced a new kind of optical trapping assay in Chapter 5. In the suspended-microtubule assay, biotinylated microtubules are bound to two neutravidin coated beads. With an optical trap, this microtubule dumb-bell is antiparallelly aligned with a surface-immobilized axoneme to resemble the geometry of the mitotic spindle. This enables us to study the motile properties and regulatory mechanisms of mitotic motors in a loaded environment similar to the mitotic spindle. In Chapter 5, we presented the results from this new assay for two different motors: the fast, chimeric microtubule-sliding motor DK4mer and the slow, mitotic Kinesin-5 Eg5 from Xenopus laevis. For the first time we could show the actual behavior of mitotic Kinesin motors in a loaded environment. This presented us with some previously unobservable results.

While considerably faster than Eg5, the artificial chimera DK4mer proved to be less adapted to microtubule crosslinking. Upon introduction of an angle of $25^{\circ}$ between the two filaments, the velocity as well as the force was considerably reduced by a factor of more than two. Eg5 on the other hand, being a native mitotic motor, was a lot less susceptible to filament angles. Forces as well as velocities stayed constant for angles up to $25^{\circ}$. In a recent study, Cahu et al. injected a construct similar to DK4mer into Eg5 depleted X. laevis spindle extracts [117]. The spindles first evolved from elongated, distorted spindles to collapsed spindles with chromatin in the center and finally to completely collapsed microtubule bundles with the chromatin mass at one end of the bundle [117]. They concluded that the microtubule-crosslinking activity and plus-end directionality of Kinesin-5 alone might not be sufficient for the function of Eg5 and maybe other factors are involved. Our findings suggest that the mechanical properties of the protein, in particular the dependence of the motility on filament angle, influence its role in mitosis. One possible explanation could be the design of the neck linker. As indicated in Chapter 3, not only the length but also the orientation - and possibly the sequence of the neck linker - might influence the motile properties of a protein. The neck linker in DK4mer is of artificial design. It consists of the sequence of the DmKHC neck linker extended by the last four amino acids of the Eg5 neck linker. This could very well influence its ability to crosslink poorly aligned microtubules.

Following up on previous studies of Eg5 [9, 62], our suspended-microtubule assay provided further evidence that Eg5 is regulated by microtubule binding geometry. In contrast to single-bead motility assays, we here measured forces that are in excess of $3 \mathrm{pN}$, twice the previously reported value [10]. Together with the enhancement of active, directional motion upon binding of a second microtubule, this completes the notion that wild type Eg5 is highly (down)regulated in its native tetrameric form when not bound to two microtubules. This might be useful in the mitotic spindle, where various processes have to be carefully balanced and adjusted in the different stages of cell division.

In the mitotic spindle, as well as in the suspended-microtubule assay, the filaments are aligned antiparallel with respect to each other. Given the tetrameric structure of mitotic Kinesins, it is to be expected that the protein walks on both filaments 
it crosslinks. The relative velocity with which both microtubules move away from each other is therefore two times the motor's velocity. For DK4mer and Eg5, this has been confirmed by relative-sliding assays [9, 69].

Surprisingly, the results from the suspended-microtubule assay contradict these findings. DK4mer as well as Eg5, consistently showed velocities corresponding to the velocity of the motors on a single microtubule. This suggests that the relative-sliding assays - which, in contrast to the mitotic spindle, are load free - might not provide reliable data when analyzing relative sliding velocities. Based on these findings, we proposed a previously unobserved regulatory mechanism: The processivity of a motor depends, among other things, on the probability of the unbinding of both heads at the same time (see Section 1.1). Mechanisms, like the regulation of the chemo-mechanical cycle by the neck linker discussed in Chapter 3, ensure that the motor stays bound for longer periods of time.

A tetrameric motor in the mitotic spindle is bound with both head groups to different microtubules. If the motor processively walks along both microtubules, the probability of losing connection with at least one of the microtubule is doubled. For the maintenance of the mitotic spindle, this could be a disadvantage. It therefore seems plausible that the motor, when under load, does not walk on both microtubules at the same time but instead walks either on one or alternating on both microtubules. So far, our data does not allow us to distinguish between both scenarios, but it certainly confirmed that the studied motors are not processively moving on both microtubules at the same time. Further studies might shed some light on the exact details.

With the work presented here, we could establish the suspended-microtubule assay as a promising new technique which will hopefully be used to gain deeper knowledge of mitotic motors in their native environment. For example the question presented in Chapter 4 - how the directionality of Cin8 is regulated by the tail domains - might finally be answered by this assay.

In recent years, it has slowly become more obvious that Kinesin motor proteins are by no means the simple, ATP-hydrolyzing micro-engines they were believed to be. Instead, they seem to be highly adapted, specifically tuned machines. The investigation of this "tuning", i.e. regulation, of motor proteins has come to increasing attention. However, all the appealing models which try to predict the general underlying principles have failed and more complex explanations were needed. The work presented here offers further insight into the complicated mechanisms of Kinesin5 regulation, further supporting the notion that the processes involving molecular motors are more complicated than previously thought. While this work does not claim to be exhaustive, the results as well as the new method described here may aid in tackling some of the questions still unanswered. It certainly seems unlikely that there will be any lack of unanswered questions in the near future. To quote Steven M. Block, one of the pioneers of motor protein studies with optical traps, [36]:

"A great deal more remains to be discovered about motor proteins. Nature is vastly more subtle, and generally smarter, than we tend to give her credit for being." 

Appendices 



\section{Biochemical protocols}

If not indicated otherwise, all chemicals and materials were obtained from SigmaAldrich Chemie Gmbh (Munich, Germany).

\section{Coating of beads}

\section{His-antibody coating}

1. dilute $10 \mu \mathrm{l}$ bead solution (silica, $1 \mu \mathrm{m}$, plain, 1:10 in $\mathrm{H}_{2} \mathrm{O}$; Kisker Biotech GmbH \& Co. KG, Steinfurt, Germany) in $80 \mu \mathrm{l}$ BRB80

2. add $10 \mu$ protein-G $(50 \mathrm{mg} / \mathrm{ml})$

3. incubate $1 \mathrm{~h}$ at $4^{\circ} \mathrm{C}$ while shaking

4. centrifuge for $5 \mathrm{~min}$ at $7800 \mathrm{~g}$, RT

5. remove $100 \mu \mathrm{l}$ supernatant

6. resolve pellet in $90 \mu \mathrm{l} \mathrm{BRB} 80$

7. add $10 \mu \mathrm{l}$ Penta-His-antibody $(1 \mathrm{mg} / \mathrm{ml})$

8. incubate $1 \mathrm{~h}$ at $4^{\circ} \mathrm{C}$ while shaking

9. centrifuge for $5 \mathrm{~min}$ at $7800 \mathrm{~g}$, RT

10. remove $100 \mu \mathrm{l}$ supernatant

11. resolve pellet in $198 \mu \mathrm{l} \mathrm{BRB} 80$

12. add $2 \mu \mathrm{l}$ ATP $(100 \mathrm{mM})$

13. store at $4^{\circ} \mathrm{C}$ until use 


\section{Neutravidin coating}

Biotinylation of beads (used biotin: 6-((6-((biotinoyl)amino)hexanoyl)amino)hexanoic acid, succinimidyl ester):

1. dilute $50 \mu \mathrm{l}$ bead stock-solution (silica, $1.5 \mu \mathrm{m}$, carboxilated; Kisker Biotech GmbH \& Co. KG, Steinfurt, Germany) in $950 \mu$ phosphate buffer

2. centrifuge for $10 \mathrm{~min}$ at $7800 \mathrm{~g}, \mathrm{RT}$

3. resolve pellet in $800 \mu$ l phosphate buffer

4. add $100 \mu \mathrm{EDC}(75 \mathrm{mg} / \mathrm{ml})$

5. add $100 \mu$ Biotin-XX, SE (1 mg/ml)

6. incubate for $1 \mathrm{~h}$ at RT while shaking

7. centrifuge for $10 \mathrm{~min}$ at $7800 \mathrm{~g}$, RT

8. resolve pellet in $1000 \mu \mathrm{l}$ phosphate buffer

9. repeat 7 . and 8 .

10. store at $4^{\circ} \mathrm{C}$ until use

Applying neutravidin

1. dilute $25 \mu \mathrm{l}$ of biotinylated beads in $55 \mu \mathrm{l}$ phosphate buffer

2. add $10 \mu$ l glycine $(1 \mathrm{M})$

3. add $10 \mu \mathrm{l}$ neutravidin $(5 \mathrm{mg} / \mathrm{ml})$

4. incubate for $1 \mathrm{~h}$ at $\mathrm{RT}$ while shaking

5. centrifuge for $10 \mathrm{~min}$ at $7800 \mathrm{~g}$, RT

6. resolve pellet in $100 \mu$ l phosphate buffer

7. repeat 5 . and 6 .

8. store at $4^{\circ} \mathrm{C}$ until use 


\section{Preparation of microtubules}

\section{TMR-labeled microtubules}

1. to $19 \mu \mathrm{l}$ BRB80

2. add $2 \mu$ tubulin $(15 \mathrm{mg} / \mathrm{ml}$ in P12)

3. add $2 \mu \mathrm{l}$ TMR-tubulin $(15 \mathrm{mg} / \mathrm{ml}$ in $\mathrm{P} 12)$

4. add $1 \mu \mathrm{l}$ GTP $\left(25 \mathrm{mM}\right.$ in $\left.\mathrm{H}_{2} \mathrm{O}\right)$

5. add $1 \mu \mathrm{l} \mathrm{MgCl}_{2}(200 \mathrm{mM}$ in $\mathrm{P} 12)$

6. incubate $15 \mathrm{~min}$ at $37^{\circ} \mathrm{C}$

7. add $100 \mu \mathrm{l}$ BRB-taxol solution (1 $\mu \mathrm{M}$ taxol in BRB80)

8. store at RT until use

\section{Biotin-labeled microtubules}

1. to $19 \mu \mathrm{l}$ BRB80

2. add $1.5 \mu$ l tubulin $(15 \mathrm{mg} / \mathrm{ml}$ in $\mathrm{P} 12)$

3. add $2 \mu \mathrm{l}$ TMR-tubulin (15 mg/ml in P12)

4. add $0.5 \mu$ l biotinylated tubulin $(15 \mathrm{mg} / \mathrm{ml}$ in $\mathrm{P} 12)$

5. add $1 \mu \mathrm{l}$ GTP $\left(25 \mathrm{mM}\right.$ in $\left.\mathrm{H}_{2} \mathrm{O}\right)$

6. add $1 \mu \mathrm{l} \mathrm{MgCl}_{2}(200 \mathrm{mM}$ in P12)

7. incubate $15 \mathrm{~min}$ at $37^{\circ} \mathrm{C}$

8. add $100 \mu \mathrm{l}$ BRB-taxol solution (1 $\mu \mathrm{M}$ taxol in BRB80)

9. store at RT until use

\section{Polarity marked microtubules}

For fluorescent labeling of the minus end of microtubules, a two step process is used. Seeds:

1. to $17 \mu \mathrm{l}$ BRB80

2. add $5 \mu \mathrm{l}$ TMR-tubulin $(15 \mathrm{mg} / \mathrm{ml}$ in P12)

3. add $1 \mu \mathrm{l}$ NEM-tubulin $(15 \mathrm{mg} / \mathrm{ml}$ in P12) 
4. add $1 \mu$ l GMP-CPP $\left(25 \mathrm{mM}\right.$ in $\left.\mathrm{H}_{2} \mathrm{O}\right)$

5. add $1 \mu \mathrm{l} \mathrm{MgCl}_{2}(200 \mathrm{mM}$ in $\mathrm{P} 12)$

6. incubate $10 \mathrm{~min}$ at $37^{\circ} \mathrm{C}$

7. store at RT until use

Elongation of seeds:

1. to $19.5 \mu \mathrm{BRB} 80$

2. add $1.5 \mu$ tubulin $(15 \mathrm{mg} / \mathrm{ml}$ in $\mathrm{P} 12)$

3. add $0.5 \mu \mathrm{l}$ TMR-tubulin $(15 \mathrm{mg} / \mathrm{ml}$ in $\mathrm{P} 12)$

4. add $0.5 \mu \mathrm{l}$ NEM-tubulin $(15 \mathrm{mg} / \mathrm{ml}$ in P12)

5. add $1 \mu \mathrm{l}$ GTP $\left(25 \mathrm{mM}\right.$ in $\left.\mathrm{H}_{2} \mathrm{O}\right)$

6. add $1 \mu \mathrm{l} \mathrm{MgCl}_{2}(200 \mathrm{mM}$ in $\mathrm{P} 12)$

7. add $1 \mu \mathrm{l}$ of $1: 10$ diluted seeds

8. incubate $15 \mathrm{~min}$ at $37^{\circ} \mathrm{C}$

9. add $100 \mu \mathrm{l}$ BRB-taxol solution $(10 \mu \mathrm{M}$ taxol in BRB80)

10. store at RT until use

\section{Cleaning of microtubules}

1. put $99 \mu \mathrm{l}$ of 50:50 BRB80:glycerol solution into reaction tube

2. add $1 \mu$ of taxol $(1 \mathrm{mM})$

3. carefully add $50 \mu$ l of microtubule solution on top without mixing

4. centrifuge for $10 \mathrm{~min}, 7800 \mathrm{~g}$, RT

5. carefully remove $50 \mu \mathrm{l}$ from the bottom and put into new tube

6. store at RT until use 


\section{Preparation of cover slips}

\section{DETA coating}

1. clean cover slips for $10 \mathrm{~min}$, using a plasma cleaner

2. immerse cover slips in a solution containing $~ 0.1 \%$ DETA

3. sonificate for $5 \mathrm{~min}$

4. immerse cover slips in water

5. sonificate for $5 \mathrm{~min}$

6. repeat 4 . and 5 .

7. dry cover slips for $\sim 1$ h at $\sim 75^{\circ} \mathrm{C}$

\section{Microstructuring}

For the fabrication of microstructures on glass cover slips, SU8-2001 photoresist (Michrochem, Newton, MA, USA; kindly provided by Sarah Köster) was used. To ensure, that the photoresist properly adheres to the glass, a highly clean environment was necessary. Therefore, all processes were performed in a clean room, fulfilling the ISO 5 standard.

1. clean cover slips for 10 min, using a plasma cleaner

2. add $\sim 800 \mu \mathrm{l}$ SU8 on the cover slip

3. spincoat cover slip: $10 \mathrm{~s}$ at $500 \mathrm{rpm}(100 \mathrm{rpm} / \mathrm{s}$ ramp), followed by $30 \mathrm{~s}$ at $1000 \mathrm{rpm}(300 \mathrm{rpm} / \mathrm{s} \mathrm{ramp})$

4. soft-bake cover slip on heating plate for $60 \mathrm{~s}$ at $95^{\circ} \mathrm{C}$

5. through photomask (4 inch sodalime glass, evaporated with chromium; ML\&C, Jena-Maua, Germany), expose cover slip to UV-light for 4 s, 22 $\mathrm{mW} / \mathrm{cm}^{2}$ intensity

6. hard-bake cover slip on heating plate for $120 \mathrm{~s}$ at $95^{\circ} \mathrm{C}$

7. develop cover slip by immersing into SU8 developer (PGMEA developer, Michrochem, Newton, MA, USA) for $60 \mathrm{~s}$ 


\section{A.4 Isolation and purification of axonemes}

The described procedures for culturing and harvesting Chlamydomonas reinhardtii green algae, the isolation of their flagella and the purification of axonemes from these flagella was, if not indicated otherwise, taken from the work of G.B. Witman [116]. In the present work the Chlamydomonas reinhardtii strain CC-2228 (Chlamydomonas Resource Center of the University of Minnesota, St. Paul, MN, USA) was used.

\section{TAP medium}

from Gorman, D.S., and R.P. Levine [118]. Procedure taken from the website of the Chlamydomonas Resource Center of the University of Minnesota (St. Paul, MN, USA).

1. TAP salts

- $\mathrm{NH}_{4} \mathrm{Cl} 15.0 \mathrm{~g}$

- $\mathrm{MgSO}_{4} \cdot 7 \mathrm{H}_{2} \mathrm{O} 4.0 \mathrm{~g}$

- $\mathrm{CaCl}_{2} \cdot 2 \mathrm{H}_{2} \mathrm{O} 2.0 \mathrm{~g}$

- water to 1 liter

2. phosphate solution

- $\mathrm{K}_{2} \mathrm{HPO}_{4} 28.8 \mathrm{~g}$

- $\mathrm{KH}_{2} \mathrm{PO}_{4} 14.4 \mathrm{~g}$

- water to $100 \mathrm{ml}$

3. Hutner's trace elements

To make the final medium, mix the following:

- $2.42 \mathrm{~g}$ Tris

- $25 \mathrm{ml}$ solution 1. (TAP salts)

- $0.375 \mathrm{ml}$ solution 2. (phosphate solution)

- $1.0 \mathrm{ml}$ solution 3. (trace elements)

- $1.0 \mathrm{ml}$ glacial acetic acid

- water to 1 liter

- autoclave 


\section{Hutner's trace elements}

from Hutner et al. [119]. Procedure taken from the website of the Chlamydomonas Resource Center of the University of Minnesota (St. Paul, MN, USA).

For a detailed analysis of how well this trace elements solution meets the nutritional requirements of C. reinhardtii, see Merchant et al. [120].

For 1 liter final mix, dissolve each compound in the volume of water indicated. The EDTA should be dissolved in boiling water, and the $\mathrm{FeSO}_{4}$ should be prepared last to avoid oxidation.

- EDTA disodium salt $50 \mathrm{~g}(250 \mathrm{ml})$

- $\mathrm{ZnSO}_{4} \cdot 7 \mathrm{H}_{2} \mathrm{O} 22 \mathrm{~g}(100 \mathrm{ml})$

- $\mathrm{H}_{3} \mathrm{BO}_{3} 11.4 \mathrm{~g}(200 \mathrm{ml})$

- $\mathrm{MnCl}_{2} \cdot 4 \mathrm{H}_{2} \mathrm{O} 5.06 \mathrm{~g}(50 \mathrm{ml})$

- $\mathrm{CoCl}_{2} \cdot 6 \mathrm{H}_{2} \mathrm{O} 1.61 \mathrm{~g}(50 \mathrm{ml})$

- $\mathrm{CuSO}_{4} \cdot 5 \mathrm{H}_{2} \mathrm{O} 1.57 \mathrm{~g}(50 \mathrm{ml})$

- $\left(\mathrm{NH}_{4}\right)_{6} \mathrm{Mo}_{7} \mathrm{O}_{24} \cdot 4 \mathrm{H}_{2} \mathrm{O} 1.10 \mathrm{~g}(50 \mathrm{ml})$

- $\mathrm{FeSO}_{4} \cdot 7 \mathrm{H}_{2} \mathrm{O} 4.99 \mathrm{~g}(50 \mathrm{ml})$

Mix all solutions except EDTA. Bring to boil, then add EDTA solution. The mixture should turn green. When everything is dissolved, cool to $70^{\circ} \mathrm{C}$. Keeping temperature at $70^{\circ} \mathrm{C}$, add $85 \mathrm{ml}$ hot $20 \% \mathrm{KOH}$ solution (20 g / $100 \mathrm{ml}$ final volume). Do NOT use $\mathrm{NaOH}$ to adjust the $\mathrm{pH}$.

Bring the final solution to 1 liter total volume. It should be clear green initially. Stopper the flask with a cotton plug and let it stand for 1-2 weeks, shaking it once a day. The solution should eventually turn purple and leave a rust-brown precipitate, which can be removed by filtering through two layers of Whatman\#1 filter paper, repeating the filtration if necessary until the solution is clear. Store refrigerated or frozen convenient aliquots. Some people shorten the time for formation of the precipiate by bubbling the solution with filtered air.

If no precipitate forms, the solution is still usable. However, you might want to check the $\mathrm{pH}$ in this case and adjust it to around 7.0 using either $\mathrm{KOH}$ or $\mathrm{HCl}$ as needed. To prepare sulfur-free trace elements for hydrogen generation, the sulfate salts can be replaced with equimolar chloride salts $\left(\mathrm{ZnCl}_{2} 10.0 \mathrm{~g} ; \mathrm{CuCl}_{2} \cdot 2 \mathrm{H}_{2} \mathrm{O}\right.$ $\left.1.00 \mathrm{~g} ; \mathrm{FeCl}_{2} \cdot 4 \mathrm{H} 2 \mathrm{O}, 3.60 \mathrm{~g}\right)$. 


\section{Chlamydomonas flagella isolation - solutions}

HMDS

- 10 mM HEPES

- $5 \mathrm{mM} \mathrm{MgSO}_{4}$

- $1 \mathrm{mM}$ DTT

- $4 \%$ sucrose

- $\mathrm{pH} 7.4$

HMDS-EGTA

- 28 ml HMDS

- $140 \mu \mathrm{l} 0.1 \mathrm{M}$ EGTA

HMDS-sucrose

- 10 mM HEPES

- $5 \mathrm{mM} \mathrm{MgSO}_{4}$

- $1 \mathrm{mM}$ DTT

- $25 \%$ sucrose

- $\mathrm{pH} 7.4$

\section{HMDEKP}

- 30 mM HEPES

- $5 \mathrm{mM} \mathrm{MgSO}_{4}$

- $1 \mathrm{mM}$ DTT

- $0.5 \mathrm{mM} \mathrm{Na}_{2}$ EDTA

- $25 \mathrm{mM} \mathrm{KCl}$

- $0.5 \%$ polyethylene glycol, MW 20,000

- $\mathrm{pH} 7.4$

HMDEKP-Igepal

- 30 mM HEPES 
- $5 \mathrm{mM} \mathrm{MgSO}_{4}$

- $1 \mathrm{mM}$ DTT

- $0.5 \mathrm{mM} \mathrm{Na} \mathrm{maDT}_{2}$

- $25 \mathrm{mM} \mathrm{KCl}$

- $0.5 \%$ polyethylene glycol, MW 20,000

- $1 \%$ Igepal

- $\mathrm{pH} 7.4$

\section{Chlamydomonas flagella isolation}

(for 21 cultures)

Harvesting cells

1. distribute cells in $500 \mathrm{ml}$ centrifuge tubes

2. centrifuge $10 \mathrm{~min}$ at $1100 \mathrm{~g}, \mathrm{RT}$

3. remove supernatant

4. resuspend cells in $25 \mathrm{ml}$ of $10 \mathrm{mM}$ HEPES buffer

5. wash once by repeating steps 2 to 4

Isolation of flagella

1. centrifuge $10 \mathrm{~min}$ at $1100 \mathrm{~g}, \mathrm{RT}$

2. remove supernatant

3. resuspend cells in ice cold HMDS solution (total volume: $2.5 \mathrm{x}$ packed cell volume, at least $10 \mathrm{ml}$ )

4. place $10 \mathrm{ml}$ aliquots of the cell suspension into $50 \mathrm{ml}$ centrifuge tubes

5. deflagellate cells by adding $2 \mathrm{ml}$ of $25 \mathrm{mM}$ dibucaine to each tube and vigorously pipette the suspension

6. immediately add $28 \mathrm{ml}$ of ice cold HMDS-EGTA to each tube

7. centrifuge $5 \mathrm{~min}$ at $1800 \mathrm{~g}, 4^{\circ} \mathrm{C}$

8. collect supernatant an distribute into clean $50 \mathrm{ml}$ centrifuge tubes ( $\sim 35$ $\mathrm{ml}$ per tube)

9. underlay with $10 \mathrm{ml}$ ice cold HMDS-sucrose 
10. centrifuge $10 \mathrm{~min}$ at $2400 \mathrm{~g}, 4^{\circ} \mathrm{C}$

11. collect upper layer $(\sim 25 \mathrm{ml}$ per tube)[if cell bodies remain in solution, repeat steps 7 to 9$]$

12. distribute suspension into $25 \mathrm{ml}$ ultra-centrifuge tubes

13. centrifuge $20 \mathrm{~min}$ at $31,000 \mathrm{~g}, 4^{\circ} \mathrm{C}$

14. discard supernatant

Demembranation and isolation of axonemes

DO NOT USE GLASS EQUIPMENT!!

1. resuspend isolated flagella in ice cold HMDEKP-Igepal (total volume: $1.5 \mathrm{x}$ packed cell volume $[\sim 10 \mathrm{ml}]$ )

2. distribute suspension into $10 \mathrm{ml}$ ultra-centrifuge tubes

3. centrifuge $20 \mathrm{~min}$ at $31,000 \mathrm{~g}, 4^{\circ} \mathrm{C}$

4. discard supernatant

5. repeat steps 1 to 3

6. resuspend the axonemes in ice cold HMDEKP and wash by centrifugation as above

7. distribute the axonemes into $50 \mu \mathrm{l}$ aliquots in $0.5 \mathrm{ml}$ tubes and freeze by immersion in liquid nitrogen

8. store frozen axonemes at $-80^{\circ} \mathrm{C}$ until use 


\section{List of references}

[1] E. Schrödinger, What is Life? Cambridge University Press, 1944.

[2] D. Mizuno, C. Tardin, C. F. Schmidt, and F. C. Mackintosh, "Nonequilibrium mechanics of active cytoskeletal networks.," Science, vol. 315, pp. 370-373, Jan 2007.

[3] H. Nyquist, "Thermal agitation of electric charge in conductors," Physical Review, vol. 32, pp. 110-113, 1928.

[4] R. D. Vale, T. S. Reese, and M. P. Sheetz, "Identification of a novel forcegenerating protein, kinesin, involved in microtubule-based motility.," Cell, vol. 42, pp. 39-50, Aug 1985.

[5] C. J. Lawrence, R. K. Dawe, K. R. Christie, D. W. Cleveland, S. C. Dawson, S. A. Endow, L. S. B. Goldstein, H. V. Goodson, N. Hirokawa, J. Howard, R. L. Malmberg, J. R. McIntosh, H. Miki, T. J. Mitchison, Y. Okada, A. S. N. Reddy, W. M. Saxton, M. Schliwa, J. M. Scholey, R. D. Vale, C. E. Walczak, and L. Wordeman, "A standardized kinesin nomenclature.," J. Cell Biol., vol. 167, pp. 19-22, Oct 2004.

[6] K. Visscher, M. J. Schnitzer, and S. M. Block, "Single kinesin molecules studied with a molecular force clamp.," Nature, vol. 400, pp. 184-189, Jul 1999.

[7] K. Svoboda, C. F. Schmidt, B. J. Schnapp, and S. M. Block, "Direct observation of kinesin stepping by optical trapping interferometry.," Nature, vol. 365, pp. 721-727, Oct 1993.

[8] A. S. Kashina, G. C. Rogers, and J. M. Scholey, "The bimc family of kinesins: essential bipolar mitotic motors driving centrosome separation.," Biochim. Biophys. Acta, vol. 1357, pp. 257-271, Jul 1997.

[9] L. C. Kapitein, E. J. G. Peterman, B. H. Kwok, J. H. Kim, T. M. Kapoor, and C. F. Schmidt, "The bipolar mitotic kinesin eg5 moves on both microtubules that it crosslinks.," Nature, vol. 435, pp. 114-118, May 2005.

[10] M. J. Korneev, S. Lakämper, and C. F. Schmidt, "Load-dependent release limits the processive stepping of the tetrameric eg5 motor.," Eur. Biophys. J., vol. 36, pp. 675-681, Jul 2007. 
[11] G. Steinberg and M. Schliwa, "Characterization of the biophysical and motility properties of kinesin from the fungus neurospora crassa.," J. Biol. Chem., vol. 271, pp. 7516-7521, Mar 1996.

[12] S. Lakämper, A. Kallipolitou, G. Woehlke, M. Schliwa, and E. Meyhöfer, "Single fungal kinesin motor molecules move processively along microtubules.," Biophys. J., vol. 84, pp. 1833-1843, Mar 2003.

[13] W. Hua, J. Chung, and J. Gelles, "Distinguishing inchworm and hand-overhand processive kinesin movement by neck rotation measurements.," Science, vol. 295, pp. 844-848, Feb 2002.

[14] C. L. Asbury, A. N. Fehr, and S. M. Block, "Kinesin moves by an asymmetric hand-over-hand mechanism.," Science, vol. 302, pp. 2130-2134, Dec 2003.

[15] K. Kaseda, H. Higuchi, and K. Hirose, "Alternate fast and slow stepping of a heterodimeric kinesin molecule.," Nat. Cell Biol., vol. 5, pp. 1079-1082, Dec 2003 .

[16] B. Gutiérrez-Medina, A. N. Fehr, and S. M. Block, "Direct measurements of kinesin torsional properties reveal flexible domains and occasional stalk reversals during stepping.," Proc. Natl. Acad. Sci. U. S. A., vol. 106, pp. 17007-17012, Oct 2009 .

[17] A. Yildiz, M. Tomishige, R. D. Vale, and P. R. Selvin, "Kinesin walks handover-hand.," Science, vol. 303, pp. 676-678, Jan 2004.

[18] S. C. Kuo, J. Gelles, E. Steuer, and M. P. Sheetz, "A model for kinesin movement from nanometer-level movements of kinesin and cytoplasmic dynein and force measurements.," J Cell Sci Suppl, vol. 14, pp. 135-138, 1991.

[19] S. Ray, E. Meyhöfer, R. A. Milligan, and J. Howard, "Kinesin follows the microtubule's protofilament axis.," J. Cell Biol., vol. 121, pp. 1083-1093, Jun 1993.

[20] S. M. Block and K. Svoboda, "Analysis of high resolution recordings of motor movement.," Biophys. J., vol. 68, pp. 230S-239S; discussion 239S-241S, Apr 1995.

[21] R. A. Cross, "On the hand-over-hand footsteps of kinesin heads.," J. Muscle Res. Cell. Motil., vol. 16, pp. 91-94, Apr 1995.

[22] A. N. Fehr, C. L. Asbury, and S. M. Block, "Kinesin steps do not alternate in size.," Biophys. J., vol. 94, pp. L20-L22, Feb 2008.

[23] A. N. Fehr, B. Gutiérrez-Medina, C. L. Asbury, and S. M. Block, "On the origin of kinesin limping.," Biophys. J., vol. 97, pp. 1663-1670, Sep 2009.

[24] I. A. T. Schaap, C. Carrasco, P. J. de Pablo, and C. F. Schmidt, "Kinesin walks the line: single motors observed by atomic force microscopy.," Biophys. J., vol. 100, pp. 2450-2456, May 2011. 
[25] H. B. McDonald, R. J. Stewart, and L. S. Goldstein, "The kinesin-like ncd protein of drosophila is a minus end-directed microtubule motor.," Cell, vol. 63, pp. 1159-1165, Dec 1990.

[26] E. P. Sablin, R. B. Case, S. C. Dai, C. L. Hart, A. Ruby, R. D. Vale, and R. J. Fletterick, "Direction determination in the minus-end-directed kinesin motor ncd.," Nature, vol. 395, pp. 813-816, Oct 1998.

[27] M. J. deCastro, R. M. Fondecave, L. A. Clarke, C. F. Schmidt, and R. J. Stewart, "Working strokes by single molecules of the kinesin-related microtubule motor ncd," Nat. Cell Biol., vol. 2, pp. 724-729, Oct 2000.

[28] J. Howard, A. J. Hudspeth, and R. D. Vale, "Movement of microtubules by single kinesin molecules.," Nature, vol. 342, pp. 154-158, Nov 1989.

[29] R. D. Vale, T. Funatsu, D. W. Pierce, L. Romberg, Y. Harada, and T. Yanagida, "Direct observation of single kinesin molecules moving along microtubules.," Nature, vol. 380, pp. 451-453, Apr 1996.

[30] J. M. Scholey, I. Brust-Mascher, and A. Mogilner, "Cell division.," Nature, vol. 422, pp. 746-752, Apr 2003.

[31] N. Hirokawa, Y. Noda, Y. Tanaka, and S. Niwa, "Kinesin superfamily motor proteins and intracellular transport.," Nat. Rev. Mol. Cell Biol., vol. 10, pp. 682-696, Oct 2009 .

[32] S. S. Rosenfeld, M. van Duffelen, W. M. Behnke-Parks, C. Beadle, J. Corrreia, and J. Xing, "The atpase cycle of the mitotic motor cenp-e.," J. Biol. Chem., vol. 284, pp. 32858-32868, Nov 2009.

[33] S. Shastry and W. O. Hancock, "Interhead tension determines processivity across diverse n-terminal kinesins.," Proc. Natl. Acad. Sci. U. S. A., vol. 108, pp. 16253-16258, Sep 2011.

[34] R. D. Vale and R. A. Milligan, "The way things move: looking under the hood of molecular motor proteins.," Science, vol. 288, pp. 88-95, Apr 2000.

[35] S. S. Rosenfeld, P. M. Fordyce, G. M. Jefferson, P. H. King, and S. M. Block, "Stepping and stretching. how kinesin uses internal strain to walk processively.," J. Biol. Chem., vol. 278, pp. 18550-18556, May 2003.

[36] S. M. Block, "Kinesin motor mechanics: binding, stepping, tracking, gating, and limping.," Biophys. J., vol. 92, pp. 2986-2995, May 2007.

[37] F. Kozielski, S. Sack, A. Marx, M. Thormählen, E. Schönbrunn, V. Biou, A. Thompson, E. M. Mandelkow, and E. Mandelkow, "The crystal structure of dimeric kinesin and implications for microtubule-dependent motility.," Cell, vol. 91, pp. 985-994, Dec 1997.

[38] S. Rice, A. W. Lin, D. Safer, C. L. Hart, N. Naber, B. O. Carragher, S. M. Cain, E. Pechatnikova, E. M. Wilson-Kubalek, M. Whittaker, E. Pate, R. Cooke, E. W. Taylor, R. A. Milligan, and R. D. Vale, "A structural change in the 
kinesin motor protein that drives motility.," Nature, vol. 402, pp. 778-784, Dec 1999.

[39] D. D. Hackney, "The tethered motor domain of a kinesin-microtubule complex catalyzes reversible synthesis of bound atp.," Proc. Natl. Acad. Sci. U. S. A., vol. 102, pp. 18338-18343, Dec 2005.

[40] M. L. Kutys, J. Fricks, and W. O. Hancock, "Monte carlo analysis of neck linker extension in kinesin molecular motors.," PLoS Comput. Biol., vol. 6, no. 11, p. e1000980, 2010.

[41] I. M.-T. C. Crevel, M. C. Alonso, and R. A. Cross, "Monastrol stabilises an attached low-friction mode of eg5.," Curr. Biol., vol. 14, pp. R411-R412, Jun 2004.

[42] W. R. Schief, R. H. Clark, A. H. Crevenna, and J. Howard, "Inhibition of kinesin motility by adp and phosphate supports a hand-over-hand mechanism.," Proc. Natl. Acad. Sci. U. S. A., vol. 101, pp. 1183-1188, Feb 2004.

[43] B. E. Clancy, W. M. Behnke-Parks, J. O. L. Andreasson, S. S. Rosenfeld, and S. M. Block, "A universal pathway for kinesin stepping.," Nature Structural $\&$ Molecular Biology, vol. 18, pp. 1020-1027, Sep 2011.

[44] E. M. Dagenbach and S. A. Endow, "A new kinesin tree.," J. Cell Sci., vol. 117, pp. 3-7, Jan 2004.

[45] J. Roostalu, C. Hentrich, P. Bieling, I. A. Telley, E. Schiebel, and T. Surrey, "Directional switching of the kinesin cin8 through motor coupling.," Science, vol. 332, pp. 94-99, Apr 2011.

[46] A. Gerson-Gurwitz, C. Thiede, N. Movshovich, V. Fridman, M. Podolskaya, T. Danieli, S. Lakämper, D. R. Klopfenstein, C. F. Schmidt, and L. Gheber, "Directionality of individual kinesin-5 $\operatorname{cin} 8$ motors is modulated by loop 8, ionic strength and microtubule geometry.," EMBO J., vol. 30, pp. 4942-4954, Dec 2011.

[47] C. Thiede, V. Fridman, A. Gerson-Gurwitz, L. Gheber, and C. F. Schmidt, "Regulation of bi-directional movement of single kinesin-5 cin 8 molecules.," Bioarchitecture, vol. 2, pp. 70-74, Feb 2012.

[48] V. Fridman, A. Gerson-Gurwitz, O. Shapira, N. Movshovich, S. Lakämper, C. F. Schmidt, and L. Gheber, "Kinesin-5 kip1 is a bi-directional motor that stabilizes microtubules and tracks their plus-ends in vivo.," J Cell Sci, vol. 126, pp. 4147-4159, Sep 2013.

[49] A. Blangy, H. A. Lane, P. d'Hérin, M. Harper, M. Kress, and E. A. Nigg, "Phosphorylation by p34cdc2 regulates spindle association of human eg5, a kinesin-related motor essential for bipolar spindle formation in vivo.," Cell, vol. 83, pp. 1159-1169, Dec 1995.

[50] N. R. Morris, "Mitotic mutants of aspergillus nidulans.," Genet Res, vol. 26, pp. 237-254, Dec 1975. 
[51] A. P. Enos and N. R. Morris, "Mutation of a gene that encodes a kinesin-like protein blocks nuclear division in a. nidulans.," Cell, vol. 60, pp. 1019-1027, Mar 1990.

[52] I. Hagan and M. Yanagida, "Novel potential mitotic motor protein encoded by the fission yeast cut7+ gene.," Nature, vol. 347, pp. 563-566, Oct 1990.

[53] R. L. Guellec, J. Paris, A. Couturier, C. Roghi, and M. Philippe, "Cloning by differential screening of a xenopus cdna that encodes a kinesin-related protein.," Mol. Cell. Biol., vol. 11, pp. 3395-3398, Jun 1991.

[54] M. A. Hoyt, L. He, K. K. Loo, and W. S. Saunders, "Two saccharomyces cerevisiae kinesin-related gene products required for mitotic spindle assembly.," J. Cell Biol., vol. 118, pp. 109-120, Jul 1992.

[55] M. M. Heck, A. Pereira, P. Pesavento, Y. Yannoni, A. C. Spradling, and L. S. Goldstein, "The kinesin-like protein klp61f is essential for mitosis in drosophila.," J. Cell Biol., vol. 123, pp. 665-679, Nov 1993.

[56] S. M. J. L. van den Wildenberg, L. Tao, L. C. Kapitein, C. F. Schmidt, J. M. Scholey, and E. J. G. Peterman, "The homotetrameric kinesin-5 klp61f preferentially crosslinks microtubules into antiparallel orientations.," Curr. Biol., vol. 18, pp. 1860-1864, Dec 2008.

[57] D. J. Sharp, G. C. Rogers, and J. M. Scholey, "Microtubule motors in mitosis.," Nature, vol. 407, pp. 41-47, Sep 2000.

[58] T. J. Mitchison and E. D. Salmon, "Mitosis: a history of division.," Nat. Cell Biol., vol. 3, pp. E17-E21, Jan 2001.

[59] C. Thiede, Single-molecule experiments with mitotic motor proteins. $\mathrm{PhD}$ thesis, Georg-August-University School of Science (GAUSS), 2012.

[60] T. U. Mayer, T. M. Kapoor, S. J. Haggarty, R. W. King, S. L. Schreiber, and T. J. Mitchison, "Small molecule inhibitor of mitotic spindle bipolarity identified in a phenotype-based screen.," Science, vol. 286, pp. 971-974, Oct 1999.

[61] B. H. Kwok, L. C. Kapitein, J. H. Kim, E. J. G. Peterman, C. F. Schmidt, and T. M. Kapoor, "Allosteric inhibition of kinesin-5 modulates its processive directional motility.," Nat. Chem. Biol., vol. 2, pp. 480-485, Sep 2006.

[62] L. C. Kapitein, B. H. Kwok, J. S. Weinger, C. F. Schmidt, T. M. Kapoor, and E. J. G. Peterman, "Microtubule cross-linking triggers the directional motility of kinesin-5.," J. Cell Biol., vol. 182, pp. 421-428, Aug 2008.

[63] L. C. Kapitein, M. E. Janson, S. M. J. L. van den Wildenberg, C. C. Hoogenraad, C. F. Schmidt, and E. J. G. Peterman, "Microtubule-driven multimerization recruits ase1p onto overlapping microtubules.," Curr. Biol., vol. 18, pp. 1713-1717, Nov 2008.

[64] D. D. Hackney, "Highly processive microtubule-stimulated atp hydrolysis by dimeric kinesin head domains.," Nature, vol. 377, pp. 448-450, Oct 1995. 
[65] H. Y. K. Kaan, D. D. Hackney, and F. Kozielski, "The structure of the kinesin-1 motor-tail complex reveals the mechanism of autoinhibition.," Science, vol. 333, pp. 883-885, Aug 2011.

[66] S. Acar, D. B. Carlson, M. S. Budamagunta, V. Yarov-Yarovoy, J. J. Correia, M. R. Niñonuevo, W. Jia, L. Tao, J. A. Leary, J. C. Voss, J. E. Evans, and J. M. Scholey, "The bipolar assembly domain of the mitotic motor kinesin-5.," Nat. Commun., vol. 4, p. 1343, Jan 2013.

[67] J. S. Weinger, M. Qiu, G. Yang, and T. M. Kapoor, "A nonmotor microtubule binding site in kinesin-5 is required for filament crosslinking and sliding.," Curr. Biol., vol. 21, pp. 154-160, Jan 2011.

[68] S. Lakämper, C. Thiede, A. Düselder, S. Reiter, M. J. Korneev, L. C. Kapitein, E. J. G. Peterman, and C. F. Schmidt, "The effect of monastrol on the processive motility of a dimeric kinesin-5 head/kinesin-1 stalk chimera.," J. Mol. Biol., vol. 399, pp. 1-8, May 2010.

[69] C. Thiede, S. Lakämper, A. D. Wessel, S. Kramer, and C. F. Schmidt, "A chimeric kinesin-1 head/kinesin-5 tail motor switches between diffusive and processive motility.," Biophys. J., vol. 104, pp. 432-441, Jan 2013.

[70] A. Ashkin, "Acceleration and trapping of particles by radiation pressure," Phys. Rev. Lett., vol. 24, pp. 156-159, 1970.

[71] A. Ashkin and J. M. Dziedzic, "Optical levitation by radiation pressure," Appl. Phys. Lett., vol. 19, pp. 283-285, 1971.

[72] A. Ashkin, J. M. Dziedzic, J. E. Bjorkholm, and S. Chu, "Observation of a single-beam gradient force optical trap for dielectric particles," Opt. Lett., vol. 11, pp. 288-290, 1986.

[73] A. Ashkin, "Forces of a single-beam gradient laser trap on a dielectric sphere in the ray optics regime.," Biophys J, vol. 61, pp. 569-582, Feb 1992.

[74] F. Gittes and C. F. Schmidt, "Interference model for back-focal-plane displacement detection in optical tweezers.," Opt. Lett., vol. 23, pp. 7-9, Jan 1998.

[75] A. Pralle, M. Prummer, E. L. Florin, E. H. Stelzer, and J. K. Hörber, "Threedimensional high-resolution particle tracking for optical tweezers by forward scattered light.," Microsc Res Tech, vol. 44, pp. 378-386, Mar 1999.

[76] F. Gittes and C. F. Schmidt, "Signals and noise in micromechanical measurements.," Methods Cell Biol., vol. 55, pp. 129-156, 1998.

[77] K. Svoboda and S. M. Block, "Force and velocity measured for single kinesin molecules.," Cell, vol. 77, pp. 773-784, Jun 1994.

[78] S. Lakämper and E. Meyhöfer, "The e-hook of tubulin interacts with kinesin's head to increase processivity and speed.," Biophys. J., vol. 89, pp. 3223-3234, Nov 2005. 
[79] A. Düselder, C. Thiede, C. F. Schmidt, and S. Lakämper, "Neck-linker length dependence of processive kinesin-5 motility.," J. Mol. Biol., vol. 423, pp. 159168 , Oct 2012 .

[80] T. M. Kapoor and T. J. Mitchison, "Eg5 is static in bipolar spindles relative to tubulin: evidence for a static spindle matrix.," J. Cell Biol., vol. 154, pp. 1125-1133, Sep 2001.

[81] P. Hovland, J. Flick, M. Johnston, and R. A. Sclafani, "Galactose as a gratuitous inducer of gal gene expression in yeasts growing on glucose.," Gene, vol. 83, pp. 57-64, Nov 1989.

[82] G. J. Brouhard, J. H. Stear, T. L. Noetzel, J. Al-Bassam, K. Kinoshita, S. C. Harrison, J. Howard, and A. A. Hyman, "Xmap215 is a processive microtubule polymerase.," Cell, vol. 132, pp. 79-88, Jan 2008.

[83] D. R. Klopfenstein, M. Tomishige, N. Stuurman, and R. D. Vale, "Role of phosphatidylinositol $(4,5)$ bisphosphate organization in membrane transport by the unc104 kinesin motor.," Cell, vol. 109, pp. 347-358, May 2002.

[84] M. Tomishige, D. R. Klopfenstein, and R. D. Vale, "Conversion of unc104/kif1a kinesin into a processive motor after dimerization.," Science, vol. 297, pp. 2263-2267, Sep 2002.

[85] H. Yardimci, M. van Duffelen, Y. Mao, S. S. Rosenfeld, and P. R. Selvin, "The mitotic kinesin cenp-e is a processive transport motor.," Proc. Natl. Acad. Sci. U. S. A., vol. 105, pp. 6016-6021, Apr 2008.

[86] V. Varga, J. Helenius, K. Tanaka, A. A. Hyman, T. U. Tanaka, and J. Howard, "Yeast kinesin-8 depolymerizes microtubules in a length-dependent manner.," Nat. Cell Biol., vol. 8, pp. 957-962, Sep 2006.

[87] V. Hariharan and W. O. Hancock, "Insights into the mechanical properties of the kinesin neck linker domain from sequence analysis and molecular dynamics simulations.," Cell Mol Bioeng, vol. 2, pp. 177-189, Jun 2009.

[88] M. T. Valentine, P. M. Fordyce, T. C. Krzysiak, S. P. Gilbert, and S. M. Block, "Individual dimers of the mitotic kinesin motor eg5 step processively and support substantial loads in vitro.," Nat. Cell Biol., vol. 8, pp. 470-476, May 2006.

[89] T. C. Krzysiak and S. P. Gilbert, "Dimeric eg5 maintains processivity through alternating-site catalysis with rate-limiting atp hydrolysis.," J. Biol. Chem., vol. 281, pp. 39444-39454, Dec 2006.

[90] A. Yildiz, M. Tomishige, A. Gennerich, and R. D. Vale, "Intramolecular strain coordinates kinesin stepping behavior along microtubules.," Cell, vol. 134, pp. 1030-1041, Sep 2008.

[91] S. Shastry and W. O. Hancock, "Neck linker length determines the degree of processivity in kinesin-1 and kinesin-2 motors.," Curr. Biol., vol. 20, pp. 939943, May 2010. 
[92] P. S. Arora, A. Z. Ansari, T. P. Best, M. Ptashne, and P. B. Dervan, "Design of artificial transcriptional activators with rigid poly-l-proline linkers.," J. Am. Chem. Soc., vol. 124, pp. 13067-13071, Nov 2002.

[93] A. Lupas, M. V. Dyke, and J. Stock, "Predicting coiled coils from protein sequences.," Science, vol. 252, pp. 1162-1164, May 1991.

[94] J. W. J. Kerssemakers, E. L. Munteanu, L. Laan, T. L. Noetzel, M. E. Janson, and M. Dogterom, "Assembly dynamics of microtubules at molecular resolution.," Nature, vol. 442, pp. 709-712, Aug 2006.

[95] J. Howard, Mechanics of Motor Proteins and the Cytoskeleton. Sinauer Associates, 2001.

[96] J. Turner, R. Anderson, J. Guo, C. Beraud, R. Fletterick, and R. Sakowicz, "Crystal structure of the mitotic spindle kinesin eg5 reveals a novel conformation of the neck-linker.," J. Biol. Chem., vol. 276, pp. 25496-25502, Jul 2001 .

[97] S. S. Rosenfeld, J. Xing, G. M. Jefferson, and P. H. King, "Docking and rolling, a model of how the mitotic motor eg5 works.," J. Biol. Chem., vol. 280, pp. 35684-35695, Oct 2005.

[98] J. D. Tytell and P. K. Sorger, "Analysis of kinesin motor function at budding yeast kinetochores.," J Cell Biol, vol. 172, pp. 861-874, Mar 2006.

[99] M. K. Chee and S. B. Haase, "B-cyclin/cdks regulate mitotic spindle assembly by phosphorylating kinesins-5 in budding yeast.," PLoS Genet, vol. 6, p. e1000935, May 2010.

[100] L. van Hove, "Correlations in space and time and born approximation scattering in systems of interacting particles," Physical Review, vol. 95, pp. 249-262, 1954.

[101] T. Toyota, D. A. Head, C. F. Schmidt, and D. Mizuno, "Non-gaussian athermal fluctuations in active gels," Soft Matter, vol. 7, pp. 3234-3239, 2011.

[102] W. K. Kegel and A. van Blaaderen, "Direct observation of dynamical heterogeneities in colloidal hard-sphere suspensions," Science, vol. 287, pp. 290-293, Jan 2000 .

[103] M. Braun, Z. Lansky, G. Fink, F. Ruhnow, S. Diez, and M. E. Janson, “Adaptive braking by ase1 prevents overlapping microtubules from sliding completely apart.," Nat. Cell Biol., vol. 13, pp. 1259-1264, Oct 2011.

[104] B. Mickey and J. Howard, "Rigidity of microtubules is increased by stabilizing agents.," J Cell Biol, vol. 130, pp. 909-917, Aug 1995.

[105] T. Wittmann, A. Hyman, and A. Desai, "The spindle: a dynamic assembly of microtubules and motors.," Nat Cell Biol, vol. 3, pp. E28-E34, Jan 2001. 
[106] D. M. Gordon and D. M. Roof, "The kinesin-related protein kip1p of saccharomyces cerevisiae is bipolar.," J. Biol. Chem., vol. 274, pp. 28779-28786, Oct 1999.

[107] E. R. Hildebrandt and M. A. Hoyt, "Mitotic motors in saccharomyces cerevisiae.," Biochim Biophys Acta, vol. 1496, pp. 99-116, Mar 2000.

[108] D. A. Compton, "Spindle assembly in animal cells.," Annu Rev Biochem, vol. 69 , pp. $95-114,2000$.

[109] T. Yanagida, M. Iwaki, and Y. Ishii, "Single molecule measurements and molecular motors.," Philos. Trans. R. Soc. Lond. B Biol. Sci., vol. 363, pp. 2123-2134, Jun 2008.

[110] C. Shingyoji and K. Takahashi, "Cyclical bending movements induced locally by successive iontophoretic application of atp to an elastase-treated flagellar axoneme.," J Cell Sci, vol. 108 ( Pt 4), pp. 1359-1369, Apr 1995.

[111] C. Shingyoji, H. Higuchi, M. Yoshimura, E. Katayama, and T. Yanagida, "Dynein arms are oscillating force generators.," Nature, vol. 393, pp. 711-714, Jun 1998.

[112] I. Ibañez-Tallon, N. Heintz, and H. Omran, "To beat or not to beat: roles of cilia in development and disease.," Hum Mol Genet, vol. 12 Spec No 1, pp. R27-R35, Apr 2003.

[113] M. E. Porter and W. S. Sale, "The $9+2$ axoneme anchors multiple inner arm dyneins and a network of kinases and phosphatases that control motility.," $J$ Cell Biol, vol. 151, pp. F37-F42, Nov 2000.

[114] S. M. Block, L. S. Goldstein, and B. J. Schnapp, "Bead movement by single kinesin molecules studied with optical tweezers.," Nature, vol. 348, pp. 348352, Nov 1990.

[115] C. M. Coppin, J. T. Finer, J. A. Spudich, and R. D. Vale, "Detection of sub-8nm movements of kinesin by high-resolution optical-trap microscopy.," Proc. Natl. Acad. Sci. U. S. A., vol. 93, pp. 1913-1917, Mar 1996.

[116] G. B. Witman, "Isolation of chlamydomonas flagella and flagellar axonemes.," Methods Enzymol., vol. 134, pp. 280-290, 1986.

[117] J. Cahu and T. Surrey, "Motile microtubule crosslinkers require distinct dynamic properties for correct functioning during spindle organization in xenopus egg extract.," J. Cell Sci., vol. 122, pp. 1295-1300, May 2009.

[118] D. S. Gorman and R. P. Levine, "Cytochrome $f$ and plastocyanin: their sequence in the photosynthetic electron transport chain of chlamydomonas reinhardi.," Proc. Natl. Acad. Sci. U. S. A., vol. 54, pp. 1665-1669, Dec 1965.

[119] S. Hutner, L. Provasoli, A. Schatz, and C. Haskins, "Some approaches to the study of the role of metals in the metabolism of microorganisms," Proc. Am. Philos. Soc., vol. 94, pp. 152-170, 1950. 
[120] S. S. Merchant, M. D. Allen, J. Kropat, J. L. Moseley, J. C. Long, S. Tottey, and A. M. Terauchi, "Between a rock and a hard place: trace element nutrition in chlamydomonas.," Biochim Biophys Acta, vol. 1763, pp. 578-594, Jul 2006. 


\section{Acknowledgements}

First and foremost, I would like to thank my supervisor Prof. Christoph Schmidt for turning my interest to biophysics, especially molecular motors, and providing me with many ideas for this thesis. I would also like to thank my thesis committee members, Prof. Sarah Köster - special thanks for acting as the second referee of this thesis - and Oskar Hallatschek, for their support and helpful advice.

In our Institute, one group of people is absolutely essential: our T.A.s! Not only do they relentlessly work so we can pursue our experiments but they are also never short on encouraging things to say! For this, I'd like to thank Ulrike Schulz and Steffi Kramer. I'd especially like to thank Kerstin von Roden for never giving up on either me or "my" proteins and Charlotte Willms for countless hours in which the solution for every problem seemed to be within our grasp!

My biggest thanks go to my colleagues from the Third Institute of Physics Biophysics. You are the best. Without you people, I would never have gotten so far and I am utterly grateful that I had the opportunity to work side by side with you. The support for each other and the feeling that we are more than just colleagues is what kept me going!!! With this I'd like to thank Christopher Battle, Iwan Schaap, Florian Rehfeldt, Dieter Klopfenstein, Carina Wollnik, Ulrich Fromme, Alok Wessel, Nikta Fakhri, Achintya Prahlad, Theresa Kaufeld, Eugenia Butkevich, and Alice Wiesbaum.

My special tanks go to: Christina Thiede for having my back, and countless hours spent together in- and outside of the lab; "Minu" Meenakshi "Kitten" Prabhune for being my (often ignored) voice of reason, for sheering me up untold times and for still(!!) listening; Marcel Bremerich for teaching me everything about optical traps and, in his words, sharing the same exquisite taste of music; Paula Sanchez Baeza for Pisco Sour, teaching me about myself, and messing with my head; and Galina "Goose" Kudryasheva for raising the level of madness and being the best victim imaginable.

I'd also like to thank Florian Schlosser for being The Flo; Miquel Banchs Piqué for great times and "tschan tschan tschaaan"; and Adam Walker for talking nerdy to me and for completing the monumental task of proofreading this work.

In addition to the people from the Institute, I'd like to thank my outside-world friends for distracting me from my work, for providing me with some of the best memories of my life, and for listening, understanding and encouraging!!! My life would be pretty dull without the lot of you and I had certainly way less stories to 
tell!! Therefore, I'd like to especially thank Florian Schlosser, Karina Zimmermann, Stefan Rudas, Tobias Reusch, Friederike Prüser, Katharina Burmeister, HaJo Helms, Max Gulde, Mareike Stamm, Robert Gröbel, Brita Rohrbeck, Meike Schweisfurth, Stefan Küchemann, and Anke Bludau. I owe you all so, so much!!!

Furthermore, I want to thank my parents, Monika and Wolfgang, and my brothers, Timo and Henning, for their constant encouragement and support. I am proud to be part of this family!

In particular, I want to thank Marie Schmidt for being there for me, for always encouraging me, for forcing me to do sports, and for, if needed, kicking my butt!

\section{Thank you!}




\section{Curriculum Vitae}

\section{Personal data}

Name:

Address:

E-Mail:

Date of birth:

Nationality:

11/2009 - Present

$11 / 2009$

$10 / 2004-10 / 2009$
André Düselder

Schillerstraße 38

37083 Göttingen

Germany

Andre.Dueselder@physik3.gwdg.de

$11 / 27 / 1983$

German

\section{Education}

\section{PhD studies in Physics}

Georg-August University Göttingen, Germany

Third Institute of Physics - Biophysics

PhD thesis: "Single-molecule measurements of Kinesin motor proteins"

Supervisor: Prof. Christoph F. Schmidt

Accepted at the Göttingen Graduate School for Neurosciences, Biophysics, and Molecular Biosciences

within the doctoral program "Physics of Biological and Complex Systems"

Diploma in Physics (equivalent to M.Sc.)

Georg-August University Göttingen, Germany

Third Institute of Physics - Biophysics

Diploma thesis: "Einzelmolekülmessungen des Motorproteins Eg5Kin mit einer optischen Falle"

Supervisor: Prof. Christoph F. Schmidt

08/2003 - 05/2004 Civil service

Spastikerhilfe Leer e.V., Leer, Germany

08/1996 - 06/2003 Abitur

Ubbo-Emmius-Gymnasium, Leer, Germany 


\section{Publications}

J. Mol. Biol. A. Düselder, C. Thiede, C. F. Schmidt, and S. Lakämper, "Neck-linker length dependence of processive kinesin-5 motility.", vol. 423, pp. 159-168, Oct 2012.

in preparation

A. Düselder, C. Thiede, A. Wiesbaum, V. Fridman, D. Klopfenstein, L. Gheber, and C. F. Schmidt, "Directionality of Single Kinesin-5 Cin8 Molecules is Mediated by the Tail Domains"

October 30, 2013 